A constructive study of the module structure of rings of partial differential operators

A. Quadrat, D. Robertz

RESEARCH REPORT

$\mathbf{N}^{\circ} 8225$

February 2013

Project-Team Disco 



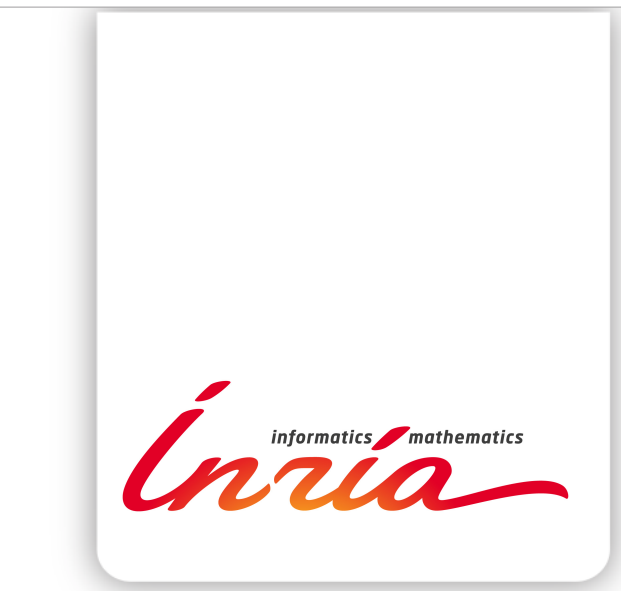

\title{
A constructive study of the module structure of rings of partial differential operators
}

\author{
A. Quadrat*, D. Robertz ${ }^{\dagger}$ \\ Project-Team Disco \\ Research Report n 8225 - February 2013 - 124 pages \\ * Inria Saclay-Île-de-France, DISCO project, Supélec, L2S, 3 rue Joliot Curie, 91192 Gif-sur-Yvette, France. \\ Email: alban.quadrat@inria.fr \\ $\dagger$ Lehrstuhl B für Mathematik, RWTH Aachen University, Templergraben 64, 52056 Aachen, Germany. \\ Email: daniel@momo.math.rwth-aachen.de.
}

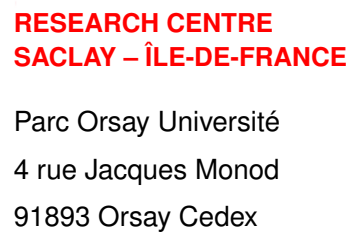


Abstract: The purpose of this paper is to develop constructive versions of Stafford's theorems on the module structure of Weyl algebras $A_{n}(k)$ (i.e., the rings of partial differential operators with polynomial coefficients) over a base field $k$ of characteristic zero. More generally, based on results of Stafford and Coutinho-Holland, we develop constructive versions of Stafford's theorems for very simple domains $D$. The algorithmization is based on the fact that certain inhomogeneous quadratic equations admit solutions in a very simple domain. We show how to explicitly compute a unimodular element of a finitely generated left $D$-module of rank at least two. This result is used to constructively decompose any finitely generated left $D$-module into a direct sum of a free left $D$-module and a left $D$-module of rank at most one. If the latter is torsion-free, then we explicitly show that it is isomorphic to a left ideal of $D$ which can be generated by two elements. Then, we give an algorithm which reduces the number of generators of a finitely presented left $D$-module with module of relations of rank at least two to two. In particular, any finitely generated torsion left $D$-module can be generated by two elements and is the homomorphic image of a projective ideal whose construction is explicitly given. Moreover, a non-torsion but non-free left $D$-module of rank $r$ can be generated by $r+1$ elements but no fewer. These results are implemented in the STAFFORD package for $D=A_{n}(k)$ and their system-theoretical interpretations are given within a $D$-module approach. Finally, we prove that the above results also hold for the ring of ordinary differential operators with either formal power series or locally convergent power series coefficients and, using a result of Caro-Levcovitz, also for the ring of partial differential operators with coefficients in the field of fractions of the ring of formal power series or of the ring of locally convergent power series.

Key-words: Weyl algebras, Stafford's theorems, linear systems of partial differential equations, $D$-modules, mathematical systems theory, constructive algebra, symbolic computation. 


\section{Une étude constructive de la structure des modules sur des anneaux d'opérateurs différentiels}

Résumé : Ce papier a pour but de développer des versions constructives des théorèmes de Stafford qui étudient la structure des modules sur les algèbres de Weyl $A_{n}(k)$ (c'est-à-dire, sur les anneaux d'opérateurs différentiels à coefficients polynomiaux), où $k$ est un corps de caractéristique zéro. Plus généralement, en utilisant des idées de Stafford et Coutinho-Holland, nous développons des versions constructives des théorèmes de Stafford pour des anneaux fortement simples $D$. Cette algorithmisation est basée sur le fait que certaines équations quadratiques inhomogènes admettent des solutions dans un anneau fortement simple. Nous montrons comment obtenir de manière explicite un élément unimodulaire d'un $D$-module à gauche de type fini de rang au moins 2. Ce résultat est utilisé pour décomposer tout $D$ module à gauche de type fini en une somme directe d'un $D$-module libre à gauche et d'un $D$-module à gauche de rang au plus 1 . Si ce dernier est sans-torsion, nous montrons alors de manière explicite qu'il est isomorphe à un idéal à gauche pouvant être engendré par deux éléments. Nous donnons alors un algorithme qui réduit à deux le nombre de générateurs d'un $D$-module à gauche de présentation finie possédant un module de relations de rang au moins 2. En particulier, tout $D$-module à gauche de type fini peut être engendré par deux éléments et il est l'image d'un idéal projectif dont la construction est explicitement donnée. De plus, un $D$-module à gauche de rang $r$, qui n'est ni de torsion, ni libre, peut être engendré par

$r+1$ éléments et pas moins. Ces résultats sont implantés dans le package STAFForD pour $D=A_{n}(k)$ et l'interprétation en terme de théorie mathématique des systèmes donnée est basée sur l'approche des $D$-modules. Finalement, nous montrons que ces résultats sont aussi valables pour les anneaux d'opérateurs différentiels ordinaires à coefficients dans l'anneau des séries formelles ou l'anneau des séries localement convergentes et, grâce à un résultat de Caro et Levcovitz, pour les anneaux d'opérateurs aux dérivées partielles à coefficients dans le corps de fractions de l'anneau des séries formelles ou celui des séries localement convergentes.

Mots-clés : Algèbres de Weyl, systèmes linéaires d'équations aux dérivées partielles, $D$ modules, théorie mathématique des systèmes, algèbre constructive, calcul formel. 


\section{Contents}

1 Introduction $\quad 5$

2 Algebraic analysis $\quad 8$

3 Unimodular elements and very simple domains $\quad 11$

3.1 Unimodular elements . . . . . . . . . . . . . . . . . . . . . . . . 11

3.2 Very simple domains . . . . . . . . . . . . . . . . . . . . . 14

3.3 Computation of unimodular elements . . . . . . . . . . . . . . . 17

4 Stafford's reduction $\quad 31$

$5 \quad$ Efficient generation of finitely generated modules $\quad 37$

6 Cancellation theorem $\quad 46$

6.1 Main result . . . . . . . . . . . . . . . . . . 46

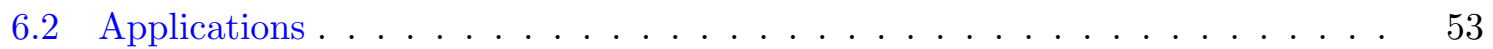

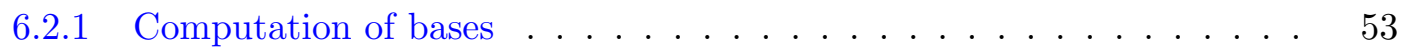

6.2.2 Rank reduction compatible with isomorphism . . . . . . . . . . 56

6.2 .3 Auslander transposes . . . . . . . . . . . . . 56

$\begin{array}{lll}7 & \text { Conclusion } & 60\end{array}$

8 Appendix: New commands of the STAFFORD package $\quad 61$

8.1 Strong generation, unimodular elements, and Serre's splitting-off theorem . . 62

8.2 Stafford's reduction . . . . . . . . . . . . . . . . 81

8.3 Computation of bases of finitely generated free modules . . . . . . . . . . 93

8.4 Cancellation theorem . . . . . . . . . . . . . . . . . . 101 


\section{Introduction}

In his seminal paper [42], Stafford precisely described the module structure of the Weyl algebra $A_{n}(k)$ over a field $k$ of characteristic zero, namely, of the ring of partial differential (PD) operators with coefficients that are polynomials over $k$. In particular, he proved his famous result asserting that every left/right ideal of $A_{n}(k)$ can be generated by two elements. Constructive proofs of this result were studied in [21, 25] and implemented in Macaulay2 ([26]) and in Maple ([36]). A consequence of Stafford's theorem is that every finitely generated projective (or, equivalently, stably free) left/right module over $A_{n}(k)$ of rank at least two is free ([42]). This result was made constructive in [20] by following Stafford's original proof and in [36] by developing a more general one based on the concept of stable rank (Stafford's theorem asserting that the stable rank of $A_{n}(k)$ is two). This last algorithm was implemented in the STAFFORD package ([36]) and used in mathematical systems theory ([36]).

Based on extensions of Stafford's results obtained in [18], we proved in [37] that the same results as the ones developed in [42] are valid for the ring of ordinary differential (OD) operators with coefficients in the ring of formal power series or in the ring of real or complex convergent power series. This result was applied to mathematical systems theory (e.g., reduction and decomposition problems, Serre's reduction, controllability, observability, Monge problem, computation of flat outputs and injective parametrizations of differentially flat systems, blowing-up of singularities). For more details, see [13, 15, 37].

Stafford's theorem has also been extended in [9] to the ring $\widehat{\mathcal{D}}_{n}(k)$ (resp., $\mathcal{D}_{n}(k)$ ) of PD operators with coefficients in the field of fractions of the ring of formal power series $\widehat{\mathcal{A}}=k \llbracket x_{1}, \ldots, x_{n} \rrbracket$ (resp., of the ring of locally convergent power series $\mathcal{A}=k\left\{x_{1}, \ldots, x_{n}\right\}$ ). As explained in $[36,37]$, projective modules of rank at least two over these algebras are free.

More results on the module structure of the Weyl algebra $D=A_{n}(k)$, obtained by Stafford in [42], have not been made constructive yet, and in particular the following ones (where every module is assumed or claimed to be finitely generated):

1. Every left $D$-module $M$ can be decomposed as the direct sum of a free left $D$-module $D^{1 \times r}$ for some $r \in \mathbb{Z}_{\geq 0}$ and a left $D$-module $M^{\prime}$ of rank at most 1 , i.e., $M \cong D^{1 \times r} \oplus M^{\prime}$, where $\operatorname{rank}_{D}\left(M^{\prime}\right) \leq 1$.

2. Every torsion-free left $D$-module $M$ can be decomposed as the direct sum of a free left $D$-module $D^{1 \times r}$ for some $r \in \mathbb{Z}_{\geq 0}$ and a left ideal $I$ of $D$ generated by two elements, i.e., $M \cong D^{1 \times r} \oplus I$, where $I=D d_{1}+D d_{2}$ for certain $d_{1}, d_{2} \in D$.

3. Every torsion left $D$-module $T$ is the homomorphic image of a projective left ideal of $D$, and thus can be generated by two elements, i.e., $T \cong I / J$, where $I$ is a projective left ideal of $D$ and $J$ a left $D$-submodule of $I$ (which can also be generated by two elements).

$\mathrm{RR} \mathrm{n}^{\circ} 8225$ 
4. Every non-torsion left $D$-module of rank $r$ is either free or can be generated by $r+1$ elements but no fewer.

5. If $\operatorname{rank}_{D}(M) \geq 2$ and $M \oplus D \cong N \oplus D$, then $M \cong N$.

Similar results hold for finitely generated right $D$-modules ([42]).

The first (resp., last) point above can be traced back to Serre's splitting-off theorem ([39]) (resp., Bass' cancellation theorem ([5])), not requiring, however, any projectivity condition on $M$. These results are well-known in commutative algebra and they have been studied in $[17,27]$ (see also the references therein) within a constructive commutative algebra approach.

Versions of these results were studied in $[40,41]$ for noncommutative (simple) noetherian rings and in [18] for very simple domains. A very simple ring $D$ is a prime ring $D$ (namely, $d_{1} D d_{2} \neq 0$ for all $d_{1}, d_{2} \in D \backslash\{0\}$ ) which is noetherian and satisfies

$$
\forall a, b, c \in D, \forall d \in D \backslash\{0\}, \exists u, v \in D: D a+D b+D c=D(a+d u c)+D(b+d v c),
$$

where $D s=\{r s \mid r \in D\}$ denotes the principal left ideal of $D$ generated by $s \in D$. For more details, see [18]. If $D$ is a very simple domain, then one can easily show that for fixed $d_{1}, d_{2} \in D \backslash\{0\}$, the following inhomogeneous quadratic equation

$$
y_{1} d_{1} z_{1}+y_{2} d_{2} z_{2}=1
$$

admit solutions $\left(\begin{array}{llll}y_{1} & y_{2} & z_{1} & z_{2}\end{array}\right)^{T} \in D^{4}[18,42]$. The Weyl algebra $A_{n}(k)$, where $k$ is a field of characteristic zero, is a prototypical example of a very simple domain. The solvability of the above equation plays a fundamental role in certain problems studied in module theory (e.g., existence of unimodular elements, direct decomposition of modules). In particular, Stafford's theorems, stated above for $A_{n}(k)$, hold for a very simple domain $D$. More generally, it can easily be seen that the (category of) finitely generated modules over a very simple domain possess(es) some features that bear some resemblance with (the category of) finitely generated vector spaces over a division ring (e.g., a field).

The purpose of this paper is to study the above results for very simple domains from a constructive viewpoint, therefore completing our understanding of Stafford's results for $A_{n}(k)$ ([42]) in terms of symbolic computation. Using the recent extension of Stafford's main theorem to the rings $\widehat{\mathcal{D}}_{n}(k)$ and $\mathcal{D}_{n}(k)$ obtained in [9], we also generalize Stafford's theorems to the algebras $\widehat{\mathcal{D}}_{n}(k)$ and $\mathcal{D}_{n}(k)$. Finally, the algorithms we developed in the present paper for $A_{n}(\mathbb{Q})$ are implemented in the STAFFORD package ([36]).

From a more general perspective, the constructive approach to algebraic analysis initiated in $[1,3,4,7,11,12,13,14,15,16,31,32,33,34]$ relies on the solvability of inhomogeneous linear systems in the ring under consideration. Within category theory, this idea was axiomatized in [2] and applied, for instance, to sheaf theory. If $D$ is a very simple domain, in this paper, we show that problems classically studied in module theory (e.g., Serre's splitting-off

$\mathrm{RR} \mathrm{n}^{\circ} 8225$ 
theorem, Swan's lemma, Bass' theorem), which are based on the solvability of inhomogeneous quadratic equations (see above), can be studied within the same constructive framework.

One of the main motivations for making Stafford's results constructive is the fact that they have system-theoretic interpretations within the algebraic analysis or D-modules approach ([6, $23,28,29])$ (e.g., efficient generation of the set of autonomous elements ([11, 36]), computation of injective parametrizations ([36]), Serre's reduction $([7,15])$, decomposability of the solution space $([13]))$. These results apply to general linear PD systems which can be determined, overdetermined, or underdetermined depending on which result we consider. For instance, apart from many other results, we prove that a linear system of PDEs defined by a full row rank matrix of PD operators (e.g., most of the classical linear systems of PDEs studied in mathematical physics and engineering science) is equivalent to a linear system defined by two PDEs in two unknown functions (see Example 9). This result can be seen as a generalization for linear systems of PDEs of the well-known cyclic vector theorem in the theory of ODEs (see, e.g., [10] and the references therein). Moreover, these results can be used to study nonlinear PD systems by means of their linearizations around a polynomial/rational/formal power series/locally convergent power series solution (e.g., shallow water waves, Poiseuille flow, flexible thread attached to one point in a vertical equilibrium position).

We point out that the above results are not valid for a ring of PD operators with constant coefficients. But if one allows the use of non-constant coefficients, then we can always embed this ring into a ring of PD operators with polynomial/rational/formal power series/locally convergent power series coefficients, so that the above results hold (see, e.g., Example 5).

The paper is organized as follows. In Section 2, we shortly introduce basic ideas of algebraic analysis, review well-known results of noncommutative algebra, and explain how linear PD systems can be studied by means of module theory. The main results of the paper are presented in Section 3. More precisely, the fundamental role of the unimodular elements of a module is explained in Section 3.1. Section 3.3 shows how to combine Stafford's main theorem with constructive module-theoretic techniques to explicitly compute unimodular elements of (sub)modules. These results are then used in Section 4 to study Stafford's reduction and in Section 5 to examine questions about the efficient generation of modules. In Section 6, we study Stafford's cancellation theorem and its consequences. We conclude and open some perspectives in Section 7. Finally, in an appendix, we demonstrate the new commands of the STAFFORD package on different explicit examples.

Notation. In what follows, $\mathbb{Z}_{\geq 0}$ will denote the set of non-negative integers, $k$ a field of characteristic zero (e.g., $k \in\{\mathbb{Q}, \mathbb{R}, \mathbb{C}\}$ ), D a noetherian domain, namely, a left and right noetherian ring with 1 and without zero-divisors, and $D^{q \times p}$ the set of $q \times p$ matrices with entries in $D$. We shall identify $D^{q}$ and $D^{q \times 1}$ and we write $I_{r}$ for the $r \times r$ identity matrix. Moreover,

$$
\mathrm{GL}_{r}(D)=\left\{U \in D^{r \times r} \mid \exists V \in D^{r \times r}: U V=V U=I_{r}\right\}
$$

$\mathrm{RR} \mathrm{n}^{\circ} 8225$ 
is the general linear group of degree $r$ over $D$ and $\mathrm{U}(D)=\mathrm{GL}_{1}(D)$ the group of units of $D$.

\section{Algebraic analysis}

Using ideas of algebraic analysis ([6, 23, 28, 29]), we can study linear systems over a noetherian domain $D$ by means of module theory and homological algebra. We shortly recall a few results which will be useful in what follows. For more details and results, we refer to [11, 13, 36].

Let $R \in D^{q \times p}$ and $M:=D^{1 \times p} /\left(D^{1 \times q} R\right)$ be the left $D$-module finitely presented by $R$. Let us describe $M$ by means of generators and relations. Let $\left\{f_{j}\right\}_{j=1, \ldots, p}$ be the standard basis of $D^{1 \times p}$, namely, $f_{j}$ is the row vector of length $p$ with 1 at the $j^{\text {th }}$ position and 0 elsewhere. Moreover, let $\pi: D^{1 \times p} \longrightarrow M$ be the left $D$-homomorphism defined by sending $\lambda \in D^{1 \times p}$ to its residue class $\pi(\lambda)$ in $M$. By definition of $M, \pi$ is surjective since every $m \in M$ is the residue class of certain $\lambda$ 's in $D^{1 \times p}$, i.e., $m=\pi(\lambda)=\pi(\lambda+\nu R)$ for all $\nu \in D^{1 \times q}$. If $y_{j}=\pi\left(f_{j}\right)$ for $j=1, \ldots, p$, then, for every $m \in M$, there exists $\lambda=\left(\lambda_{1} \ldots \lambda_{p}\right) \in D^{1 \times p}$ such that

$$
m=\pi(\lambda)=\pi\left(\sum_{j=1}^{p} \lambda_{j} f_{j}\right)=\sum_{j=1}^{p} \lambda_{j} \pi\left(f_{j}\right)=\sum_{j=1}^{p} \lambda_{j} y_{j}
$$

which shows that $\left\{y_{j}\right\}_{j=1, \ldots, p}$ is a family of generators of $M$. Let $R_{i}$ (resp., $R_{\bullet}$ ) denote the $i^{\text {th }}$ row (resp., $j^{\text {th }}$ column) of $R$. The family of generators $\left\{y_{j}\right\}_{j=1, \ldots, p}$ of $M$ satisfies relations

$$
\forall i=1, \ldots, q, \quad \sum_{j=1}^{p} R_{i j} y_{j}=\sum_{j=1}^{p} R_{i j} \pi\left(f_{j}\right)=\pi\left(\sum_{j=1}^{p} R_{i j} f_{j}\right)=\pi\left(R_{i \bullet}\right)=0,
$$

since $R_{i} \bullet D^{1 \times q} R$ for $i=1, \ldots, q$. Now, let $\mathcal{F}$ be a left $D$-module and let

$$
\operatorname{ker}_{\mathcal{F}}(R .):=\left\{\eta \in \mathcal{F}^{p} \mid R \eta=0\right\}
$$

be the linear system defined by $R$ and $\mathcal{F}$. A simple but fundamental remark due to Malgrange ([29]) is that the linear system $\operatorname{ker}_{\mathcal{F}}\left(R\right.$.) is isomorphic to the abelian group $\operatorname{hom}_{D}(M, \mathcal{F})$ of left $D$-homomorphisms from $M$ to $\mathcal{F}$, i.e.,

$$
\operatorname{ker}_{\mathcal{F}}(R .) \cong \operatorname{hom}_{D}(M, \mathcal{F})
$$

as abelian groups, where $\cong$ denotes an isomorphism (e.g., of abelian groups, left/right modules). This isomorphism can easily be described: if $\varphi \in \operatorname{hom}_{D}(M, \mathcal{F}), \eta_{j}=\varphi\left(y_{j}\right)$ for $j=1, \ldots, p$, and $\eta=\left(\eta_{1} \ldots \eta_{p}\right)^{T} \in \mathcal{F}^{p}$, then using (1), $R \eta=0$ since for $i=1, \ldots, q$ :

$$
\sum_{j=1}^{p} R_{i j} \eta_{j}=\sum_{j=1}^{p} R_{i j} \varphi\left(y_{j}\right)=\varphi\left(\sum_{j=1}^{p} R_{i j} y_{j}\right)=\varphi\left(\sum_{j=1}^{p} R_{i j} \pi\left(f_{j}\right)\right)=\varphi\left(\pi\left(R_{i \bullet}\right)\right)=\varphi(0)=0 .
$$

$\mathrm{RR} \mathrm{n}^{\circ} 8225$ 
Moreover, we can show that given $\eta=\left(\eta_{1} \ldots \eta_{p}\right)^{T} \in \operatorname{ker}_{\mathcal{F}}(R$. $)$, the map $\varphi_{\eta}: M \longrightarrow \mathcal{F}$ defined by $\varphi_{\eta}(\pi(\lambda))=\lambda \eta$ for $\lambda \in D^{1 \times p}$ is a well-defined left $D$-homomorphism from $M$ to $\mathcal{F}$, i.e., $\varphi_{\eta} \in \operatorname{hom}_{D}(M, \mathcal{F})$. Finally, the abelian group homomorphism $\chi: \operatorname{ker}_{\mathcal{F}}(R.) \longrightarrow \operatorname{hom}_{D}(M, \mathcal{F})$ defined by $\chi(\eta)=\varphi_{\eta}$ is bijective. For more details, see [11, 13, 36]. Hence, $(2)$ shows that the properties of the linear system $\operatorname{ker}_{\mathcal{F}}\left(R\right.$.) can be studied by means of $\operatorname{hom}_{D}(M, \mathcal{F})$, and thus by means of the left $D$-modules $M$ and $\mathcal{F}$.

If $M^{\prime}, M, M^{\prime \prime}$ are three left/right $D$-modules, $f \in \operatorname{hom}_{D}\left(M^{\prime}, M\right)$, and $g \in \operatorname{hom}_{D}\left(M, M^{\prime \prime}\right)$, then $M^{\prime} \stackrel{f}{\longrightarrow} M \stackrel{g}{\longrightarrow} M^{\prime \prime}$ is called a complex if $\operatorname{im} f \subseteq \operatorname{ker} g$. This complex is said to be exact at $M$ if $\operatorname{ker} g=\operatorname{im} f$. A complex which is exact at any place is called an exact sequence. In particular, the complex $0 \longrightarrow M^{\prime} \stackrel{f}{\longrightarrow} M \stackrel{g}{\longrightarrow} M^{\prime \prime} \longrightarrow 0$ is called a short exact sequence if $f$ is injective, $\operatorname{ker} g=\operatorname{im} f$, and $g$ is surjective. For more details, see [38].

The left $D$-module $M=D^{1 \times p} /\left(D^{1 \times q} R\right)$ yields the following exact sequence

$$
D^{1 \times q} \stackrel{. R}{\longrightarrow} D^{1 \times p} \stackrel{\pi}{\longrightarrow} M \longrightarrow 0
$$

called a presentation of $M$, where $. R: D^{1 \times q} \longrightarrow D^{1 \times p}$ is defined by $(. R)(\mu)=\mu R$ for all $\mu \in D^{1 \times q}$. Since matrices $R_{1}$ and $R_{2}$ representing equivalent linear systems define left $D$ isomorphic modules, $\operatorname{hom}_{D}(M, \mathcal{F})$ is a more intrinsic characterization of the linear system than $\operatorname{ker}_{\mathcal{F}}\left(R\right.$.) (e.g., it does not depend on the particular embedding of $\operatorname{ker}_{\mathcal{F}}\left(R\right.$.) into $\left.\mathcal{F}^{p}\right)$.

Example 1. Let $A$ be a differential ring, namely, $A$ is a commutative ring equipped with commuting derivations $\delta_{i}$ for $i=1, \ldots, n$, namely, maps $\delta_{i}: A \longrightarrow A$ satisfying

$$
\forall a_{1}, a_{2} \in A, \quad \delta_{i}\left(a_{1}+a_{2}\right)=\delta_{i}\left(a_{1}\right)+\delta_{i}\left(a_{2}\right), \quad \delta_{i}\left(a_{1} a_{2}\right)=\delta_{i}\left(a_{1}\right) a_{2}+a_{1} \delta_{i}\left(a_{2}\right),
$$

and $\delta_{i} \circ \delta_{j}=\delta_{j} \circ \delta_{i}$ for all $1 \leq i<j \leq n$. Moreover, let $D=A\left\langle\partial_{1}, \ldots, \partial_{n}\right\rangle$ be the (not necessarily commutative) polynomial ring of $\mathrm{PD}$ operators in $\partial_{1}, \ldots, \partial_{n}$ with coefficients in $A$, namely, every element $d \in D$ is of the form

$$
d=\sum_{0 \leq|\mu| \leq r} a_{\mu} \partial^{\mu}, \quad r \in \mathbb{Z}_{\geq 0}, \quad a_{\mu} \in A, \quad \mu=\left(\mu_{1} \ldots \mu_{n}\right) \in \mathbb{Z}_{\geq 0}^{1 \times n},
$$

where $\partial^{\mu}=\partial_{1}^{\mu_{1}} \ldots \partial_{n}^{\mu_{n}}$ is a monomial in the pairwise commuting indeterminates $\partial_{1}, \ldots, \partial_{n}$, and $\partial_{i} a=a \partial_{i}+\delta_{i}(a)$ for all $a \in A$. If $k$ is a field and $A=k\left[x_{1}, \ldots, x_{n}\right]$ (resp., $k\left(x_{1}, \ldots, x_{n}\right)$ ), then $A\left\langle\partial_{1}, \ldots, \partial_{n}\right\rangle$ is simply denoted by $A_{n}(k)$ (resp., $B_{n}(k)$ ) and is called the polynomial (resp., rational) Weyl algebra. If $R \in D^{q \times p}$ and $\mathcal{F}$ is a left $D$-module (e.g., $\mathcal{F}=A$ ), then the linear PD system $\operatorname{ker}_{\mathcal{F}}(R)=.\left\{\eta \in \mathcal{F}^{p} \mid R \eta=0\right\}$ is intrinsically defined by $\operatorname{hom}_{D}(M, \mathcal{F})$, where $M=D^{1 \times p} /\left(D^{1 \times q} R\right)$ is the left $D$-module finitely presented by $R$.

Within algebraic analysis, the study of the module structure of rings of PD operators plays a fundamental role for the study of linear systems of PD equations. In [11, 13, 33, 34, 36], we 
have initiated the constructive study of module theory and homological algebra over rings of functional operators such as rings of PD operators or Ore algebras.

Let us now recall a few classical definitions of module theory (see, e.g., [30, 38]).

Definition 1. Let $D$ be a noetherian domain and $M$ a finitely generated left $D$-module.

- $M$ is free if there exists $r \in \mathbb{Z}_{\geq 0}$ such that $M \cong D^{1 \times r}$. In this case $r$ is called the rank of $M$.

- $M$ is stably free if there exist $r, s \in \mathbb{Z}_{\geq 0}$ such that $M \oplus D^{1 \times s} \cong D^{1 \times r}$, where $\oplus$ denotes the direct sum of modules.

- $M$ is projective if there exist $r \in \mathbb{Z}_{\geq 0}$ and a left $D$-module $P$ such that $M \oplus P \cong D^{1 \times r}$.

- $M$ is reflexive if the canonical left $D$-homomorphism $\varepsilon: M \longrightarrow \operatorname{hom}_{D}\left(\operatorname{hom}_{D}(M, D), D\right)$ defined by $\varepsilon(m)(f)=f(m)$, for all $m \in M$ and for all $f \in \operatorname{hom}_{D}(M, D)$, is an isomorphism of left $D$-modules.

- $M$ is torsion-free if the torsion left $D$-submodule of $M$ defined by

$$
t(M)=\{m \in M \mid \exists d \in D \backslash\{0\}: d m=0\}
$$

is reduced to 0 , i.e., $t(M)=0$.

- $M$ is torsion if $t(M)=M$.

Similar definitions hold for right $D$-modules.

See $[11,33,36]$ for algorithms which test whether or not a finitely presented left $D$-module $M$ is stably free, projective, reflexive, torsion-free, has torsion elements or is torsion.

Since $D$ is a noetherian domain, $D$ satisfies the left and the right Ore properties (see, e.g., [30]), namely, for all $d_{1}, d_{2} \in D \backslash\{0\}$, there exist $e_{1}, e_{2} \in D \backslash\{0\}$ such that $e_{1} d_{1}=e_{2} d_{2}$ (resp., $d_{1} e_{1}=d_{2} e_{2}$ ). This result yields the existence of the division ring of fractions $Q(D)=$ $S^{-1} D=D S^{-1}$ of $D$, where $S=D \backslash\{0\}$ (see, e.g., [30]). If $M$ is a finitely generated left/right $D$-module, then $Q(D) \otimes_{D} M$ (resp., $M \otimes_{D} Q(D)$ ) is a finitely generated left (resp., right) $Q(D)$-vector space and $\operatorname{rank}_{D}(M):=\operatorname{dim}_{Q(D)}\left(Q(D) \otimes_{D} M\right)=\operatorname{dim}_{Q(D)}\left(M \otimes_{D} Q(D)\right)$.

Theorem 1 ([30, 38, 42]). With the hypotheses of Definition 1, the following results hold:

1. The following implications hold for finitely generated left/right D-modules:

$$
\text { free } \Rightarrow \text { stably free } \Rightarrow \text { projective } \Rightarrow \text { reflexive } \Rightarrow \text { torsion-free. }
$$

2. If $D=A_{1}(k)$, then every finitely generated torsion-free left $D$-module is stably free.

$\mathrm{RR} \mathrm{n}^{\circ} 8225$ 
3. If $D$ is a principal ideal domain, namely, every left ideal and every right ideal of $D$ can be generated by one element, (e.g., $\left.D=B_{1}(k)\right)$, then every finitely generated torsion-free left $D$-module is free.

4. If $D=A_{n}(k)$, then every finitely generated projective left/right $D$-module is stably free and every stably free left $D$-module $M$ with $\operatorname{rank}_{D}(M) \geq 2$ is free (Stafford's theorem).

Constructive proofs of Stafford's theorem (see 4 of Theorem 1) were given in $[20,36]$. Computation of bases of free $A_{n}(\mathbb{Q})$-modules is implemented in the STAFFORD package ([36]).

Finally, let us state a proposition that will be constantly used in what follows.

Proposition 1 ([13]). Let $R \in D^{q \times p}$ and $R^{\prime} \in D^{q^{\prime} \times p}$ satisfy $D^{1 \times q} R \subseteq D^{1 \times q^{\prime}} R^{\prime}$, i.e., are such that $R=R^{\prime \prime} R^{\prime}$ for a certain $R^{\prime \prime} \in D^{q \times q^{\prime}}$. Moreover, let $R_{2}^{\prime} \in D^{r^{\prime} \times q^{\prime}}$ be a matrix such that $\operatorname{ker}_{D}\left(. R^{\prime}\right)=D^{1 \times r^{\prime}} R_{2}^{\prime}$, and let $\pi$ and $\pi^{\prime}$ be respectively the following canonical projections:

$$
\begin{aligned}
\pi: D^{1 \times q^{\prime}} R^{\prime} & \longrightarrow P:=\left(D^{1 \times q^{\prime}} R^{\prime}\right) /\left(D^{1 \times q} R\right), \\
\pi^{\prime}: D^{1 \times q^{\prime}} & \longrightarrow Q:=D^{1 \times q^{\prime}} /\left(D^{1 \times q} R^{\prime \prime}+D^{1 \times r^{\prime}} R_{2}^{\prime}\right) .
\end{aligned}
$$

Then, the left D-homomorphism ı defined by

$$
\begin{aligned}
Q & \stackrel{\iota}{\longrightarrow} P \\
\pi^{\prime}(\lambda) & \longmapsto \pi\left(\lambda R^{\prime}\right),
\end{aligned}
$$

is an isomorphism and its inverse $\iota^{-1}$ is defined by:

$$
\begin{aligned}
P & \stackrel{\iota^{-1}}{\longrightarrow} Q \\
\pi\left(\lambda R^{\prime}\right) & \longmapsto \pi^{\prime}(\lambda) .
\end{aligned}
$$

\section{Unimodular elements and very simple domains}

\subsection{Unimodular elements}

Definition 2. An element $m^{\star}$ of a left $D$-module $M$ is called unimodular if there exists $\varphi \in \operatorname{hom}_{D}(M, D)$ such that $\varphi\left(m^{\star}\right)=1$.

The set of unimodular elements of $M$ is denoted by $\mathrm{U}(M)$. Let us explain the significance of unimodular elements in module theory. If $m^{\star} \in \mathrm{U}(M)$, then there exists $\varphi \in \operatorname{hom}_{D}(M, D)$ such that $\varphi\left(m^{\star}\right)=1$. If $\sigma: D \longrightarrow M$ is the left $D$-homomorphism defined by $\sigma(1)=m^{\star}$, then $(\varphi \circ \sigma)(d)=\varphi\left(d m^{\star}\right)=d$ for all $d \in D$, i.e., $\varphi \circ \sigma=\operatorname{id}_{D}$. In particular, $\varphi$ is surjective and we have the following short exact sequence of left $D$-modules:

$$
0 \longrightarrow \operatorname{ker} \varphi \longrightarrow M \stackrel{\varphi}{\longrightarrow} D \longrightarrow 0 \text {. }
$$

$\mathrm{RR} \mathrm{n}^{\circ} 8225$ 
Since $\varphi \circ \sigma=\operatorname{id}_{D}$, the exact sequence (3) splits (see, e.g., [38]), i.e., $M=\operatorname{ker} \varphi \oplus D m^{\star}$, where $D m^{\star}=D \sigma(1) \cong D$ since $\sigma$ is injective. Hence, the left $D$-module $D m^{\star}$ generated by $m^{\star}$ is a direct summand of $M$. Thus, every unimodular element of $M$ can be used to decompose $M$ into a direct sum. Finally, the splitting of (3) yields the following unique decomposition of elements of $M$

$$
\forall m \in M: \quad m=\left(m-\varphi(m) m^{\star}\right)+\varphi(m) m^{\star},
$$

where $m-\varphi(m) m^{\star} \in \operatorname{ker} \varphi$ and $\varphi(m) m^{\star} \in D m^{\star}$.

If $m$ is a torsion element of $M$, i.e., $d m=0$ for some $d \in D \backslash\{0\}$, and if $f \in \operatorname{hom}_{D}(M, D)$, then $d f(m)=f(d m)=f(0)=0$, which shows that $f(m)=0$ since $D$ is a domain. Hence, a unimodular element of $M$ cannot be a torsion element of $M$.

In the sequel we will frequently use the following characterization of torsion modules.

Lemma 1 ([11, 24]). Let $D$ be a noetherian domain and $M$ a finitely generated left $D$-module. Then, the following statements are equivalent:

1. $M$ is torsion.

2. $\operatorname{hom}_{D}(M, D)=0$.

3. $\operatorname{rank}_{D}(M)=0$.

Let us now study the problem of computing unimodular elements of $M$. Let us consider a finitely presented left $D$-module $M=D^{1 \times p} /\left(D^{1 \times q} R\right)$. Malgrange's remark (see (2)) with $\mathcal{F}=D$ yields $\operatorname{hom}_{D}(M, D) \cong \operatorname{ker}_{D}(R$. . More precisely, we have the following simple lemma.

Lemma 2. Let $M=D^{1 \times p} /\left(D^{1 \times q} R\right)$ and $\pi: D^{1 \times p} \longrightarrow M$ be the canonical projection onto $M$. Then, for every $\varphi \in \operatorname{hom}_{D}(M, D)$, there exists $\mu \in \operatorname{ker}_{D}(R$.), i.e., $R \mu=0$, such that:

$$
\forall \lambda \in D^{1 \times p}, \quad \varphi(\pi(\lambda))=\lambda \mu .
$$

Notation. We shall simply denote the left $D$-homomorphism defined by (5) by $\varphi_{\mu}$.

As previously shown, if $M$ is a torsion left $D$-module, then $\mathrm{U}(M)=\emptyset$, i.e., a torsion left $D$-module $M$ does not contain unimodular elements. Hence, let us suppose that $M$ is not a torsion left $D$-module. Then, $2 \Rightarrow 1$ of Lemma 1 shows that $\operatorname{ker}_{D}\left(R\right.$.) $\cong \operatorname{hom}_{D}(M, D) \neq 0$. Let $Q \in D^{p \times m}$ be such that $\operatorname{ker}_{D}(R)=.Q D^{m}$. Since $R Q=0$, we get the following complex:

$$
D^{1 \times q} \stackrel{\cdot R}{\longrightarrow} D^{1 \times p} \stackrel{\cdot Q}{\longrightarrow} D^{1 \times m} .
$$

Lemma 3 ([11]). With the above notations, we have:

$$
\left\{\begin{array}{l}
t(M)=\operatorname{ker}_{D}(. Q) / \operatorname{im}_{D}(. R), \\
M / t(M) \cong D^{1 \times p} / \operatorname{ker}_{D}(. Q) .
\end{array}\right.
$$

In particular, $\pi(\lambda)$ is a torsion element of $M$ if and only if $\lambda Q=0$.

$\mathrm{RR} \mathrm{n}^{\circ} 8225$ 
Remark 1. Using Lemma $2, \varphi \in \operatorname{hom}_{D}(M, D)$ has the form $\varphi_{\mu}$ for a certain $\mu \in \operatorname{ker}_{D}(R$. $)=$ $\operatorname{im}_{D}\left(Q\right.$.), i.e., $\mu=Q \xi$ for some $\xi \in D^{m}$. Hence, if $\pi(\lambda) \in M \backslash t(M)$, i.e., $\lambda Q \neq 0$ by Lemma 3, there exists $\xi \in D^{m}$ such that $\lambda Q \xi \neq 0$. Thus, $\mu:=Q \xi \in \operatorname{ker}_{D}\left(R\right.$.) yields $\varphi_{\mu} \in \operatorname{hom}_{D}(M, D)$ such that $\varphi_{\mu}(\pi(\lambda)) \neq 0$.

The problem of finding a unimodular element $m^{\star}=\pi\left(\lambda^{\star}\right)$ of $M$ and $\mu^{\star}=Q \xi^{\star} \in \operatorname{ker}_{D}(R$. $)$ such that $\varphi_{\mu^{\star}} \in \operatorname{hom}_{D}(M, D)$ satisfies $\varphi_{\mu^{\star}}\left(m^{\star}\right)=1$ amounts to solving the following problem:

Problem 1. Find $\lambda^{\star} \in D^{1 \times p}$ and $\xi^{\star} \in D^{m}$ such that $\lambda^{\star} Q \xi^{\star}=1$.

Remark 2. We note that Problem 1 corresponds to solving a certain inhomogeneous quadratic equation in the $\lambda_{i}^{\star}$ 's and the $\xi_{j}^{\star}$ 's. As for the problem of checking whether or not $\pi\left(\lambda^{\star}\right)$ is a unimodular element of $M$, it is a linear problem: Check whether or not $\lambda^{\star} Q \in D^{1 \times m}$ admits a right inverse in $D$. For instance, this can be answered constructively for (not necessarily commutative) polynomial rings which admit Gröbner basis techniques (see, e.g., [11, 12]).

If one entry of $Q$ is invertible in $D$, then Problem 1 can be solved easily: if $Q_{i j} \in \mathrm{U}(D)$ and $\left\{f_{r}\right\}_{r=1, \ldots, p}$ (resp., $\left\{h_{s}\right\}_{s=1, \ldots, m}$ ) is the standard basis of $D^{1 \times p}$ (resp., $D^{m}$ ), then $\lambda^{\star}=f_{i}$ and $\xi^{\star}=Q_{i j}^{-1} h_{j}$ are such that $\lambda^{\star} Q \xi^{\star}=1$. Then, $m^{\star}=\pi\left(f_{i}\right)$ is a unimodular element of $M$, $\mu^{\star}=Q \xi^{\star} \in \operatorname{ker}_{D}(R$.$) , and \varphi_{\mu^{\star}}\left(m^{\star}\right)=1$.

More generally, if one row (resp., one column) of $Q$ admits a right inverse (resp., a left inverse) over $D$, then Problem 1 can be solved easily. For instance, if the $j^{\text {th }}$ column $Q \bullet j$ of $Q$ admits a left inverse $T \in D^{1 \times p}$, then considering $\lambda^{\star}=T$ and $\xi^{\star}=h_{j}$, where $\left\{h_{s}\right\}_{s=1, \ldots, m}$ is the standard basis of $D^{m}$, and $\mu^{\star}=Q \xi^{\star}$, we get $\lambda^{\star} \mu^{\star}=1$, which proves that $m^{\star}=\pi(T) \in \mathrm{U}(M)$ and $\varphi_{\mu^{\star}}\left(m^{\star}\right)=1$. Now, if the $i^{\text {th }}$ row $Q_{i}$ of $Q$ admits a right inverse $S \in D^{m}$, then considering $\lambda^{\star}=f_{i}$, where $\left\{f_{r}\right\}_{r=1, \ldots, p}$ is the standard basis of $D^{1 \times p}, \xi^{\star}=S$, and $\mu^{\star}=Q S \in \operatorname{ker}_{D}(R$.), we get $\lambda^{\star} \mu^{\star}=1$, which shows that $m^{\star}=\pi\left(\lambda^{\star}\right) \in \mathrm{U}(M)$ and $\varphi_{\mu^{\star}}\left(m^{\star}\right)=1$.

Let us illustrate these results with two explicit examples.

Example 2. Let us consider the Weyl algebra $D=A_{3}(\mathbb{Q})$ and the finitely presented left $D$-module $M=D^{1 \times 3} /\left(D^{1 \times 3} R\right)$, where the matrix $R$ is defined by:

$$
R=\left(\begin{array}{ccc}
\frac{1}{2} x_{2} \partial_{1} & x_{2} \partial_{2}+1 & x_{2} \partial_{3}+\frac{1}{2} \partial_{1} \\
-\frac{1}{2} x_{2} \partial_{2}-\frac{3}{2} & 0 & \frac{1}{2} \partial_{2} \\
-\partial_{1}-\frac{1}{2} x_{2} \partial_{3} & -\partial_{2} & -\frac{1}{2} \partial_{3}
\end{array}\right) \in D^{3 \times 3} .
$$

The corresponding system defines the infinitesimal transformations of the Lie pseudogroup formed by the contact transformations (see Example V.1.84 in [31]). We can check that $\operatorname{ker}_{D}(R)=.Q D$, where the matrix $Q \in D^{3}$ is defined by:

$\mathrm{RR} \mathrm{n}^{\circ} 8225$

$$
Q=\left(\begin{array}{c}
-\partial_{2} \\
\partial_{1}+x_{2} \partial_{3} \\
-x_{2} \partial_{2}-2
\end{array}\right)
$$


Moreover, $Q$ admits the left inverse $T=\frac{1}{2}\left(\begin{array}{lll}x_{2} & 0 & -1\end{array}\right)$. Thus, if $\lambda^{\star}=T$ and $\xi^{\star}=1$, i.e., $\mu^{\star}=Q$, then $\lambda^{\star} \mu^{\star}=1$, which shows that $m^{\star}=\pi\left(\lambda^{\star}\right)$ is a unimodular element of $M$ and $\varphi_{\mu^{\star}}\left(m^{\star}\right)=1$. Following [11], we can prove that $M$ is a torsion-free left $D$-module, i.e., $t(M)=0$. Then, using Lemma 3, we get $\operatorname{ker} \varphi_{\mu^{\star}}=\operatorname{ker}_{D}(. Q) / \operatorname{im}_{D}(. R)=t(M)=0$, i.e., $\varphi_{\mu^{\star}}$ is injective. Thus, $M=D m^{\star} \oplus \operatorname{ker} \varphi_{\mu^{\star}}=D m^{\star} \cong D$, which proves that $M$ is a free left $D$-module of rank one (compare with Example 1.5.1 of [33]).

Example 3. Let $D=A_{3}(\mathbb{Q})$ be the Weyl algebra and $M=D^{1 \times 3} /(D R)$ the left $D$-module finitely presented by $R=\left(\begin{array}{lll}\partial_{1}+x_{2} & \partial_{2} & \partial_{3}+x_{1}\end{array}\right)$. Using the OrEMoDULES package ([12]), we get $\operatorname{ker}_{D}(R)=.Q D^{4}$, where the matrix $Q \in D^{3 \times 4}$ is defined by:

$$
Q=\left(\begin{array}{cccc}
\partial_{2}+\partial_{3}+x_{1} & -\left(\partial_{3}+x_{1}\right)^{2} & -\left(\partial_{1}+x_{2}\right)\left(\partial_{3}+x_{1}\right)+2 & 0 \\
-\left(\partial_{1}+x_{2}\right) & 0 & 0 & \partial_{3}+x_{1} \\
-\left(\partial_{1}+x_{2}\right) & \left(\partial_{1}+x_{2}\right)\left(\partial_{3}+x_{1}\right)+1 & \left(\partial_{1}+x_{2}\right)^{2} & -\partial_{2}
\end{array}\right)
$$

We can check that the last row of $Q$ admits the right inverse $S=\left(\begin{array}{llll}\partial_{3}+x_{1} & 1 & 0 & 0\end{array}\right)^{T}$. Thus, if $\lambda^{\star}=\left(\begin{array}{lll}0 & 0 & 1\end{array}\right), \xi^{\star}=S$, and $\mu^{\star}=Q S=\left(\partial_{2}\left(\partial_{3}+x_{1}\right)-\left(\partial_{1}+x_{2}\right)\left(\partial_{3}+x_{1}\right) 1\right)^{T}$, then $\lambda^{\star} \mu^{\star}=1$ and $R \mu^{\star}=0$. Hence, $m^{\star}=\pi\left(\lambda^{\star}\right) \in \mathrm{U}(M)$ and $\varphi_{\mu^{\star}}\left(m^{\star}\right)=1$. Therefore, $M=D m^{\star} \oplus \operatorname{ker} \varphi_{\mu^{\star}}$ and $\operatorname{ker} \varphi_{\mu^{\star}}=\operatorname{ker}_{D}\left(. \mu^{\star}\right) / \operatorname{im}_{D}(. R)=\left(D^{1 \times 2} T\right) /(D R)$, where:

$$
T=\left(\begin{array}{ccc}
1 & 0 & -\partial_{2}\left(\partial_{3}+x_{1}\right) \\
0 & 1 & \left(\partial_{1}+x_{2}\right)\left(\partial_{3}+x_{1}\right)
\end{array}\right) .
$$

Since $\operatorname{ker}_{D}(. T)=0$ and $R=C T$, where $C=\left(\partial_{1}+x_{2} \quad \partial_{2}\right)$, Proposition 1 then shows that $\operatorname{ker} \varphi_{\mu^{\star}} \cong O:=D^{1 \times 2} /(D C)$, which proves that $M \cong D \oplus O$. Now, since $\operatorname{rank}_{D}(M)=2$ and $\left(\partial_{3}+x_{1} \quad 0 \quad-\left(\partial_{1}+x_{2}\right)\right)^{T}$ is a right inverse of $R, M=D^{1 \times 3} /(D R)$ is a stably free left $D$-module of rank two (see [36] for more details), and thus a free left $D$-module of rank two by Stafford's theorem (see 4 of Theorem 1 ). Therefore, we get $D \oplus O \cong D^{1 \times 2}$, which is consistent with the fact that $O$ is a stably free left $D$-module of rank one since $\left(\begin{array}{ll}-\partial_{2} & \partial_{1}+x_{2}\end{array}\right)^{T}$ is a right inverse of $C$. Finally, following [36], we can prove that $O$ is not a free left $D$-module of rank one, which shows that $D \oplus O \cong D^{1 \times 2}$ does not imply $O \cong D$.

In Section 3.3, we shall explain how unimodular elements of a finitely generated left $D$ module $M$ of rank at least 2 can be computed when $D$ is a very simple noetherian domain, a concept studied in the next section. In particular, in Section 3.2, it is shown that the Weyl algebras $A_{n}(k)$ and $B_{n}(k)$ satisfy this interesting property.

\subsection{Very simple domains}

Let us now introduce an important definition for what follows.

Definition 3 ([18]). A ring $D$ is called very simple if $D$ is prime, namely, $d_{1} D d_{2} \neq 0$ for all $d_{1}, d_{2} \in D \backslash\{0\}$, noetherian and satisfies the following property:

$$
\forall a, b, c \in D, \forall d \in D \backslash\{0\}, \exists u, v \in D: D a+D b+D c=D(a+d u c)+D(b+d v c) .
$$


If $D$ is a very simple ring, choosing $a=b=0, c=1$, and $d \in D \backslash\{0\}$, there exist $u, v \in D$ such that $D=D d u+D d v$, which implies that there exist $s, t \in D$ such that $1=s d u+t d v$, and thus $D d D=D$. Hence, every two-sided ideal of $D$ is trivial, i.e., $D$ is a simple ring (see, e.g., $[30,38])$.

Moreover, considering $d=1$ in (6), every left ideal of $D$ generated by three elements, and thus every finitely generated left ideal of $D$, can be generated by two elements.

Clearly, a domain is prime. A well-known result states that a left (resp., right) noetherian domain satisfies the left (resp., right) Ore condition (see, e.g., $[24,30]$ ). Hence, if $D$ is a very simple domain and $d_{1}, d_{2} \in D \backslash\{0\}$, there exist $e_{1}, e_{2} \in D \backslash\{0\}$ satisfying $d:=d_{1} e_{1}=d_{2} e_{2}$ (see Section 2). Thus, (6) yields:

$$
D a+D b+D c=D(a+d u c)+D(b+d v c)=D\left(a+d_{1}\left(e_{1} u\right) c\right)+D\left(b+d_{2}\left(e_{2} v\right) c\right) .
$$

Lemma 4 ([18]). If $D$ is a very simple domain, then we have:

1. Every finitely generated left ideal of $D$ can be generated by two elements.

2. The following property holds:

$\forall a, b, c \in D, \forall d_{1}, d_{2} \in D \backslash\{0\}, \exists u, v \in D: D a+D b+D c=D\left(a+d_{1} u c\right)+D\left(b+d_{2} v c\right)$.

Remark 3. If $D$ is a very simple domain admitting an involution $\theta$, namely, an antiautomorphism of order two, i.e., an additive map $\theta: D \longrightarrow D$ satisfying $\theta\left(d_{1} d_{2}\right)=\theta\left(d_{2}\right) \theta\left(d_{1}\right)$ for all $d_{1}, d_{2} \in D$, and $\theta^{2}=\mathrm{id}_{D}$, then a right analogue of (7) holds, namely:

$\forall a, b, c \in D, \forall d_{1}, d_{2} \in D \backslash\{0\}, \exists u, v \in D: a D+b D+c D=\left(a+c u d_{1}\right) D+\left(b+c v d_{2}\right) D$.

Indeed, applying (7) to $\theta(a), \theta(b), \theta(c) \in D$ and $\theta\left(d_{1}\right), \theta\left(d_{2}\right) \in D \backslash\{0\}$, there exist $u^{\prime}, v^{\prime} \in D$ such that $D \theta(a)+D \theta(b)+D \theta(c)=D\left(\theta(a)+\theta\left(d_{1}\right) u^{\prime} \theta(c)\right)+D\left(\theta(b)+\theta\left(d_{2}\right) v^{\prime} \theta(c)\right)$, i.e.,

$$
\left\{\begin{array}{l}
\theta(a)=\alpha_{1}\left(\theta(a)+\theta\left(d_{1}\right) u^{\prime} \theta(c)\right)+\alpha_{2}\left(\theta(b)+\theta\left(d_{2}\right) v^{\prime} \theta(c)\right), \\
\theta(b)=\beta_{1}\left(\theta(a)+\theta\left(d_{1}\right) u^{\prime} \theta(c)\right)+\beta_{2}\left(\theta(b)+\theta\left(d_{2}\right) v^{\prime} \theta(c)\right), \\
\theta(c)=\gamma_{1}\left(\theta(a)+\theta\left(d_{1}\right) u^{\prime} \theta(c)\right)+\gamma_{2}\left(\theta(b)+\theta\left(d_{2}\right) v^{\prime} \theta(c)\right),
\end{array}\right.
$$

for certain $\alpha_{1}, \alpha_{2}, \beta_{1}, \beta_{2}, \gamma_{1}, \gamma_{2} \in D$. Hence, if $u=\theta\left(u^{\prime}\right)$ and $v=\theta\left(v^{\prime}\right)$, then we obtain

$$
\left\{\begin{array}{l}
a=\left(a+c u d_{1}\right) \theta\left(\alpha_{1}\right)+\left(b+c v d_{2}\right) \theta\left(\alpha_{2}\right), \\
b=\left(a+c u d_{1}\right) \theta\left(\beta_{1}\right)+\left(b+c v d_{2}\right) \theta\left(\beta_{2}\right), \\
c=\left(a+c u d_{1}\right) \theta\left(\gamma_{1}\right)+\left(b+c v d_{2}\right) \theta\left(\gamma_{2}\right),
\end{array}\right.
$$

and proves (8). Finally, we note that the ring $D=A\left\langle\partial_{1}, \ldots, \partial_{n}\right\rangle$ of PD operators admits the standard involution $\theta$ defined by:

$$
\forall a \in A, \quad \theta(a)=a, \quad \theta\left(\partial_{i}\right)=-\partial_{i}, \quad i=1, \ldots, n .
$$

$\mathrm{RR} \mathrm{n}^{\circ} 8225$ 
Theorem 2 ([42]). The Weyl algebra $A_{n}(k)$ is a very simple domain.

The computation of elements $u$ and $v$ defined in (7) is implemented in the STAFFord package ([36]) based on algorithms developed in [21, 25] for the computation of two generators of left/right ideals generated by three elements.

Example 4. Let us consider $D=A_{2}(\mathbb{Q}), a=\partial_{1}, b=\partial_{2}, c=x_{1}, d_{1}=x_{2}$, and $d_{2}=x_{1}$. If $u=0$ and $v=1$, and $a_{2}=a+d_{1} u c=\partial_{1}$ and $b_{2}=b+d_{2} v c=\partial_{2}+x_{1}^{2}$, then (7) holds, i.e.,

$$
\left\{\begin{array}{l}
a=a_{2}, \\
b=\frac{1}{2} x_{1}\left(\partial_{2}+x_{1}^{2}\right) a_{2}+\frac{1}{2}\left(-x_{1} \partial_{1}+2\right) b_{2}, \\
c=-\frac{1}{2}\left(\partial_{2}+x_{1}^{2}\right) a_{2}+\frac{1}{2} \partial_{1} b_{2},
\end{array}\right.
$$

which shows that $D a+D b+D c=D a_{2}+D b_{2}$. Finally, using the involution $\theta$ of $D$ defined by (9), the above identities yield $\theta(a) D+\theta(b) D+\theta(c) D=\theta\left(a_{2}\right) D+\theta\left(b_{2}\right) D$, where $\theta(a)=\theta\left(a_{2}\right)=-\partial_{1}, \theta(b)=-\partial_{2}, \theta(c)=x_{1}$, and $\theta\left(b_{2}\right)=-\partial_{2}+x_{1}^{2}$.

Stafford's theorem, namely, Theorem 2, has recently been extended. Let us briefly review these extensions.

We denote by $\operatorname{Kdim}(D)$ the Krull dimension of a noetherian ring $D([30])$.

Theorem 3 (Proposition 1.3 of [18]). If $D$ is a simple noetherian ring of Krull dimension 1 , then $D$ is very simple.

A ring $D$ is artinian if every descending chain of ideals of $D$ is stationary (see, e.g., [38]).

Proposition 2 ((ii) of Corollary 6.6.7 of [30]). Let $A$ be a noetherian differential ring with a finite Krull dimension and $D=A\langle\partial\rangle$ the ring of $O D$ operators with coefficients in $A$. If $A$ is not artinian and $D$ is simple, then $\operatorname{Kdim}(D)=\operatorname{Kdim}(A)$.

Theorem 4 ([36]). The ring $A\langle\partial\rangle$ of $O D$ operators with coefficients in the differential ring $A=k \llbracket t \rrbracket$ (resp., $k\{t\}$, where $k \in\{\mathbb{R}, \mathbb{C}\}$ ) is a very simple domain.

Proof. Let $A$ be either $k \llbracket t \rrbracket$ or $k\{t\}$ with $k \in\{\mathbb{R}, \mathbb{C}\}$ in the latter case. The ring $A$ is not artinian because $(t) \supset\left(t^{2}\right) \supset\left(t^{3}\right) \supset \ldots$ is an infinite strictly descending chain of ideals, i.e., there exists no $I \in \mathbb{Z}_{\geq 0}$ such that $\left(t^{i}\right)=\left(t^{i+1}\right)$ for all $i \geq I$. It is also well-known that $A$ is a local ring, namely, $\mathfrak{m}:=(t)$ is the only maximal ideal of $A$ (namely, the only ideal $\mathfrak{m}$ of $A$ such that $A / \mathfrak{m}$ is a field), and $D=A\langle\partial\rangle$ is a simple noetherian domain (see, e.g., [6, 28]).

Now, if $A$ is a local ring, $\mathfrak{m}$ the maximal ideal of $A$, and $k:=A / \mathfrak{m}$ the residue field, then a standard result of commutative algebra asserts that $\operatorname{Kdim}(A) \leq \operatorname{dim}_{k}\left(\mathfrak{m} / \mathfrak{m}^{2}\right)$. See, e.g., [38], p. 487. Since $\operatorname{dim}_{k}\left((t) /\left(t^{2}\right)\right)=1$, we get $\operatorname{Kdim}(A) \leq 1$. Moreover, since an integral domain is a field if and only if its Krull dimension is 0 and the integral domain $A$ is not a field, $\operatorname{Kdim}(A)=1$, which yields $\operatorname{Kdim}(D)=1$ by Proposition 2 and finally proves the result by Theorem 3 .

$\mathrm{RR} \mathrm{n}^{\circ} 8225$ 
Theorem 5 ([9]). Let $A=k\left(\left(x_{1}, \ldots, x_{n}\right)\right.$ ) (resp., $k\left\{\left\{x_{1}, \ldots, x_{n}\right\}\right\}$ ) be the field of fractions of the ring of formal power series (resp., the ring of locally convergent power series) with coefficients in $k$ (resp., $k \in\{\mathbb{R}, \mathbb{C}\}$ ). Then, the ring $A\left\langle\partial_{1}, \ldots, \partial_{n}\right\rangle$ of PD operators with coefficients in $A$ is a very simple domain.

A consequence of $D$ being very simple is that the stable rank of $D$ is at most two, i.e., $\operatorname{sr}(D) \leq 2$. See, e.g., [18, 30, 36]. This implies the following theorem.

Theorem 6 ([18, 36, 30]). Let $D$ be a very simple domain and $M$ a finitely generated stably free left $D$-module. If $\operatorname{rank}_{D}(M) \geq 2$, then $M$ is free. If $\operatorname{rank}_{D}(M)=1$, then $M$ is isomorphic to a left ideal of $D$ which can be generated by two elements.

In [36], we explained how to compute bases of a finitely generated left/right projective $D$-module $M$ with $\operatorname{rank}_{D}(M) \geq \operatorname{sr}(D)$. The corresponding algorithm is implemented in the STAFFORD package $([36])$ for $D=A_{n}(\mathbb{Q})$ (see 4 of Theorem 1). Using Theorems 4 and 5 , the same algorithm is valid for $D=A\langle\partial\rangle$, where $A=k \llbracket t \rrbracket$ or $A=k\{t\}$ and $k \in\{\mathbb{R}, \mathbb{C}\}$, and for $D=A\left\langle\partial_{1}, \ldots, \partial_{n}\right\rangle$, where $A=k\left(\left(x_{1}, \ldots, x_{n}\right)\right)$ or $A=k\left\{\left\{x_{1}, \ldots, x_{n}\right\}\right\}$ with $k \in\{\mathbb{R}, \mathbb{C}\}$. For more details, see [37].

If $D$ is a very simple domain and if we consider the particular case $a=b=0, c=1$, and $d_{1}, d_{2} \in D \backslash\{0\}$ in (7), then there exist $u, v \in D$ such that $D d_{1} u+D d_{2} v=D$, i.e., such that $y d_{1} u+z d_{2} v=1$ holds for some $y, z \in D$. We obtain the following corollary.

Corollary 1 ([42]). Let $D$ be a very simple domain and $d_{1}, d_{2} \in D \backslash\{0\}$. Then, the following inhomogeneous quadratic equation

$$
y_{1} d_{1} z_{1}+y_{2} d_{2} z_{2}=1
$$

admits a solution $\left(y_{1} y_{2} z_{1} z_{2}\right)^{T} \in D^{4}$.

We emphasize that the algorithmization of Stafford's theorems stated in Section 1, which hold for a very simple domain $D$ as we shall see in the sequel, is based on the solvability of (10). Hence, Corollary 1 plays a key role in the constructive versions of Stafford's theorems for very simple domains.

Elements $y_{1}, y_{2}, z_{1}$, and $z_{2}$ as in Corollary 1 can be computed by the STAFFord package for $D=A_{n}(\mathbb{Q})$ ([36]). However, due to involved computations required in the corresponding algorithm, solutions of the quadratic equation (10) can in general only be computed in reasonable time for low order and low degree PD operators $d_{1}$ and $d_{2}$.

\subsection{Computation of unimodular elements}

The next theorem, called Serre's splitting-off theorem due to its reminiscence of [39], states the existence of a unimodular element in a finitely generated module of sufficiently big rank.

$\mathrm{RR} \mathrm{n}^{\circ} 8225$ 
Theorem 7 ([42]). Let $D$ be a very simple domain and $M$ a finitely generated left $D$-module. Then there exists $r \in \mathbb{Z}_{\geq 0}$ and a finitely generated left $D$-module $M^{\prime}$ with $\operatorname{rank}_{D}\left(M^{\prime}\right) \leq 1$ such that:

$$
M \cong D^{1 \times r} \oplus M^{\prime}
$$

If $M$ is torsion-free, then $M^{\prime}$ can be chosen as a finitely generated left ideal of $D$ (which can be generated by two elements).

Proof. Since $M$ is a finitely generated left $D$-module over a left noetherian domain $D, M$ is finitely presented, i.e., there exists $R \in D^{q \times p}$ such that $M=D^{1 \times p} /\left(D^{1 \times q} R\right)$. For more details, see, e.g., [38]. Let $\pi: D^{1 \times p} \longrightarrow M$ be the canonical projection onto $M$.

If $\operatorname{rank}_{D}(M) \leq 1$, then the result trivially holds with $r=0$ and $M^{\prime}=M$.

Let us now suppose that $\operatorname{rank}_{D}(M) \geq 2$. Then, by $1 \Rightarrow 3$ of Lemma $1, M$ is not a torsion left $D$-module, and thus there exists $m_{1} \notin t(M)$. Moreover, since $M$ is not a torsion left $D$-module, $\operatorname{ker}_{D}(R$. $) \cong \operatorname{hom}_{D}(M, D) \neq 0$ by $2 \Rightarrow 1$ of Lemma 1 . Let $Q \in D^{p \times m}$ be such that $\operatorname{ker}_{D}(R)=.Q D^{m}$. According to Lemma $3, m_{1}=\pi\left(\lambda_{1}\right)$ has to be chosen so that $\lambda_{1} Q \neq 0$.

Using Remark 1, there exists $\mu_{1} \in \operatorname{ker}_{D}(R)=.Q D^{m}$ such that $\varphi_{1}:=\varphi_{\mu_{1}}$ satisfies $\varphi_{1}\left(m_{1}\right) \neq 0$. In particular, $\mu_{1}=Q \xi_{1}$ has to be chosen so that $\left(\lambda_{1} Q\right) \xi_{1} \neq 0$. Then, the following diagram is commutative with exact rows:

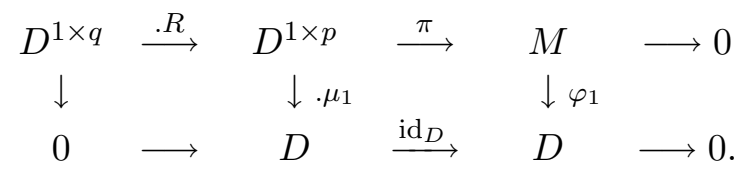

We can easily check that $\operatorname{ker} \varphi_{1}=\operatorname{ker}_{D}\left(. \mu_{1}\right) /\left(D^{1 \times q} R\right)$. For more details, see [13]. Since $0 \neq D \varphi_{1}\left(m_{1}\right) \subseteq \varphi_{1}(M)=D^{1 \times p} \mu_{1} \subseteq D$ and $D$ is a noetherian domain, $\operatorname{rank}_{D}\left(\varphi_{1}(M)\right)=1$. Then, the short exact sequence $0 \longrightarrow \operatorname{ker} \varphi_{1} \longrightarrow M \longrightarrow \operatorname{im} \varphi_{1} \longrightarrow 0$ yields:

$$
\operatorname{rank}_{D}\left(\operatorname{ker} \varphi_{1}\right)=\operatorname{rank}_{D}(M)-\operatorname{rank}_{D}\left(\operatorname{im} \varphi_{1}\right) \geq 1 .
$$

For more details, see $[30,38]$. Thus, by $1 \Rightarrow 3$ of Lemma 1 , $\operatorname{ker} \varphi_{1}$ is not a torsion left $D$-module, and there exists $m_{2} \in \operatorname{ker} \varphi_{1}$ such that $m_{2} \notin t(M)$. We choose $m_{2}$ as follows. Let $S \in D^{r \times p}$ be such that $\operatorname{ker}_{D}\left(. \mu_{1}\right)=D^{1 \times r} S$. If $\nu \in D^{1 \times r}$ satisfies $\nu(S Q) \neq 0$ and $\lambda_{2}=\nu S$, then Lemma 3 shows that we can take $m_{2}=\pi\left(\lambda_{2}\right)$. Let $\xi_{2} \in D^{m}$ be such that $\left(\lambda_{2} Q\right) \xi_{2} \neq 0$ and define $\mu_{2}=Q \xi_{2}$. Then $\varphi_{2}:=\varphi_{\mu_{2}} \in \operatorname{hom}_{D}(M, D)$ satisfies $\varphi_{2}\left(m_{2}\right) \neq 0$. Since $m_{2} \in \operatorname{ker} \varphi_{1}$, we have $\varphi_{1}\left(m_{2}\right)=0$.

If $\varphi_{2}\left(m_{1}\right) \neq 0$, then by the right Ore property of $D, r_{1}, r_{2} \in D \backslash\{0\}$ exist such that:

$$
\varphi_{1}\left(m_{1}\right) r_{1}+\varphi_{2}\left(m_{1}\right) r_{2}=0 \text {. }
$$

$\mathrm{RR} \mathrm{n}^{\circ} 8225$ 
Recall that $\operatorname{hom}_{D}(M, D)$ has a right $D$-module structure defined by $(\varphi r)(m)=\varphi(m) r$ for all $r \in D$ and for all $m \in M$ (see, e.g., [38]). Then $\varphi_{2}^{\prime}=\varphi_{1} r_{1}+\varphi_{2} r_{2} \in \operatorname{hom}_{D}(M, D)$ satisfies:

$$
\left\{\begin{array}{l}
\varphi_{2}^{\prime}\left(m_{1}\right)=\varphi_{1}\left(m_{1}\right) r_{1}+\varphi_{2}\left(m_{1}\right) r_{2}=0, \\
\varphi_{2}^{\prime}\left(m_{2}\right)=\varphi_{1}\left(m_{2}\right) r_{1}+\varphi_{2}\left(m_{2}\right) r_{2}=\varphi_{2}\left(m_{2}\right) r_{2} \neq 0 .
\end{array}\right.
$$

Therefore, without loss of generality, we may assume that $\varphi_{2}\left(m_{1}\right)=0$. Then, we have:

$$
\varphi_{1}\left(m_{1}\right) \neq 0, \quad \varphi_{1}\left(m_{2}\right)=0, \quad \varphi_{2}\left(m_{1}\right)=0, \quad \varphi_{2}\left(m_{2}\right) \neq 0 .
$$

Applying Corollary 1 to $d_{1}:=\varphi_{1}\left(m_{1}\right) \neq 0$ and $d_{2}:=\varphi_{2}\left(m_{2}\right) \neq 0$, there exist $y_{1}, y_{2}, z_{1}, z_{2} \in D$ such that (10) holds, i.e., $y_{1} \varphi_{1}\left(m_{1}\right) z_{1}+y_{2} \varphi_{2}\left(m_{2}\right) z_{2}=1$, or equivalently:

$$
y_{1}\left(\lambda_{1} \mu_{1}\right) z_{1}+y_{2}\left(\lambda_{2} \mu_{2}\right) z_{2}=1 \text {. }
$$

Let $\lambda^{\star}=y_{1} \lambda_{1}+y_{2} \lambda_{2} \in D^{1 \times p}, m^{\star}=\pi\left(\lambda^{\star}\right)=y_{1} m_{1}+y_{2} m_{2} \in M, \mu^{\star}=\mu_{1} z_{1}+\mu_{2} z_{2} \in$ $\operatorname{ker}_{D}(R$.$) , and \varphi:=\varphi_{1} z_{1}+\varphi_{2} z_{2}=\varphi_{\mu^{\star}} \in \operatorname{hom}_{D}(M, D)$. Then,

$$
\begin{aligned}
\varphi\left(m^{\star}\right) & =\left(\varphi_{1} z_{1}+\varphi_{2} z_{2}\right)\left(m^{\star}\right)=\varphi_{1}\left(m^{\star}\right) z_{1}+\varphi_{2}\left(m^{\star}\right) z_{2} \\
& =\left(y_{1} \varphi_{1}\left(m_{1}\right)+y_{2} \varphi_{1}\left(m_{2}\right)\right) z_{1}+\left(y_{1} \varphi_{2}\left(m_{1}\right)+y_{2} \varphi_{2}\left(m_{2}\right)\right) z_{2} \\
& =y_{1} \varphi_{1}\left(m_{1}\right) z_{1}+y_{2} \varphi_{2}\left(m_{2}\right) z_{2}=1,
\end{aligned}
$$

which shows that $m^{\star} \in \mathrm{U}(M)$. As explained at the beginning of Section 3.1, we then get $M=D m^{\star} \oplus \operatorname{ker} \varphi \cong D \oplus \operatorname{ker} \varphi$, where $\operatorname{ker} \varphi=\operatorname{ker}_{D}\left(. \mu^{\star}\right) /\left(D^{1 \times q} R\right)$ is a left $D$-submodule of $M$ with $\operatorname{rank}_{D}(\operatorname{ker} \varphi)=\operatorname{rank}_{D}(M)-1$.

Repeating the same arguments, we obtain $M \cong D^{1 \times r} \oplus M^{\prime}$, where $\operatorname{rank}_{D}\left(M^{\prime}\right) \leq 1$.

Finally, assuming $t(M)=0$, we have $t\left(M^{\prime}\right)=0$, and thus $M^{\prime}$ is a torsion-free left $D$ module of rank at most one. Using the concept of minimal parametrization ([11]), $M^{\prime}$ is then isomorphic to a finitely generated left ideal of $D$. Indeed, if $M^{\prime}=D^{1 \times l} /\left(D^{1 \times m} U\right)$ and $V \in D^{l}$ is such that $V \in \operatorname{ker}_{D}\left(U\right.$.), then Theorem 8 of [11] shows that $M^{\prime}$ is isomorphic to the left ideal of $D$ generated by the entries of $V$. Since $D$ is a very simple domain, $M^{\prime}$ can be generated by two elements by 1 of Lemma 4 .

We summarize the procedure indicated in the above proof in the following algorithm.

Algorithm 1 (UnimodularElement).

- Input: A very simple domain $D$ and $R \in D^{q \times p}$ such that the finitely presented left $D$-module $M=D^{1 \times p} /\left(D^{1 \times q} R\right)$ has rank at least two, i.e., $\operatorname{rank}_{D}(M) \geq 2$.

- Output: $\lambda^{\star} \in D^{1 \times p}$ and $\mu^{\star} \in \operatorname{ker}_{D}(R$. $)$ such that we have $m^{\star}=\pi\left(\lambda^{\star}\right) \in \mathrm{U}(M)$ and:

$$
\varphi_{\mu^{\star}}\left(m^{\star}\right)=1
$$

$\operatorname{RR} n^{\circ} 8225$ 
1. Compute $Q \in D^{p \times m}$ such that $\operatorname{ker}_{D}(R)=.Q D^{m}$.

2. Pick $\lambda_{1} \in D^{1 \times p}$ such that $\lambda_{1} Q \neq 0$.

3. Find $\xi_{1} \in D^{m}$ such that $\left(\lambda_{1} Q\right) \xi_{1} \neq 0$ and define $\mu_{1}=Q \xi_{1} \in \operatorname{ker}_{D}(R$.$) .$

4. Compute $S \in D^{r \times p}$ such that $\operatorname{ker}_{D}\left(. \mu_{1}\right)=D^{1 \times r} S$.

5. Pick $\nu \in D^{1 \times r}$ such that $\nu(S Q) \neq 0$ and define $\lambda_{2}=\nu S$.

6. Find $\xi_{2} \in D^{m}$ such that $\left(\lambda_{2} Q\right) \xi_{2} \neq 0$ and define $\mu_{2}=Q \xi_{2} \in \operatorname{ker}_{D}(R$.$) .$

7. If $\lambda_{1} \mu_{2} \neq 0$, then compute $r_{1}, r_{2} \in D \backslash\{0\}$ such that $\left(\lambda_{1} \mu_{1}\right) r_{1}+\left(\lambda_{1} \mu_{2}\right) r_{2}=0$ and replace $\mu_{2}$ by $\mu_{1} r_{1}+\mu_{2} r_{2}$.

8. Compute $y_{1}, y_{2}, z_{1}, z_{2} \in D$ such that $y_{1}\left(\lambda_{1} \mu_{1}\right) z_{1}+y_{2}\left(\lambda_{2} \mu_{2}\right) z_{2}=1$.

9. Return $\lambda^{\star}=y_{1} \lambda_{1}+y_{2} \lambda_{2} \in D^{1 \times p}$ and $\mu^{\star}=\mu_{1} z_{1}+\mu_{2} z_{2} \in \operatorname{ker}_{D}(R$.).

In the context of Theorem 7 , let us compute a finite presentation $R^{\prime}$ of the kernel of $\varphi=\varphi_{\mu_{1}} z_{1}+\varphi_{\mu_{2}} z_{2}$ and an isomorphism between $M$ and $D \oplus L$, where $L$ is the cokernel of the presentation matrix $R^{\prime}$. This isomorphism is used in order to iterate Algorithm 1 and to explicitly describe the left $D$-isomorphism $M \cong D^{1 \times r} \oplus M^{\prime}$.

Let $T \in D^{s \times p}$ be such that $\operatorname{ker}_{D}\left(. \mu^{\star}\right)=D^{1 \times s} T$. Thus, $\operatorname{ker} \varphi=\left(D^{1 \times s} T\right) /\left(D^{1 \times q} R\right)$. Moreover, let $T_{2} \in D^{t \times s}$ be such that $\operatorname{ker}_{D}(. T)=D^{1 \times t} T_{2}, C \in D^{q \times s}$ such that $R=C T$ and $R^{\prime}:=\left(\begin{array}{ll}C^{T} & T_{2}^{T}\end{array}\right)^{T}$. We define $O:=D^{1 \times s} /\left(D^{1 \times(q+t)} R^{\prime}\right)$ and the canonical projection $\kappa: D^{1 \times s} \longrightarrow O$ onto $O$. Then, Proposition 1 yields the following left $D$-isomorphism:

$$
\begin{aligned}
\phi: \operatorname{ker} \varphi & \longrightarrow O \\
\pi(\gamma T) & \longmapsto \kappa(\gamma) .
\end{aligned}
$$

If $Z:=\left(\begin{array}{ll}I_{q} & 0\end{array}\right)^{T} \in D^{(q+t) \times q}$, then we have

$$
R^{\prime} T=Z R,
$$

which yields the following commutative diagram with exact rows and columns, where $\iota$ : $\operatorname{ker} \varphi \longrightarrow M$ is the canonical injection:

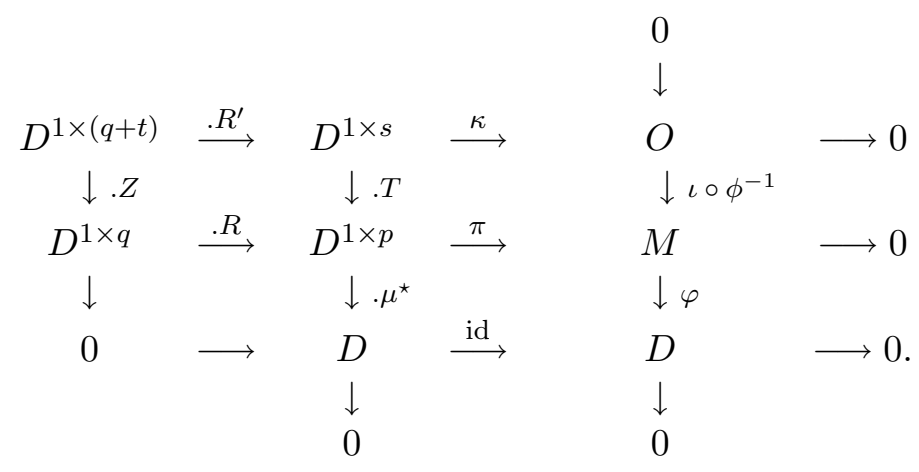

$\mathrm{RR} \mathrm{n}^{\circ} 8225$ 
Remark 4. We note that the short exact sequence $0 \longrightarrow \operatorname{ker}_{D}\left(. \mu^{\star}\right) \longrightarrow D^{1 \times p} \stackrel{. \mu^{\star}}{\longrightarrow} D \longrightarrow 0$ splits (see the paragraph after (3)), i.e., $D^{1 \times p} \cong D \oplus \operatorname{ker}_{D}\left(. \mu^{\star}\right)$, which shows that $\operatorname{ker}_{D}\left(. \mu^{\star}\right)$ is a stably free left $D$-module of rank $p-1$ (see Definition 1 ). If $\operatorname{ker}_{D}\left(. \mu^{\star}\right)$ is a free left $D$-module (e.g., if $D=A_{n}(k)$ and $p \geq 3$ by 4 of Theorem 1 ), then there exists a full row rank matrix $T \in D^{(p-1) \times p}$ (i.e., $\operatorname{ker}_{D}(. T)=0$ ) such that $\operatorname{ker}_{D}\left(. \mu^{\star}\right)=D^{1 \times(p-1)} T$, which yields $s=p-1$, $t=0$, and $R^{\prime}=C$ in the above diagram.

Now, using the split short exact sequence (3) and the decomposition (4), we get the following left $D$-isomorphism:

$$
\begin{aligned}
\iota_{1}: M & \longrightarrow D \oplus \operatorname{ker} \varphi \\
m=\pi(\lambda) & \longmapsto\left(\varphi(m), m-\varphi(m) m^{\star}\right)=\left(\lambda \mu^{\star}, \pi\left(\lambda\left(I_{p}-\mu^{\star} \lambda^{\star}\right)\right)\right) .
\end{aligned}
$$

Since we have $\varphi\left(m-\varphi(m) m^{\star}\right)=\lambda\left(I_{p}-\mu^{\star} \lambda^{\star}\right) \mu^{\star}=0$ for all $\lambda \in D^{1 \times p}, m=\pi(\lambda)$, and $\operatorname{ker}_{D}\left(. \mu^{\star}\right)=D^{1 \times s} T$, there exists $G \in D^{p \times s}$ such that:

$$
I_{p}-\mu^{\star} \lambda^{\star}=G T
$$

In other words, we have the following split exact sequence:

$$
D^{1 \times s} \underset{. G}{\stackrel{. T}{\rightleftarrows}} D^{1 \times p} \underset{. \lambda^{\star}}{\stackrel{. \mu^{\star}}{\rightleftarrows}} D \longrightarrow 0 \text {. }
$$

Now, using (11), we obtain the following left $D$-isomorphism

$$
\begin{aligned}
\iota_{2}: D \oplus \operatorname{ker} \varphi & \longrightarrow D \oplus O \\
(d, \pi(\gamma T)) & \longmapsto(d, \kappa(\gamma)),
\end{aligned}
$$

which combined with (13) (using (14)) yields the following left $D$-isomorphism:

$$
\begin{aligned}
\iota:=\iota_{2} \circ \iota_{1}: M & \longrightarrow D \oplus O \\
m=\pi(\lambda) & \longmapsto\left(\lambda \mu^{\star}, \kappa(\lambda G)\right) .
\end{aligned}
$$

To characterize $\iota^{-1}$, we first need to derive a few identities.

Multiplying $T$ (resp., $\lambda^{\star}$ ) from the left to (14) and using $T \mu^{\star}=0$ and $\lambda^{\star} \mu^{\star}=1$, we get:

$$
\begin{aligned}
T G T & =T-T \mu^{\star} \lambda^{\star}=T, \\
\lambda^{\star} G T & =\lambda^{\star}-\left(\lambda^{\star} \mu^{\star}\right) \lambda^{\star}=0 .
\end{aligned}
$$

(15) yields $\left(I_{s}-T G\right) T=0$ and using $\operatorname{ker}_{D}(. T)=D^{1 \times t} T_{2}$, there exists $E \in D^{s \times t}$ such that:

$$
I_{s}-T G=E T_{2} .
$$


Combining (16) and $\operatorname{ker}_{D}(. T)=D^{1 \times t} T_{2}$, there exists $F \in D^{1 \times t}$ such that:

$$
\lambda^{\star} G=F T_{2} .
$$

Now, multiplying $R$ from the left to (14), we obtain $R=R G T$ because $\mu^{\star} \in \operatorname{ker}_{D}(R$.). Using $R=C T$, we get $C T=R G T$, i.e., $(C-R G) T=0$ and using $\operatorname{ker}_{D}(. T)=D^{1 \times t} T_{2}$, there exists $H \in D^{q \times t}$ such that:

$$
C-R G=H T_{2} .
$$

Let us now introduce the following map:

$$
\begin{aligned}
\varpi: D \oplus O & \longrightarrow M \\
(d, \kappa(\gamma)) & \longmapsto \pi\left(d \lambda^{\star}+\gamma T\right) .
\end{aligned}
$$

We can easily check that $\varpi$ is a well-defined left $D$-homomorphism since, using (12), we have:

$$
\begin{aligned}
\forall \theta \in D^{1 \times(q+t)}, \quad \varpi\left(\left(d, \kappa\left(\gamma+\theta R^{\prime}\right)\right)\right) & =\pi\left(d \lambda^{\star}+\left(\gamma+\theta R^{\prime}\right) T\right)=\pi\left(d \lambda^{\star}+\gamma T+(\theta Z) R\right) \\
& =\pi\left(d \lambda^{\star}+\gamma T\right) .
\end{aligned}
$$

Then, using (14), $\lambda^{\star} \mu^{\star}=1, T \mu^{\star}=0,(17)$, and (18), we then have

$$
\begin{aligned}
(\varpi \circ \iota)(\pi(\lambda)) & =\varpi\left(\left(\lambda \mu^{\star}, \kappa(\lambda G)\right)=\pi\left(\lambda\left(\mu^{\star} \lambda^{\star}+G T\right)\right)=\pi(\lambda),\right. \\
(\iota \circ \varpi)((d, \kappa(\gamma))) & =\iota\left(\pi\left(d \lambda^{\star}+\gamma T\right)\right)=\left(\left(d \lambda^{\star}+\gamma T\right) \mu^{\star}, \kappa\left(\left(d \lambda^{\star}+\gamma T\right) G\right)\right) \\
& =\left(d, \kappa\left(d F T_{2}+\gamma\left(I_{s}-E T_{2}\right)\right)\right)=\left(d, \kappa\left(\gamma+(d F-\gamma E) T_{2}\right)\right)=(d, \kappa(\gamma)),
\end{aligned}
$$

which finally shows that $\iota^{-1}=\varpi$.

Let $\bar{R}=\left(\begin{array}{ll}0 & R^{\prime}\end{array}\right) \in D^{(q+t) \times(1+s)}, \bar{M}=D^{1 \times(1+s)} /\left(D^{1 \times(q+t)} \bar{R}\right)$ be the left $D$-module finitely presented by $\bar{R}$, and $\bar{\pi}=\mathrm{id}_{D} \oplus \kappa: D^{1 \times(1+s)} \longrightarrow \bar{M}$ the canonical projection onto $\bar{M}$. We get $\bar{M} \cong D \oplus O \cong M$. If $P_{1}:=\left(\begin{array}{ll}\mu^{\star} & G\end{array}\right) \in D^{p \times(1+s)}$ and $P_{2}=\left(\begin{array}{ll}I_{q} & -H\end{array}\right) \in D^{q \times(q+t)}$, then using (19), we get $R P_{1}=P_{2} \bar{R}$, which yields the following commutative diagram with exact rows:

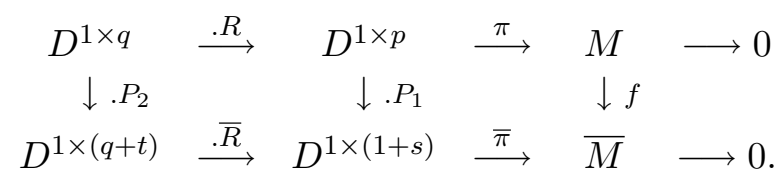

Hence, the left $D$-isomorphism $f$ is defined by:

$$
\begin{aligned}
f: M & \longrightarrow \bar{M} \\
\pi(\lambda) & \longmapsto \bar{\pi}\left(\lambda P_{1}\right)=\left(\lambda \mu^{\star}, \kappa(\lambda G)\right) .
\end{aligned}
$$

To finish, let us compute $f^{-1}$. Using the identity $R=C T$, we have:

$$
\bar{R}\left(\begin{array}{c}
\lambda^{\star} \\
T
\end{array}\right)=\left(\begin{array}{cc}
0 & C \\
0 & T_{2}
\end{array}\right)\left(\begin{array}{c}
\lambda^{\star} \\
T
\end{array}\right)=\left(\begin{array}{c}
C T \\
0
\end{array}\right)=\left(\begin{array}{c}
R \\
0
\end{array}\right)=\left(\begin{array}{c}
I_{q} \\
0
\end{array}\right) R=Z R .
$$

$\mathrm{RR} \mathrm{n}^{\circ} 8225$ 
Hence, if $P_{1}^{\prime}:=\left(\lambda^{\star T} \quad T^{T}\right)^{T} \in D^{(1+s) \times p}$ and $P_{2}^{\prime}=Z \in D^{(q+t) \times q}$, then we have $\bar{R} P_{1}^{\prime}=P_{2}^{\prime} R$, which yields the following commutative diagram with exact rows

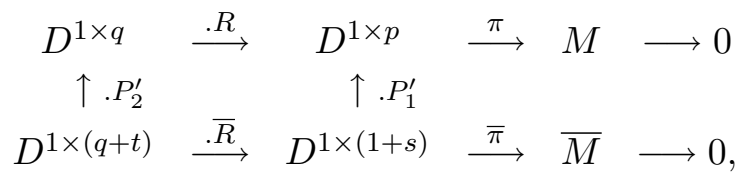

and the following left $D$-homomorphism:

$$
\begin{aligned}
g: \bar{M} & \longrightarrow M \\
\bar{\pi}\left(\left(\begin{array}{ll}
\theta_{1} & \left.\left.\theta_{2}\right)\right)
\end{array}\right.\right. & \longmapsto \pi\left(\left(\theta_{1} \quad \theta_{2}\right) P_{1}^{\prime}\right)=\pi\left(\theta_{1} \lambda^{\star}+\theta_{2} T\right) .
\end{aligned}
$$

Using (14), $\lambda^{\star} \mu^{\star}=1,(18), T \mu^{\star}=0,(17)$, and (19), we can easily check that

$$
\left\{\begin{aligned}
P_{1} P_{1}^{\prime} & =\mu^{\star} \lambda^{\star}+G T=I_{p}, \\
P_{1}^{\prime} P_{1} & =\left(\begin{array}{cc}
\lambda^{\star} \mu^{\star} & \lambda^{\star} G \\
T \mu^{\star} & T G
\end{array}\right)=\left(\begin{array}{cc}
1 & F T_{2} \\
0 & I_{s}-E T_{2}
\end{array}\right)=I_{s+1}+\left(\begin{array}{cc}
0 & F \\
0 & -E
\end{array}\right) \bar{R},
\end{aligned}\right.
$$

which yields $g \circ f=\mathrm{id}_{M}$ and $f \circ g=\mathrm{id}_{\bar{M}}$, and finally shows that $g=f^{-1}$.

Example 5. Let $D=A_{3}(\mathbb{Q})$ and $M=D^{1 \times 3} /(D R)$ be the left $D$-module finitely presented by the divergence operator in $\mathbb{R}^{3}$, namely, $R=\left(\begin{array}{lll}\partial_{1} & \partial_{2} & \partial_{3}\end{array}\right)$. Then, $\operatorname{rank}_{D}(M)=2$, which shows that there exists a non-trivial decomposition of $M$ as in Theorem 7 . We can check that $\operatorname{ker}_{D}(R)=.Q D^{3}$, where $Q$ is the matrix of PD operators defining the curl operator, namely:

$$
Q=\left(\begin{array}{ccc}
0 & -\partial_{3} & \partial_{2} \\
\partial_{3} & 0 & -\partial_{1} \\
-\partial_{2} & \partial_{1} & 0
\end{array}\right)
$$

Since the entries of $Q$ are not units of $D$ and none of the rows (resp., columns) of $Q$ admits a right inverse (resp., left inverse) over $D$, there is no easy way to detect unimodular elements of $M$. Then, let us apply Algorithm 1 to $M$. If we consider $\lambda_{1}=\left(\begin{array}{lll}0 & -1 & 0\end{array}\right)$, then $\lambda_{1} Q=\left(\begin{array}{lll}-\partial_{3} & 0 & \partial_{1}\end{array}\right) \neq 0$. Now, taking $\xi_{1}=\left(\begin{array}{lll}0 & 0 & 1\end{array}\right)^{T}$, then $\mu_{1}=Q \xi_{1}=\left(\begin{array}{lll}\partial_{2} & -\partial_{1} & 0\end{array}\right)^{T}$ and $d_{1}:=\lambda_{1} \mu_{1}=\partial_{1}$. Now, $\operatorname{ker}_{D}\left(. \mu_{1}\right)=D^{1 \times 2} S$, where $S$ and thus $S Q$ are defined by:

$$
S=\left(\begin{array}{ccc}
\partial_{1} & \partial_{2} & 0 \\
0 & 0 & 1
\end{array}\right), \quad S Q=\left(\begin{array}{ccc}
\partial_{2} \partial_{3} & -\partial_{1} \partial_{3} & 0 \\
-\partial_{2} & \partial_{1} & 0
\end{array}\right)
$$

Then, considering $\nu=\left(\begin{array}{ll}0 & 1\end{array}\right)$, we get $\nu(S Q) \neq 0$ and $\lambda_{2}=\nu S=\left(\begin{array}{lll}0 & 0 & 1\end{array}\right)$. The choice $\xi_{2}=\left(\begin{array}{lll}0 & 1 & 0\end{array}\right)^{T}$ yields $\mu_{2}=Q \xi_{2}=\left(\begin{array}{lll}-\partial_{3} & 0 & \partial_{1}\end{array}\right)^{T}$ and $d_{2}:=\lambda_{2} \mu_{2}=\partial_{1}$. Moreover, $\lambda_{1} \mu_{2}=0$. Computing a solution $\left(y_{1} y_{2} z_{1} z_{2}\right)^{T} \in D^{4}$ of $y_{1} d_{1} z_{1}+y_{2} d_{2} z_{2}=1$, we get:

$$
y_{1}=-x_{1}-1, \quad y_{2}=1, \quad z_{1}=1, \quad z_{2}=x_{1}+1 .
$$

$\mathrm{RR} \mathrm{n}^{\circ} 8225$ 
Thus, $\lambda^{\star}=y_{1} \lambda_{1}+y_{2} \lambda_{2}=\left(\begin{array}{lll}0 & x_{1}+1 & 1\end{array}\right)$ defines the unimodular element $m^{\star}=\pi\left(\lambda^{\star}\right)$ of $M$.

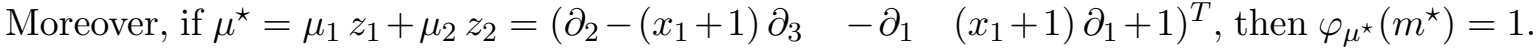
Hence, $M=D m^{\star} \oplus \operatorname{ker} \varphi_{\mu^{\star}}$, and $\operatorname{ker} \varphi_{\mu^{\star}}=\operatorname{ker}_{D}\left(. \mu^{\star}\right) /(D R)$. Remark 4 shows that $\operatorname{ker}_{D}\left(. \mu^{\star}\right)$ is a free left $D$-module of rank 2 and, computing a basis, we get $\operatorname{ker}_{D}\left(. \mu^{\star}\right)=D^{1 \times 2} T$, where:

$$
T=\left(\begin{array}{ccc}
1 & -\left(x_{1}+1\right)\left(\partial_{2}-\left(x_{1}+1\right) \partial_{3}\right) & \left(x_{1}+1\right) \partial_{3}-\partial_{2} \\
0 & \left(x_{1}+1\right) \partial_{1}+2 & \partial_{1}
\end{array}\right) .
$$

The matrix $T$ has full row rank, i.e., $\operatorname{ker}_{D}(. T)=0$. Let $C$ be such that $R=C T$, namely:

$$
C=\left(\partial_{1} \quad \partial_{2}-\left(x_{1}+1\right) \partial_{3}\right) .
$$

Then, $\operatorname{ker} \varphi_{\mu^{\star}} \cong O:=D^{1 \times 2} /(D C)$, which shows that $M \cong D \oplus O$. Let us give an explicit isomorphism. If $\bar{R}=\left(\begin{array}{ll}0 & C\end{array}\right) \in D^{1 \times 3}, \bar{M}=D^{1 \times 3} /(D \bar{R})$, and $\bar{\pi}: D^{1 \times 3} \longrightarrow \bar{M}$ the canonical projection onto $\bar{M}, P_{1}^{\prime}=\left(\lambda^{\star T} \quad T^{T}\right)^{T} \in D^{3 \times 3}$, then $\bar{R} P_{1}^{\prime}=C T=R$, which shows that the following diagram is commutative with exact rows

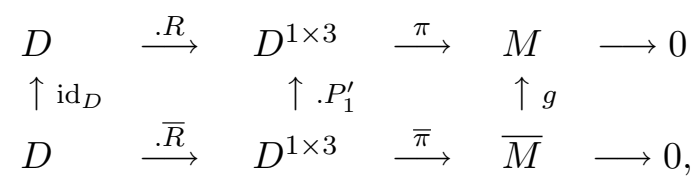

where $g(\bar{\pi}(\theta))=\pi\left(\theta P_{1}^{\prime}\right)$ for all $\theta \in D^{1 \times 3}$. Now, since $P_{1}^{\prime} \in \mathrm{GL}_{3}(D), g$ is a $D$-isomorphism and $f=g^{-1}$ is defined by $f(\pi(\lambda))=\bar{\pi}\left(\lambda P_{1}\right)$ for all $\lambda \in D^{1 \times 3}$, where:

$$
P_{1}:=P_{1}^{\prime-1}=\left(\begin{array}{ccc}
\partial_{2}-\left(x_{1}+1\right) \partial_{3} & 1 & 0 \\
-\partial_{1} & 0 & 1 \\
\left(x_{1}+1\right) \partial_{1}+1 & 0 & -\left(x_{1}+1\right)
\end{array}\right) .
$$

In other words, $\partial_{1} u_{1}+\partial_{2} u_{2}+\partial_{3} u_{3}=0$ is equivalent to $\partial_{1} w_{1}+\left(\partial_{2}-\left(x_{1}+1\right) \partial_{3}\right) w_{2}=0$ (and no condition on $v$ ) under the following invertible transformations:

$$
\begin{aligned}
& \left\{\begin{array}{l}
v=\left(x_{1}+1\right) u_{2}+u_{3}, \\
w_{1}=u_{1}-\left(x_{1}+1\right)\left(\partial_{2}-\left(x_{1}+1\right) \partial_{3}\right) u_{2}+\left(\left(x_{1}+1\right) \partial_{3}-\partial_{2}\right) u_{3}, \\
w_{2}=\left(\left(x_{1}+1\right) \partial_{1}+2\right) u_{2}+\partial_{1} u_{3},
\end{array}\right. \\
& \Leftrightarrow\left\{\begin{array}{l}
u_{1}=\left(\partial_{2}-\left(x_{1}+1\right) \partial_{3}\right) v+w_{1}, \\
u_{2}=-\partial_{1} v+w_{2} \\
u_{3}=\left(\left(x_{1}+1\right) \partial_{1}+1\right) v-\left(x_{1}+1\right) w_{2} .
\end{array}\right.
\end{aligned}
$$

Now, since $M$ is torsion-free (see, e.g., [11]), so is $O$. Then, computing a minimal parametrization of $O$ (see [11]), $O$ is isomorphic to the left ideal $I=D \partial_{1}^{2}+D\left(\left(x_{1}+1\right) \partial_{1} \partial_{3}-\partial_{1} \partial_{2}-\partial_{3}\right)$ generated by two elements of $D$, which finally shows that $M \cong D \oplus I$. 
Example 6. Let $M=D^{1 \times 6} /\left(D^{1 \times 3} R\right)$ be the $D=\mathbb{Q}\left[\partial_{1}, \partial_{2}\right]$-module finitely presented by

$$
R=\left(\begin{array}{cccccc}
\partial_{1} & \partial_{2} & 0 & 0 & 0 & 0 \\
0 & -1 & 1 & 0 & \partial_{1} & \partial_{2} \\
0 & 0 & \partial_{1} & \partial_{2} & 0 & 0
\end{array}\right)
$$

which corresponds to the following Cosserat's equations

$$
R\left(\begin{array}{c}
\sigma^{11} \\
\sigma^{12} \\
\sigma^{21} \\
\sigma^{22} \\
\mu^{1} \\
\mu^{2}
\end{array}\right)=0 \Leftrightarrow\left\{\begin{array}{l}
\partial_{1} \sigma^{11}+\partial_{2} \sigma^{12}=0 \\
\partial_{1} \mu^{1}+\partial_{2} \mu^{2}+\sigma^{21}-\sigma^{12}=0 \\
\partial_{1} \sigma^{21}+\partial_{2} \sigma^{22}=0
\end{array}\right.
$$

where $\left(\sigma^{11}, \sigma^{12}, \sigma^{21}, \sigma^{22}\right)$ denotes the possibly non-symmetric stress tensor and $\left(\mu^{1}, \mu^{2}\right)$ a couple-stress tensor. If the couple-stress tensor vanishes, i.e., $\mu^{1}=\mu^{2}=0$, then (21) yields the classical equation for the equilibrium of the symmetric stress tensor (i.e., $\bar{\sigma}^{12}=\bar{\sigma}^{21}$ ):

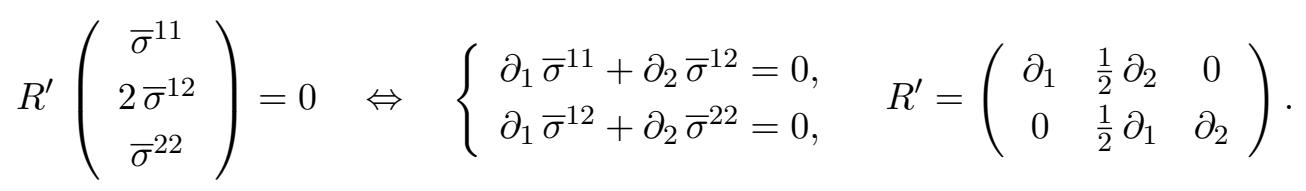

If $\bar{R}=\left(\begin{array}{ll}0 & R^{\prime}\end{array}\right) \in D^{2 \times 5}$, then using the above constructive techniques, we can prove that

$$
\begin{aligned}
& f: M \longrightarrow \bar{M}:=D^{1 \times 5} /\left(D^{1 \times 2} \bar{R}\right) \quad g: \bar{M} \longrightarrow M \\
& \pi(\lambda) \longrightarrow \bar{\pi}\left(\lambda P_{1}\right), \quad \bar{\pi}(\theta) \longrightarrow \pi\left(\theta P_{1}^{\prime}\right),
\end{aligned}
$$

are $D$-isomorphisms and $f=g^{-1}$, where $\pi: D^{1 \times 6} \longrightarrow M\left(\right.$ resp., $\bar{\pi}: D^{1 \times 5} \longrightarrow \bar{M}$ ) is the canonical projection onto $M$ (resp., $\bar{M}$ ) and:

$$
P_{1}=\left(\begin{array}{ccccc}
\partial_{2} & 0 & 1 & 0 & 0 \\
-\partial_{1} & 0 & 0 & \frac{1}{2} & 0 \\
0 & \partial_{2} & 0 & \frac{1}{2} & 0 \\
0 & -\partial_{1} & 0 & 0 & 1 \\
-1 & 0 & 0 & 0 & 0 \\
0 & -1 & 0 & 0 & 0
\end{array}\right), \quad P_{1}^{\prime}=\left(\begin{array}{cccccc}
0 & 0 & 0 & 0 & -1 & 0 \\
0 & 0 & 0 & 0 & 0 & -1 \\
1 & 0 & 0 & 0 & \partial_{2} & 0 \\
0 & 0 & 2 & 0 & 0 & 2 \partial_{2} \\
0 & 0 & 0 & 1 & 0 & -\partial_{1}
\end{array}\right)
$$

Hence, we obtain that $M \cong D \mu^{1} \oplus D \mu^{2} \oplus M^{\prime}$, where $M^{\prime}:=D^{1 \times 3} /\left(D^{1 \times 2} R^{\prime}\right)$ corresponds to the linear PD system (22), i.e., $M \cong D^{1 \times 2} \oplus M^{\prime}$, where $\operatorname{rank}_{D}\left(M^{\prime}\right)=1$. 
If $\mathcal{F}$ is a $D$-module (i.e., $\mathcal{F}=C^{\infty}\left(\mathbb{R}^{2}\right)$ ), then $\operatorname{ker}_{\mathcal{F}}(\bar{R}$. $)=\mathcal{F}^{2} \oplus \operatorname{ker}_{\mathcal{F}}\left(R^{\prime}\right.$.) $\cong \operatorname{ker}_{\mathcal{F}}(R$.) and the $D$-isomorphisms $f$ and $g=f^{-1}$ show that the following equivalences of systems hold:

$$
\begin{gathered}
\operatorname{ker}_{\mathcal{F}}(R .) \stackrel{g^{\star}}{\longrightarrow} \operatorname{ker}_{\mathcal{F}}(\bar{R} .) \\
\eta=\left(\begin{array}{l}
\sigma^{11} \\
\sigma^{12} \\
\sigma^{21} \\
\sigma^{22} \\
\mu^{1} \\
\mu^{2}
\end{array}\right) \\
\end{gathered}
$$

$\operatorname{ker}_{\mathcal{F}}(\bar{R}.) \stackrel{f^{\star}}{\longrightarrow} \operatorname{ker}_{\mathcal{F}}(R$.

$$
\bar{\eta}=\left(\begin{array}{c}
\bar{\mu}^{1} \\
\bar{\mu}^{2} \\
\bar{\sigma}^{11} \\
2 \bar{\sigma}^{12} \\
\bar{\sigma}^{22}
\end{array}\right) \longmapsto P_{1} \bar{\eta}=\left(\begin{array}{l}
\sigma^{11}=\bar{\sigma}^{11}+\partial_{2} \bar{\mu}^{1} \\
\sigma^{12}=\bar{\sigma}^{12}-\partial_{1} \bar{\mu}^{1} \\
\sigma^{21}=\bar{\sigma}^{12}+\partial_{2} \bar{\mu}^{2} \\
\sigma^{22}=\bar{\sigma}^{22}-\partial_{1} \bar{\mu}^{2} \\
\mu^{1}=-\bar{\mu}^{1} \\
\mu^{2}=-\bar{\mu}^{2}
\end{array}\right) .
$$

Using $g^{\star}$ and the canonical projection $\operatorname{ker}_{\mathcal{F}}(\bar{R}$. $) \longrightarrow \operatorname{ker}_{\mathcal{F}}\left(R^{\prime}\right.$.), if $\eta \in \operatorname{ker}_{\mathcal{F}}(R$. $)$, then:

$$
\left(\bar{\sigma}^{11}=\sigma^{11}+\partial_{2} \mu^{1}, 2 \bar{\sigma}^{12}=2\left(\sigma^{21}+\partial_{2} \mu^{2}\right), \bar{\sigma}^{22}=\sigma^{22}-\partial_{1} \mu^{2}\right)^{T} \in \operatorname{ker}_{\mathcal{F}}\left(R^{\prime} .\right) .
$$

In particular, this last map $\vartheta: \operatorname{ker}_{\mathcal{F}}(R.) \longrightarrow \operatorname{ker}_{\mathcal{F}}\left(R^{\prime}\right.$.) is surjective and $\operatorname{ker} \vartheta \cong \mathcal{F}^{2}$.

More explicitly, if we consider $\bar{P}=\left(\begin{array}{ll}0 & I_{3}\end{array}\right) P_{1}^{\prime} \in D^{3 \times 6}, T=\left(\begin{array}{ll}0 & -I_{2}\end{array}\right) \in D^{2 \times 6}$,

$$
Q=\left(\begin{array}{cccccc}
\partial_{2} & -\partial_{1} & 0 & 0 & -1 & 0 \\
0 & 0 & \partial_{2} & -\partial_{1} & 0 & -1
\end{array}\right)^{T}, \quad S=\left(\begin{array}{cccccc}
1 & 0 & 0 & 0 & 0 & 0 \\
0 & 1 / 2 & 1 / 2 & 0 & 0 & 0 \\
0 & 0 & 0 & 1 & 0 & 0
\end{array}\right)^{T}
$$

then the following extension of $D^{1 \times 2}$ by $M^{\prime}$, i.e., the following short exact sequence

$$
0 \longrightarrow M^{\prime} \stackrel{\iota}{\longrightarrow} M \stackrel{\omega}{\longrightarrow} D^{1 \times 2} \longrightarrow 0,
$$

where $i \in \operatorname{hom}_{D}\left(M^{\prime}, M\right)$ is defined by $i\left(\pi^{\prime}\left(\lambda^{\prime}\right)\right)=\pi\left(\lambda^{\prime} \bar{P}\right)$ for all $\lambda^{\prime} \in D^{1 \times 3}, \pi^{\prime}: D^{1 \times 3} \longrightarrow M^{\prime}$ is the canonical projection onto $M^{\prime}$, and $\omega(\pi(\lambda))=\lambda Q$ for all $\lambda=\left(\lambda_{1} \ldots \lambda_{6}\right) \in D^{1 \times 6}$, is trivial, namely, (25) is a split short exact sequence. In particular, $\tau: D^{1 \times 2} \longrightarrow M$ defined by $\tau(\theta)=\pi(\theta T)$ for all $\theta \in D^{1 \times 2}$ satisfies $\omega \circ \tau=\operatorname{id}_{D^{1 \times 2}}$, and $\psi: M \longrightarrow M^{\prime}$ defined by $\psi(\pi(\lambda))=\pi^{\prime}(\lambda S)$ for all $\lambda \in D^{1 \times 6}$ satisfies $\psi \circ i=\operatorname{id}_{M^{\prime}}$. 
Finally, applying the contravariant left exact functor $\operatorname{hom}_{D}(\cdot, \mathcal{F})$ (see, e.g., [38]) to the split exact sequence (25) and using (2), we get the split exact sequence (see, e.g., [38])

$$
0 \longleftarrow \operatorname{ker}_{\mathcal{F}}\left(R^{\prime} .\right) \stackrel{\iota^{\star}}{\longleftarrow} \operatorname{ker}_{\mathcal{F}}(R .) \stackrel{Q .}{\longleftarrow} \mathcal{F}^{2} \longleftarrow 0,
$$

where $\iota^{\star}$ is defined by (24), which shows that $\operatorname{ker}_{\mathcal{F}}(R)=.S \operatorname{ker}_{\mathcal{F}}\left(R^{\prime}\right.$.) $\oplus Q \mathcal{F}^{2}$ (see also (23)).

Remark 5. Let us give system-theoretical applications of Theorem 7. Let $M$ be a finitely generated left $D$-module with $\operatorname{rank}_{D}(M) \geq 2$. Then, according to Theorem 7 , there exist $r \in \mathbb{Z}_{\geq 0}$ and a left $D$-module $M^{\prime}$ with $\operatorname{rank}_{D}\left(M^{\prime}\right) \leq 1$ such that $M \cong D^{1 \times r} \oplus M^{\prime}$. If $\mathcal{F}$ is a left $D$-module, then using the additivity of the functor $\operatorname{hom}_{D}(\cdot, \mathcal{F})$ (see, e.g., [38]), we get:

$$
\operatorname{hom}_{D}(M, \mathcal{F}) \cong \operatorname{hom}_{D}\left(D^{1 \times r}, \mathcal{F}\right) \oplus \operatorname{hom}_{D}\left(M^{\prime}, \mathcal{F}\right) \cong \mathcal{F}^{r} \oplus \operatorname{hom}_{D}\left(M^{\prime}, \mathcal{F}\right)
$$

Significant information on the solution space $\operatorname{hom}_{D}(M, \mathcal{F})$ is then contained in $\operatorname{hom}_{D}\left(M^{\prime}, \mathcal{F}\right)$. We refer to [11] for the relevance of the extension functor $\operatorname{ext}_{D}^{i}(\cdot, \mathcal{F})$ in mathematical systems theory. More generally, since $\operatorname{ext}_{D}^{i}\left(D^{1 \times r}, \mathcal{F}\right)=0$ for $i \geq 1$ (see, e.g., [38]), we then have:

$$
\forall i \geq 1, \quad \operatorname{ext}_{D}^{i}(M, \mathcal{F}) \cong \operatorname{ext}_{D}^{i}\left(M^{\prime}, \mathcal{F}\right)
$$

A more general version of Theorem 7 , namely, a relative version where $M$ is replaced by $M \subseteq N$ or, more generally, by an injective homomorphism $\iota: M \longrightarrow N$, is now given.

Theorem 8 ([42]). Let $M$ and $N$ be two finitely generated left $D$-modules satisfying $M \subseteq N$ and $\operatorname{rank}_{D}(M) \geq 2$. Then, there exists $m^{\star} \in M$ which is a unimodular element of $N$. Hence, there exist a left $D$-submodule $M^{\prime}$ (resp., $\left.N^{\prime}\right)$ of $M$ (resp., $\left.N\right)$ such that $M^{\prime}=M \cap N^{\prime}$ and:

$$
M=D m^{\star} \oplus M^{\prime} \subseteq N=D m^{\star} \oplus N^{\prime} .
$$

Proof. Let us consider a presentation of the finitely generated left $D$-module $M$ (resp., $N$ ) $M=D^{1 \times p} /\left(D^{1 \times q} R\right)$ (resp., $\left.N=D^{1 \times p^{\prime}} /\left(D^{1 \times q^{\prime}} R^{\prime}\right)\right)$, where $R \in D^{q \times p}$ (resp., $\left.R^{\prime} \in D^{q^{\prime} \times p^{\prime}}\right)$. Let $\iota \in \operatorname{hom}_{D}(M, N)$ be the injection of $M$ into $N$. Firstly, $\iota \in \operatorname{hom}_{D}(M, N)$ is defined by the following commutative diagram with exact rows

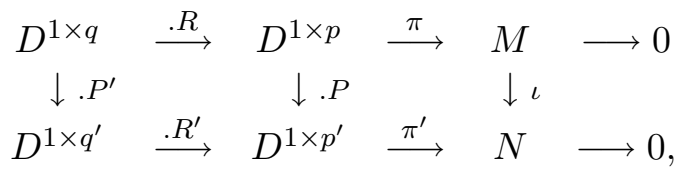

i.e., $\iota(\pi(\eta))=\pi^{\prime}(\eta P)$ for all $\eta \in D^{1 \times p}$, where $P \in D^{p \times p^{\prime}}$ is such that $R P=P^{\prime} R^{\prime}$ for a certain $P^{\prime} \in D^{q \times q^{\prime}}$ and $\pi^{\prime}: D^{1 \times p^{\prime}} \longrightarrow N$ is the canonical projection onto $N$ (see [13]). Secondly, the injectivity of $\iota$ is equivalent to the fact that for all $S \in D^{s \times p}$ and for all $T \in D^{s \times q^{\prime}}$ satisfying $S P=T R^{\prime}$, there exists $L \in D^{s \times q}$ such that $S=L R$. For more details, see [13]. Finally, we have $\iota(M)=\left(D^{1 \times\left(p+q^{\prime}\right)}\left(P^{T} \quad R^{\prime T}\right)^{T}\right) /\left(D^{1 \times q^{\prime}} R^{\prime}\right) \subseteq N=D^{1 \times p^{\prime}} /\left(D^{1 \times q^{\prime}} R^{\prime}\right)$ (see [13]).

RR n ${ }^{\circ} 8225$ 
Theorem 8 follows similar arguments as the ones used in the proof of Theorem 7 . Let us shortly adapt the arguments of the proof of Theorem 7 to this more general situation. We note that Theorem 7 is a particular case of Theorem 8 where $N=M$ and $\iota=\mathrm{id}_{M}$.

The hypothesis $\operatorname{rank}_{D}(M) \geq 2$ implies that $M$ is not torsion by $1 \Rightarrow 3$ of Lemma 1 . Then, there exists $m_{1}=\pi\left(\eta_{1}\right) \notin t(M)$. Thus, if $\lambda_{1}=\eta_{1} P$, then $\iota\left(m_{1}\right)=\pi^{\prime}\left(\lambda_{1}\right) \notin t(N)$. If $Q \in D^{p \times m}$ (resp., $Q^{\prime} \in D^{p^{\prime} \times m^{\prime}}$ ) is such that $\operatorname{ker}_{D}(R$. $)=Q D^{m}\left(\operatorname{resp} ., \operatorname{ker}_{D}\left(R^{\prime}\right.\right.$.) $=Q^{\prime} D^{m^{\prime}}$ ), then $\lambda_{1} \in D^{1 \times p}$ has to be chosen so that $\lambda_{1} Q \neq 0$ (or such that $\lambda_{1} P Q^{\prime} \neq 0$ ). Now, Remark 1 shows that there exists $\mu_{1} \in \operatorname{ker}_{D}\left(R^{\prime}\right.$. $)=Q^{\prime} D^{m^{\prime}}$ such that $\varphi_{1}:=\varphi_{\mu_{1}}$ satisfies $\varphi_{1}\left(\iota\left(m_{1}\right)\right) \neq 0$. More precisely, we have to choose $\xi_{1} \in D^{m^{\prime}}$ such that $\mu_{1}=Q^{\prime} \xi_{1}$ satisfies:

$$
\varphi_{1}\left(\iota\left(m_{1}\right)\right)=\lambda_{1} \mu_{1}=\left(\lambda_{1} Q^{\prime}\right) \xi_{1} \neq 0 .
$$

Then, the following diagram is commutative with exact rows:

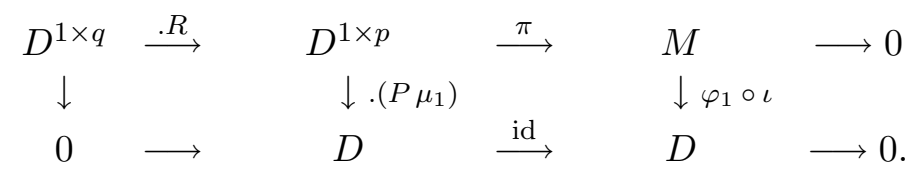

Since $\operatorname{im}\left(\varphi_{1} \circ \iota\right)$ is a left ideal of $D$ containing $\left(\varphi_{1} \circ \iota\right)\left(m_{1}\right) \neq 0, \operatorname{rank}_{D}\left(\operatorname{im}\left(\varphi_{1} \circ \iota\right)\right)=1$, which combined with the short exact sequence $0 \longrightarrow \operatorname{ker}\left(\varphi_{1} \circ \iota\right) \longrightarrow M \longrightarrow \operatorname{im}\left(\varphi_{1} \circ \iota\right) \longrightarrow 0$ yields $\operatorname{rank}_{D}\left(\operatorname{ker}\left(\varphi_{1} \circ \iota\right)\right)=\operatorname{rank}_{D}(M)-1 \geq 1$. Thus, $\operatorname{ker}\left(\varphi_{1} \circ \iota\right)$ is not torsion by $1 \Rightarrow 3$ of Lemma 1. Since $t\left(\operatorname{ker}\left(\varphi_{1} \circ \iota\right)\right)=t(M) \cap \operatorname{ker}\left(\varphi_{1} \circ \iota\right)$, there exists $m_{2} \in \operatorname{ker}\left(\varphi_{1} \circ \iota\right)$ such that $m_{2} \notin t(M)$, and thus $\iota\left(m_{2}\right) \notin t(N)$. Let $S \in D^{r \times p}$ be such that $\operatorname{ker}_{D}\left(.\left(P \mu_{1}\right)\right)=D^{1 \times r} S$. Then, we have

$$
\operatorname{ker}\left(\varphi_{1} \circ \iota\right)=\operatorname{ker}_{D}\left(.\left(P \mu_{1}\right)\right) /\left(D^{1 \times q} R\right)=\left(D^{1 \times r} S\right) /\left(D^{1 \times q} R\right),
$$

and thus $m_{2}=\pi\left(\eta_{2}\right)$ for some $\eta_{2}=\nu S \in D^{1 \times p}$, where $\nu \in D^{1 \times r}$ has to be chosen so that $\nu\left(S P Q^{\prime}\right) \neq 0$. Define $\lambda_{2}=\eta_{2} P$. Considering $\xi_{2} \in D^{m^{\prime}}$ such that $\left(\lambda_{2} Q^{\prime}\right) \xi_{2} \neq 0$ and $\mu_{2}:=Q^{\prime} \xi_{2} \in D^{p}$, then $\varphi_{2}:=\varphi_{\mu_{2}}$ satisfies $\varphi_{2}\left(\iota\left(m_{2}\right)\right)=\lambda_{2} \mu_{2} \neq 0$.

By construction, $m_{2} \in \operatorname{ker}\left(\varphi_{1} \circ \iota\right)$, which yields $\varphi_{1}\left(\iota\left(m_{2}\right)\right)=\lambda_{2} \mu_{1}=0$. Now, if $\varphi_{2}\left(\iota\left(m_{1}\right)\right)=\lambda_{1} \mu_{2} \neq 0$, then by the right Ore property of $D$, there exist $r_{1}, r_{2} \in D \backslash\{0\}$ such that $\left(\lambda_{1} \mu_{1}\right) r_{1}+\left(\lambda_{1} \mu_{2}\right) r_{2}=0$. Let $\mu_{2}^{\prime}:=\mu_{1} r_{1}+\mu_{2} r_{2} \in \operatorname{ker}_{D}\left(R^{\prime}\right.$.) and define $\varphi_{2}^{\prime}:=\varphi_{\mu_{1}} r_{1}+\varphi_{\mu_{2}} r_{2}=\varphi_{\mu_{2}^{\prime}} \in \operatorname{hom}_{D}(N, D)$. Then, we have:

$$
\left\{\begin{array}{l}
\varphi_{2}^{\prime}\left(\iota\left(m_{1}\right)\right)=\lambda_{1} \mu_{2}^{\prime}=\lambda_{1}\left(\mu_{1} r_{1}+\mu_{2} r_{2}\right)=0, \\
\varphi_{2}^{\prime}\left(\iota\left(m_{2}\right)\right)=\lambda_{2} \mu_{2}^{\prime}=\lambda_{2}\left(\mu_{1} r_{1}+\mu_{2} r_{2}\right)=\left(\lambda_{2} \mu_{2}\right) r_{2} \neq 0 .
\end{array}\right.
$$

Therefore, without loss of generality, we may assume that $\varphi_{2}\left(\iota\left(m_{1}\right)\right)=0$.

Let $d_{1}:=\lambda_{1} \mu_{1} \neq 0$ and $d_{2}:=\lambda_{2} \mu_{2} \neq 0$. Then, Corollary 1 shows that there exists $\left(y_{1} y_{2} z_{1} z_{2}\right)^{T} \in D^{4}$ satisfying $y_{1}\left(\lambda_{1} \mu_{1}\right) z_{1}+y_{2}\left(\lambda_{2} \mu_{2}\right) z_{2}=1$. If we define

$$
\begin{array}{ll}
\eta^{\star}:=y_{1} \eta_{1}+y_{2} \eta_{2}, & m^{\star}:=\pi\left(\eta^{\star}\right)=y_{1} \pi\left(\eta_{1}\right)+y_{2} \pi\left(\eta_{2}\right) \in M, \\
\mu^{\star}:=\mu_{1} z_{1}+\mu_{2} z_{2} \in \operatorname{ker}_{D}\left(R^{\prime} .\right), & \varphi:=\varphi_{\mu^{\star}} \in \operatorname{hom}_{D}(N, D),
\end{array}
$$

$\mathrm{RR} \mathrm{n}^{\circ} 8225$ 
then we have:

$$
\begin{aligned}
\varphi\left(\iota\left(m^{\star}\right)\right) & =\eta^{\star} P \mu^{\star}=\left(y_{1} \eta_{1}+y_{2} \eta_{2}\right) P\left(\mu_{1} z_{1}+\mu_{2} z_{2}\right)=\left(y_{1} \lambda_{1}+y_{2} \lambda_{2}\right)\left(\mu_{1} z_{1}+\mu_{2} z_{2}\right) \\
& =y_{1}\left(\lambda_{1} \mu_{1}\right) z_{1}+y_{1}\left(\lambda_{1} \mu_{2}\right) z_{2}+y_{2}\left(\lambda_{2} \mu_{1}\right) z_{1}+y_{2}\left(\lambda_{2} \mu_{2}\right) z_{2} \\
& =y_{1}\left(\lambda_{1} \mu_{1}\right) z_{1}+y_{2}\left(\lambda_{2} \mu_{2}\right) z_{2}=1 .
\end{aligned}
$$

Thus, $\iota\left(m^{\star}\right) \in \iota(M)$ is a unimodular element of $N$, which yields $N=D \iota\left(m^{\star}\right) \oplus \operatorname{ker} \varphi$. Moreover, since $\psi:=\varphi_{\mid \iota(M)} \in \operatorname{hom}_{D}(\iota(M), D)$ satisfies $\psi\left(\iota\left(m^{\star}\right)\right)=1, \iota\left(m^{\star}\right)$ is also a unimodular element of $\iota(M)$, which shows that $\iota(M)=D \iota\left(m^{\star}\right) \oplus \operatorname{ker} \psi$, and finally proves

$$
\iota(M)=D \iota\left(m^{\star}\right) \oplus M^{\prime} \subseteq N=D \iota\left(m^{\star}\right) \oplus N^{\prime},
$$

where $N^{\prime}:=\operatorname{ker} \varphi$ and $M^{\prime}:=\operatorname{ker} \varphi_{\mid \iota(M)}=\operatorname{ker} \varphi \cap \iota(M)$.

Remark 6. We note that a division ring $D$ is characterized by the result of Theorem 8 for finitely generated left $D$-modules $M \subseteq N$ if the condition $\operatorname{rank}_{D}(M) \geq 2$ is dropped. Indeed, Theorem 8 holds for nonzero finitely generated $D$-vector spaces $M \subseteq N$.

A unimodular element, whose existence is proved in Theorem 8 , can be computed by the following algorithm.

Algorithm 2 (UnimodularElementInSubmodule).

- Input: A very simple domain $D$ and $R \in D^{q \times p}$ such that the finitely presented left $D$-module $M=D^{1 \times p} /\left(D^{1 \times q} R\right)$ has rank at least two, i.e., $\operatorname{rank}_{D}(M) \geq 2$, a matrix $R^{\prime} \in D^{q^{\prime} \times p^{\prime}}$ presenting a left $D$-module $N=D^{1 \times p^{\prime}} /\left(D^{1 \times q^{\prime}} R^{\prime}\right)$, and a matrix $P \in D^{p \times p^{\prime}}$ defining an injective homomorphism $\iota \in \operatorname{hom}_{D}(M, N)$, i.e., $\iota(\pi(\eta))=\pi^{\prime}(\eta P)$ for all $\eta \in D^{1 \times p}$, where $\pi$ (resp., $\pi^{\prime}$ ) is the canonical projection onto $M($ resp., $N)$.

- Output: $\eta^{\star} \in D^{1 \times p}$ and $\mu^{\star} \in \operatorname{ker}_{D}\left(R^{\prime}\right.$. $)$ such that $\iota\left(\pi\left(\eta^{\star}\right)\right)=\pi^{\prime}\left(\eta^{\star} P\right) \in \mathrm{U}(N)$ and $\varphi_{\mu^{\star}} \in \operatorname{hom}_{D}(N, D)$ such that $\varphi_{\mu^{\star}}\left(\iota\left(\pi\left(\eta^{\star}\right)\right)\right)=1$.

1. Compute $Q^{\prime} \in D^{p^{\prime} \times m^{\prime}}$ such that $\operatorname{ker}_{D}\left(R^{\prime}.\right)=Q^{\prime} D^{m^{\prime}}$.

2. Pick $\eta_{1} \in D^{1 \times p}$ such that $\eta_{1}\left(P Q^{\prime}\right) \neq 0$ and define $\lambda_{1}=\eta_{1} P$.

3. Find $\xi_{1} \in D^{m^{\prime}}$ such that $\left(\lambda_{1} Q^{\prime}\right) \xi_{1} \neq 0$ and define $\mu_{1}=Q^{\prime} \xi_{1} \in \operatorname{ker}_{D}\left(R^{\prime}\right.$.).

4. Compute $S \in D^{r \times p}$ such that $\operatorname{ker}_{D}\left(.\left(P \mu_{1}\right)\right)=D^{1 \times r} S$.

5. Pick $\nu \in D^{1 \times r}$ such that $\nu\left(S P Q^{\prime}\right) \neq 0$ and define $\eta_{2}=\nu S$ and $\lambda_{2}=\eta_{2} P$.

6. Find $\xi_{2} \in D^{m^{\prime}}$ such that $\left(\lambda_{2} Q^{\prime}\right) \xi_{2} \neq 0$ and define $\mu_{2}=Q^{\prime} \xi_{2} \in \operatorname{ker}_{D}\left(R^{\prime}\right.$.).

7. If $\lambda_{1} \mu_{2} \neq 0$, then compute $r_{1}, r_{2} \in D \backslash\{0\}$ such that $\left(\lambda_{1} \mu_{1}\right) r_{1}+\left(\lambda_{1} \mu_{2}\right) r_{2}=0$ and replace $\mu_{2}$ by $\mu_{1} r_{1}+\mu_{2} r_{2}$. 
8. Compute $y_{1}, y_{2}, z_{1}, z_{2} \in D$ such that $y_{1}\left(\lambda_{1} \mu_{1}\right) z_{1}+y_{2}\left(\lambda_{2} \mu_{2}\right) z_{2}=1$.

9. Return $\eta^{\star}=y_{1} \eta_{1}+y_{2} \eta_{2} \in D^{1 \times p}$ and $\mu^{\star}=\mu_{1} z_{1}+\mu_{2} z_{2} \in \operatorname{ker}_{D}\left(R^{\prime}\right.$.).

Example 7. Let $D=A_{1}(\mathbb{Q})$ and $M=D^{1 \times 3} /(D R)$ and $N=D^{1 \times 6} /\left(D^{1 \times 3} R^{\prime}\right)$ be left $D$ modules finitely presented respectively by:

$$
R=\left(\begin{array}{lll}
\partial & 0 & -t
\end{array}\right), \quad R^{\prime}=\left(\begin{array}{cccccc}
\partial & -t & 0 & 0 & 0 & -1 \\
0 & \partial & 0 & -t & 0 & 0 \\
0 & 0 & \partial & 0 & -t & 0
\end{array}\right) .
$$

Since $R$ and $R^{\prime}$ have full row rank, we have $\operatorname{rank}_{D}(M)=2$ and $\operatorname{rank}_{D}(N)=3$. Now, if

$$
P=\left(\begin{array}{llllll}
0 & 0 & 1 & 0 & 0 & 0 \\
1 & 0 & 0 & 0 & 0 & 1 \\
0 & 0 & 0 & 0 & 1 & 0
\end{array}\right), \quad P^{\prime}=\left(\begin{array}{lll}
0 & 0 & 1
\end{array}\right),
$$

then we have $R P=P^{\prime} R^{\prime}$, i.e., the diagram (28) is commutative with exact rows, where $\iota \in \operatorname{hom}_{D}(M, N)$ is defined by $\iota(\pi(\eta))=\pi^{\prime}(\eta P)$ for all $\eta \in D^{1 \times 3}$, and $\pi: D^{1 \times 3} \longrightarrow M$ (resp., $\pi^{\prime}: D^{1 \times 6} \longrightarrow N$ ) is the canonical projection onto $M$ (resp., $N$ ). Then, $\iota$ is injective because:

$$
\operatorname{ker}_{D}\left(.\left(P^{T} \quad R^{\prime T}\right)^{T}\right)=D\left(\begin{array}{ll}
S & -T
\end{array}\right), \quad S=\left(\begin{array}{lll}
\partial & 0 & -t
\end{array}\right)=R, \quad T=\left(\begin{array}{lll}
0 & 0 & 1
\end{array}\right) .
$$

We can check that $\operatorname{ker}_{D}\left(R^{\prime}\right.$. $)=Q^{\prime} D^{5}$, where:

$$
Q^{\prime}=\left(\begin{array}{ccccc}
-1 & t^{2} & 0 & 0 & 0 \\
0 & t \partial-1 & -t^{2} & 0 & 0 \\
0 & 0 & 0 & -t^{2} & t \partial-1 \\
0 & \partial^{2} & -t \partial-2 & 0 & 0 \\
0 & 0 & 0 & -t \partial-2 & \partial^{2} \\
-\partial & 3 t & t^{3} & 0 & 0
\end{array}\right)
$$

If $\eta_{1}=\left(\begin{array}{lll}0 & 1 & 0\end{array}\right)$ and $\xi_{1}=\left(\begin{array}{lllll}-1 & 0 & 0 & 0 & 0\end{array}\right)^{T}$, then $\mu_{1}=Q^{\prime} \xi_{1}=\left(\begin{array}{llllll}1 & 0 & 0 & 0 & 0 & \partial\end{array}\right)^{T}$ and $d_{1}=\eta_{1} P \mu_{1}=\partial+1$. Now, $\operatorname{ker}_{D}\left(.\left(P \mu_{1}\right)\right)=D^{1 \times 2} S$, where:

$$
S=\left(\begin{array}{lll}
1 & 0 & 0 \\
0 & 0 & 1
\end{array}\right)
$$

If $\nu=\left(\begin{array}{ll}0 & 1\end{array}\right)$ and $\xi_{2}=\left(\begin{array}{lllll}0 & 0 & 0 & 0 & 1\end{array}\right)^{T}$, then $\eta_{2}=\nu S=\left(\begin{array}{lll}0 & 0 & 1\end{array}\right), \mu_{2}=Q^{\prime} \xi_{2}=$ $\left(\begin{array}{llllll}0 & 0 & t \partial-1 & 0 & \partial^{2} & 0\end{array}\right)^{T}, d_{2}=\eta_{2} P \mu_{2}=\partial^{2}$, and $\eta_{1} P \mu_{2}=0$. Then, $z_{1}=1, z_{2}=t+1$, $y_{1}=-(t+1) \partial^{2}-3 \partial+1$, and $y_{2}=\partial+1$ are such that $y_{1} d_{1} z_{1}+y_{2} d_{2} z_{2}=1$, which yields

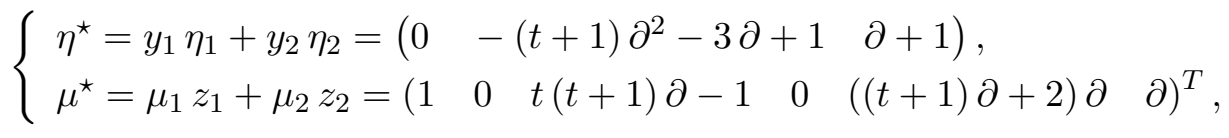

$\mathrm{RR} \mathrm{n}^{\circ} 8225$ 
which satisfy $\eta^{\star} P \mu^{\star}=1$. Thus, $m^{\prime}=\pi^{\prime}\left(\eta^{\star} P\right) \in \mathrm{U}(N)$ because $\varphi\left(m^{\prime}\right)=1$, where $\varphi:=\varphi_{\mu^{\star}}$. Moreover, if $m^{\star}=\pi\left(\eta^{\star}\right) \in M$, then $\varphi_{\mid \iota(M)}\left(m^{\star}\right)=1$, i.e., $\iota\left(m^{\star}\right)=\pi^{\prime}\left(\eta^{\star} P\right)=m^{\prime} \in \mathrm{U}(\iota(M))$, which yields $\iota(M)=D m^{\prime} \oplus M^{\prime} \subseteq N=D m^{\prime} \oplus N^{\prime}$, where $N^{\prime}=\operatorname{ker} \varphi$ and $M^{\prime}=\operatorname{ker} \varphi_{\mid \iota(M)}$.

\section{Stafford's reduction}

Let us give now an important application of Theorem 8 and Algorithm 2. Let us consider a finitely presented left $D$-module $L=D^{1 \times p^{\prime}} /\left(D^{1 \times p} P\right)$ and the left $D$-modules $K=D^{1 \times p} P$ and $N=D^{1 \times p^{\prime}}$. Let $R \in D^{q \times p}$ (possibly $R=0$ and $q=0$ ) be such that $\operatorname{ker}_{D}(. P)=D^{1 \times q} R$. Then, $M:=D^{1 \times p} /\left(D^{1 \times q} R\right) \cong K$ and the injection $\iota: M \longrightarrow D^{1 \times p^{\prime}}$ is defined by

$$
\forall \eta \in D^{1 \times p}, \quad \iota(\pi(\eta))=\eta P,
$$

where $\pi: D^{1 \times p} \longrightarrow M$ is the canonical projection onto $M$. Note that $K=\iota(M)$. The following diagram is commutative with exact rows:

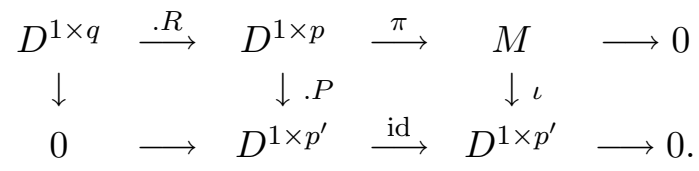

Now, if $\operatorname{rank}_{D}(K)=\operatorname{rank}_{D}(M) \geq 2$, then Theorem 8 is applicable. Since $\operatorname{hom}_{D}(N, D) \cong D^{p^{\prime}}$, we can take $Q^{\prime}=I_{p^{\prime}}$ in Algorithm 2 and we obtain $\eta^{\star} \in D^{1 \times p}$ and $\xi^{\star} \in D^{p^{\prime}}$ such that $m^{\star}=\pi\left(\eta^{\star}\right) \in M$ satisfies that $\iota\left(m^{\star}\right)=\eta^{\star} P \in \mathrm{U}\left(D^{1 \times p^{\prime}}\right)$ and $\varphi:=\varphi_{\xi^{\star}}$ satisfies $\varphi\left(\iota\left(m^{\star}\right)\right)=$ $\eta^{\star} P \xi^{\star}=1$. If $\lambda^{\star}:=\eta^{\star} P \in D^{1 \times p^{\prime}}$, then we get:

$$
D^{1 \times p^{\prime}}=D \iota\left(m^{\star}\right) \oplus \operatorname{ker} \varphi=D \lambda^{\star} \oplus \operatorname{ker} \varphi .
$$

Since $\iota\left(m^{\star}\right)=\lambda^{\star}$ is also a unimodular element of $K=\iota(M)$, we get

$$
K=D^{1 \times p} P=D \iota\left(m^{\star}\right) \oplus \operatorname{ker} \varphi_{\mid K}=D \lambda^{\star} \oplus \operatorname{ker} \varphi_{\mid K},
$$

where $\operatorname{ker} \varphi_{\mid K}=\operatorname{ker} \varphi \cap K$. Hence, we obtain

$$
L=D^{1 \times p^{\prime}} /\left(D^{1 \times p} P\right)=\left(D \lambda^{\star} \oplus \operatorname{ker} \varphi\right) /\left(D \lambda^{\star} \oplus \operatorname{ker} \varphi_{\mid K}\right) \cong \operatorname{ker} \varphi / \operatorname{ker} \varphi_{\mid K},
$$

where $\operatorname{rank}_{D}(\operatorname{ker} \varphi)=p^{\prime}-1$ and $\operatorname{rank}_{D}\left(\operatorname{ker} \varphi_{\mid K}\right)=\operatorname{rank}_{D}(K)-1$.

Let us now characterize the left $D$-module $L^{\prime}:=\operatorname{ker} \varphi / \operatorname{ker} \varphi_{\mid K}$ and the isomorphism $L \cong L^{\prime}$ appearing in (29). We have $\operatorname{ker} \varphi=\operatorname{ker}_{D}\left(. \xi^{\star}\right)=D^{1 \times r} X$ for a certain $X \in D^{r \times p^{\prime}}$, and $\operatorname{ker} \varphi_{\mid K}=\left\{\eta P \mid \eta \in D^{1 \times p}:(\eta P) \xi^{\star}=0\right\}=\operatorname{ker}_{D}\left(.\left(P \xi^{\star}\right)\right) P$. If $Y \in D^{s \times p}$ is such that $\operatorname{ker}_{D}\left(.\left(P \xi^{\star}\right)\right)=D^{1 \times s} Y$ and $Z=Y P \in D^{s \times p^{\prime}}$, then $\operatorname{ker} \varphi_{\mid K}=D^{1 \times s} Z$, which yields:

$$
L^{\prime}=\left(D^{1 \times r} X\right) /\left(D^{1 \times s} Z\right)
$$

$\mathrm{RR} \mathrm{n}^{\circ} 8225$ 
We note that $\operatorname{rank}_{D}\left(D^{1 \times r} X\right)=p^{\prime}-1$ and $\operatorname{rank}_{D}\left(D^{1 \times s} Z\right)=\operatorname{rank}_{D}(K)-1$.

If $F \in D^{s \times r}$ (resp., $X_{2} \in D^{t \times r}$ ) is such that $Z=F X$ (resp., $\operatorname{ker}_{D}(. X)=D^{1 \times t} X_{2}$ ), then Proposition 1 shows that:

$$
L^{\prime}=\left(D^{1 \times r} X\right) /\left(D^{1 \times s} Z\right) \cong L^{\prime \prime}:=D^{1 \times r} /\left(D^{1 \times(s+t)}\left(F^{T} \quad X_{2}^{T}\right)^{T}\right) .
$$

Now, let $\tau: D^{1 \times p^{\prime}} \longrightarrow L$ (resp., $\kappa: \operatorname{ker} \varphi \longrightarrow L^{\prime}$ ) be the canonical projection onto $L$ (resp., $L^{\prime}$ ). Then, the following diagram is commutative with exact rows and columns

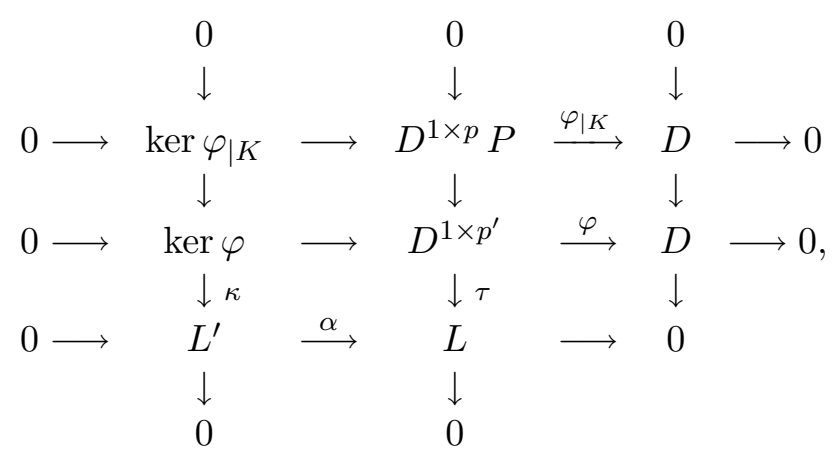

where $\alpha: L^{\prime} \longrightarrow L$ is the left $D$-isomorphism defined by:

$$
\forall \nu \in \operatorname{ker} \varphi, \quad \alpha(\kappa(\nu))=\tau(\nu) .
$$

If $\sigma: D^{1 \times r} \longrightarrow L^{\prime \prime}=D^{1 \times r} /\left(D^{1 \times(s+t)}\left(F^{T} \quad X_{2}^{T}\right)^{T}\right)$ is the canonical projection onto $L^{\prime \prime}$, then composing the left $D$-isomorphism $\beta: L^{\prime \prime} \longrightarrow L^{\prime}$ defined by $\beta(\sigma(\theta))=\kappa(\theta X)$ for all $\theta \in D^{1 \times r}$ (see Proposition 1) with $\alpha$, we get the following left $D$-isomorphism:

$$
\begin{aligned}
\gamma:=\alpha \circ \beta: L^{\prime \prime} & \longrightarrow L \\
\sigma(\theta) & \longmapsto \tau(\theta X) .
\end{aligned}
$$

Hence, using $Y P=Z=F X$, the following diagram is commutative with exact rows:

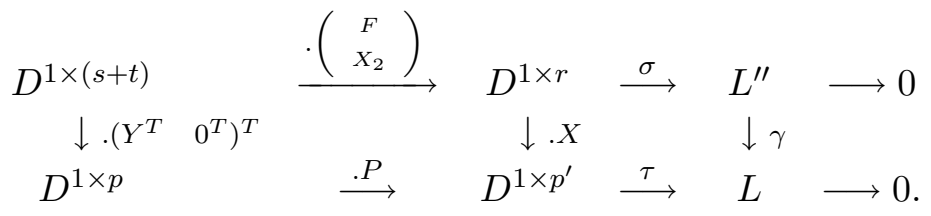

Now, using the identity $\lambda^{\star} \xi^{\star}=1$, we obtain $\left(I_{p^{\prime}}-\xi^{\star} \lambda^{\star}\right) \xi^{\star}=0$, which shows that $D^{1 \times p^{\prime}}\left(I_{p^{\prime}}-\xi^{\star} \lambda^{\star}\right) \subseteq \operatorname{ker}_{D}\left(. \xi^{\star}\right)=D^{1 \times r} X$, i.e., there exists $U \in D^{p^{\prime} \times r}$ such that:

$$
\xi^{\star} \lambda^{\star}+U X=I_{p^{\prime}}
$$


Using $\lambda^{\star}=\eta^{\star} P,(33)$ implies that $U X+\left(\xi^{\star} \eta^{\star}\right) P$ and 4 of Lemma 2 of [16] then yields:

$$
\begin{aligned}
\gamma^{-1}: L & \longrightarrow L^{\prime \prime} \\
\tau(\lambda) & \longmapsto \sigma(\lambda U) .
\end{aligned}
$$

Finally, let us point out an interesting application of the above results. We first note that the following diagram is commutative with exact rows:

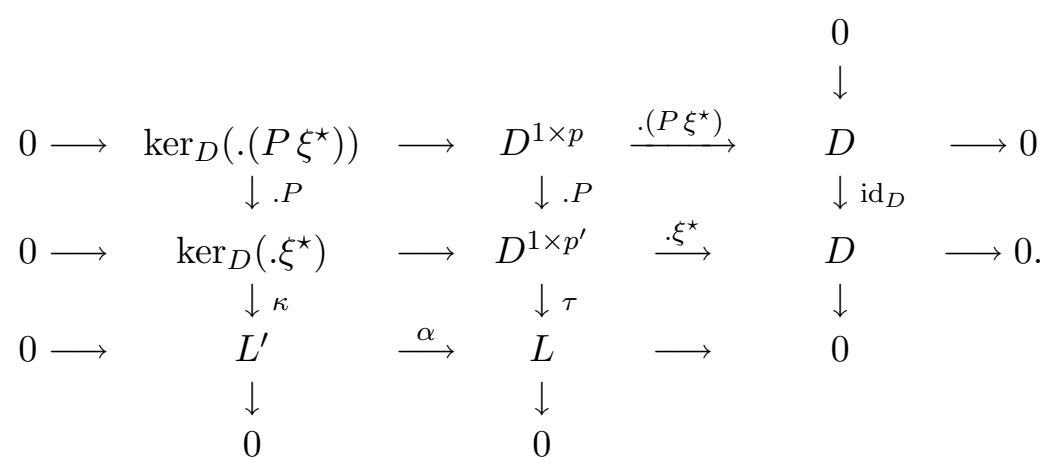

Since $\eta^{\star} P \xi^{\star}=1$, the left $D$-homomorphisms $\cdot\left(\eta^{\star} P\right): D \longrightarrow D^{1 \times p^{\prime}}$ and $\cdot \eta^{\star}: D \longrightarrow D^{1 \times p}$ satisfy $. \xi^{\star} \circ\left(. \eta^{\star} P\right)=\mathrm{id}_{D}$ and $.\left(P \xi^{\star}\right) \circ . \eta^{\star}=\mathrm{id}_{D}$, and thus the following short exact sequences

$$
0 \longrightarrow \operatorname{ker}_{D}\left(. \xi^{\star}\right) \longrightarrow D^{1 \times p^{\prime}} \stackrel{. \xi^{\star}}{\longrightarrow} D \longrightarrow 0, \quad 0 \longrightarrow \operatorname{ker}_{D}\left(.\left(P \xi^{\star}\right)\right) \longrightarrow D^{1 \times p} \stackrel{.\left(P \xi^{\star}\right)}{\longrightarrow} D \longrightarrow 0
$$

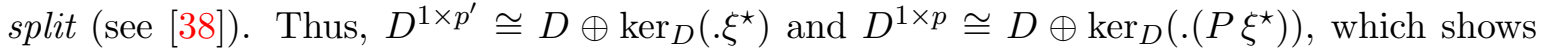
that $\operatorname{ker}_{D}\left(. \xi^{\star}\right)\left(\right.$ resp., $\left.\operatorname{ker}_{D}\left(.\left(P \xi^{\star}\right)\right)\right)$ is a stably free left $D$-module of rank $p^{\prime}-1$ (resp., $\left.p-1\right)$.

If $p^{\prime} \geq 3$, then using Theorem $6, \operatorname{ker} \varphi=\operatorname{ker}_{D}\left(\xi^{\star}\right)$ is a free left $D$-module of rank $p^{\prime}-1$. Considering a basis of $\operatorname{ker} \varphi$, there exists a full row rank matrix $X \in D^{\left(p^{\prime}-1\right) \times p^{\prime}}$ such that $\operatorname{ker} \varphi=D^{1 \times\left(p^{\prime}-1\right)} X$. Thus, $r=p^{\prime}-1, X_{2}=0, t=0$, and (32) becomes the following commutative diagram with exact rows

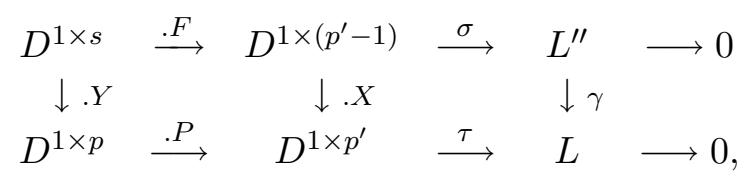

where $\gamma$ is the left $D$-isomorphism defined by (31). Hence, we have

$$
L=D^{1 \times p^{\prime}} /\left(D^{1 \times p} P\right) \cong L^{\prime \prime}=D^{1 \times\left(p^{\prime}-1\right)} /\left(D^{1 \times s} F\right),
$$

which shows that one generator of $L$ can be removed from the presentation matrix $P$.

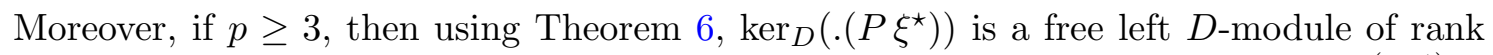
$p-1$. Considering a basis of $\operatorname{ker}_{D}\left(.\left(P \xi^{\star}\right)\right)$, there exists a full row rank matrix $Y \in D^{(p-1) \times p}$ 
such that $\operatorname{ker}_{D}\left(.\left(P \xi^{\star}\right)\right)=D^{1 \times(p-1)} Y$. Thus, $s=p-1$, and (32) becomes the following commutative diagram with exact rows

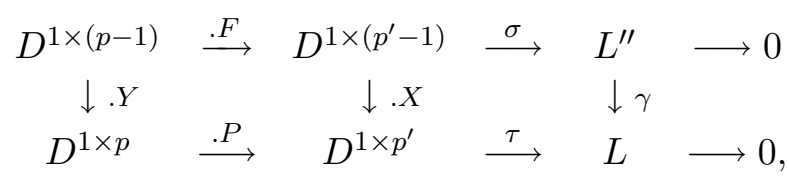

where $\gamma$ is the left $D$-isomorphism defined by (31). Hence, we have

$$
L=D^{1 \times p^{\prime}} /\left(D^{1 \times p} P\right) \cong L^{\prime \prime}=D^{1 \times\left(p^{\prime}-1\right)} /\left(D^{1 \times(p-1)} F\right),
$$

which shows that one generator and one relation of $L$ can be removed from $P$.

Remark 7. Since $\operatorname{rank}_{D}(K)=\operatorname{rank}_{D}\left(D^{1 \times p} P\right) \leq p$ and $\operatorname{rank}_{D}(K) \geq 2$ so that Theorem 8 is applicable, then we necessarily have $p \geq 2$.

We obtain the following theorem which surprisingly does not appear in [42].

Theorem 9. Let $D$ be a very simple domain, $P \in D^{p \times p^{\prime}}$, and $L=D^{1 \times p^{\prime}} /\left(D^{1 \times p} P\right)$ a finitely presented left $D$-module. If $\operatorname{rank}_{D}\left(D^{1 \times p} P\right) \geq 2$ and $p^{\prime} \geq 3$, then there exists $\bar{P} \in D^{s \times\left(p^{\prime}-1\right)}$ such that $L \cong \bar{L}:=D^{1 \times\left(p^{\prime}-1\right)} /\left(D^{1 \times s} \bar{P}\right)$. Moreover, if $p \geq 3$, then $\bar{P}$ can be chosen so that $s=p-1$, i.e., $L \cong \bar{L}:=D^{1 \times\left(p^{\prime}-1\right)} /\left(D^{1 \times(p-1)} \bar{P}\right)$.

\section{Algorithm 3 (StaffordReduction).}

- Input: A very simple domain $D$ and $P \in D^{p \times p^{\prime}}$ such that:

$$
\operatorname{rank}_{D}\left(D^{1 \times p} P\right) \geq 2 .
$$

- Output: Two matrices $\bar{P} \in D^{s \times r}$ and $X \in D^{r \times p^{\prime}}$ such that the left $D$-homomorphism

$$
\begin{aligned}
\gamma: \bar{L}:=D^{1 \times r} /\left(D^{1 \times s} \bar{P}\right) & \longrightarrow L:=D^{1 \times p^{\prime}} /\left(D^{1 \times p} P\right) \\
\sigma(\theta) & \longmapsto \tau(\theta X),
\end{aligned}
$$

is a left $D$-isomorphism, where $\sigma$ (resp., $\tau$ ) is the canonical projection onto $\bar{L}$ (resp., $L)$. If $p^{\prime} \geq 3$, then $r=p^{\prime}-1$ and, moreover, if $p \geq 3$, then $s=p-1$.

1. Pick $\eta_{1} \in D^{1 \times p}$ such that $\lambda_{1}:=\eta_{1} P \neq 0$.

2. Find $\xi_{1} \in D^{p^{\prime}}$ such that $\lambda_{1} \xi_{1} \neq 0$.

3. Compute $S \in D^{l \times p}$ such that $\operatorname{ker}_{D}\left(. \mu_{1}\right)=D^{1 \times l} S$.

4. Pick $\nu \in D^{1 \times l}$ such that $\nu(S P) \neq 0$ and define $\eta_{2}=\nu S$.

5. Find $\xi_{2} \in D^{p^{\prime}}$ such that $\left(\eta_{2} P\right) \xi_{2} \neq 0$ and define $\lambda_{2}=\eta_{2} P$. 
6. If $\lambda_{1} \xi_{2} \neq 0$, then compute $r_{1}, r_{2} \in D \backslash\{0\}$ such that $\left(\lambda_{1} \xi_{1}\right) r_{1}+\left(\lambda_{1} \xi_{2}\right) r_{2}=0$ and replace $\xi_{2}$ by $\xi_{1} r_{1}+\xi_{2} r_{2}$.

7. Compute $y_{1}, y_{2}, z_{1}, z_{2} \in D$ such that $y_{1}\left(\lambda_{1} \xi_{1}\right) z_{1}+y_{2}\left(\lambda_{2} \xi_{2}\right) z_{2}=1$.

8. Compute $\eta^{\star}=y_{1} \eta_{1}+y_{2} \eta_{2} \in D^{1 \times p}$ and $\xi^{\star}=\xi_{1} z_{1}+\xi_{2} z_{2} \in D^{p^{\prime}}$.

9. If $p^{\prime} \leq 2$, then compute $X \in D^{r \times p^{\prime}}$ such that $\operatorname{ker}_{D}\left(. \xi^{\star}\right)=D^{1 \times r} X$. Else, compute a basis of the free left $D$-module $\operatorname{ker}_{D}\left(. \xi^{\star}\right)$ of rank $p^{\prime}-1$ to get a full row rank matrix $X \in D^{\left(p^{\prime}-1\right) \times p^{\prime}}$ such that $\operatorname{ker}_{D}\left(. \xi^{\star}\right)=D^{1 \times\left(p^{\prime}-1\right)} X$, and set $r=p^{\prime}-1$.

10. If $p \leq 2$, then compute $Y \in D^{s^{\prime} \times p}$ such that $\operatorname{ker}_{D}\left(.\left(P \xi^{\star}\right)\right)=D^{1 \times s^{\prime}} Y$. Else, compute a basis of the free left $D$-module $\operatorname{ker}_{D}\left(.\left(P \xi^{\star}\right)\right)$ of rank $p-1$ to get a full row rank matrix $Y \in D^{(p-1) \times p}$ such that $\operatorname{ker}_{D}\left(.\left(P \xi^{\star}\right)\right)=D^{1 \times(p-1)} Y$, and set $s^{\prime}=p-1$.

11. Compute $Z=Y P$.

12. Left factorize $Z$ by $X$ to get $F \in D^{s^{\prime} \times r}$ such that $Z=F X$.

13. If $p \leq 2$, then find $X_{2} \in D^{t \times r}$ (possibly reduced to 0) such that $\operatorname{ker}_{D}(. X)=D^{1 \times t} X_{2}$ and define $\bar{P}=\left(\begin{array}{ll}F^{T} & X_{2}^{T}\end{array}\right)^{T}$, else $\bar{P}=F$ and set $t=0$.

14. Return $(\bar{P}, X)$ defining respectively the left $D$-module $\bar{L}=D^{1 \times\left(p^{\prime}-1\right)} /\left(D^{1 \times\left(s^{\prime}+t\right)} \bar{P}\right)$ and the left $D$-isomorphism $\gamma: \bar{L} \longrightarrow L$ given by $(31)$.

Example 8. Let $D=A_{3}(\mathbb{Q})$ and $L=D^{1 \times 3} /\left(D^{1 \times 3} P\right)$ be the left $D$-module finitely presented by the curl operator (20). We can easily check that $\operatorname{ker}_{D}(. P)=D R$, where $R=\left(\begin{array}{lll}\partial_{1} & \partial_{2} & \partial_{3}\end{array}\right)$ is the divergence operator (see Example 5). Let us apply Algorithm 2 to the left $D$-modules $M=D^{1 \times 3} /(D R) \cong K=D^{1 \times 3} P$ and $N=D^{1 \times 3}$. If we consider $\eta_{1}=\left(\begin{array}{lll}0 & 0 & 1\end{array}\right)$ and $\xi_{1}=\left(\begin{array}{lll}0 & 1 & 0\end{array}\right)^{T}$, then $d_{1}:=\eta_{1} P \xi_{1}=\partial_{1}$. Moreover, $\operatorname{ker}_{D}\left(.\left(P \xi_{1}\right)\right)=D^{1 \times 2} S$, where:

$$
S=\left(\begin{array}{ccc}
\partial_{1} & 0 & \partial_{3} \\
0 & 1 & 0
\end{array}\right)
$$

If we consider $\nu=\left(\begin{array}{ll}0 & 1\end{array}\right), \eta_{2}=\nu S=\left(\begin{array}{lll}0 & 1 & 0\end{array}\right)$, and $\xi_{2}=\left(\begin{array}{lll}0 & 0 & -1\end{array}\right)^{T}$, then $d_{2}:=\eta_{2} P \xi_{2}=$ $\partial_{1}$. Since $\eta_{1} P \xi_{2}=0$, then we need to compute a solution $\left(y_{1} y_{2} z_{1} z_{2}\right)^{T} \in D^{4}$ of $(10)$. We get $z_{1}=1, z_{2}=x_{1}+1, y_{1}=-\left(x_{1}+1\right)$, and $y_{2}=1$, which yields:

$$
\left\{\begin{array}{l}
\eta^{\star}=y_{1} \eta_{1}+y_{2} \eta_{2}=\left(\begin{array}{lll}
0 & 1 & -\left(x_{1}+1\right.
\end{array}\right), \\
\xi^{\star}=\xi_{1} z_{1}+\xi_{2} z_{2}=\left(\begin{array}{lll}
0 & 1 & -\left(x_{1}+1\right)
\end{array}\right)^{T} .
\end{array}\right.
$$

Hence, $\lambda^{\star}:=\eta^{\star} P=\left(\left(x_{1}+1\right) \partial_{2}+\partial_{3} \quad-\left(x_{1}+1\right) \partial_{1} \quad-\partial_{1}\right) \in \mathrm{U}\left(D^{1 \times 3}\right)$ and $\varphi:=\varphi_{\xi^{\star}}$ satisfies $\varphi\left(\lambda^{\star}\right)=\lambda^{\star} \xi^{\star}=1$. In other words, $\lambda^{\star}$ is a certain combination of the rows of $P$ which admits 
a right inverse over $D$. Thus, $D^{1 \times 3}=D \lambda^{\star} \oplus \operatorname{ker} \varphi$, where $\operatorname{ker} \varphi=\operatorname{ker}_{D}\left(. \xi^{\star}\right)=D^{1 \times 2} X$ and:

$$
X=\left(\begin{array}{ccc}
1 & 0 & 0 \\
0 & x_{1}+1 & 1
\end{array}\right)
$$

Since $X$ has full row rank, the rows of $X$ define a basis of the free left $D$-module $\operatorname{ker}_{D}\left(. \xi^{\star}\right)$ of rank two. Now, $\lambda^{\star}$ is a unimodular element of $K$, and thus $D^{1 \times 3} P=D \lambda^{\star} \oplus \operatorname{ker} \varphi_{\mid K}$, where

$$
\operatorname{ker} \varphi_{\mid K}=\left\{\eta P \mid \eta \in D^{1 \times 3}:(\eta P) \xi^{\star}=0\right\}=\operatorname{ker}_{D}\left(.\left(P \xi^{\star}\right)\right) P,
$$

and $\operatorname{ker}_{D}\left(.\left(P \xi^{\star}\right)\right)=D^{1 \times 2} Y$, where the matrix $Y \in D^{2 \times 3}$ is defined by:

$$
Y=\left(\begin{array}{ccc}
-1 & -\left(x_{1}+1\right) \partial_{2}-\partial_{3} & \left(x_{1}+1\right)\left(\left(x_{1}+1\right) \partial_{2}+\partial_{3}\right) \\
0 & -\partial_{1} & \left(x_{1}+1\right) \partial_{1}+2
\end{array}\right)
$$

Since $Y$ has full row rank, the rows of $Y$ define a basis of the free left $D$-module $\operatorname{ker}_{D}\left(.\left(P \xi^{\star}\right)\right)$ of rank two. Then, $\operatorname{ker} \varphi_{\mid K}=D^{1 \times 2} Z$, where $Z=Y P$. Now, let $F \in D^{2 \times 2}$ be the matrix such that $Z=F X$, i.e.:

$$
F=\left(\begin{array}{cc}
-\left(\partial_{3}+\left(x_{1}+1\right) \partial_{2}\right)^{2} & \left(\left(x_{1}+1\right) \partial_{2}+\partial_{3}\right) \partial_{1}-\partial_{2} \\
-\left(\left(x_{1}+1\right) \partial_{2}+\partial_{3}\right) \partial_{1}-2 \partial_{2} & \partial_{1}^{2}
\end{array}\right)
$$

Since $\operatorname{ker}_{D}(. X)=0$, Proposition 1 shows that:

$$
L=D^{1 \times 3} /\left(D^{1 \times 3} P\right) \cong L^{\prime}:=\left(D^{1 \times 2} X\right) /\left(D^{1 \times 2} Z\right) \cong \bar{L}:=D^{1 \times 2} /\left(D^{1 \times 2} F\right) .
$$

The left $D$-isomorphism $\gamma: \bar{L} \longrightarrow L$ is then defined by $\gamma(\sigma(\theta))=\tau(\theta X)$ for all $\theta \in D^{1 \times 2}$, where $\sigma: D^{1 \times 2} \longrightarrow \bar{L}$ (resp., $\tau: D^{1 \times 3} \longrightarrow L$ ) is the canonical projection onto $\bar{L}$ (resp., $L$ ). Moreover, $\gamma^{-1}: L \longrightarrow \bar{L}$ is defined by $\gamma^{-1}(\tau(\lambda))=\sigma(\lambda U)$ for all $\lambda \in D^{1 \times 3}$, where:

$$
U=\left(\begin{array}{cc}
1 & 0 \\
-\left(x_{1}+1\right) \partial_{2}-\partial_{3} & \partial_{1} \\
\left(x_{1}+1\right)\left(\left(x_{1}+1\right) \partial_{2}+\partial_{3}\right) & -\left(x_{1}+1\right) \partial_{1}+1
\end{array}\right)
$$

Finally, since $\operatorname{ker}_{D}(. F)=D\left(-\partial_{1} \partial_{3}+\left(x_{1}+1\right) \partial_{2}\right)$, we have $\operatorname{rank}_{D}\left(D^{1 \times 2} F\right)=1$, and Algorithm 2 cannot be applied again to $\bar{L}$.

Finally, let us compute the inverse of the left $D$-isomorphism $\alpha: L^{\prime} \longrightarrow L$ defined by $\alpha(\kappa(\nu))=\tau(\nu)$ for all $\nu \in D^{1 \times r} X$ (see (30)). This result will be used in the next section.

Now, using the identity $\eta^{\star} P \xi^{\star}=1$, we obtain $\left(I_{p}-P \xi^{\star} \eta^{\star}\right) P \xi^{\star}=0$, which shows that $D^{1 \times p}\left(I_{p}-P \xi^{\star} \eta^{\star}\right) \subseteq \operatorname{ker}_{D}\left(.\left(P \xi^{\star}\right)\right)=D^{1 \times s} Y$, i.e., there exists $V \in D^{p \times s}$ such that:

$$
P \xi^{\star} \eta^{\star}+V Y=I_{p}
$$

$\mathrm{RR} \mathrm{n}^{\circ} 8225$ 
Multiplying $P$ on the right of (37) and on the left of (33) and subtracting the result, we get:

$$
P U X=V Y P \text {. }
$$

Now, we claim that $\alpha^{-1}$ is the left $D$-homomorphism defined by:

$$
\begin{aligned}
\omega: L & \longrightarrow L^{\prime} \\
\tau(\lambda) & \longmapsto \kappa(\lambda U X) .
\end{aligned}
$$

Using (38) and $Z=Y P$, we first check that $\omega$ is well-defined:

$$
\forall \eta \in D^{1 \times p}, \quad \omega(\tau(\lambda+\eta P))=\kappa(\lambda U X)+\kappa((\eta V) Z)=\kappa(\lambda U X)=\omega(\tau(\lambda)) .
$$

Finally, using (33), $\lambda^{\star}=\eta^{\star} P$, and $X \xi^{\star}=0$, we can check that

$$
\begin{aligned}
(\alpha \circ \omega)(\tau(\lambda)) & =\alpha(\kappa(\lambda U X))=\tau(\lambda U X)=\tau(\lambda)-\tau\left(\left(\lambda \xi^{\star} \eta^{\star}\right) P\right)=\tau(\lambda), \\
(\omega \circ \alpha)(\kappa(\theta X)) & =\omega(\tau(\theta X))=\kappa(\theta X U X)=\kappa\left(\theta X\left(I_{p^{\prime}}-\xi^{\star} \eta^{\star} P\right)\right)=\kappa(\theta X),
\end{aligned}
$$

which shows that $\omega=\alpha^{-1}$.

\section{$5 \quad$ Efficient generation of finitely generated modules}

We have the following interesting corollary of Theorem 9 .

Corollary 2. Let $L=D^{1 \times p^{\prime}} /\left(D^{1 \times p} P\right)$ be a torsion left $D$-module.

1. If $p^{\prime} \geq 3$, then there exists $\bar{P} \in D^{l \times 2}$ such that $L \cong \bar{L}:=D^{1 \times 2} /\left(D^{1 \times l} \bar{P}\right)$. In particular, $L$ can be generated by two elements. Moreover, $l$ can be chosen such that:

$$
l=p-p^{\prime}+2 .
$$

2. There exist a projective left ideal $I$ of $D$ and a left submodule $J$ of $I$ such that $L \cong I / J$. In other words, $L$ is a homomorphic image of a projective left ideal I of $D$ (which can be generated by two elements).

Proof. 1. Since $L$ is a torsion left $D$-module, by $1 \Rightarrow 3$ of Lemma $1, \operatorname{rank}_{D}(L)=0$, which yields $\operatorname{rank}_{D}\left(D^{1 \times p} P\right)=p^{\prime} \geq 3$. Applying $p^{\prime}-2$ times Theorem 9 , we obtain $\bar{P} \in D^{l \times 2}$ such that $L \cong \bar{L}:=D^{1 \times 2} /\left(D^{1 \times l} \bar{P}\right)$, which proves the first part of 1 . Finally, using that

$$
p \geq \operatorname{rank}_{D}\left(D^{1 \times p} P\right)=p^{\prime} \geq 3
$$

then Theorem 9 shows that $l$ can be chosen such that $l=p-p^{\prime}+2$.

2. If $p^{\prime}=1$, then $L=D /\left(D^{1 \times p} P\right)$, which proves 2 with $I=D$ and $J=D^{1 \times p} P$. Now, if $p^{\prime}>1$, using 1 , we may assume that $p^{\prime}=2$, i.e., $L=D^{1 \times 2} /\left(D^{1 \times p} P\right)$. Let $R \in D^{q \times p}$ 
(possibly $R=0$ and $q=0$ ) be such that $\operatorname{ker}_{D}(. P)=D^{1 \times q} R, M=D^{1 \times p} /\left(D^{1 \times q} R\right)$, $\pi$ the canonical projection onto $M\left(\pi=\mathrm{id}_{M}\right.$ if $\left.R=0\right)$, and $\iota: M \longrightarrow D^{1 \times 2}$ the injection defined by $\iota(\pi(\lambda))=\lambda P$ for all $\lambda \in D^{1 \times p}$. The following diagram is commutative with exact rows:

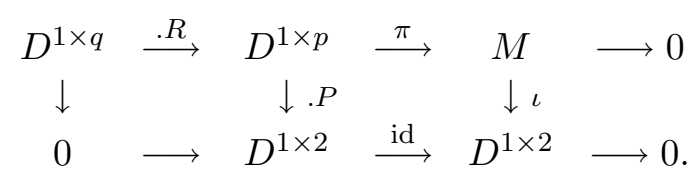

Since $\operatorname{rank}_{D}\left(D^{1 \times p} P\right)=2$, the proof of Theorem 8 for $K=\iota(M)$ shows that there exist $\eta^{\star} \in D^{1 \times p}$ and $\xi^{\star} \in D^{2}$ such that $\varphi:=\varphi_{\xi^{\star}}$ satisfies:

$$
\varphi\left(\iota\left(\pi\left(\eta^{\star}\right)\right)\right)=\eta^{\star} P \xi^{\star}=1 .
$$

Thus, if $\lambda^{\star}:=\eta^{\star} P$, then $D^{1 \times p} P=D \lambda^{\star} \oplus N^{\prime} \subseteq D^{1 \times 2}=D \lambda^{\star} \oplus N$, where $N:=\operatorname{ker} \varphi=$ $\operatorname{ker}_{D}\left(. \xi^{\star}\right)$ and $N^{\prime}:=\operatorname{ker} \varphi_{\mid K}=\operatorname{ker}_{D}\left(.\left(P \xi^{\star}\right)\right) P$. This implies:

$$
L=\left(D \lambda^{\star} \oplus N\right) /\left(D \lambda^{\star} \oplus N^{\prime}\right) \cong L^{\prime}:=N / N^{\prime} .
$$

Let $\kappa: N \longrightarrow L^{\prime}=N / N^{\prime}$ (resp., $\tau: D^{1 \times 2} \longrightarrow L$ ) be the canonical projection onto $L^{\prime}$ (resp., $L)$. Then, the above left $D$-isomorphism is defined by (30), namely:

$$
\begin{aligned}
\alpha: L^{\prime} & \longrightarrow L \\
\kappa(\nu) & \longmapsto \tau(\nu) .
\end{aligned}
$$

Since $D^{1 \times 2}=D \lambda^{\star} \oplus N \cong D \oplus N, N$ is a projective left $D$-module of rank one, and thus $N$ is isomorphic to a projective left ideal of $D$, which can be generated by two elements (see Section 3.2). Note that if $N$ is a free left $D$-module of rank one, then $N$ is isomorphic to a principal left ideal of $D$ (i.e., generated by one element). Let $X \in D^{r \times 2}$ (resp., $Y \in D^{s \times p}$ ) be such that $N=\operatorname{ker}_{D}\left(\xi^{\star}\right)=D^{1 \times r} X$ (resp., $\left.\operatorname{ker}_{D}\left(.\left(P \xi^{\star}\right)\right)=D^{1 \times s} Y\right)$. If $Z=Y P \in D^{s \times 2}$, then $N^{\prime}=\operatorname{ker}_{D}\left(.\left(P \xi^{\star}\right)\right) P=D^{1 \times s} Z$, and thus $N / N^{\prime}=\left(D^{1 \times r} X\right) /\left(D^{1 \times s} Z\right)$. Since $N^{\prime} \subseteq N$, there exists $F \in D^{s \times r}$ such that $Z=F X$ and the injection $i: N^{\prime} \longrightarrow N$ is defined by:

$$
\forall \theta \in D^{1 \times s}, \quad i(\theta Z)=(\theta F) X .
$$

Let $X_{2} \in D^{t \times r}$ (resp., $Z_{2} \in D^{u \times s}$ ) be such that $\operatorname{ker}_{D}(. X)=D^{1 \times t} X_{2}$ (resp., $\operatorname{ker}_{D}(. Z)=$ $D^{1 \times u} Z_{2}$ ). Then, the following diagram is commutative with exact rows

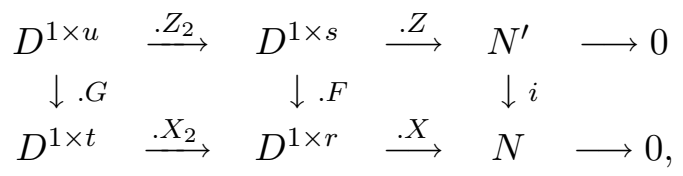

where $G \in D^{u \times t}$ satisfies $Z_{2} F=G X_{2}$. Since $N$ and $N^{\prime}$ are two left $D$-submodules of $D^{1 \times 2}$, they are torsion-free. Hence, $N_{2}:=D^{1 \times r} /\left(D^{1 \times t} X_{2}\right) \cong D^{1 \times r} X=N$ and $N_{2}^{\prime}:=$ 
$D^{1 \times s} /\left(D^{1 \times u} Z_{2}\right) \cong D^{1 \times s} Z=N^{\prime}$ are also torsion-free left $D$-modules of rank one. Considering a minimal parametrization of $N_{2}$ and of $N_{2}^{\prime}$ (see [11]), there exist two matrices $B \in D^{r}$ and $C \in D^{s}$ such that $N_{2} \cong I:=D^{1 \times r} B$ and $N_{2}^{\prime} \cong H:=D^{1 \times s} C$, where $I$ and $H$ are two finitely generated left ideals of $D$. To get such minimal parametrizations, we need to pick $B \in D^{r}$ (resp., $\left.C \in D^{s}\right)$ such that $B D \subseteq \operatorname{ker}_{D}\left(X_{2}\right.$.) (resp., $C D \subseteq \operatorname{ker}_{D}\left(Z_{2}\right.$.)). Then, by definition of the minimal parametrizations, the next diagram is commutative with exact rows

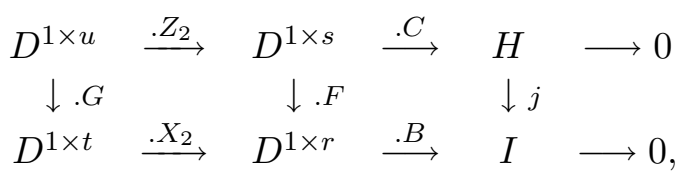

where $j \in \operatorname{hom}_{D}(H, I)$ is defined by:

$$
\forall \theta \in D^{1 \times s}, \quad j(\theta C)=(\theta F) B .
$$

Let us note that we then have the following left $D$-isomorphisms

$$
\begin{aligned}
\varepsilon: H & \longrightarrow N^{\prime} \quad \varepsilon^{\prime}: I \quad \longrightarrow N \\
\theta C & \longmapsto \theta Z, \quad v B
\end{aligned}
$$

which satisfy $\varepsilon^{\prime} \circ j=i \circ \varepsilon$, where $i$ and $j$ are respectively defined by (40) and (41).

If we consider the left subideal $J=\operatorname{im} j=D^{1 \times s}(F B)$ of $I$, then we have:

$$
\text { coker } j=I / J=\left(D^{1 \times r} B\right) /\left(D^{1 \times s}(F B)\right) .
$$

Let $\delta: I \longrightarrow I / J$ be the canonical projection onto $I / J$. Then, the following diagram is commutative with exact rows and columns

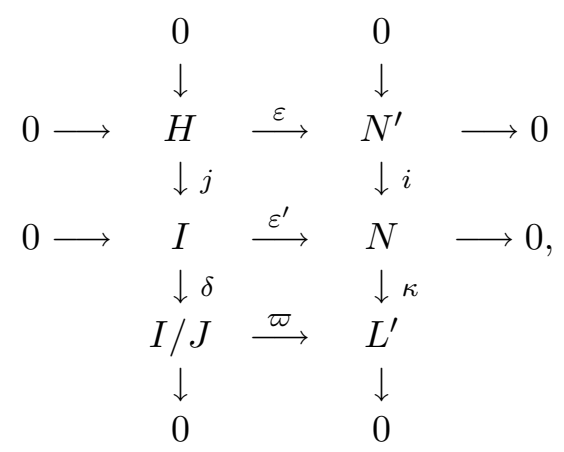

where $\varpi \in \operatorname{hom}_{D}\left(I / J, L^{\prime}\right)$ is the left $D$-isomorphism defined by:

$$
\begin{aligned}
& \varpi: I / J \quad \longrightarrow L^{\prime} \\
& \delta(v B)) \quad \longmapsto\left(\varepsilon^{\prime}(v B)\right)=\kappa(v X) .
\end{aligned}
$$

$\mathrm{RR} \mathrm{n}^{\circ} 8225$ 
Composing $\varpi$ with the left $D$-isomorphism $\alpha \in \operatorname{hom}_{D}\left(L^{\prime}, L\right)$ defined by (30), we then obtain the following left $D$-isomorphism:

$$
\begin{aligned}
\chi=\alpha \circ \varpi: I / J & \longrightarrow L \\
\delta(v B) & \longmapsto \tau(v X) .
\end{aligned}
$$

Let us now explicitly characterize the inverse $\chi^{-1}=\varpi^{-1} \circ \alpha^{-1}$ of $\chi$. We first note that $\varpi^{-1}: L^{\prime} \longrightarrow I / J$ is defined by $\varpi^{-1}(\kappa(v X))=\delta(v B)$ for all $v \in D^{1 \times r}$. Secondly, $\alpha^{-1}$ is defined by (39), i.e., $\alpha^{-1}(\tau(\lambda))=\kappa(\lambda U X)$, where $U \in D^{2 \times r}$ is defined by (33), i.e., by $I_{2}-\xi^{\star} \lambda^{\star}=U X$. Thus, the left $D$-isomorphism $\chi^{-1}$ is finally defined by:

$$
\forall \lambda \in D^{1 \times 2}, \quad \chi^{-1}(\tau(\lambda))=\varpi^{-1}\left(\alpha^{-1}(\tau(\lambda))\right)=\varpi^{-1}(\kappa(\lambda U X))=\delta((\lambda U B) .
$$

Remark 8. We note that 1 of Corollary 2 does not give in general the most accurate bound for the number of generators of a torsion left $D$-module because cyclic torsion left $D$-modules exist (namely, torsion left $D$-modules of the form $D / J$, where $J$ is a left ideal of $D$ ).

Example 9. Many linear PD systems coming from mathematical physics or engineering science are defined by means of a square full row rank matrix $P \in D^{p^{\prime} \times p^{\prime}}$ of $\mathrm{PD}$ operators. Applying 1 of Corollary 2 to $P \in D^{p^{\prime} \times p^{\prime}}$, we obtain that these linear PD systems are equivalent to linear PD systems defined by $\bar{P} \in D^{2 \times 2}$, i.e., to linear PD systems defined by (at most) two unknowns and two PD equations. For an algorithm computing $\bar{P} \in D^{2 \times 2}$, see $[7,15]$.

Example 10. Let $D=A\langle\partial\rangle$ be a ring of OD operators, where $A=k[t]$ or $A=k \llbracket t \rrbracket$ and $k$ is a field of characteristic zero, or $A=k\{t\}$, where $k \in\{\mathbb{R}, \mathbb{C}\}, E \in A^{n \times n}, R=\partial I_{n}-E \in D^{n \times n}$, and $M=D^{1 \times n} /\left(D^{1 \times n} R\right)$. If $\left\{f_{j}\right\}_{j=1, \ldots, n}$ is the standard basis of $D^{1 \times n}, \pi: D^{1 \times n} \longrightarrow M$ the canonical projection onto $M, x_{j}=\pi\left(f_{j}\right)$ for $j=1, \ldots, n$, and $x=\left(x_{1} \ldots x_{n}\right)^{T}$, then $R x=0$ (see Section 2). By 1 of Corollary 2, the torsion left $D$-module $M$ can be generated by two elements $y_{1}=\pi\left(G_{1}\right)=G_{1} x$ and $y_{2}=\pi\left(G_{2}\right)=G_{2} x$, where $G_{i} \in D^{1 \times n}$ for $i=1,2$. Equivalently, the matrix $\left(\begin{array}{lll}G_{1}^{T} & G_{2}^{T} & R^{T}\end{array}\right)^{T}$ admits a left inverse (see, e.g., [7, 15]), i.e., there exist $S_{1} \in D^{n}, S_{2} \in D^{n}$, and $S_{3} \in D^{n \times n}$ such that:

$$
S_{1} G_{1}+S_{2} G_{2}+S_{3} R=I_{n}
$$

Now, if we consider the linear OD control system $\dot{x}=E x+F u$ (see, e.g., [22]), where $F \in A^{n \times m}$, the above remark shows that that there exists $G=\left(\begin{array}{ll}G_{1}^{T} & G_{2}^{T}\end{array}\right)^{T} \in A^{2 \times n}$ such that (42) holds. Thus, the following linear OD control system

$$
\left\{\begin{array}{l}
\dot{x}=E x+F u, \\
y=G x
\end{array}\right.
$$

$\mathrm{RR} \mathrm{n}^{\circ} 8225$ 
is observable (see, e.g., [22]), namely, the state $x$ of (43) can be expressed in terms of $y_{1}, y_{2}, u$, and their derivatives. Indeed, combining $R x=F u$ and (42), we get $x=S_{1} y_{1}+S_{2} y_{2}+S_{3} F u$. Hence, 1 of Corollary 2 shows that there exist two outputs $y_{1}=G_{1} x$ and $y_{2}=G_{2} x$ of the linear OD control system $\dot{x}=E x+F u$ such that its state $x$ is observable by means of $y_{1}$, $y_{2}$, and $u$.

\section{Algorithm 4 (ProjectiveIdealPresentation).}

- Input: A very simple domain $D$ and $P \in D^{p \times p^{\prime}}$ such that the finitely presented left $D$-module $L=D^{1 \times p^{\prime}} /\left(D^{1 \times p} P\right)$ is torsion.

- Output: Three matrices $B \in D^{x}, B^{\prime} \in D^{y}$, and $W \in D^{x \times p^{\prime}}$ such that

$$
\begin{aligned}
\chi: K:=\left(D^{1 \times x} B\right) /\left(D^{1 \times y} B^{\prime}\right) & \longrightarrow L \\
\delta(v B) & \longmapsto \tau(v W)
\end{aligned}
$$

is an isomorphism of left $D$-modules, where $\delta: D^{1 \times x} B \longrightarrow K\left(\right.$ resp., $\tau: D^{1 \times p^{\prime}} \longrightarrow L$ ) is the canonical projection onto $K$ (resp., $L$ ).

1. If $p^{\prime}=1$, then return $B^{\prime}=P, B=1, W=1$ (i.e., $x=1, y=p$ ).

2. Apply Algorithm 3 to $P$ to obtain two matrices $\bar{P} \in D^{s \times 2}$ and $X \in D^{2 \times p^{\prime}}$ such that the left $D$-homomorphism $\gamma: \bar{L}=D^{1 \times 2} /\left(D^{1 \times s} \bar{P}\right) \longrightarrow L$ defined by (34) is an isomorphism.

3. Apply Algorithm 2 to the matrices $R:=\bar{R} \in D^{t \times s}$ satisfying $\operatorname{ker}_{D}(. \bar{P})=D^{1 \times t} \bar{R}$, $R^{\prime}=0$, and $P:=\bar{P}$ to obtain $\eta^{\star} \in D^{1 \times s}$ and $\xi^{\star} \in D^{2}$ such that $\eta^{\star} \bar{P} \xi^{\star}=1$.

4. Compute $\bar{X} \in D^{x \times 2}$ such that $\operatorname{ker}_{D}\left(. \xi^{\star}\right)=D^{1 \times x} \bar{X}$.

5. Compute $\bar{Y} \in D^{y \times s}$ such that $\operatorname{ker}_{D}\left(.\left(\bar{P} \xi^{\star}\right)\right)=D^{1 \times y} \bar{Y}$.

6. Compute $\bar{Z}=\bar{Y} \bar{P} \in D^{y \times 2}$.

7. Left factorize $\bar{Z}$ by $\bar{X}$ to get $\bar{F} \in D^{y \times x}$ such that $\bar{Z}=\bar{F} \bar{X}$.

8. Compute $\bar{X}_{2} \in D^{z \times x}$ such that $\operatorname{ker}_{D}(. \bar{X})=D^{1 \times z} \bar{X}_{2}$.

9. Compute $B \in D^{x}$ such that $B D \subseteq \operatorname{ker}_{D}\left(\bar{X}_{2}.\right)$.

10. Compute $B^{\prime}=\bar{F} B \in D^{y}$.

11. Compute $W=\bar{X} X \in D^{x \times p^{\prime}}$.

12. Return the matrices $B, B^{\prime}$, and $W$. 
Example 11. Let $D=A_{1}(\mathbb{Q})$ and $L=D^{1 \times 2} /\left(D^{1 \times 2} P\right)$ be the left $D$-module finitely presented by the matrix $P$ defined by:

$$
P=\left(\begin{array}{cc}
t^{2} & t \\
t \partial+2 & \partial
\end{array}\right)
$$

Since $P$ has full row rank, $L$ is a torsion left $D$-module. Corollary 2 then shows that $L \cong I / J$, where $I$ and $J$ are two left ideals of $D$ such that $J \subseteq I$, both of which can be generated by two elements. Let us compute $I$ and $J$. If we consider $\eta^{\star}=\left(\begin{array}{ll}\partial & -t\end{array}\right)$ and $\xi^{\star}=\left(\begin{array}{ll}0 & 1\end{array}\right)^{T}$, then $\eta^{\star} P \xi^{\star}=1$. Now, $\operatorname{ker}_{D}\left(. \xi^{\star}\right)=D X, \operatorname{ker}_{D}\left(.\left(P \xi^{\star}\right)\right)=D^{1 \times 2} Y, Z=Y P, Z=F X$, where:

$$
X=\left(\begin{array}{ll}
1 & 0
\end{array}\right), \quad Y=\left(\begin{array}{cc}
t \partial-1 & -t^{2} \\
\partial^{2} & -t \partial-2
\end{array}\right), \quad Z=\left(\begin{array}{cc}
-t^{2} & 0 \\
-t \partial-2 & 0
\end{array}\right), \quad F=\left(\begin{array}{c}
-t^{2} \\
-t \partial-2
\end{array}\right) .
$$

Since $\operatorname{ker}_{D}(. X)=0$, i.e., $X_{2}=0$, then $\operatorname{ker}_{D}\left(X_{2}.\right)=D$, which shows that $B=1$ is a minimal parametrization of $N=D X \cong D$ and $I=D B=D$. Thus, $B^{\prime}=F B=-\left(t^{2} t \partial+2\right)^{T}$, which shows that $L \cong K:=D / J$, where $J=D t^{2}+D(t \partial+2)$. We note that $J$ is the cyclic left $D$-module which is the annihilator of the derivative of the Dirac distribution (see Example 1.2.9 of [33]). The left $D$-isomorphism $\chi: K \longrightarrow L$ is defined by $\chi(\delta(v))=\kappa(v X)$ for all $v \in D$, where $\delta: D \longrightarrow K$ (resp., $\tau: D^{1 \times 2} \longrightarrow L$ ) is the canonical projection onto $K$ (resp., $L)$. Thus, $L$ is generated by $\tau(X)=\chi(\delta(1))$ and we can easily check that $\tau\left(\left(\begin{array}{ll}0 & 1\end{array}\right)\right)=0$ (for more details, see [36]). Finally, the matrix $U=\left(\begin{array}{ll}1 & 0\end{array}\right)^{T}$ satisfies $I_{2}-\left(\xi^{\star} \eta^{\star}\right) P=U X$, which shows that $\chi^{-1}: L \longrightarrow K$ is defined by $\chi^{-1}(\tau(\lambda))=\delta(\lambda U)$ for all $\lambda \in D^{1 \times 2}$.

Example 12. Corollary 2 can be understood as a generalization of the standard cyclic vector theorem for linear PD systems (see [10] and the references therein). 1 of Corollary 2 shows that every finitely generated torsion left $D$-module $L$ can be generated by two elements. Moreover, 2 of Corollary 2 states that $L \cong I / J$, where $I$ is a projective ideal of $D$ and $J$ is a left $D$-submodule of $I$. Now, if $D=A\langle\partial\rangle$ is a ring of OD operators with coefficients in a differential field $A$, then $D$ is a principal ideal domain (see, e.g., [30]), and thus $I$ and $J$ are two principal ideals of $D$, i.e., there exist $d_{1}, d_{2} \in D$ such that $L \cong\left(D d_{1}\right) /\left(D d_{2}\right) \cong D /\left(D d_{3}\right)$, where $d_{3} \in D$ is such that $d_{1}=d_{3} d_{2}$. The element $d_{3}$, which is not uniquely determined by $L$, can be computed by means of a Jacobson normal form of the presentation matrix $P$ of $L$.

Let us illustrate with a simple example that Algorithm 4 can be used to compute $d_{3}$. Let $D=B_{1}(\mathbb{Q}), a_{i j} \in A=\mathbb{Q}(t)$ for $i, j=1,2, E=\left(a_{i j}\right)_{i, j=1,2}$, and $L=D^{1 \times 2} /\left(D^{1 \times 2} P\right)$, where:

$$
P=\partial I_{2}-E=\left(\begin{array}{cc}
\partial-a_{11} & -a_{12} \\
-a_{21} & \partial-a_{22}
\end{array}\right)
$$

The left $D$-module $L$ corresponds to the linear OD system $\dot{x}=E x$. Let us suppose that $a_{12} \neq 0$ (a similar result holds if $a_{21} \neq 0$ ), which implies $a_{12}^{-1} \in A$. Choosing $\eta^{\star}=\left(\begin{array}{ll}1 & 0\end{array}\right)$, 
$\xi^{\star}=\left(\begin{array}{ll}0 & -a_{12}^{-1}\end{array}\right)^{T}$, we get $\eta^{\star} P \xi^{\star}=1$. Then, $\operatorname{ker}_{D}\left(. \xi^{\star}\right)=D X$, where $X=\left(\begin{array}{ll}1 & 0\end{array}\right)$, and $\operatorname{ker}_{D}\left(.\left(P \xi^{\star}\right)\right)=D Y$, where $Y=\left(\left(\partial-a_{22}\right) a_{12}^{-1} \quad 1\right)$, since $P \xi^{\star}=\left(\begin{array}{ll}1 & -\left(\partial-a_{22}\right) a_{12}^{-1}\end{array}\right)^{T}$. Then, $Z=Y P=\left(\left(\partial-a_{22}\right) a_{12}^{-1}\left(\partial-a_{11}\right)-a_{21} \quad 0\right)=F X$, where $F=\left(\partial-a_{22}\right) a_{12}^{-1}\left(\partial-a_{11}\right)-a_{21}$, which shows that:

$$
L \cong\left(D^{1 \times 2} X\right) /\left(D^{1 \times 2} Z\right) \cong D /(D F) .
$$

In case $a_{11} \neq a_{22}$ and $a_{12}=a_{21}=0$, then considering $\eta_{1}=\left(\begin{array}{ll}1 & 0\end{array}\right), \xi_{1}=\left(\begin{array}{ll}1 & 0\end{array}\right)^{T}$, $d_{1}:=\eta_{1} P \xi_{1}=\partial-a_{11}, \operatorname{ker}_{D}\left(.\left(P \xi_{1}\right)\right)=D\left(\begin{array}{ll}0 & 1\end{array}\right), \eta_{2}=\left(\begin{array}{ll}0 & 1\end{array}\right), \xi_{2}=\left(\begin{array}{ll}0 & 1\end{array}\right)^{T}, d_{2}:=\eta_{2} P \xi_{2}=$ $\partial-a_{22}, \eta_{2} P \xi_{1}=0, y_{1} d_{1} z_{1}+y_{2} d_{2} z_{2}=1$, where $-y_{1}=y_{2}=\left(a_{11}-a_{22}\right)^{-1}, z_{1}=z_{2}=1$, and thus $\eta^{\star}=y_{1} \eta_{1}+y_{2} \eta_{2}=\left(a_{11}-a_{22}\right)^{-1}\left(\begin{array}{ll}-1 & 1\end{array}\right)$ and $\xi=\xi_{1} z_{1}+\xi_{2} z_{2}=\left(\begin{array}{ll}1 & 1\end{array}\right)^{T}$ are such that $\eta^{\star} P \xi=1$. Then, Algorithm 4 gives $\operatorname{ker}_{D}(. \xi)=D X$, where $X=\left(\begin{array}{ll}1 & -1\end{array}\right)$, $\operatorname{ker}_{D}(.(P \xi))=D Y$, where $Y=\left(\partial-a_{22}-\partial+a_{11}\right)\left(a_{11}-a_{22}\right)^{-1}, Z=Y P=F X$, where $F=\left(\partial-a_{22}\right)\left(a_{11}-a_{22}\right)^{-1}\left(\partial-a_{11}\right)=\left(\partial-a_{11}\right)\left(a_{11}-a_{22}\right)^{-1}\left(\partial-a_{22}\right)$, and thus we obtain:

$$
L \cong(D X) /(D Z) \cong D /(D F) \text {. }
$$

Now, if $a_{11}=a_{22}$ and $a_{12}=a_{21}=0$, then considering $\eta_{1}=\left(\begin{array}{ll}1 & 0\end{array}\right), \xi_{1}=\left(\begin{array}{ll}1 & 0\end{array}\right)^{T}$, $d_{1}:=\eta_{1} P \xi_{1}=\partial-a_{11}, \operatorname{ker}_{D}\left(.\left(P \xi_{1}\right)\right)=D\left(\begin{array}{ll}0 & 1\end{array}\right), \eta_{2}=\left(\begin{array}{ll}0 & 1\end{array}\right), \xi_{2}=\left(\begin{array}{ll}0 & 1\end{array}\right)^{T}, d_{2}:=\eta_{2} P \xi_{2}=$ $\partial-a_{11}, \eta_{2} P \xi_{1}=0, y_{1} d_{1} z_{1}+y_{2} d_{2} z_{2}=1$, where $y_{1}=z_{2}=1, z_{1}=t, y_{2}=-t$, and thus $\eta^{\star}=y_{1} \eta_{1}+y_{2} \eta_{2}=\left(\begin{array}{ll}1 & -t\end{array}\right)$ and $\xi=\xi_{1} z_{1}+\xi_{2} z_{2}=\left(\begin{array}{ll}t & 1\end{array}\right)^{T}$ are such that $\eta^{\star} P \xi=1$. Then, Algorithm 4 gives $\operatorname{ker}_{D}(. \xi)=D X$, where $X=\left(\begin{array}{ll}-1 & t)\end{array}\right) \operatorname{ker}_{D}(.(P \xi))=D Y$, where $Y=\left(-\partial-a_{11} \quad t \partial-a_{11} t+2\right), Z=Y P=F X$, where $F=\left(\partial-a_{11}\right)^{2}$, and thus we obtain:

$$
L \cong(D X) /(D Z) \cong D /(D F) .
$$

Corollary 3. Let $P \in D^{p \times p^{\prime}}$ be a full row rank matrix and $L=D^{1 \times p^{\prime}} /\left(D^{1 \times p} P\right)$. Then, there exists $\bar{P} \in D^{2 \times\left(p^{\prime}-p+2\right)}$ such that:

$$
L \cong \bar{L}:=D^{1 \times\left(p^{\prime}-p+2\right)} /\left(D^{1 \times 2} \bar{P}\right) .
$$

If $p^{\prime}-p \geq 1$ and the right $D$-module $\operatorname{ext}_{D}^{1}(L, D):=D^{p} /\left(P D^{p^{\prime}}\right)$ is cyclic, then there exists $R^{\prime} \in D^{1 \times\left(p^{\prime}-p+1\right)}$ such that $L \cong M^{\prime}:=D^{1 \times\left(p^{\prime}-p+1\right)} /\left(D R^{\prime}\right)$.

Proof. Applying the contravariant left exact functor $\operatorname{hom}_{D}(\cdot, D)$ to the short exact sequence

$$
0 \longrightarrow D^{1 \times p} \stackrel{. P}{\longrightarrow} D^{1 \times p^{\prime}} \stackrel{\sigma}{\longrightarrow} L \longrightarrow 0
$$

we get the following short exact sequence of right $D$-modules (see, e.g., [11, 38]):

$$
0 \longleftarrow \operatorname{ext}_{D}^{1}(L, D) \stackrel{\varpi}{\longleftarrow} D^{p} \stackrel{P .}{\longleftarrow} D^{p^{\prime}} \longleftarrow \operatorname{hom}_{D}(L, D) \longleftarrow 0 .
$$

Now, by a version of (2) for right $D$-modules, we have $\operatorname{hom}_{D}\left(\operatorname{ext}_{D}^{1}(L, D), D\right) \cong \operatorname{ker}_{D}(. P)=0$, which shows that $\operatorname{ext}_{D}^{1}(L, D)$ is a torsion right $D$-module by $2 \Rightarrow 1$ of Lemma 1 . Thus, it can be generated by two elements by 1 of Corollary 2 . Let $\Lambda \in D^{p \times 2}$ be such that 
$\left\{\varpi\left(\Lambda_{\bullet}\right)\right\}_{i=1,2}$ generates $\operatorname{ext}_{D}^{1}(L, D)$. Theorem 3.6 of [7] then proves that the left $D$-module $E=D^{1 \times\left(p^{\prime}+2\right)} /\left(D^{1 \times p} Q\right)$, where $Q:=(P-\Lambda) \in D^{p \times\left(p^{\prime}+2\right)}$, is stably free of rank $p^{\prime}+2-p$. Since $\operatorname{rank}_{D}(L)=p^{\prime}-p \geq 0$, then $p^{\prime}+2-p \geq 2$, which shows that $E$ is a finitely generated free left $D$-module by Theorem 6. Finally, the result follows from Theorem 4.1 of [7].

Finally, if the right $D$-module $\operatorname{ext}_{D}^{1}(L, D)$ is cyclic, then there exists $\Lambda \in D^{q}$ such that $\operatorname{ext}_{D}^{1}(L, D)=\varpi(\Lambda) D$. Using Theorem 3.6 of [7], the left $D$-module $E^{\prime}=D^{1 \times\left(p^{\prime}+1\right)} /\left(D^{1 \times p} Q^{\prime}\right)$, where $Q^{\prime}:=\left(\begin{array}{ll}P & -\Lambda\end{array}\right) \in D^{p \times\left(p^{\prime}+1\right)}$, is stably free of rank $p^{\prime}+1-p$. If $p^{\prime}+1-p \geq 2$, i.e., $p^{\prime}-p \geq 1$, then $E^{\prime}$ is free by Theorem 6 and the result follows from Theorem 4.1 of [7].

Since holonomic modules over the standard rings of PD operators are cyclic (see, e.g., $[6,28])$, the second part of Corollary 3 yields Theorem 30 of [15].

Remark 9. Corollary 3 is most interesting in the case of a finitely generated left $D$-module $M$ satisfying $\operatorname{rank}_{D}(M) \geq 1$, i.e., for underdetermined linear systems. Indeed, if $P \in D^{p^{\prime} \times p^{\prime}}$ has full row rank, i.e., $\operatorname{rank}_{D}(M)=0$, then Corollary 3 yields again 1 of Corollary 2 .

Example 13. Since the global dimension of the rings $A_{1}(k), B_{1}(k), k \llbracket t \rrbracket\langle\partial\rangle$, and $k\{t\}\langle\partial\rangle$ where $k \in\{\mathbb{R}, \mathbb{C}\}$, is one (see, e.g., [6,28]), Section 3 of [36] shows that the first part of Corollary 3 holds for these rings. Hence, any underdetermined linear system of OD operators (namely, $\operatorname{rank}_{D}(M)=p^{\prime}-p \geq 1$ ) with either polynomial, rational, formal power series or locally convergent power series coefficients can be reduced to an equivalent linear OD system defined by at most two OD equations.

Following Stafford ([42]), let us now show how to use Corollary 2 to obtain the minimal number of generators of a finitely generated left $D$-module $L$ which is not torsion.

First of all, if $L$ can be generated by $r$ elements, then there exists a surjective left $D$ homomorphism $\omega: D^{1 \times r} \longrightarrow L$ defined by mapping the $i^{\text {th }}$ element of the standard basis of $D^{1 \times r}$ to the $i^{\text {th }}$ generator of $L$, i.e., we have the following short exact sequence:

$$
0 \longrightarrow \operatorname{ker} \omega \longrightarrow D^{1 \times r} \stackrel{\omega}{\longrightarrow} L \longrightarrow 0
$$

If $\operatorname{rank}_{D}(L)=r$, then the equality $\operatorname{rank}_{D}(L)+\operatorname{rank}_{D}(\operatorname{ker} \omega)=r$ yields $\operatorname{rank}_{D}(\operatorname{ker} \omega)=0$, i.e., $\operatorname{ker} \omega$ is either 0 or a torsion left $D$-module. But, $\operatorname{ker} \omega$ is a left $D$-submodule of the free, and thus of the torsion-free left $D$-module $D^{1 \times r}$, which yields $\operatorname{ker} \omega=0$, and proves that $L \cong D^{1 \times r}$, i.e., $L$ is a free left $D$-module of rank $r$. Conversely, if $L$ is a finitely generated free left $D$-module of rank $r$, then $L$ can be generated by $r$ elements. Hence, a finitely generated left $D$-module $L$ can be generated by $r:=\operatorname{rank}_{D}(L)$ elements if and only if $L \cong D^{1 \times r}$.

We note that a finitely generated left $D$-module $L$ cannot be generated by fewer elements than $\operatorname{rank}_{D}(L)$ since the short exact sequence (44) yields $r-\operatorname{rank}_{D}(L)=\operatorname{rank}_{D}(\operatorname{ker} \omega) \geq 0$.

Now, let us suppose that $L$ is not free, i.e., cannot be generated by $r:=\operatorname{rank}_{D}(L)$ elements. By Theorem 7, there exists a left $D$-module $L^{\prime}$ with $\operatorname{rank}_{D}\left(L^{\prime}\right) \leq 1$ such that 
$L \cong D^{1 \times s} \oplus L^{\prime}$. If $\operatorname{rank}_{D}\left(L^{\prime}\right)=0$, i.e., $L^{\prime}$ is a torsion left $D$-module, then $r=s$, which yields $L \cong D^{1 \times(r-1)} \oplus\left(D \oplus L^{\prime}\right)$, where $\operatorname{rank}_{D}\left(D \oplus L^{\prime}\right)=1$. Now, if $\operatorname{rank}_{D}\left(L^{\prime}\right)=1$, then $s=r-1$. Hence, with a change of notations, we can always suppose that $L \cong D^{1 \times(r-1)} \oplus L^{\prime}$, where $\operatorname{rank}_{D}\left(L^{\prime}\right)=1$. Since $L^{\prime}$ is not a torsion left $D$-module, there exists $l^{\prime} \in L^{\prime} \backslash t\left(L^{\prime}\right)$. Thus, $\operatorname{ann}_{D}\left(l^{\prime}\right)=\left\{d \in D \mid d l^{\prime}=0\right\}=0$, i.e., $D l^{\prime} \cong D / \operatorname{ann}_{D}\left(l^{\prime}\right) \cong D$. Now, the following short exact sequence

$$
0 \longrightarrow D l^{\prime} \longrightarrow L^{\prime} \longrightarrow L^{\prime} /\left(D l^{\prime}\right) \longrightarrow 0
$$

yields $\operatorname{rank}_{D}\left(L^{\prime} /\left(D l^{\prime}\right)\right)=0$, i.e., $L^{\prime} /\left(D l^{\prime}\right)$ is a torsion left $D$-module. Now, 2 of Corollary 2 shows that there exist a projective left ideal $I$ of $D$ and a left subideal $J$ of $I$ such that $L^{\prime} /\left(D l^{\prime}\right) \cong I / J$. Thus, we get the short exact sequence $0 \longrightarrow D l^{\prime} \stackrel{\alpha}{\longrightarrow} L^{\prime} \stackrel{\beta}{\longrightarrow} I / J \longrightarrow 0$. Hence, the following diagram has an exact row and exact columns

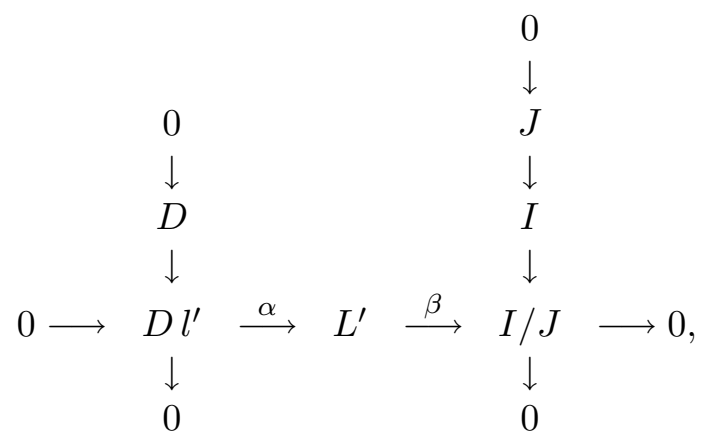

which can be completed to the following commutative diagram with exact rows and columns:

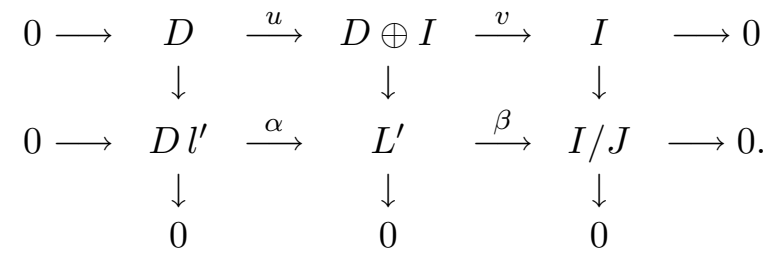

For more details, see, e.g., [38]. We now note that $D \oplus I$ is a projective left $D$-module with $\operatorname{rank}_{D}(D \oplus I)=2$, and thus $D \oplus I$ is a free left $D$-module of rank two by Theorem 6, i.e., $D \oplus I \cong D^{1 \times 2}$. Therefore, $L^{\prime}$ can be defined by two generators (but no fewer), which finally shows that $L$ can be generated by $r-1+2=r+1$ elements but no fewer.

Lemma 5. Let $M$ be a non-free left $D$-module with $\operatorname{rank}_{D}(M)=1$. Then, $M$ can be generated by two elements and no fewer.

Let us explain how to get a presentation of $L^{\prime}=D^{1 \times r} /\left(D^{1 \times s} Q\right)$ defined by only two generators. Since $\operatorname{rank}_{D}\left(L^{\prime}\right)=1, \operatorname{rank}_{D}\left(D^{1 \times s} Q^{\prime}\right)=r-1$. If $r-1=1$, i.e., $r=2$, then nothing has to be done. Let us now suppose that $r-1 \geq 2$, i.e., $r \geq 3$. Then, applying Algorithm 3, we get $L^{\prime} \cong L^{\prime \prime}=D^{1 \times(r-1)} /\left(D^{1 \times t} Q^{\prime}\right)$ where $\operatorname{rank}_{D}\left(D^{1 \times t} Q^{\prime}\right)=r-2$. Repeating the same argument, we finally get $L^{\prime} \cong \bar{L}=D^{1 \times 2} /\left(D^{1 \times l} \bar{Q}\right)$. 
Example 14. Using standard results on the minimal number of generators of finitely presented modules over a commutative ring based on Fitting ideals (see, e.g., [19]), we can easily check that the $E=\mathbb{Q}\left[\partial_{1}, \partial_{2}, \partial_{3}\right]$-module $L=E^{1 \times 3} /\left(E^{1 \times 3} R\right)$, where $R$ is the matrix (20) defining the curl operator in $\mathbb{R}^{3}$ (see Example 8), can be generated by three elements but no fewer. Now, if we consider the left $D=A_{3}(\mathbb{Q})$-module $M=D^{1 \times 3} /\left(D^{1 \times 3} R\right) \cong D \otimes_{E} L$, then $\operatorname{rank}_{D}(M)=1$ and Lemma 5 shows that $M$ can be generated by two elements but no fewer. If $\left\{f_{j}\right\}_{j=1,2,3}$ is the standard basis of $D^{1 \times 3}, \pi: D^{1 \times 3} \longrightarrow M$ the canonical projection onto $M$, $\left\{y_{j}=\pi\left(f_{j}\right)\right\}_{j=1,2,3}$ a family of generators of $M$, and $y=\left(\begin{array}{lll}y_{1} & y_{2} & y_{3}\end{array}\right)^{T}$, then it was shown in Example 8 that $\left\{z_{1}=y_{1}, z_{2}=\left(x_{1}+1\right) y_{2}+y_{3}\right\}$ is also a family of generators of $M$ and $y=U z$, where the matrix $U$ is defined by (36) and $z=\left(\begin{array}{ll}z_{1} & z_{2}\end{array}\right)^{T}$.

Let us sum up the above remarks in the next theorem due to Stafford.

Theorem 10 ([42]). Let $L$ be a finitely generated left D-module that is not a torsion module, i.e., $\operatorname{rank}_{D}(L) \geq 1$. Then, the following statements are equivalent:

1. $\operatorname{rank}_{D}(L)=r$.

2. Either $L$ is a free left $D$-module of rank $r$, i.e., $L \cong D^{1 \times r}$, and thus $L$ can be generated by $r$ elements, or $L$ is not a free left $D$-module and $L$ can be generated by $r+1$ elements but no fewer.

Example 15. If $M=D^{1 \times 3} /(D R)$ is the left $D=A_{3}(\mathbb{Q})$-module finitely presented by the divergence operator $R$, then it is well-known that $M$ is torsion-free but not free (see $[11,36]$ ). Since $\operatorname{rank}_{D}(M)=2$, by Theorem 10, $M$ can be generated by three elements. Hence, $R$ is a minimal presentation of $M$, namely, a presentation of $M$ having the minimal number of generators $\mu(M)$ of $M$.

Remark 10. Corollary 2 shows that a finitely generated torsion left $D$-module $M$ is either cyclic, i.e., generated by one element, or can be generated by two elements. Theorem 10 (see also Lemma 5) shows that a finitely generated left $D$-module of rank one is either free, i.e., cyclic, or can be generated by two elements and no fewer. Hence, a finitely generated left $D$-module $M$ satisfying $\operatorname{rank}_{D}(M) \leq 1$ can always be generated by one or two elements. From a systems theory viewpoint, Remark 5 and the above results show that the study of $\operatorname{ext}_{D}^{i}(M, \mathcal{F})$ for $i \geq 0\left(\operatorname{ext}_{D}^{0}(M, \mathcal{F})=\operatorname{hom}_{D}(M, \mathcal{F})\right)$ can be reduced to $\operatorname{ext}_{D}^{i}\left(M^{\prime}, \mathcal{F}\right)$ for $i \geq 0$, where $\operatorname{rank}_{D}\left(M^{\prime}\right) \leq 1$, and thus to the case of a left $D$-module $M^{\prime}$ which is either zero, can be generated by one or by two elements. Finally, Theorem 10 also shows that a finitely generated left $D$-module $M$ which cannot be generated by two elements satisfies $\operatorname{rank}_{D}(M) \geq 2$.

\section{Cancellation theorem}

\subsection{Main result}

Let us state a result due to Stafford (which can be traced back to Swan [43]).

$\mathrm{RR} \mathrm{n}^{\circ} 8225$ 
Lemma 6 ([42]). Let $D$ be a very simple domain satisfying (8), $M$ a finitely generated left $D$-module with $\operatorname{rank}_{D}(M) \geq 2, d^{\star} \in D, m^{\star} \in M$ such that $\left(d^{\star}, m^{\star}\right)$ is a unimodular element of $D \oplus M$. Then, there exists $\phi \in \operatorname{hom}_{D}(D, M)$ such that:

$$
\phi\left(d^{\star}\right)+m^{\star} \in \mathrm{U}(M) .
$$

Proof. Since $\left(d^{\star}, m^{\star}\right) \in \mathrm{U}(D \oplus M)$, there exist $\omega_{1} \in \operatorname{hom}_{D}(D, D)$ and $\omega_{2} \in \operatorname{hom}_{D}(M, D)$ such that $\omega=\omega_{1} \oplus \omega_{2} \in \operatorname{hom}_{D}(D \oplus M, D)$ satisfies:

$$
\omega\left(\left(d^{\star}, m^{\star}\right)\right)=\omega_{1}\left(d^{\star}\right)+\omega_{2}\left(m^{\star}\right)=1 .
$$

Let $R \in D^{q \times p}$ be such that $M=D^{1 \times p} /\left(D^{1 \times q} R\right)$ and $\pi: D^{1 \times p} \longrightarrow M$ the canonical projection onto $M$. Since $\operatorname{rank}_{D}(M) \geq 2$, applying the first seven steps of Algorithm 1, there exist two elements $m_{1}=\pi\left(\lambda_{1}\right)$ and $m_{2}=\pi\left(\lambda_{2}\right)$ of $M$, where $\lambda_{1}, \lambda_{2} \in D^{1 \times p}$, and two elements $\mu_{1}$ and $\mu_{2}$ of $D^{p}$ such that $\varphi_{i}:=\varphi_{\mu_{i}} \in \operatorname{hom}_{D}(M, D), i=1,2$, satisfy:

$$
\varphi_{1}\left(m_{1}\right) \neq 0, \quad \varphi_{1}\left(m_{2}\right)=0, \quad \varphi_{2}\left(m_{1}\right)=0, \quad \varphi_{2}\left(m_{2}\right) \neq 0 .
$$

Using (8), there exist $r, s \in D$ such that:

$$
\varphi_{1}\left(m^{\star}\right) D+\varphi_{2}\left(m^{\star}\right) D+d^{\star} D=\left(\varphi_{1}\left(m^{\star}\right)+d^{\star} r \varphi_{1}\left(m_{1}\right)\right) D+\left(\varphi_{2}\left(m^{\star}\right)+d^{\star} s \varphi_{2}\left(m_{2}\right)\right) D .
$$

In particular, there exist $\alpha, \beta \in D$ such that:

$$
d^{\star}=\left(\varphi_{1}\left(m^{\star}\right)+d^{\star} r \varphi_{1}\left(m_{1}\right)\right) \alpha+\left(\varphi_{2}\left(m^{\star}\right)+d^{\star} s \varphi_{2}\left(m_{2}\right)\right) \beta .
$$

Using the right $D$-module structure of $\operatorname{hom}_{D}(M, D)$, let $\chi:=\varphi_{1} \alpha+\varphi_{2} \beta \in \operatorname{hom}_{D}(M, D)$ be defined by $\chi(m)=\varphi_{1}(m) \alpha+\varphi_{2}(m) \beta$ for all $m \in M$. Let us also consider $\phi \in \operatorname{hom}_{D}(D, M)$ defined by $\phi(d)=d\left(r m_{1}+s m_{2}\right)$ for all $d \in D$. Then, using (46), we get

$$
\begin{aligned}
\chi\left(\phi\left(d^{\star}\right)\right) & =\chi\left(d^{\star} \phi(1)\right)=d^{\star} \chi\left(r m_{1}+s m_{2}\right) \\
& =d^{\star}\left(\varphi_{1}\left(r m_{1}+s m_{2}\right) \alpha+\varphi_{2}\left(r m_{1}+s m_{2}\right) \beta\right) \\
& =d^{\star}\left(r \varphi_{1}\left(m_{1}\right) \alpha+s \varphi_{2}\left(m_{2}\right) \beta\right)
\end{aligned}
$$

which combined with (48) yields:

$$
\begin{aligned}
\chi\left(m^{\star}+\phi\left(d^{\star}\right)\right) & =\varphi_{1}\left(m^{\star}\right) \alpha+\varphi_{2}\left(m^{\star}\right) \beta+d^{\star}\left(r \varphi_{1}\left(m_{1}\right) \alpha+s \varphi_{2}\left(m_{2}\right) \beta\right) \\
& =\left(\varphi_{1}\left(m^{\star}\right)+d^{\star} r \varphi_{1}\left(m_{1}\right)\right) \alpha+\left(\varphi_{2}\left(m^{\star}\right)+d^{\star} s \varphi_{2}\left(m_{2}\right)\right) \beta=d^{\star} .
\end{aligned}
$$

Let us define $\psi:=\left(\omega_{1}-\omega_{2} \circ \phi\right) \oplus \omega_{2} \in \operatorname{hom}_{D}(D \oplus M, D)$. Then, using (45), we get

$$
\psi\left(\left(d^{\star}, m^{\star}+\phi\left(d^{\star}\right)\right)\right)=\omega_{1}\left(d^{\star}\right)-\omega_{2}\left(\phi\left(d^{\star}\right)\right)+\omega_{2}\left(m^{\star}+\phi\left(d^{\star}\right)\right)=\omega_{1}\left(d^{\star}\right)+\omega_{2}\left(m^{\star}\right)=1,
$$

$\mathrm{RR} \mathrm{n}^{\circ} 8225$ 
which shows that $\left(d^{\star}, m^{\star}+\phi\left(d^{\star}\right)\right) \in \mathrm{U}(D \oplus M)$. Let us define $t:=\omega_{1}(1)-\omega_{2}(\phi(1)) \in D$ and $\varphi:=\omega_{2}+\chi t \in \operatorname{hom}_{D}(M, D)$. Thus, using (45) and (49), we obtain

$$
\begin{aligned}
\varphi\left(m^{\star}+\phi\left(d^{\star}\right)\right) & =\left(\omega_{2}+\chi t\right)\left(m^{\star}+\phi\left(d^{\star}\right)\right) \\
& =\omega_{2}\left(m^{\star}\right)+\omega_{2}\left(\phi\left(d^{\star}\right)\right)+\chi\left(m^{\star}+\phi\left(d^{\star}\right)\right) t \\
& =1-\omega_{1}\left(d^{\star}\right)+\omega_{2}\left(d^{\star} \phi(1)\right)+d^{\star} t \\
& =1-d^{\star} \omega_{1}(1)+d^{\star} \omega_{2}(\phi(1))+d^{\star} t \\
& =1-d^{\star} t+d^{\star} t=1,
\end{aligned}
$$

which finally shows that $\widetilde{m}:=m^{\star}+\phi\left(d^{\star}\right) \in \mathrm{U}(M)$ and $\varphi(\widetilde{m})=1$.

If $D$ admits an involution, then (8) is a direct consequence of (6) as noticed in Remark 3.

Note that if $d^{\star}=0$, then $m^{\star} \in \mathrm{U}(M)$ and we can choose $\phi=\operatorname{id}_{D}$.

Remark 11. If $m^{\star} \notin t(M)$, then we can choose $m_{1}=m^{\star}$ so that $\varphi_{2}\left(m_{1}\right)=\varphi_{2}\left(m^{\star}\right)=0$ and (47) simplifies to $\varphi_{1}\left(m^{\star}\right) D+d^{\star} D=\left(1+d^{\star} r\right) \varphi_{1}\left(m^{\star}\right) D+d^{\star} s \varphi_{2}\left(m_{2}\right) D$, i.e., we need to find $r, s, \alpha, \beta \in D$ such that $d^{\star}=\left(1+d^{\star} r\right) \varphi_{1}\left(m^{\star}\right) \alpha+d^{\star} s \varphi_{2}\left(m_{2}\right) \beta$.

Remark 12. If $\operatorname{rank}_{D}(M) \geq 2$ and $m \in M$, then considering $\omega_{1}=\operatorname{id}_{D}$ and $\omega_{2}=0$, then $(1, m) \in \mathrm{U}(D \oplus M)$. Lemma 6 then shows that there exists $\phi \in \operatorname{hom}_{D}(D, M)$ such that $m^{\prime}:=\phi(1)+m \in \mathrm{U}(M)$, and thus $M=D m^{\prime} \oplus M^{\prime}$ for a certain left $D$-module $M^{\prime}$, which proves again Theorem 7 . If $m \notin t(M)$, then using Remark 11 with $d^{\star}=1$, the unimodular element $m^{\prime}$ can be obtained by computing a solution of the following inhomogeneous quadratic equation:

$$
1=(1+r) \varphi_{1}\left(m_{1}\right) \alpha+s \varphi_{2}\left(m_{2}\right) \beta .
$$

We find again the main argument of the proof of Theorem 7 .

\section{Algorithm 5 (ReductionOfUnimodularElement).}

- Input: A very simple domain $D$ satisfying (8), $R \in D^{q \times p}$ a matrix such that the left $D$-module $M=D^{1 \times p} /\left(D^{1 \times q} R\right)$ has rank at least two, i.e., $\operatorname{rank}_{D}(M) \geq 2, d^{\star} \in D$, $\lambda^{\star} \in D^{1 \times p}$ such that $\left(d^{\star}, m^{\star}\right) \in \mathrm{U}(D \oplus M)$, where $m^{\star}=\pi\left(\lambda^{\star}\right)$ and $\pi: D^{1 \times p} \longrightarrow M$ is the canonical projection, and $e \in D$ and $\mu \in \operatorname{ker}_{D}(R$. $)$ such that $\omega \in \operatorname{hom}_{D}(D \oplus M, D)$ defined by

$$
\forall d \in D, \quad \forall \lambda \in D^{1 \times p}, \quad \omega((d, \pi(\lambda)))=d e+\lambda \mu,
$$

satisfies $\omega\left(\left(d^{\star}, m^{\star}\right)\right)=1$, i.e., $d^{\star} e+\lambda^{\star} \mu=1$.

\section{- Output:}

1. $\bar{\lambda} \in D^{1 \times p}$ such that $\phi \in \operatorname{hom}_{D}(D, M)$ defined by $\phi(d)=d \bar{m}$ for all $d \in D$, where $\bar{m}:=\pi(\bar{\lambda})$, satisfies $m^{\star}+\phi\left(d^{\star}\right)=m^{\star}+d^{\star} \bar{m} \in \mathrm{U}(M)$. 
2. $\bar{\mu} \in \operatorname{ker}_{D}(R$.$) such that \varphi:=\varphi_{\bar{\mu}} \in \operatorname{hom}_{D}(M, D)$ satisfies $\varphi\left(m^{\star}+\phi\left(d^{\star}\right)\right)=1$, i.e.:

$$
\left(\lambda^{\star}+d^{\star} \bar{\lambda}\right) \bar{\mu}=1 \text {. }
$$

1. Applying steps 1-7 of UnimodularElement (see Algorithm 1) to $R$, we get $\lambda_{1}, \lambda_{2} \in D^{1 \times p}$ and $\mu_{1}, \mu_{2} \in \operatorname{ker}_{D}(R$. $)$ such that $d_{1}:=\varphi_{1}\left(m_{1}\right) \neq 0, \varphi_{1}\left(m_{2}\right)=0, d_{2}:=\varphi_{2}\left(m_{2}\right) \neq 0$, and $\varphi_{2}\left(m_{1}\right)=0$, where $\varphi_{i}:=\varphi_{\mu_{i}} \in \operatorname{hom}_{D}(M, D)$, and $m_{i}=\pi\left(\lambda_{i}\right)$ for $i=1,2$. Note that if $\pi(\lambda) \notin t(M)$, then we can take $\lambda_{1}:=\lambda$.

2. Using (8), compute $r, s \in D$ such that:

$$
\left(\lambda^{\star} \mu_{1}\right) D+\left(\lambda^{\star} \mu_{2}\right) D+d^{\star} D=\left(\lambda^{\star} \mu_{1}+d^{\star} r \lambda_{1} \mu_{1}\right) D+\left(\lambda^{\star} \mu_{2}+d^{\star} s \lambda_{2} \mu_{2}\right) D .
$$

3. Define $\bar{\lambda}:=r \lambda_{1}+s \lambda_{2}$.

4. Right factorize $d^{\star}$ by $S=\left(\left(\lambda^{\star}+d^{\star} r \lambda_{1}\right) \mu_{1} \quad\left(\lambda^{\star}+d^{\star} s \lambda_{2}\right) \mu_{2}\right)$ to get $d^{\star}=S\left(\begin{array}{ll}\alpha & \beta\end{array}\right)^{T}$, where $\alpha, \beta \in D$.

5. Define $\mu^{\star}:=\mu_{1} \alpha+\mu_{2} \beta \in \operatorname{ker}_{D}(R), t:.=e-\bar{\lambda} \mu$, and $\bar{\mu}:=\mu+\mu^{\star} t \in \operatorname{ker}_{D}(R$.).

6. Return $\bar{\lambda}$ and $\bar{\mu}$.

Let us check (50). Using $\lambda_{1} \mu_{2}=0, \lambda_{2} \mu_{1}=0$, and 4 of Algorithm 5, we first get:

$$
\begin{aligned}
\left(\lambda^{\star}+d^{\star}\left(r \lambda_{1}+s \lambda_{2}\right)\right)\left(\mu_{1} \alpha+\mu_{2} \beta\right) & =\lambda^{\star}\left(\mu_{1} \alpha+\mu_{2} \beta\right)+d^{\star}\left(r \lambda_{1}+s \lambda_{2}\right)\left(\mu_{1} \alpha+\mu_{2} \beta\right) \\
& =\lambda^{\star}\left(\mu_{1} \alpha+\mu_{2} \beta\right)+d^{\star}\left(r \lambda_{1} \mu_{1} \alpha+s \lambda_{2} \mu_{2} \beta\right) \\
& =\left(\lambda^{\star}+d^{\star} r \lambda_{1}\right) \mu_{1} \alpha+\left(\lambda^{\star}+d^{\star} s \lambda_{2}\right) \mu_{2} \beta=d^{\star} .
\end{aligned}
$$

With the notations of 5 of Algorithm 5, using $\bar{\mu}=\mu+\left(\mu_{1} \alpha+\mu_{2} \beta\right) t, t=e-\left(r \lambda_{1}+s \lambda_{2}\right) \mu$, $d^{\star} e+\lambda^{\star} \mu=1$, and the above identity, it follows:

$$
\begin{aligned}
\left(\lambda^{\star}+d^{\star} \bar{\lambda}\right) \bar{\mu} & =\left(\lambda^{\star}+d^{\star}\left(r \lambda_{1}+s \lambda_{2}\right)\right)\left(\mu+\left(\mu_{1} \alpha+\mu_{2} \beta\right) t\right) \\
& =\lambda^{\star} \mu+d^{\star}\left(r \lambda_{1}+s \lambda_{2}\right) \mu+\left(\lambda^{\star}+d^{\star}\left(r \lambda_{1}+s \lambda_{2}\right)\right)\left(\mu_{1} \alpha+\mu_{2} \beta\right) t \\
& =1-d^{\star} e+d^{\star}\left(r \lambda_{1}+s \lambda_{2}\right) \mu+d^{\star} t \\
& =1-d^{\star}\left(e-\left(r \lambda_{1}+s \lambda_{2}\right) \mu\right)+d^{\star} t=1 .
\end{aligned}
$$

Remark 13. If $M=D^{1 \times r}$ with $r \geq 2$, then Theorem 6 and Algorithm 5 show that for every $\left(\begin{array}{ll}d^{\star} & \lambda^{\star}\end{array}\right) \in \mathrm{U}\left(D^{1 \times(1+r)}\right)$, where $d^{\star} \in D$ and $\lambda^{\star} \in D^{1 \times r}$, i.e., such that $\left(\begin{array}{ll}d^{\star} & \lambda^{\star}\end{array}\right)$ admits a right inverse over $D$, there exists $\bar{\lambda} \in D^{1 \times r}$ such that $\widetilde{\lambda}:=\lambda+d^{\star} \bar{\lambda} \in \mathrm{U}\left(D^{1 \times r}\right)$, i.e., such that $\widetilde{\lambda}$ admits a right inverse over $D$. This result comes from the fact that the stable rank of $D$ is at most 2, i.e., $\operatorname{sr}(D) \leq 2$, which is a direct consequence of (6) as explained in [36].

$\mathrm{RR} \mathrm{n}^{\circ} 8225$ 
Example 16. Let us consider again Example 5. Let $d^{\star}=\partial_{1}, \lambda^{\star}=\left(\begin{array}{llll}0 & 0 & -x_{1}\end{array}\right)$, and $m^{\star}=\pi\left(\lambda^{\star}\right)$. If $e=x_{1}$ and $\mu=\left(\begin{array}{lll}-\partial_{3} & 0 & \partial_{1}\end{array}\right)^{T} \in \operatorname{ker}_{D}(R$. $)$, then $d^{\star} e+\lambda^{\star} \mu=1$, which implies that $d^{\star} \oplus m^{\star} \in \mathrm{U}(D \oplus M)$ since $\varphi \in \operatorname{hom}_{D}(D \oplus M, D)$ defined by $\varphi(d \oplus \pi(\lambda))=d e+\lambda \mu$ for all $d \in D$ and for all $\lambda \in D^{1 \times 3}$ satisfies $\varphi\left(d^{\star} \oplus m^{\star}\right)=d^{\star} e+\lambda^{\star} \mu=1$. By Lemma 6 , there exists $\bar{\lambda} \in D^{1 \times 3}$ such that $\phi \in \operatorname{hom}_{D}(D, M)$ defined by $\phi(d)=d \pi(\bar{\lambda})$ for all $d \in D$ satisfies that $m^{\star}+\phi\left(d^{\star}\right)=\pi\left(\lambda^{\star}+d^{\star} \bar{\lambda}\right) \in \mathrm{U}(M)$. Using Algorithm 5, let us compute $\bar{\lambda}$.

Let $\lambda_{1}=\left(\begin{array}{lll}0 & -1 & 0\end{array}\right), \lambda_{2}=\left(\begin{array}{lll}0 & 0 & 1\end{array}\right), \mu_{1}=\left(\begin{array}{lll}\partial_{2} & -\partial_{1} & 0\end{array}\right)^{T}$, and $\mu_{2}=\left(\begin{array}{lll}-\partial_{3} & 0 & \partial_{1}\end{array}\right)^{T}$. Then, using Example 5, we have $d_{1}:=\varphi_{1}\left(m_{1}\right)=\partial_{1}, \varphi_{1}\left(m_{2}\right)=0, d_{2}:=\varphi_{2}\left(m_{2}\right)=\partial_{1}$, and $\varphi_{2}\left(m_{1}\right)=0$, where $\varphi_{i}:=\varphi_{\mu_{i}} \in \operatorname{hom}_{D}(M, D)$, and $m_{i}=\pi\left(\lambda_{i}\right)$ for $i=1$, 2. Then, $d_{3}:=\varphi_{1}\left(m^{\star}\right)=\lambda^{\star} \mu_{1}=0$ and $d_{4}:=\varphi_{2}\left(m^{\star}\right)=\lambda^{\star} \mu_{2}=-x_{1} \partial_{1}$. Using Remark 3, we can compute $r, s \in D$ satisfying (47) by computing $u, v \in D$ such that

$$
D \theta\left(d_{3}\right)+D \theta\left(d_{4}\right)+D \theta\left(d^{\star}\right)=D\left(\theta\left(d_{3}\right)+\theta\left(d_{1}\right) u \theta\left(d^{\star}\right)\right)+D\left(\theta\left(d_{4}\right)+\theta\left(d_{2}\right) v \theta\left(d^{\star}\right)\right),
$$

where $\theta\left(d_{3}\right)=0, \theta\left(d_{4}\right)=x_{1} \partial_{1}+1$, and $\theta\left(d^{\star}\right)=-\partial_{1}$, and then defining $r=\theta(u)$ and $s=\theta(v)$. We can take $u=1$ and $v=0$, and thus $r=1$ and $s=0$. Then, we obtain $\bar{\lambda}=\lambda_{1}$ and $\Lambda:=\lambda^{\star}+d^{\star} \bar{\lambda}=\left(\begin{array}{lll}0 & -\partial_{1} & -x_{1}\end{array}\right)$ is such that $\pi(\Lambda) \in \mathrm{U}(M)$.

Let us compute $\bar{\mu} \in \operatorname{ker}_{D}(R$. $)$ such that $\Lambda \bar{\mu}=1$, i.e., such that $\varphi \in \operatorname{hom}_{D}(M, D)$ defined by $\varphi(\pi(\lambda))=\lambda \bar{\mu}$ for all $\lambda \in D^{1 \times 3}$ satisfies $\varphi(\pi(\Lambda))=1$. Right factoring $d^{\star}=\partial_{1}$ by the matrix $S=\left(\begin{array}{ll}\partial_{1}^{2} & -x_{1} \partial_{1}\end{array}\right)$, we get $d^{\star}=S\left(\begin{array}{ll}\alpha & \beta\end{array}\right)^{T}$, where $\alpha=\frac{1}{2} x_{1}$ and $\beta=\frac{1}{2} \partial_{1}$, which yields $\mu^{\star}=\frac{1}{2}\left(\begin{array}{lll}-\partial_{1} \partial_{3}+x_{1} \partial_{2} & -x_{1} \partial_{1}-1 \quad \partial_{1}^{2}\end{array}\right)^{T}, t=x_{1}$, and:

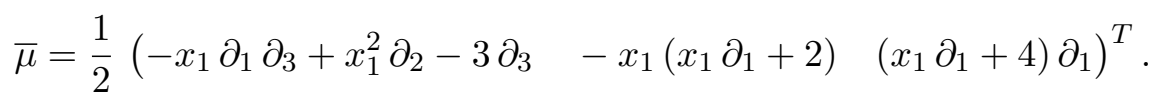

Finally, we note that we can also use Remark 2 to compute $\bar{\mu}$.

Lemma 6 is the key result for the so-called (Bass') cancellation theorem ([42]) which states that $M \oplus D \cong N \oplus D$ implies $M \cong N$ when $\operatorname{rank}_{D}(M) \geq 2$. Let us explicitly describe this last isomorphism following the proof of Corollary 12.6 of [43] as pointed out in [42].

Let $f: D \oplus M \longrightarrow D \oplus N$ be a left $D$-isomorphism and $\left(d^{\star}, n^{\star}\right):=f((1,0))$. Now, if $g: D \oplus M \longrightarrow D$ is defined by $g((d, m))=d$ for all $d \in D$ and for all $m \in M$, then $\left(g \circ f^{-1}\right)\left(\left(d^{\star}, n^{\star}\right)\right)=g((1,0))=1$, which shows that $\left(d^{\star}, n^{\star}\right) \in \mathrm{U}(D \oplus N)$. By Lemma 6 , there exists $\phi \in \operatorname{hom}_{D}(D, N)$ such that $\widetilde{n}:=n^{\star}+\phi\left(d^{\star}\right) \in \mathrm{U}(N)$. Thus, there exists $\varphi \in \operatorname{hom}_{D}(N, D)$ such that $\varphi(\widetilde{n})=1$. Now, let us consider the following left $D$-homomorphisms:

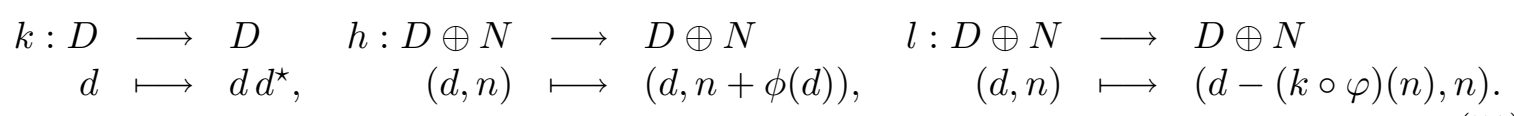

Since $h$ and $l$ are automorphisms of $D \oplus N, i:=l \circ h \circ f: D \oplus M \longrightarrow D \oplus N$ is a left $D$ isomorphism. Moreover, $i((1,0))=(l \circ h)\left(\left(d^{\star}, n^{\star}\right)\right)=l\left(\left(d^{\star}, \widetilde{n}\right)\right)=\left(d^{\star}-(k \circ \varphi)(\widetilde{n}), \widetilde{n}\right)=(0, \widetilde{n})$. 
Now, $N=D \tilde{n} \oplus \operatorname{ker} \varphi \cong D \oplus \operatorname{ker} \varphi$ since $\widetilde{n} \in \mathrm{U}(N)$. If $X:=D \oplus N=D \oplus(D \tilde{n} \oplus \operatorname{ker} \varphi)$, then $Y:=X /(0 \oplus D \widetilde{n}) \cong D \oplus \operatorname{ker} \varphi \cong D \widetilde{n} \oplus \operatorname{ker} \varphi=N$. Using (4), we have the direct sum decomposition

$$
\forall n \in N, \quad n=\varphi(n) \widetilde{n}+(n-\varphi(n) \widetilde{n}), \quad \varphi(n) \tilde{n} \in D \widetilde{n}, \quad n-\varphi(n) \widetilde{n} \in \operatorname{ker} \varphi,
$$

and the left $D$-isomorphism $N \cong Y$ is then defined by

$$
\begin{aligned}
& \varepsilon: N \longrightarrow Y \quad \varepsilon^{-1}: Y \longrightarrow N \\
& n \longmapsto \sigma_{1}((\varphi(n), n)), \quad \sigma_{1}((d, n)) \longmapsto n+(d-\varphi(n)) \tilde{n},
\end{aligned}
$$

where $\sigma_{1}: X=D \oplus N \longrightarrow Y$ is the canonical projection onto $Y$.

If $i_{1} \in \operatorname{hom}_{D}(D, D \oplus N)$ (resp., $i_{2} \in \operatorname{hom}_{D}(D, D \oplus M)$ ) is defined by $i_{1}(1)=(0, \widetilde{n})$ (resp., $\left.i_{2}(1)=(1,0)\right)$, then $\operatorname{im} i_{1}=D(0, \widetilde{n})$ and $\operatorname{im} i_{2}=D(1,0)$, which yields

$$
\text { coker } i_{1}=Y, \quad Z:=\text { coker } i_{2}=(D \oplus M) /(D(1,0)) \cong M,
$$

where the last left $D$-isomorphism is defined by

$$
\begin{aligned}
\kappa: M & \longrightarrow Z \\
m & \longmapsto \sigma_{2}((0, m)),
\end{aligned}
$$

where $\sigma_{2}: D \oplus M \longrightarrow Z$ is the canonical projection onto $Z$.

Using $i \circ i_{2}=i_{1}$, the following diagram is commutative with exact rows and columns

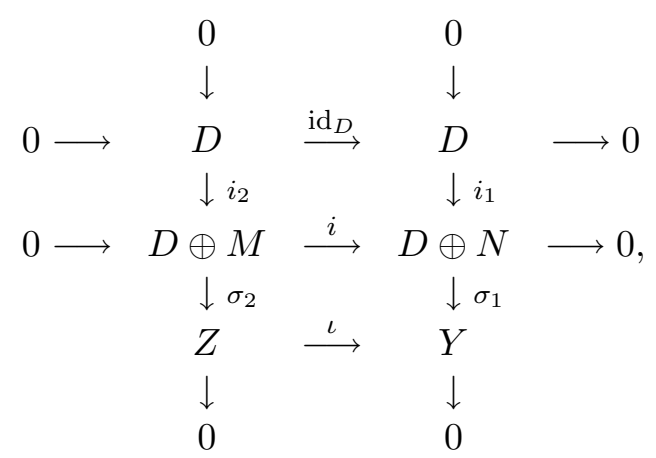

where $\iota\left(\sigma_{2}((d, m))\right)=\sigma_{1}(i((d, m)))$ for all $d \in D$ and for all $m \in M$. With the notation $(e, n):=i((0, m))$, the left $D$-isomorphism $\varpi:=\varepsilon^{-1} \circ \iota \circ \kappa \in \operatorname{hom}_{D}(M, N)$ is defined by:

$$
\begin{aligned}
\varpi: M & \longrightarrow N \\
m & \longmapsto n+(e-\varphi(n)) \widetilde{n} .
\end{aligned}
$$

Theorem 11 ([42]). Let $D$ be a very simple domain satisfying (8), $M$ and $N$ two finitely generated left $D$-modules, and $\operatorname{rank}_{D}(M) \geq 2$. Then, $M \oplus D \cong N \oplus D$ implies $M \cong N$.

$\operatorname{RR} n^{\circ} 8225$ 
A similar result holds for right $D$-modules.

Let us give an explicit description of $\varpi$. Let $M=D^{1 \times p} /\left(D^{1 \times q} R\right), N=D^{1 \times p^{\prime}} /\left(D^{1 \times q^{\prime}} R^{\prime}\right)$, $\pi: D^{1 \times p} \longrightarrow M$ (resp., $\pi^{\prime}: D^{1 \times p^{\prime}} \longrightarrow N$ ) be the canonical projection onto $M$ (resp., $N$ ), $P=\left(\begin{array}{ll}0 & R\end{array}\right) \in D^{q \times(1+p)}, P^{\prime}=\left(\begin{array}{ll}0 & R^{\prime}\end{array}\right) \in D^{q^{\prime} \times\left(1+p^{\prime}\right)}, L=D^{1 \times(1+p)} /\left(D^{1 \times q} P\right) \cong D \oplus M$, $L^{\prime}=D^{1 \times\left(1+p^{\prime}\right)} /\left(D^{1 \times q^{\prime}} P^{\prime}\right) \cong D \oplus N$, and $\tau: D^{1 \times(1+p)} \longrightarrow L\left(\right.$ resp., $\left.\tau^{\prime}: D^{1 \times\left(1+p^{\prime}\right)} \longrightarrow L^{\prime}\right)$ the canonical projection onto $L$ (resp., $L^{\prime}$ ). Let $f$ be a left $D$-isomorphism defined by

$$
\begin{aligned}
f: L & \longrightarrow L^{\prime} \\
\tau(\zeta) & \longmapsto \tau^{\prime}(\zeta X),
\end{aligned}
$$

where:

$$
X=\left(\begin{array}{cc}
X_{11} & X_{12} \\
X_{21} & X_{22}
\end{array}\right) \in D^{(1+p) \times\left(1+p^{\prime}\right)}, \quad X_{11} \in D, X_{12} \in D^{1 \times p^{\prime}}, X_{21} \in D^{p}, X_{22} \in D^{p \times p^{\prime}}
$$

Then, $f(\tau(1 \quad 0))=\tau^{\prime}\left(\left(\begin{array}{ll}X_{11} & X_{12}\end{array}\right)\right)$. Now, applying Lemma 6 , there exist $\bar{\lambda} \in D^{1 \times p^{\prime}}$ and $\bar{\mu} \in \operatorname{ker}_{D}\left(R^{\prime}\right.$.) such that $\left(X_{12}+X_{11} \bar{\lambda}\right) \bar{\mu}=1$. The left $D$-isomorphism $i$ is then defined by

$$
\begin{aligned}
& i: L \quad \longrightarrow L^{\prime} \\
& \tau(\zeta) \quad \longmapsto \tau^{\prime}\left(\zeta X^{\prime}\right),
\end{aligned}
$$

where:

$$
\begin{aligned}
X^{\prime}=\left(\begin{array}{cc}
X_{11}^{\prime} & X_{12}^{\prime} \\
X_{21}^{\prime} & X_{22}^{\prime}
\end{array}\right) & :=\left(\begin{array}{cc}
X_{11} & X_{12} \\
X_{21} & X_{22}
\end{array}\right)\left(\begin{array}{cc}
1 & \bar{\lambda} \\
0 & I_{p^{\prime}}
\end{array}\right)\left(\begin{array}{cc}
1 & 0 \\
-\bar{\mu} X_{11} & I_{p^{\prime}}
\end{array}\right) \\
& =\left(\begin{array}{cc}
0 & X_{11} \bar{\lambda}+X_{12} \\
X_{21}-\left(X_{21} \bar{\lambda}+X_{22}\right) \bar{\mu} X_{11} & X_{21} \bar{\lambda}+X_{22}
\end{array}\right)
\end{aligned}
$$

We note that the last matrix in (54) defines an automorphism of $L^{\prime}$ since $R^{\prime} \bar{\mu}=0$. Using (52), the left $D$-isomorphism $\varpi$ is then defined by:

$$
\begin{aligned}
\varpi: M & \longrightarrow N \\
\pi(\lambda) & \longmapsto \pi^{\prime}\left(\lambda\left(X_{22}^{\prime}+\left(X_{21}^{\prime}-X_{22}^{\prime} \bar{\mu}\right) X_{12}^{\prime}\right)\right) .
\end{aligned}
$$

Remark 14. We note that the matrix defining (56) can be obtained from $X^{\prime}$ as the entry at position $(2,2)$ of the following matrix:

$$
\left(\begin{array}{cc}
1 & 0 \\
X_{21}^{\prime}-X_{22}^{\prime} \bar{\mu} & I_{p}
\end{array}\right)\left(\begin{array}{cc}
0 & X_{12}^{\prime} \\
X_{21}^{\prime} & X_{22}^{\prime}
\end{array}\right)=\left(\begin{array}{cc}
0 & X_{12}^{\prime} \\
X_{21}^{\prime} & X_{22}^{\prime}+\left(X_{21}^{\prime}-X_{22}^{\prime} \bar{\mu}\right) X_{12}^{\prime}
\end{array}\right) .
$$

Let us explicitly describe the algorithm realizing Stafford's cancellation theorem (see Theorem 11).

$\mathrm{RR} \mathrm{n}^{\circ} 8225$ 


\section{Algorithm 6 (CancellationTheorem).}

- Input: A very simple domain $D$ satisfying (8), $R \in D^{q \times p}$ such that the rank of the left $D$-module $M=D^{1 \times p} /\left(D^{1 \times q} R\right)$ is at least two, $R^{\prime} \in D^{q^{\prime} \times p^{\prime}}, P=\left(\begin{array}{ll}0 & R\end{array}\right) \in D^{q \times(1+p)}$, $P^{\prime}=\left(\begin{array}{ll}0 & R^{\prime}\end{array}\right) \in D^{q^{\prime} \times\left(1+p^{\prime}\right)}$, and $X \in D^{(1+p) \times\left(1+p^{\prime}\right)}$ defining the left $D$-isomorphism (53).

- Output: $Y \in D^{p \times p^{\prime}}$ defining the left $D$-isomorphism (56).

1. Select the first row $\left(X_{11} \quad X_{12}\right)$ of $X$, where $X_{11} \in D$ and $X_{12} \in D^{1 \times p^{\prime}}$.

2. Compute $Q^{\prime} \in D^{p^{\prime} \times m^{\prime}}$ such that $\operatorname{ker}_{D}\left(R^{\prime}\right.$. $)=Q^{\prime} D^{m^{\prime}}$.

3. Compute a right inverse $\left(\begin{array}{rr}\widetilde{e} & \widetilde{\mu}^{T}\end{array}\right)^{T} \in D^{1+m^{\prime}}$ of $\left(X_{11} \quad X_{12} Q^{\prime}\right)$.

4. Apply Algorithm 5 to $\left(d^{\star}, m^{\star}\right)=\left(X_{11}, \pi^{\prime}\left(X_{12}\right)\right) \in \mathrm{U}(D \oplus N), e=\widetilde{e}, \mu=Q^{\prime} \widetilde{\mu}$, where $N=D^{1 \times p^{\prime}} /\left(D^{1 \times q^{\prime}} R^{\prime}\right)$ and $\pi^{\prime}: D^{1 \times p^{\prime}} \longrightarrow N$ is the canonical projection onto $N$, to get $\bar{\lambda} \in D^{1 \times p^{\prime}}$ and $\bar{\mu} \in \operatorname{ker}_{D}\left(R^{\prime}\right.$.) such that:

$$
\left(X_{12}+X_{11} \bar{\lambda}\right) \bar{\mu}=1 .
$$

5. Compute the following matrices:

$$
X_{12}^{\prime}:=X_{11} \bar{\lambda}+X_{12}, \quad X_{21}^{\prime}:=X_{21}-\left(X_{21} \bar{\lambda}+X_{22}\right) \bar{\mu} X_{11}, \quad X_{22}^{\prime}:=X_{21} \bar{\lambda}+X_{22} .
$$

6. Return $Y:=X_{22}^{\prime}+\left(X_{21}^{\prime}-X_{22}^{\prime} \bar{\mu}\right) X_{12}^{\prime}$.

Algorithm 6 will be illustrated in the next section.

\subsection{Applications}

\subsubsection{Computation of bases}

Let $D$ be a very simple domain satisfying (8) and $M$ a finitely generated stably free left $D$ module. By definition (see Definition 1), there exist $r, s \in \mathbb{Z}_{\geq 0}$ such that $M \oplus D^{1 \times s} \cong D^{1 \times r}$. Since $D$ is a noetherian domain, we have $0 \leq s \leq r$ (see, e.g., [24]). If $\operatorname{rank}_{D}(M)=r-s \geq 2$, then applying Stafford's cancellation theorem (see Theorem 11), we obtain $M \cong D^{1 \times(r-s)}$, i.e., $M$ is a free left $D$-module of rank $r-s \geq 2$, which gives a proof of Theorem 6 .

This approach to compute bases of free left $A_{n}(k)$-modules of rank at least two was pursued in [20]. Below, we give a proof which is different from the one in [20]. Indeed, the approach of [20] is based on versions of Lemma 6 and Theorem 11 for a left/right $D$ submodule $M$ of a finitely generated free left/right $D$-module, and not for a general finitely generated (i.e., presented) left/right $D$-module $M$ as it is done in this paper. In other words, [20] considers $\operatorname{ker}_{D}(R$. $) \subseteq D^{p}$ whereas we consider the finitely presented left $D$-module $\operatorname{coker}_{D}(. R)=D^{1 \times p} /\left(D^{1 \times q} R\right)$. 
Given a presentation matrix of a finitely generated stably free left $D$-module $L$, in [36], it is shown how to compute a matrix $R \in D^{q \times p}$ which admits a right inverse $S \in D^{p \times q}$, i.e., $R S=I_{q}$, and is such that the left $D$-module $M:=D^{1 \times p} /\left(D^{1 \times q} R\right)$ is isomorphic to $L$. Then, the following short exact sequence

$$
0 \longrightarrow D^{1 \times q} \stackrel{. R}{\longrightarrow} D^{1 \times p} \stackrel{\pi}{\longrightarrow} M \longrightarrow 0
$$

splits, namely, $D^{1 \times q} \oplus M \cong D^{1 \times p}$ (see, e.g., [38]), and this left $D$-isomorphism is defined by:

$$
\begin{aligned}
& g: D^{1 \times q} \oplus M \quad \longrightarrow \quad D^{1 \times p} \\
& (\theta, \pi(\lambda)) \longmapsto\left(\begin{array}{ll}
\theta & \lambda
\end{array}\right)\left(\begin{array}{c}
R \\
I_{p}-S R
\end{array}\right), \begin{aligned}
g^{-1}: D^{1 \times p} & \longrightarrow D^{1 \times q} \oplus M \\
\lambda & \longmapsto
\end{aligned}
\end{aligned}
$$

For more details, see [35]. Let $P=\left(\begin{array}{ll}0 & R\end{array}\right) \in D^{q \times(q+p)}, P^{\prime}=0, X=\left(\begin{array}{ll}R^{T} & \left(I_{p}-S R\right)^{T}\end{array}\right)^{T}$ the above matrix defining the left $D$-isomorphism $g, L=D^{1 \times(q+p)} /\left(D^{1 \times q} P\right), L^{\prime}=D^{1 \times p}$, $\tau: D^{1 \times(q+p)} \longrightarrow L$ and $\tau^{\prime}=\operatorname{id}_{L^{\prime}}$. Using the left $D$-isomorphism $L \cong D^{1 \times q} \oplus M$ defined by sending $\tau\left(\left(\begin{array}{ll}\theta & \lambda\end{array}\right)\right)$ to $(\theta, \pi(\lambda))$ for all $\theta \in D^{1 \times q}$ and for all $\lambda \in D^{1 \times p}$, then we get the following left $D$-isomorphism:

$$
\begin{aligned}
f: L & \longrightarrow L^{\prime}=D^{1 \times p} \\
\tau\left(\left(\begin{array}{ll}
\theta & \lambda)
\end{array}\right)\right. & \longmapsto\left(\begin{array}{cc}
\theta & \lambda
\end{array}\right)\left(\begin{array}{c}
R \\
I_{p}-S R
\end{array}\right) .
\end{aligned}
$$

If $\operatorname{rank}_{D}(M)=p-q \geq 2$, then applying $q$ times Algorithm 6, we obtain a left $D$-isomorphism $h: M \longrightarrow D^{1 \times(p-q)}$ defined by $h(\pi(\lambda))=\lambda Q$ for a certain matrix $Q \in D^{p \times(p-q)}$ (called an injective parametrization in $[11,36])$. Moreover, the matrix $Q$ necessarily admits a left inverse $T \in D^{(p-q) \times p}$, i.e., $T Q=I_{p-q}$, and $\left\{\pi\left(T_{i \bullet}\right)\right\}_{i=1, \ldots, p-q}$ forms a basis of the free left $D$-module $M$ of rank $p-q$, where $T_{i}$ denotes the $i^{\text {th }}$ row of $T$. For more details, see [36].

Remark 15. Theorem 11 is based on solving quadratic equations of the form (10). As already noticed in [36], for the computation of bases of finitely generated free modules, we only need to find $\alpha, \beta \in D$ such that

$$
d_{1} D+d_{2} D+d_{3} D=\left(d_{1}+d_{3} \alpha\right) D+\left(d_{2}+d_{3} \beta\right) D,
$$

which is generally faster and gives smaller results than solving equations of the form (10). Indeed, $f(\tau(1 \quad 0))=R_{1} \bullet \in \mathrm{U}\left(D^{1 \times p}\right)$ (i.e., $R_{1} \bullet$ admits a right inverse, e.g., the first column of $S$ ) and Algorithm 5 aims at finding $\bar{\lambda} \in D^{1 \times(p-1)}$ such that $X_{12}+X_{11} \bar{\lambda} \in \mathrm{U}\left(D^{1 \times(p-1)}\right)$, where $R_{1} \bullet=\left(\begin{array}{ll}X_{11} & X_{12}\end{array}\right), X_{11} \in D$, and $X_{12} \in D^{1 \times(p-1)}$. If $d_{1}$ and $d_{2}$ are two non-zero entries of $X_{12}$ and $d_{3}=X_{11}$, then there exist $\alpha, \beta \in D$ such that (58) holds. Since the right ideal generated by the entries of $R_{1} \bullet$ is $D$, so is the right ideal generated by the entries of $X_{12}$ but where $d_{1}$ (resp., $d_{2}$ ) is replaced by $d_{1}+d_{3} \alpha$ (resp., $d_{2}+d_{3} \beta$ ). Then, $\bar{\lambda}$ can be chosen as the vector defined by $\alpha$ (resp., $\beta$ ) at the position corresponding to the one of $d_{1}$ (resp., $d_{2}$ ), and 0 elsewhere. Compare with [36]. 
Example 17. Let $D=A_{1}(\mathbb{Q}), R=\left(\begin{array}{lll}\partial & t & 0\end{array}\right)$, and $M=D^{1 \times 3} /(D R)$. Since $R$ admits the right inverse $S=\left(\begin{array}{lll}t & -\partial & 0\end{array}\right)^{T}, M$ is a stably free left $D$-module of rank 2, i.e., a free left $D$-module of rank 2 (see 4 of Theorem 1). Using Algorithm 6, let us compute a basis. Let $P=\left(\begin{array}{ll}0 & R\end{array}\right) \in D^{1 \times 4}, L=D^{1 \times 4} /(D P), \tau: D^{1 \times 4} \longrightarrow L$ be the canonical projection onto $L, L^{\prime}=D^{1 \times 3}$, and the left $D$-isomorphism $f: L \longrightarrow L^{\prime}$ defined by (57), i.e., which sends $\tau\left(\left(\begin{array}{ll}\theta & \lambda\end{array}\right)\right)$ to $\left(\begin{array}{ll}\theta & \lambda\end{array}\right) X$, where:

$$
X=\left(\begin{array}{c}
R \\
I_{p}-S R
\end{array}\right)=\left(\begin{array}{ccc}
\partial & t & 0 \\
-t \partial+1 & -t^{2} & 0 \\
\partial^{2} & t \partial+2 & 0 \\
0 & 0 & 1
\end{array}\right)
$$

Let us define:

$$
X_{11}=\partial, \quad X_{12}=\left(\begin{array}{ll}
t & 0
\end{array}\right), \quad X_{21}=\left(\begin{array}{c}
-t \partial+1 \\
\partial^{2} \\
0
\end{array}\right), \quad X_{22}=\left(\begin{array}{cc}
-t^{2} & 0 \\
t \partial+2 & 0 \\
0 & 1
\end{array}\right) .
$$

Using Remark 15 and $t D+0 D+\partial D=t D+(0+\partial) D$, we can take $\alpha=0$ and $\beta=1$ and get $\bar{\lambda}=\left(\begin{array}{ll}0 & 1\end{array}\right)$ which is such that $X_{12}+X_{11} \bar{\lambda}=\left(\begin{array}{ll}t & \partial\end{array}\right) \in \mathrm{U}\left(D^{1 \times 2}\right)$ since $X_{12}+X_{11} \bar{\lambda}$ admits the right inverse $\bar{\mu}=\left(\begin{array}{ll}-\partial & t\end{array}\right)^{T}$. Computing the matrix $X^{\prime}$ defined by (55), we get

$$
X_{11}^{\prime}=0, \quad X_{12}^{\prime}=\left(\begin{array}{ll}
t & \partial
\end{array}\right), \quad X_{21}^{\prime}=\left(\begin{array}{c}
-t \partial+1 \\
\partial^{2} \\
-t \partial
\end{array}\right), \quad X_{22}^{\prime}=\left(\begin{array}{cc}
-t^{2} & -t \partial+1 \\
t \partial+2 & \partial^{2} \\
0 & 1
\end{array}\right),
$$

which yields:

$$
Q=X_{22}^{\prime}+\left(X_{21}^{\prime}-X_{22}^{\prime} \bar{\mu}\right) X_{12}^{\prime}=\left(\begin{array}{cc}
-t^{2}(\partial+1) & -t \partial^{2}+(1-t) \partial+1 \\
t \partial^{2}+(t+2) \partial+2 & \partial^{2}(\partial+1) \\
-t(t(\partial+1)+1) & -t \partial(\partial+1)+1
\end{array}\right) .
$$

We can check that $\operatorname{ker}_{D}(. Q)=D R$, and $Q$ admits the following left inverse:

$$
T=\left(\begin{array}{ccc}
0 & t \partial+t+2 & \partial(\partial+1) \\
t & -t^{2} & -t(\partial+1)+1
\end{array}\right)
$$

Therefore, we have $M \cong D^{1 \times 3} Q=D^{1 \times 2}$ and $\left\{\pi\left(T_{i \bullet}\right)\right\}_{i=1,2}$ is a basis of $M$. 


\subsubsection{Rank reduction compatible with isomorphism}

Let us consider a finitely generated left $D$-module $M$ with $\operatorname{rank}_{D}(M) \geq 2$. Using Theorem 7 , there exist $r \in \mathbb{Z}_{\geq 0}$ and a finitely generated left $D$-module $M^{\prime}$ with $\operatorname{rank}_{D}\left(M^{\prime}\right) \leq 1$ such that:

$$
M \cong D^{1 \times r} \oplus M^{\prime}
$$

Let us also suppose that we have another direct sum decomposition $M \cong D^{1 \times s} \oplus M^{\prime \prime}$, where $\operatorname{rank}_{D}\left(M^{\prime \prime}\right) \leq 1$. Let us now study the relations between $M^{\prime}$ and $M^{\prime \prime}$. The left $D$-isomorphism $D^{1 \times r} \oplus M^{\prime} \cong D^{1 \times s} \oplus M^{\prime \prime}$ and the fact that $D$ is a noetherian domain imply:

$$
\operatorname{rank}_{D}(M)=r+\operatorname{rank}_{D}\left(M^{\prime}\right)=s+\operatorname{rank}_{D}\left(M^{\prime \prime}\right) .
$$

Then, applying Stafford's cancellation theorem (see Theorem 11), one of the following implications holds:

1. If $M^{\prime}$ and $M^{\prime \prime}$ are two torsion left $D$-modules, then $r=s \geq 2$ and:

$$
D^{1 \times(r-2)} \oplus\left(D^{1 \times 2} \oplus M^{\prime}\right) \cong D^{1 \times(r-2)} \oplus\left(D^{1 \times 2} \oplus M^{\prime \prime}\right) \quad \Rightarrow \quad D^{1 \times 2} \oplus M^{\prime} \cong D^{1 \times 2} \oplus M^{\prime \prime} .
$$

2. If $\operatorname{rank}_{D}\left(M^{\prime}\right)=\operatorname{rank}_{D}\left(M^{\prime \prime}\right)=1$, then $r=s \geq 1$ and:

$$
D^{1 \times(r-1)} \oplus\left(D \oplus M^{\prime}\right) \cong D^{1 \times(r-1)} \oplus\left(D \oplus M^{\prime \prime}\right) \quad \Rightarrow \quad D \oplus M^{\prime} \cong D \oplus M^{\prime \prime} .
$$

3. If $\operatorname{rank}_{D}\left(M^{\prime}\right)=1$ and $M^{\prime \prime}$ is a torsion left $D$-module, then $s=r+1 \geq 2$ and:

$$
D^{1 \times(r-1)} \oplus\left(D \oplus M^{\prime}\right) \cong D^{1 \times(s-2)} \oplus\left(D^{1 \times 2} \oplus M^{\prime \prime}\right) \quad \Rightarrow \quad D \oplus M^{\prime} \cong D^{1 \times 2} \oplus M^{\prime \prime} .
$$

4. If $M^{\prime}$ is a torsion left $D$-module and $\operatorname{rank}_{D}\left(M^{\prime \prime}\right)=1$, then $r=s+1 \geq 2$ and:

$$
D^{1 \times(r-2)} \oplus\left(D^{1 \times 2} \oplus M^{\prime}\right) \cong D^{1 \times(s-1)} \oplus\left(D \oplus M^{\prime \prime}\right) \quad \Rightarrow \quad D^{1 \times 2} \oplus M^{\prime} \cong D \oplus M^{\prime \prime} .
$$

\subsubsection{Auslander transposes}

Auslander transposes play a fundamental role in the study of the module structure of Auslander regular rings. For more details, see [11, 16, 34] and the references therein. Let us introduce this concept and its main properties.

Theorem 12 ([16]). Let us consider the following finite presentations of a left D-module $M$ :

$$
\begin{array}{llllll}
D^{1 \times q} & \stackrel{. R}{\longrightarrow} & D^{1 \times p} & \stackrel{\pi}{\longrightarrow} & M & \longrightarrow 0, \\
D^{1 \times q^{\prime}} & \stackrel{. R^{\prime}}{\longrightarrow} & D^{1 \times p^{\prime}} & \stackrel{\pi^{\prime}}{\longrightarrow} & M & \longrightarrow 0 .
\end{array}
$$

$\mathrm{RR} \mathrm{n}^{\circ} 8225$ 
Let us consider the following two finitely presented right D-modules:

$$
N:=D^{q} /\left(R D^{p}\right), \quad N^{\prime}:=D^{q^{\prime}} /\left(R^{\prime} D^{p^{\prime}}\right) .
$$

Then, we have

$$
D^{q^{\prime}+p} \oplus N \cong D^{q+p^{\prime}} \oplus N^{\prime},
$$

as right D-modules. Hence, $N$ and $N^{\prime}$ are projectively equivalent, which shows that $M$ defines a unique projective equivalence class. Any representative of this projective equivalence class is called an Auslander transpose of $M$. In particular, we have:

$$
\operatorname{rank}_{D}(N)+q^{\prime}+p=\operatorname{rank}_{D}\left(N^{\prime}\right)+q+p^{\prime} .
$$

We refer to [16] for the explicit construction of a right $D$-isomorphism as in (59).

The Auslander transpose of a finitely presented left $D$-module is a finitely presented right $D$-module. If $D=A\left\langle\partial_{1}, \ldots, \partial_{n}\right\rangle$, then using the involution $\theta$ of $D$ defined by (9), the right $D$-module structure on an Auslander transpose can be turned into a left $D$-module structure. More precisely, if we introduce the so-called adjoint matrix $\widetilde{R}:=\left(\theta\left(R_{i j}\right)\right)^{T} \in D^{p \times q}$ of $R \in D^{q \times p}$, then the left $D$-module $\widetilde{N}:=D^{1 \times q} /\left(D^{1 \times p} \widetilde{R}\right)$ is called the adjoint of the left $D$-module $M=D^{1 \times p} /\left(D^{1 \times q} R\right)$. Then, (59) yields:

$$
D^{1 \times\left(q^{\prime}+p\right)} \oplus \widetilde{N} \cong D^{1 \times\left(q+p^{\prime}\right)} \oplus \widetilde{N^{\prime}} .
$$

Turning right $D$-modules into left $D$-modules by means of an involution of the ring is usually useful in practice since many computer algebra systems (e.g., Maple) can only handle left Gröbner basis techniques. In mathematical physics, adjoint modules play an important role as shown in [31] (see also the references therein and [16]).

Corollary 4. Let $D$ be a very simple domain. With the notations of Theorem 12, if $r=q^{\prime}+p$, $r^{\prime}=q+p^{\prime}$, and $s \in \mathbb{Z}_{\geq 0}$ is such that

$$
\operatorname{rank}_{D}(N)+r-s=\operatorname{rank}_{D}\left(N^{\prime}\right)+r^{\prime}-s \geq 2,
$$

(59) yields:

$$
D^{r-s} \oplus N \cong D^{r^{\prime}-s} \oplus N^{\prime}
$$

Moreover, if $D$ admits an involution, then:

$$
D^{1 \times(r-s)} \oplus \widetilde{N} \cong D^{1 \times\left(r^{\prime}-s\right)} \oplus \widetilde{N^{\prime}}
$$

Example 18. Let us consider the first Weyl algebra $D=A_{1}(\mathbb{Q}), R^{\prime}=\left(\begin{array}{ll}t^{2} & t \partial+2\end{array}\right)^{T}$,

$$
R=\left(\begin{array}{cc}
t^{2} & t \\
t \partial+2 & \partial
\end{array}\right),
$$

$\mathrm{RR} \mathrm{n}^{\circ} 8225$ 
$W=(1 \quad 0), M=D^{1 \times 2} /\left(D^{1 \times 2} R\right)$, and $M^{\prime}=D /\left(D^{1 \times 2} R^{\prime}\right)$. If $\pi: D^{1 \times 2} \longrightarrow M$ (resp., $\left.\pi^{\prime}: D \longrightarrow M^{\prime}\right)$ is the canonical projection onto $M$ (resp., $M^{\prime}$ ), then Example 11 shows that

$$
\begin{aligned}
\chi: M^{\prime}=D /\left(D^{1 \times 2} R^{\prime}\right) & \longrightarrow M=D^{1 \times 2} /\left(D^{1 \times 2} R\right) \\
\pi^{\prime}(d) & \longmapsto \pi(d W),
\end{aligned}
$$

is a left $D$-isomorphism, i.e., $M^{\prime} \cong M$. Let $N^{\prime}=D^{2} /\left(R^{\prime} D\right)$ (resp., $\left.N=D^{2} /\left(R D^{2}\right)\right)$ be the Auslander transpose of $M^{\prime}$ (resp., $M$ ). Then, Theorem 12 yields $D^{4} \oplus N \cong D^{3} \oplus N^{\prime}$. Using the involution $\theta$ of $D=A_{1}(\mathbb{Q})$ defined by (9), (60) yields

$$
D^{1 \times 4} \oplus \widetilde{N} \cong D^{1 \times 3} \oplus \widetilde{N^{\prime}}
$$

where $\widetilde{N}=D^{1 \times 2} /\left(D^{1 \times 2} \widetilde{R}\right)\left(\right.$ resp., $\left.\widetilde{N^{\prime}}=D^{1 \times 2} /\left(D \widetilde{R^{\prime}}\right)\right)$ is the adjoint of $M$ (resp., $\left.M^{\prime}\right)$, where:

$$
\widetilde{R}=\left(\begin{array}{cc}
t^{2} & -t \partial+1 \\
t & -\partial
\end{array}\right), \quad \widetilde{R^{\prime}}=\left(\begin{array}{ll}
t^{2} & -t \partial+1
\end{array}\right)
$$

More precisely, using the OreMorPhISMS package ([14]), we obtain

$$
D^{1 \times 4} \oplus \widetilde{N} \cong L:=D^{1 \times 7} /\left(D^{1 \times 3} P\right), \quad D^{1 \times 3} \oplus \widetilde{N^{\prime}} \cong L^{\prime}:=D^{1 \times 7} /\left(D^{1 \times 3} P^{\prime}\right),
$$

where

$$
P=\left(\begin{array}{ccccccc}
0 & 0 & 0 & 0 & 1 & 0 & 0 \\
0 & 0 & 0 & 0 & 0 & t^{2} & -t \partial+1 \\
0 & 0 & 0 & 0 & 0 & t & -\partial
\end{array}\right), \quad P^{\prime}=\left(\begin{array}{ccccccc}
0 & 0 & 0 & t^{2} & -t \partial+1 & 0 & 0 \\
0 & 0 & 0 & 0 & 0 & 1 & 0 \\
0 & 0 & 0 & 0 & 0 & 0 & 1
\end{array}\right)
$$

and the following left $D$-isomorphism

$$
\begin{aligned}
f: L=D^{1 \times 7} /\left(D^{1 \times 3} P\right) & \longrightarrow L^{\prime}=D^{1 \times 7} /\left(D^{1 \times 3} P^{\prime}\right) \\
\tau(\zeta) & \longmapsto \tau^{\prime}(\zeta X),
\end{aligned}
$$

where

$$
X=\left(\begin{array}{ccccccc}
0 & -1 & 0 & 1 & 0 & 0 & 0 \\
0 & 0 & -1 & 0 & 1 & 0 & 0 \\
1 & -t^{2} & t \partial-1 & 0 & 0 & 1 & 0 \\
0 & -t & \partial & 0 & 0 & 0 & 1 \\
0 & 0 & 0 & t^{2} & -t \partial+1 & -1 & 0 \\
0 & 0 & 0 & t \partial+2 & -\partial^{2} & 0 & -\partial \\
0 & 0 & 0 & t^{2} & -t \partial+1 & 0 & -t
\end{array}\right),
$$

and $\tau: D^{1 \times 7} \longrightarrow L$ (resp., $\tau^{\prime}: D^{1 \times 7} \longrightarrow L^{\prime}$ ) is the canonical projection onto $L$ (resp., $L^{\prime}$ ). For more details, see [16].

$\mathrm{RR} \mathrm{n}^{\circ} 8225$ 
We can check that $\operatorname{rank}_{D}(\widetilde{N})=0$ and $\operatorname{rank}_{D}\left(\widetilde{N^{\prime}}\right)=1$. Corollary 4 then implies:

$$
D^{1 \times 2} \oplus \widetilde{N} \cong D \oplus \widetilde{N^{\prime}}
$$

Using Algorithm 6, let us explicitly describe the above isomorphism. Let $L_{2}=D^{1 \times 6} /\left(D^{1 \times 3} P_{2}\right)$, and $L_{2}^{\prime}=D^{1 \times 6} /\left(D^{1 \times 3} P_{2}^{\prime}\right)$, where

$$
\begin{aligned}
& P_{2}=\left(\begin{array}{cccccc}
0 & 0 & 0 & 1 & 0 & 0 \\
0 & 0 & 0 & 0 & t^{2} & -t \partial+1 \\
0 & 0 & 0 & 0 & t & -\partial
\end{array}\right), \quad P_{2}^{\prime}=\left(\begin{array}{cccccc}
0 & 0 & t^{2} & -t \partial+1 & 0 & 0 \\
0 & 0 & 0 & 0 & 1 & 0 \\
0 & 0 & 0 & 0 & 0 & 1
\end{array}\right) \text {, } \\
& X_{11}=0, X_{12}=\left(\begin{array}{llllll}
-1 & 0 & 1 & 0 & 0 & 0
\end{array}\right), X_{21}=\left(\begin{array}{llllll}
0 & 1 & 0 & 0 & 0 & 0
\end{array}\right)^{T} \text {, and: } \\
& X_{22}=\left(\begin{array}{cccccc}
0 & -1 & 0 & 1 & 0 & 0 \\
-t^{2} & t \partial-1 & 0 & 0 & 1 & 0 \\
-t & \partial & 0 & 0 & 0 & 1 \\
0 & 0 & t^{2} & -t \partial+1 & -1 & 0 \\
0 & 0 & t \partial+2 & -\partial^{2} & 0 & -\partial \\
0 & 0 & t^{2} & -t \partial+1 & 0 & -t
\end{array}\right), \quad Q_{1}^{\prime}=\left(\begin{array}{ccc}
1 & 0 & 0 \\
0 & 1 & 0 \\
0 & 0 & -\partial \\
0 & 0 & -t \\
0 & 0 & 0 \\
0 & 0 & 0
\end{array}\right) .
\end{aligned}
$$

We can check that $\operatorname{ker}_{D}\left(P_{2}^{\prime}.\right)=Q_{1}^{\prime} D^{3}$. Since $X_{11}=0, X_{12} Q_{1}^{\prime}$ necessarily admits a right inverse, e.g., $\nu_{1}=\left(\begin{array}{lll}-1 & 0 & 0\end{array}\right)$, which yields $\bar{\mu}_{1}=Q_{1}^{\prime} \nu_{1}=\left(\begin{array}{llllll}-1 & 0 & 0 & 0 & 0 & 0\end{array}\right)^{T}$. Thus, $X_{12} \bar{\mu}_{1}=1$ and $\bar{\mu}_{1} \in \operatorname{ker}_{D}\left(P_{2}^{\prime}\right.$.). Now, (54) with $X_{11}=0$ and $\bar{\lambda}=0$ implies that $X^{\prime}=X$, and thus (56) then gives

$$
Y:=X_{22}+\left(X_{21}-X_{22} \bar{\mu}\right) X_{12}=\left(\begin{array}{cccccc}
0 & -1 & 0 & 1 & 0 & 0 \\
-1 & t \partial-1 & -t^{2}+1 & 0 & 1 & 0 \\
0 & \partial & -t & 0 & 0 & 1 \\
0 & 0 & t^{2} & -t \partial+1 & -1 & 0 \\
0 & 0 & t \partial+2 & -\partial^{2} & 0 & -\partial \\
0 & 0 & t^{2} & -t \partial+1 & 0 & -t
\end{array}\right),
$$

which yields the following left $D$-isomorphism

$$
\begin{aligned}
f_{2}: L_{2}=D^{1 \times 6} /\left(D^{1 \times 3} P_{2}\right) & \longrightarrow L_{2}^{\prime}=D^{1 \times 6} /\left(D^{1 \times 3} P_{2}^{\prime}\right) \\
\tau_{2}(\xi) & \longmapsto \tau_{2}^{\prime}(\xi Y),
\end{aligned}
$$

where $L_{2} \cong D^{1 \times 3} \oplus \widetilde{N}$ and $L_{2}^{\prime} \cong D^{1 \times 2} \oplus \widetilde{N^{\prime}}$. Finally, let us apply Algorithm 6 to $f_{2}$. Let

$$
P_{3}=\left(\begin{array}{ccccc}
0 & 0 & 1 & 0 & 0 \\
0 & 0 & 0 & t^{2} & -t \partial+1 \\
0 & 0 & 0 & t & -\partial
\end{array}\right), \quad P_{3}^{\prime}=\left(\begin{array}{ccccc}
0 & t^{2} & -t \partial+1 & 0 & 0 \\
0 & 0 & 0 & 1 & 0 \\
0 & 0 & 0 & 0 & 1
\end{array}\right)
$$

$\mathrm{RR} \mathrm{n}^{\circ} 8225$ 


$$
\begin{aligned}
& L_{3}=D^{1 \times 5} /\left(D^{1 \times 3} P_{3}\right), L_{3}^{\prime}=D^{1 \times 5} /\left(D^{1 \times 3} P_{3}^{\prime}\right), Y_{11}=0, Y_{12}=\left(\begin{array}{lllll}
-1 & 0 & 1 & 0 & 0
\end{array}\right) \text {, and: } \\
& Y_{21}=\left(\begin{array}{c}
-1 \\
0 \\
0 \\
0 \\
0
\end{array}\right), Y_{22}=\left(\begin{array}{ccccc}
t \partial-1 & -t^{2}+1 & 0 & 1 & 0 \\
\partial & -t & 0 & 0 & 1 \\
0 & t^{2} & -t \partial+1 & -1 & 0 \\
0 & t \partial+2 & -\partial^{2} & 0 & -\partial \\
0 & t^{2} & -t \partial+1 & 0 & -t
\end{array}\right), Q_{2}^{\prime}=\left(\begin{array}{cc}
1 & 0 \\
0 & -\partial \\
0 & -t \\
0 & 0 \\
0 & 0
\end{array}\right) .
\end{aligned}
$$

We can check that $\operatorname{ker}_{D}\left(P_{3}^{\prime}.\right)=Q_{2}^{\prime} D^{2}$. Since $Y_{11}=0, Y_{12} Q_{2}^{\prime}$ necessarily admits a right inverse, e.g., $\nu_{2}=\left(\begin{array}{ll}-1 & 0\end{array}\right)^{T}$, which yields $\bar{\mu}_{2}=Q_{2}^{\prime} \nu_{2}=\left(\begin{array}{lllll}-1 & 0 & 0 & 0 & 0\end{array}\right)^{T}$. Thus, we have $Y_{12} \bar{\mu}_{2}=1$ and $\bar{\mu}_{2} \in \operatorname{ker}_{D}\left(P_{3}^{\prime}\right.$.). Using (55) with $\bar{\lambda}=0$ and $\bar{\mu}=\bar{\mu}_{2}$, we get

$$
Z:=Y_{22}+\left(Y_{21}-Y_{22} \bar{\mu}\right) X_{12}=\left(\begin{array}{ccccc}
1 & -t^{2}+1 & t \partial-2 & 1 & 0 \\
0 & -t & \partial & 0 & 1 \\
0 & t^{2} & -t \partial+1 & -1 & 0 \\
0 & t \partial+2 & -\partial^{2} & 0 & -\partial \\
0 & t^{2} & -t \partial+1 & 0 & -t
\end{array}\right)
$$

which yields the following left $D$-isomorphism

$$
\begin{aligned}
f_{3}: L_{3}=D^{1 \times 5} /\left(D^{1 \times 3} P_{3}\right) & \longrightarrow L_{3}^{\prime}=D^{1 \times 5} /\left(D^{1 \times 3} P_{3}^{\prime}\right) \\
\tau_{3}(\nu) & \longmapsto \tau_{3}^{\prime}(\nu Z),
\end{aligned}
$$

and finally proves that $D^{1 \times 2} \oplus \widetilde{N} \cong L_{3} \cong L_{3}^{\prime} \cong D \oplus \widetilde{N^{\prime}}$.

\section{Conclusion}

The constructive study of Stafford's theorems ([42]) on the module structure of the Weyl algebras $A_{n}(k)$ over a field $k$ of characteristic zero was initiated in [21,25] and continued in [20, 36]. In the present paper, we have studied the rest of Stafford's theorems from a constructive viewpoint. The corresponding algorithms have been implemented in the STAFForD package ([36]) and the main results of the paper were illustrated with explicit and classical linear systems of PD equations. Finally, we have also explained that Stafford's theorems could be extended to the case of rings of PD operators with coefficients in different differential rings or fields, or more generally, to any very simple domain.

We hope that the results of this paper and those of $[20,36]$ will motivate the symbolic computation community to pay more attention to constructive versions of Stafford's main theorem (see (6)). To be able to efficiently compute two "simple generators" of ideals of $A_{n}(k)$ is the main issue for studying the module structure of $A_{n}(k)$ (e.g., by means of the

$\mathrm{RR} \mathrm{n}^{\circ} 8225$ 
algorithms in the present paper) and using it in the study of linear PD systems within algebraic analysis or $D$-module theory.

The feasibility of an implementation of the extensions of Stafford's theorems (see Theorems 4 and 5) in a computer algebra system also needs to be studied in the future. For analytic linear OD systems, an extension of the STAFFord package called STAFFordANALYTIC is under development.

Stafford's theorems ([42]) have been generalized in another important direction in [18] where it was shown that the module structure of the ring $\mathcal{D}(X)$ of $\mathrm{PD}$ operators on a smooth irreducible affine variety $X$ over a field $k$ of characteristic zero is similar to the one of the Weyl algebra $A_{n}(k)=\mathcal{D}\left(\mathbb{A}^{n}(k)\right)$, where $\mathbb{A}^{n}(k)$ denotes affine space of dimension $n$ over $k$. The only differences are that one-sided ideals can now be generated by three elements instead of two and the condition $\operatorname{rank}_{A_{n}(k)}(M) \geq 2$ in the theorems of [42] and of this paper has to be replaced by $\operatorname{rank}_{\mathcal{D}(X)}(M) \geq 3$. These results can also be extended to certain singular affine algebraic varieties (see [18]). It was also shown in [8] that statements similar to the ones for $\mathcal{D}(X)$ hold for $A_{n}(K)$, where $K$ is a division ring of characteristic zero (e.g., the quaternions $\mathbb{H}$ or the division ring of fractions of $A_{m}(k)$ (see Section 2)). Based on the constructive versions of Stafford's theorems developed in this paper, we are currently studying these extensions.

Finally, the properties of (the category of) finitely generated modules over a very simple domain should be studied in detail (as shown in [18], it has good functorial properties such as respecting localizations with respect to Ore sets).

\section{Appendix: New commands of the STAFFORD package}

We demonstrate the new commands of the STAFFORD package dedicated to Stafford's theorems ([42]) and their applications to mathematical systems theory.

The Stafford package uses the OreModules package ([12]). To handle linear algebra operations (resp., homomorphisms), we use the Maple package linalg (resp., the OreMorPHISMS package $([14]))$.

$>$ with(OreModules):

$>$ with(OreMorphisms):

$>$ with(Stafford):

$>$ with(linalg):

Since the symbol $D$ is protected in Maple, in what follows, we shall use $A$ instead of $D$ as a name for (the data representing) an Ore algebra.

$\mathrm{RR} \mathrm{n}^{\circ} 8225$ 


\subsection{Strong generation, unimodular elements, and Serre's splitting-off the- orem}

Example 19. Let us consider the second Weyl algebra $A=A_{2}(\mathbb{Q})$ :

$$
>A:=\text { DefineOreAlgebra }(\operatorname{diff}=[\mathrm{d} 1, \mathrm{x} 1] \text {, } \operatorname{diff}=[\mathrm{d} 2, \mathrm{x} 2] \text {, polynom }=[\mathrm{x} 1, \mathrm{x} 2]) \text { : }
$$

More precisely, the procedures of the OreModules, Stafford, and OreMorphisms packages perform multiplication of polynomials in $x 1, x 2, d 1$, d2 subject to the following commutation rules:

$$
\forall i, j=1,2, \quad d i x j=x j d i+\delta_{i j}, \quad d i d j=d j d i, \quad \text { xi } x j=x j x i .
$$

Since Maple's data structure for polynomials is used here and this data structure does not retain the order of symbols in a product in general, the following results are to be understood with the convention that a monomial in $x i$ and $d j$ represents the element of the Weyl algebra that is defined by writing all $x i$ to the left of all the $d j$. For instance, even when a procedure returns the polynomial $d 1 x 1$, the element $x 1 d 1 \in A$ is referred to here (and not $x 1 d 1+1$ ).

We demonstrate the procedure TwoStrongGenerators. Given $a, b, c \in A$ and $d, e \in A \backslash\{0\}$, this procedure computes two elements $u, v \in A$ such that

$$
A a+A b+A c=A(a+d u c)+A(b+e v c)
$$

(see (7) in Lemma 4, where $d_{1}=d$ and $d_{2}=e$ ). We apply TwoStrongGenerators to the following elements $a, b, c$, and $d:=x 2, e:=x 1$.

$>\mathrm{a}:=\mathrm{d} 1 ; \mathrm{b}:=\mathrm{d} 2 ; \mathrm{c}:=\mathrm{x} 1$;

$$
\begin{aligned}
& a:=d 1 \\
& b:=d 2 \\
& c:=x 1
\end{aligned}
$$

Using the above notations, the result of the procedure TwoStrongGenerators is a list of the form $[a+d u c, b+e v c,[u, v]]$.

$$
\begin{array}{r}
>\mathrm{G}:=\text { TwoStrongGenerators }(\mathrm{a}, \mathrm{b}, \mathrm{c}, \mathrm{x} 2, \mathrm{x} 1, \mathrm{~A}) ; \\
G:=\left[d 1, d 2+x 1^{2},[0,1]\right]
\end{array}
$$

Let us check the result. Let us consider the following two matrices:

$>\mathrm{R}:=\operatorname{evalm}([[\mathrm{a}],[\mathrm{b}],[\mathrm{c}]])$;

$$
R:=\left[\begin{array}{l}
d 1 \\
d 2 \\
x 1
\end{array}\right]
$$

$\mathrm{RR} \mathrm{n}^{\circ} 8225$ 
$>\mathrm{S}:=\operatorname{evalm}([[\mathrm{G}[1]],[\mathrm{G}[2]]])$

$$
S:=\left[\begin{array}{c}
d 1 \\
d 2+x 1^{2}
\end{array}\right]
$$

The next command computes (if it exists) a matrix $F_{1} \in A^{2 \times 3}$ satisfying $S=F_{1} R$.

$>F 1:=$ Factorize $(\mathrm{S}, \mathrm{R}, \mathrm{A})$;

$$
\left[\begin{array}{ccc}
1 & 0 & 0 \\
0 & 1 & x 1
\end{array}\right]
$$

Similarly, we can check whether or not there exists a matrix $F_{2} \in A^{3 \times 2}$ such that $R=F_{2} S$.

$>$ F2 := Factorize $(\mathrm{R}, \mathrm{S}, \mathrm{A})$;

$$
\left[\begin{array}{cc}
1 & 0 \\
1 / 2 d 2 x 1+1 / 2 x 1^{3} & 1-1 / 2 d 1 x 1 \\
-1 / 2 x 1^{2}-1 / 2 d 2 & 1 / 2 d 1
\end{array}\right]
$$

Hence, $a, b, c$ can be expressed as left $A$-linear combinations of $d 1$ and $d 2+x 1^{2}$, and vice versa. In other words, we have $A a+A b+A c=A d 1+A\left(d 2+x 1^{2}\right)$.

We continue with another example.

$$
\begin{array}{rl}
>\mathrm{a}:=\mathrm{d} 1 \wedge 2 ; \mathrm{b}:=\mathrm{d} 2 \wedge 2 ; \mathrm{c}:=\mathrm{d} 1 & * \mathrm{~d} 2 ; \\
a & :=d 1^{2} \\
b & :=d 2^{2} \\
c & :=d 1 d 2
\end{array}
$$

Now, for $d=d_{1}$ and $e=d_{2}$, using the procedure TwoStrongGenerators, we compute $u$, $v \in A$ such that $A a+A b+A c=A(a+d u c)+A(b+e v c)$.

$$
\begin{gathered}
>\mathrm{G}:=\text { TwoStrongGenerators }(\mathrm{a}, \mathrm{b}, \mathrm{c}, \mathrm{d} 1, \mathrm{~d} 2, \mathrm{~A}) ; \\
G:=\left[d 1^{2}, d 2^{2}+d 2^{2}\left(x 1^{3}+x 2 x 1^{3}+x 2 x 1^{5}\right) d 1+\left(x 1^{3}+x 1^{5}\right) d 1 d 2,\right. \\
\left.\left[0, x 1^{3}+\left(x 1^{3}+x 1^{5}\right) x 2\right]\right]
\end{gathered}
$$

Let us check the result. Let us consider the following two matrices:

$>\mathrm{R}:=\operatorname{evalm}([[\mathrm{a}],[\mathrm{b}],[\mathrm{c}]]) ;$

$$
R:=\left[\begin{array}{c}
d 1^{2} \\
d 2^{2} \\
d 1 d 2
\end{array}\right]
$$

$\mathrm{RR} \mathrm{n}^{\circ} 8225$ 


$$
\begin{aligned}
>\mathrm{S}: & :=\operatorname{evalm}([\mathrm{G}[1]],[\mathrm{G}[2]]]) ; \\
& S:=\left[\begin{array}{c}
d 1^{2} \\
d 2^{2}+d 2^{2}\left(x 1^{3}+x 2 x 1^{3}+x 2 x 1^{5}\right) d 1+\left(x 1^{3}+x 1^{5}\right) d 1 d 2
\end{array}\right]
\end{aligned}
$$

The procedure Factorize, applied to $S$ and $R$, computes (if it exists) a matrix $E \in A^{2 \times 3}$ satisfying $S=E R$.

$>E:=$ Factorize $(S, R, A)$;

$$
\left[\begin{array}{ccc}
1 & 0 & 0 \\
0 & 1 & x 1^{3}+d 2 x 1^{3}+x 2 d 2 x 1^{3}+x 1^{5}+x 2 d 2 x 1^{5}
\end{array}\right]
$$

We exchange the roles of $S$ and $R$ to find a matrix $F \in A^{3 \times 2}$ such that $R=F S$.

$$
>F:=\text { Factorize }(\mathrm{R}, \mathrm{S}, \mathrm{A}):
$$

In fact, we only show one entry of $F$ here to demonstrate that the result of Factorize is not equal to FAIL (which would indicate that no matrix $F$ with the above property exists).

$>\mathrm{F}[1,1]$;

Hence, we have:

$$
\begin{gathered}
A d 1^{2}+A d 2^{2}+A d 1 d 2 \\
=A d 1^{2}+A\left(d 2^{2}+d 2^{2}\left(x 1^{3}+x 2 x 1^{3}+x 2 x 1^{5}\right) d 1+\left(x 1^{3}+x 1^{5}\right) d 1 d 2\right) .
\end{gathered}
$$

The next example deals with the third Weyl algebra $A=A_{3}(\mathbb{Q})$ :

$$
\begin{aligned}
& >A:=\text { DefineOreAlgebra }(\operatorname{diff}=[\mathrm{d} 1, \mathrm{x} 1], \operatorname{diff}=[\mathrm{d} 2, \mathrm{x} 2], \operatorname{diff}=[\mathrm{d} 3, \mathrm{x} 3], \\
& >\text { polynom }=[\mathrm{x} 1, \mathrm{x} 2, \mathrm{x} 3]):
\end{aligned}
$$

We use the procedure TwoStrongGenerators to compute two elements $u, v \in A$ such that

$$
A a+A b+A c=A(a+d u c)+A(b+e v c),
$$

where

$$
\begin{aligned}
>\mathrm{a}:=\mathrm{d} 1 ; \mathrm{b}:=\mathrm{d} 2 ; \mathrm{c}:=\mathrm{d} 3 ; & \\
a & :=d 1 \\
b & :=d 2 \\
c & :=d 3
\end{aligned}
$$

and $d=e=d_{1}$. 


$$
\begin{gathered}
>\mathrm{G}:=\text { TwoStrongGenerators }(\mathrm{a}, \mathrm{b}, \mathrm{c}, \mathrm{d} 1, \mathrm{~d} 1, \mathrm{~A}) \\
G:=\left[d 1, d 2+d 1\left(x 1^{3}+x 3 x 1^{3}+x 3 x 1^{5}\right) d 3+\left(3 x 1^{2}+3 x 1^{2} x 3+5 x 3 x 1^{4}\right) d 3,\right. \\
\left.\left[0, x 1^{3}+\left(x 1^{3}+x 1^{5}\right) x 3\right]\right]
\end{gathered}
$$

Let us check the result. Let us consider the following two matrices:

$>\mathrm{R}:=\operatorname{evalm}([[\mathrm{a}],[\mathrm{b}],[\mathrm{c}]])$;

$$
R:=\left[\begin{array}{l}
d 1 \\
d 2 \\
d 3
\end{array}\right]
$$

$>\mathrm{S}:=\operatorname{evalm}([[\mathrm{G}[1]],[\mathrm{G}[2]]])$;

$$
S:=\left[\begin{array}{c}
d 1 \\
d 2+d 1\left(x 1^{3}+x 3 x 1^{3}+x 3 x 1^{5}\right) d 3+\left(3 x 1^{2}+3 x 1^{2} x 3+5 x 3 x 1^{4}\right) d 3
\end{array}\right]
$$

We can check whether or not there exists a matrix $E \in A^{2 \times 3}$ satisfying $S=E R$ :

$>E:=$ Factorize $(\mathrm{S}, \mathrm{R}, \mathrm{A})$;

$$
\left[\begin{array}{ccc}
1 & 0 & 0 \\
0 & 1 & x 1^{5} x 3 d 1+x 1^{3} x 3 d 1+5 x 3 x 1^{4}+d 1 x 1^{3}+3 x 1^{2} x 3+3 x 1^{2}
\end{array}\right]
$$

Similarly, we can compute a matrix $F \in A^{3 \times 2}$ such that $R=F S$ :

$>F:=$ Factorize $(\mathrm{R}, \mathrm{S}, \mathrm{A}):$

We print only one entry of the result to demonstrate that Factorize computed a matrix $F$ as above.

$>\mathrm{F}[1,1]$;

We conclude that:

$$
\begin{gathered}
A d 1+A d 2+A d 3 \\
=A d 1+A\left(d 2+\left(x 1^{3}+x 3 x 1^{3}+x 3 x 1^{5}\right) d 1 d 3+\left(3 x 1^{2}+3 x 1^{2} x 3+5 x 3 x 1^{4}\right) d 3\right) .
\end{gathered}
$$

Example 20. Let us consider the third Weyl algebra $A=A_{3}(\mathbb{Q})$

$>A:=$ DefineOreAlgebra(diff $=[d[1], x[1]], \operatorname{diff}=[d[2], x[2]], \operatorname{diff}=[d[3], x[3]]$, $>$ polynom $=[\mathrm{x}[1], \mathrm{x}[2], \mathrm{x}[3]])$

and the left $A$-module $M=A^{1 \times 3} /\left(A^{1 \times 3} R\right)$ finitely presented by the following matrix $R$ : 
$>\quad \mathrm{R}:=\operatorname{evalm}([[\mathrm{x}[2] * \mathrm{~d}[1] / 2, \mathrm{x}[2] * \mathrm{~d}[2]+1, \mathrm{x}[2] * \mathrm{~d}[3]+\mathrm{d}[1] / 2]$,
$>\quad[-\mathrm{x}[2] * \mathrm{~d}[2] / 2-3 / 2,0, \mathrm{~d}[2] / 2]$,
$>\quad[-\mathrm{d}[1]-\mathrm{x}[2] * \mathrm{~d}[3] / 2,-\mathrm{d}[2],-\mathrm{d}[3] / 2]]) ;$
$R:=\left[\begin{array}{ccc}1 / 2 x_{2} d_{1} & x_{2} d_{2}+1 & x_{2} d_{3}+1 / 2 d_{1} \\ -1 / 2 x_{2} d_{2}-3 / 2 & 0 & 1 / 2 d_{2} \\ -d_{1}-1 / 2 x_{2} d_{3} & -d_{2} & -1 / 2 d_{3}\end{array}\right]$

The left $A$-module $M$ corresponds to a system defining the infinitesimal transformations of the Lie pseudogroup formed by the contact transformations. See Example 2.

Let us compute the rank of $M$ :

$>\operatorname{OreRank}(\mathrm{R}, \mathrm{A})$;

Thus, we get $\operatorname{rank}_{A}(M)=1$. Let us now study $\operatorname{hom}_{A}(M, A)$. We first compute $\operatorname{ker}_{A}(R$.).

$>\mathrm{Q}:=$ Involution(SyzygyModule(Involution (R,A), A), A);

$$
Q:=\left[\begin{array}{c}
-d_{2} \\
x_{2} d_{3}+d_{1} \\
-2-x_{2} d_{2}
\end{array}\right]
$$

We obtain $\operatorname{ker}_{A}(R)=.Q A^{3}$. In particular, let us check that $R Q=0$ :

$>\operatorname{Mult}(\mathrm{R}, \mathrm{Q}, \mathrm{A})$;

$$
\left[\begin{array}{l}
0 \\
0 \\
0
\end{array}\right]
$$

Hence, we get $\operatorname{hom}_{A}(M, A) \cong \operatorname{ker}_{A}(R)=.Q A$. A form of $M$ is then defined by means of a right multiple $Q \xi$ of $Q$, where $\xi \in A$.

A unimodular element $m^{\star}=\pi\left(\lambda^{\star}\right)$ of $M$, where $\lambda^{\star} \in A^{1 \times 3}$ and $\pi: A^{1 \times 3} \longrightarrow M$ is the canonical projection onto $M$, satisfies $\lambda^{\star}\left(Q \xi^{\star}\right)=1$ for a certain $\xi^{\star} \in A$. Since $Q$ admits a left inverse

$$
\begin{aligned}
&>\mathrm{T}:=\operatorname{LeftInverse}(\mathrm{Q}, \mathrm{A}) ; \\
& \qquad T:=\left[\begin{array}{lll}
1 / 2 x_{2} & 0 & -1 / 2
\end{array}\right]
\end{aligned}
$$

i.e., $T Q=1$, if $\lambda^{\star}=T$, then $m^{\star}=\pi\left(\lambda^{\star}\right)$ is a unimodular element of $M$ and $\varphi \in \operatorname{hom}_{A}(M, A)$ defined by $\varphi(\pi(\lambda))=\lambda Q$ for all $\lambda \in A^{1 \times 3}$ satisfies $\varphi\left(m^{\star}\right)=T Q=1$.

Serre's splitting-off theorem (see Theorem 7) cannot be used since $\operatorname{rank}_{A}(M)<2$.

$\mathrm{RR} \mathrm{n}^{\circ} 8225$ 
$>$ UnimodularElement $(\mathrm{R}, \mathrm{A})$;

Error, (in Stafford/UnimodularElementInSubmodule) expecting that the rank of the

left module presented by the first matrix is at least 2.

But, using the option "checkrank"=false, we can try to detect a unimodular element of $M$ by means of a method different from the one used in Theorem 7 (which assumes that $\operatorname{rank}_{A}(M) \geq 2$ ). See the end of Section 3.1.

$>\mathrm{U}:=$ UnimodularElement $(\mathrm{R}, \mathrm{A}$, "checkrank"=false);

$$
U:=\left[\left[\begin{array}{lll}
1 / 2 x_{2} & 0 & -1 / 2
\end{array}\right],\left[\begin{array}{c}
-d_{2} \\
x_{2} d_{3}+d_{1} \\
-2-x_{2} d_{2}
\end{array}\right]\right]
$$

We find again that $m^{\star}=\pi(U[1])=\pi(T)$ is a unimodular element of $M$ and $\varphi \in \operatorname{hom}_{A}(M, A)$, defined by $\varphi(\pi(\lambda))=\lambda U[2]$ for all $\lambda \in A^{1 \times 3}$, satisfies $\varphi\left(m^{\star}\right)=1$ :

$>\operatorname{Mult}(\mathrm{U}[1], \mathrm{U}[2], \mathrm{A})$;

$$
[1]
$$

Finally, we can check that $\varphi \in \operatorname{hom}_{A}(M, A)$ since $R U[2]=0$ :

$>\operatorname{Mult}(\mathrm{R}, \mathrm{U}[2], \mathrm{A})$;

$$
\left[\begin{array}{l}
0 \\
0 \\
0
\end{array}\right]
$$

Now, let us consider a new left $A$-module $M=A^{1 \times 3} /(A R)$ finitely presented by the following matrix $R$ :

$$
\begin{aligned}
>\mathrm{R}:=\operatorname{evalm}([\mathrm{d}[1]+\mathrm{x}[2], \mathrm{d}[2], \mathrm{d}[3]+\mathrm{x}[1]]]) ; \\
R:=\left[\begin{array}{lll}
d_{1}+x_{2} & d_{2} & d_{3}+x_{1}
\end{array}\right]
\end{aligned}
$$

The rank of $M$ is clearly 2. Let us compute a unimodular element based on Serre's splitting-off theorem (see Theorem 7).

$$
\begin{aligned}
& >\mathrm{U}:=\text { UnimodularElement }(\mathrm{R}, \mathrm{A}) ; \\
& \left.U:=\left[\begin{array}{lll}
0 & 1 & 0
\end{array}\right],\left[\begin{array}{c}
-\left(d_{3}+x_{1}+d_{2}\right)\left(d_{3}+x_{1}\right) \\
1 \\
2+d_{3} d_{1}+d_{3} x_{2}+x_{1} d_{1}+x_{1} x_{2}+d_{1} d_{2}+x_{2} d_{2}
\end{array}\right]\right]
\end{aligned}
$$

$\mathrm{RR} \mathrm{n}^{\circ} 8225$ 
We obtain that $m^{\star}=\pi(U[1])$ is a unimodular element of $M$, where $\pi: A^{1 \times 3} \longrightarrow M$ is the canonical projection onto $M$, and $\varphi \in \operatorname{hom}_{A}(M, A)$, defined by $\varphi(\pi(\lambda))=\lambda U[2]$ for all $\lambda \in A^{1 \times 3}$, satisfies $\varphi\left(m^{\star}\right)=1$ :

$>\operatorname{Mult}(\mathrm{U}[1], \mathrm{U}[2], \mathrm{A})$;

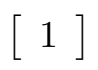

Let us check that $\varphi \in \operatorname{hom}_{D}(M, D)$, i.e., $R U[2]=0$ :

$>\operatorname{Mult}(\mathrm{R}, \mathrm{U}[2], \mathrm{A})$;

$$
[0]
$$

Since $M$ admits a unimodular element, $M$ can be decomposed as $M \cong A \oplus M^{\prime}$. A presentation of the left $A$-module $M^{\prime}$ can be obtained using the command FreeDirectSummand with the option "presentation".

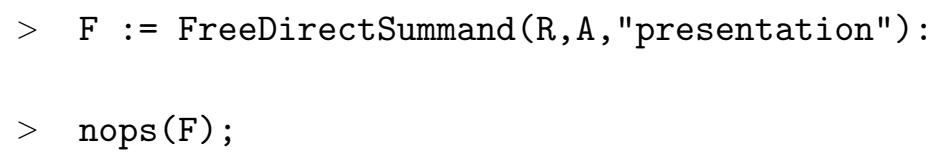

The output of the command FreeDirectSummand with the option "presentation" is a list with four entries. The first one $F[1]$ is a representative of a unimodular element of $M$

$>\mathrm{F}[1]$;

$$
\left[\begin{array}{lll}
0 & 1 & 0
\end{array}\right]
$$

i.e., $m^{\star}=\pi(F[1])$ is a unimodular element of $M$. The second entry of $F$

$>\mathrm{F}[2]$;

$$
\left[\begin{array}{c}
-\left(d_{3}+x_{1}+d_{2}\right)\left(d_{3}+x_{1}\right) \\
1 \\
2+d_{3} d_{1}+d_{3} x_{2}+x_{1} d_{1}+x_{1} x_{2}+d_{1} d_{2}+x_{2} d_{2}
\end{array}\right]
$$

defines $\varphi \in \operatorname{hom}_{A}(M, A)$ by $\varphi(\pi(\lambda))=\lambda F[2]$ for all $\lambda \in A^{1 \times 3}$, which satisfies $\varphi\left(m^{\star}\right)=1$. The third entry of $F$, namely,

$>\mathrm{F}[3]$;

$$
\left[\begin{array}{ccc}
1 & 0 & 0 \\
-1 & d_{1}+x_{2} & -d_{3}-x_{1}
\end{array}\right]
$$

is a presentation matrix of $M^{\prime}$, i.e., $M^{\prime}=A^{1 \times 3} /\left(A^{1 \times 2} F[3]\right)$ is such that $M^{\prime} \cong \operatorname{ker} \varphi$. Finally, the last entry of $F$, namely,

$\operatorname{RR} n^{\circ} 8225$ 
$>\mathrm{F}[4]$;

$$
\left[\begin{array}{ccc}
d_{1}+x_{2} & d_{2} & d_{3}+x_{1} \\
1 & d_{3}^{2}+2 d_{3} x_{1}+x_{1}^{2}+d_{2} d_{3}+d_{2} x_{1} & 0 \\
0 & 2+d_{3} d_{1}+d_{3} x_{2}+x_{1} d_{1}+x_{1} x_{2}+d_{1} d_{2}+x_{2} d_{2} & -1
\end{array}\right]
$$

defines an injective left $A$-homomorphism $i: M^{\prime} \longrightarrow M$ given by $i(\kappa(\gamma))=\pi(\gamma F[4])$ for all $\gamma \in A^{1 \times 3}$, where $\kappa: A^{1 \times 3} \longrightarrow M^{\prime}$ is the canonical projection onto $M^{\prime}$. Using the OreMorPHISMS package $([14])$, we can check again that $i$ is injective:

$>\operatorname{TestInj}(F[3], R, F[4], A)$;

true

Let us compute $\operatorname{rank}_{A}\left(M^{\prime}\right)$ :

$>\operatorname{OreRank}(\mathrm{F}[3], \mathrm{A})$;

Since $\operatorname{rank}_{A}\left(M^{\prime}\right)=1$, we cannot use Serre's splitting-off theorem again to decompose the left $A$-module $M^{\prime}$.

Using the option "isomorphism" of the command FreeDirectSummand,

$>$ G := FreeDirectSummand (R,A,"isomorphism"):

we can get another representation of the above splitting. The output $G$ contains

$>\operatorname{nops}(G)$;

four entries. The first one

$>\mathrm{G}[1]$;

$$
\left[\begin{array}{lll}
0 & 1 & 0
\end{array}\right]
$$

defines the unimodular element $m^{\star}=\pi(G[1])$ of $M$. The second one, namely,

$>\mathrm{G}[2]$;

$$
\left[\begin{array}{c}
-\left(d_{3}+x_{1}+d_{2}\right)\left(d_{3}+x_{1}\right) \\
1 \\
2+d_{3} d_{1}+d_{3} x_{2}+x_{1} d_{1}+x_{1} x_{2}+d_{1} d_{2}+x_{2} d_{2}
\end{array}\right]
$$

defines $\varphi \in \operatorname{hom}_{A}(M, A)$ such that $\varphi\left(m^{\star}\right)=1$. The third one

$>\mathrm{G}[3]$;

$\mathrm{RR} \mathrm{n}^{\circ} 8225$ 


$$
\left[\begin{array}{cccc}
0 & 1 & 0 & 0 \\
0 & -1 & d_{1}+x_{2} & -d_{3}-x_{1}
\end{array}\right]
$$

is a presentation matrix of the left $A$-module $\bar{M}=A^{1 \times 4} /\left(A^{1 \times 2} G[3]\right)$ which is such that $\bar{M} \cong A \oplus M^{\prime} \cong M$. Indeed, we note that $G[3]=\left(\begin{array}{ll}0 & F[3]\end{array}\right)$. The last entry of $G$

$>\mathrm{G}[4]$;

$$
\left[\begin{array}{ccc}
0 & 1 & 0 \\
d_{1}+x_{2} & d_{2} & d_{3}+x_{1} \\
1 & d_{3}^{2}+2 d_{3} x_{1}+x_{1}^{2}+d_{2} d_{3}+d_{2} x_{1} & 0 \\
0 & 2+d_{3} d_{1}+d_{3} x_{2}+x_{1} d_{1}+x_{1} x_{2}+d_{1} d_{2}+x_{2} d_{2} & -1
\end{array}\right]
$$

defines a left $A$-isomorphism $g: \bar{M} \longrightarrow M$, i.e., $g(\bar{\pi}(\theta))=\pi(\theta G[4])$ for all $\theta \in A^{1 \times 4}$, which is a left $A$-isomorphism. This last result can be checked again using the OreMorphIsms package ([14]):

$>$ TestIso(G[3],R,G[4],A);

true

We can simplify the presentation $G[3]$ of $\bar{M}$. Indeed, we have $\bar{M} \cong \underline{M}=A^{1 \times 3} /(A S[2])$, where $S[2]$ is the second entry of $S$ defined by:

$$
\begin{aligned}
& >\text { with(PurityFiltration): } \\
& >\mathrm{S}:=\text { ReducedPresentation (G[3],A); } \\
& \left.S:=\left[\begin{array}{cccc}
0 & 1 & 0 & 0 \\
0 & -1 & d_{1}+x_{2} & -d_{3}-x_{1}
\end{array}\right],\left[\begin{array}{ccc}
0 & d_{1}+x_{2} & -d_{3}-x_{1}
\end{array}\right],\left[\begin{array}{ccc}
1 & 0 & 0 \\
0 & 0 & 0 \\
0 & 1 & 0 \\
0 & 0 & 1
\end{array}\right],\left[\begin{array}{cccc}
1 & 0 & 0 & 0 \\
0 & 0 & 1 & 0 \\
0 & 0 & 0 & 1
\end{array}\right]\right]
\end{aligned}
$$

The left $A$-isomorphism $h: \underline{M} \longrightarrow \bar{M}$ is defined by $h(\sigma(\nu))=\bar{\pi}(\nu S[4])$ for all $\nu \in A^{1 \times 3}$, where $\sigma: A^{1 \times 3} \longrightarrow \underline{M}$ is the canonical projection onto $\underline{M}$, and $h^{-1}(\bar{\pi}(\theta))=\sigma(\theta S[3])$ for all $\theta \in A^{1 \times 4}$.

$$
>\text { TestIso(S[2],S[1],S[4],A); }
$$

Hence, if we define $P=S[4] G[4]$, namely,

$$
>P:=\operatorname{Mult}(\mathrm{S}[4], \mathrm{G}[4], \mathrm{A}) \text {; }
$$




$$
P:=\left[\begin{array}{ccc}
0 & 1 & 0 \\
1 & d_{3}^{2}+2 d_{3} x_{1}+x_{1}{ }^{2}+d_{2} d_{3}+d_{2} x_{1} & 0 \\
0 & 2+d_{3} d_{1}+d_{3} x_{2}+x_{1} d_{1}+x_{1} x_{2}+d_{1} d_{2}+x_{2} d_{2} & -1
\end{array}\right]
$$

then $i=g \circ h: \underline{M} \longrightarrow M$ defined by $i(\sigma(\nu))=\pi(\nu P)$ for all $\nu \in A^{1 \times 3}$ is a left $A$-isomorphism.

$$
>\text { TestIso(S[2], R, P, A); }
$$

true

Let us now compute $i^{-1}: M \longrightarrow \underline{M}$.

$$
\begin{aligned}
>Q & :=\operatorname{InverseMorphism}(\mathrm{S}[2], \mathrm{R}, \mathrm{P}, \mathrm{A}) ; \\
Q & \left.:=\left[\begin{array}{ccc}
-d_{3}{ }^{2}-2 x_{1} d_{3}-x_{1}{ }^{2}-d_{2} d_{3}-x_{1} d_{2} & 1 & 0 \\
1 & 0 & 0 \\
2+d_{1} d_{3}+x_{2} d_{3}+x_{1} d_{1}+x_{1} x_{2}+d_{1} d_{2}+x_{2} d_{2} & 0 & -1
\end{array}\right],[1]\right]
\end{aligned}
$$

We obtain that $i^{-1}(\pi(\lambda))=\sigma(\lambda Q[1])$ for all $\lambda \in A^{1 \times 3}$. Finally, let us check again that $i^{-1}$ defines a left $A$-isomorphism between $M$ and $\underline{M}$.

$>\operatorname{TestIso}(\mathrm{R}, \mathrm{S}[2], \mathrm{Q}[1], \mathrm{A})$;

true

Example 21. Let us consider the second Weyl algebra $A=A_{2}(\mathbb{Q})$

$>A:=\operatorname{DefineOreAlgebra}(\operatorname{diff}=[\mathrm{dx}, \mathrm{x}]$, diff $=[\mathrm{dy}, \mathrm{y}], \operatorname{polynom}=[\mathrm{x}, \mathrm{y}])$ :

and the left $A$-module $M=A^{1 \times 6} /\left(A^{1 \times 3} R\right)$ finitely presented by the matrix $R$ defined by:

$>\mathrm{R}:=\operatorname{evalm}([[\mathrm{dx}, \mathrm{dy}, 0,0,0,0],[0,1,-1,0, \mathrm{dx}, \mathrm{dy}],[0,0, \mathrm{dx}, \mathrm{dy}, 0,0]])$;

$$
R:=\left[\begin{array}{cccccc}
d x & d y & 0 & 0 & 0 & 0 \\
0 & -1 & 1 & 0 & d x & d y \\
0 & 0 & d x & d y & 0 & 0
\end{array}\right]
$$

The left $A$-module $M$ corresponds to Cosserat's equations appearing in linear elasticity. See Example 6. The rank of $M$ is:

$>\operatorname{OreRank}(\mathrm{R}, \mathrm{A})$;

Thus, Theorem 7 shows that $M$ admits a unimodular element. Let us compute one:

$>\mathrm{U}:=$ UnimodularElement $(\mathrm{R}, \mathrm{A})$;

$\mathrm{RR} \mathrm{n}^{\circ} 8225$ 


$$
\left.U:=\left[\begin{array}{llllll}
0 & 0 & 0 & 0 & -1 & 0
\end{array}\right],\left[\begin{array}{c}
d y \\
-d x \\
0 \\
0 \\
-1 \\
0
\end{array}\right]\right]
$$

If $\pi: A^{1 \times 6} \longrightarrow M$ is the canonical projection onto $M$, then $m_{1}^{\star}=\pi(U[1])$ is a unimodular element of $M$. Moreover, $\varphi_{1} \in \operatorname{hom}_{A}(M, A)$ defined by $\varphi_{1}(\pi(\lambda))=\lambda U[2]$ for all $\lambda \in A^{1 \times 6}$ satisfies $\varphi_{1}\left(m_{1}^{\star}\right)=U[1] U[2]=1$ :

$>\operatorname{Mult}(\mathrm{U}[1], \mathrm{U}[2], \mathrm{A})$;

$$
[1]
$$

Let us check that $\varphi_{1} \in \operatorname{hom}_{A}(M, A)$, i.e., $U[2] \in \operatorname{ker}_{A}(R$.):

$>\operatorname{Mult}(\mathrm{R}, \mathrm{U}[2], \mathrm{A})$;

$$
\left[\begin{array}{l}
0 \\
0 \\
0
\end{array}\right]
$$

Therefore, we have $M=A m_{1}^{\star} \oplus \operatorname{ker} \varphi_{1}$. The left $A$-submodule $\operatorname{ker} \varphi_{1}$ of $M$ can be computed using the command FreeDirectSummand with the option "kernel".

$$
\begin{aligned}
& >\mathrm{E}:=\text { FreeDirectSummand }(\mathrm{R}, \mathrm{A}, \mathrm{\text {"kernel }} \text { ") } \\
& \left.E:=\left[\begin{array}{llllll}
0 & 0 & 0 & 0 & -1 & 0
\end{array}\right],\left[\begin{array}{c}
d y \\
-d x \\
0 \\
0 \\
-1 \\
0
\end{array}\right],\left[\begin{array}{cccccc}
1 & 0 & 0 & 0 & d y & 0 \\
0 & 0 & -1 & 0 & 0 & -d y \\
0 & 0 & 1 & 0 & 0 & 0 \\
0 & 0 & 0 & 1 & 0 & 0 \\
0 & 0 & 0 & 0 & 0 & 1
\end{array}\right]\right]
\end{aligned}
$$

The first entry $E[1]$ of $E$ corresponds to $U[1]$ and the second entry $E[2]$ is $U[2]$. The residue classes of the rows of the third entry $E[3]$ of $E$ generate $\operatorname{ker} \varphi_{1}$, i.e., $\operatorname{ker} \varphi_{1}=\sum_{i=1}^{5} A \pi\left(F[3]_{i \bullet}\right)$, where $F[3]_{i \bullet}$ is the $i^{\text {th }}$ row of $F[3]$.

The command FreeDirectSummand with the option "presentation" computes a presentation of $\operatorname{ker} \varphi_{1}$.

$\mathrm{RR} \mathrm{n}^{\circ} 8225$ 
$>F:=$ FreeDirectSummand (R,A, "presentation");

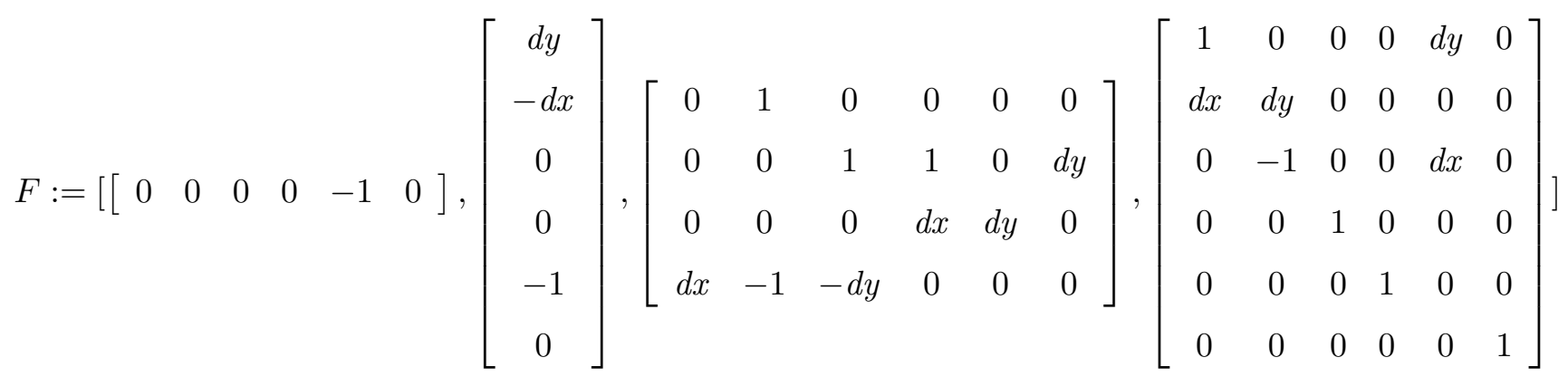

The first entry $F[1]$ of $F$ corresponds to $U[1]$ and the second entry $F[2]$ of $F$ is $U[2]$. The third entry $F[3]$ of $F$ is a presentation of $\operatorname{ker} \varphi_{1}$, i.e., $\operatorname{ker} \varphi_{1} \cong O_{1}=A^{1 \times 6} /\left(A^{1 \times 4} F[3]\right)$. Finally, the left $A$-homomorphism $i_{1}: O_{1} \longrightarrow M$ is defined by $i_{1}\left(\kappa_{1}(\gamma)\right)=\pi(\gamma F[4])$ for all $\gamma \in A^{1 \times 6}$, where $\kappa_{1}: A^{1 \times 6} \longrightarrow O_{1}$ is the canonical projection onto $O_{1}$. Let us check again that $i_{1}$ is injective:

$>\operatorname{TestInj}(\mathrm{F}[3], \mathrm{R}, \mathrm{F}[4], \mathrm{A})$;

true

Using the option "isomorphism" of the command FreeDirectSummand

$>$ F2 := FreeDirectSummand (R,A, "isomorphism");

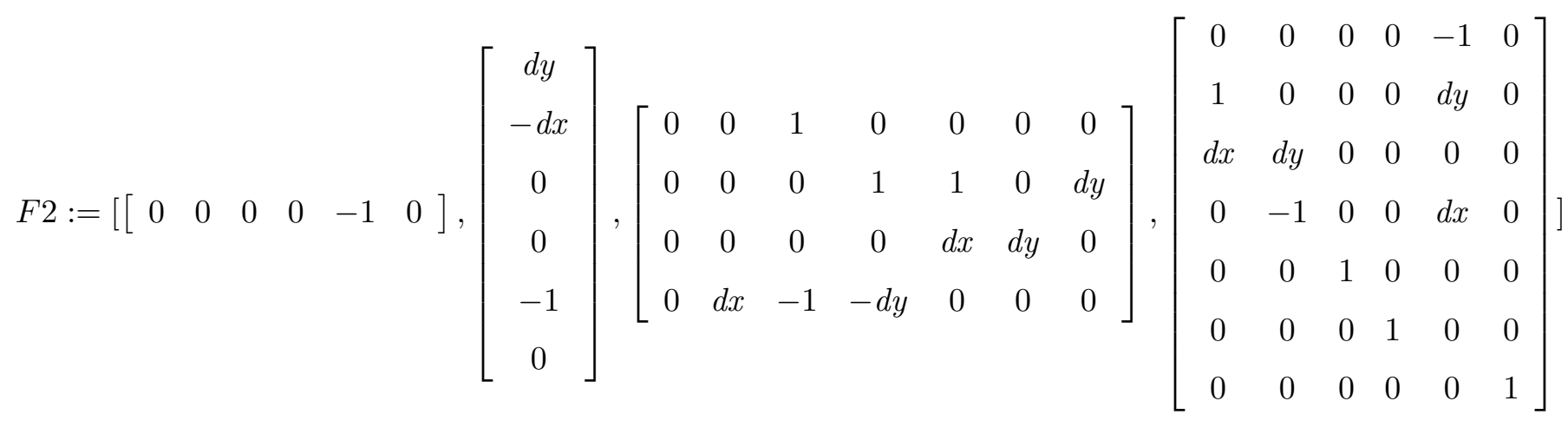

we first obtain a representative $F 2[1]$ of the unimodular element $m_{1}^{\star}=\pi(F 2[1])$ of $M$ and a form $\varphi_{1} \in \operatorname{hom}_{A}(M, A)$, defined $\varphi_{1}(\pi(\lambda))=\lambda F 2[2]$ for all $\lambda \in A^{1 \times 6}$, such that $\varphi_{1}\left(m_{1}^{\star}\right)=1$. Moreover, the left $A$-module $\bar{M}_{1}=A^{1 \times 7} /\left(A^{1 \times 4} F 2[3]\right)$ finitely presented by the third entry $F 2[3]$ of $F 2$ is such that $\bar{M}_{1} \cong A \oplus \operatorname{ker} \varphi_{1} \cong M$. The left $A$-homomorphism $g_{1}: \bar{M}_{1} \longrightarrow M$ defined by $g_{1}\left(\bar{\pi}_{1}(\theta)\right)=\pi(\theta F 2[4])$ for all $\theta \in A^{1 \times 7}$, where $F 2[4]$ is the fourth entry of $F 2$ and $\bar{\pi}_{1}: A^{1 \times 7} \longrightarrow \bar{M}_{1}$ is the canonical projection onto $\bar{M}_{1}$, is a left $A$-isomorphism. Let us check again that $g_{1}$ is a left $A$-isomorphism:

$>$ TestIso(F2[3], R,F2[4],A);

true

$\mathrm{RR} \mathrm{n}^{\circ} 8225$ 
Let us compute the rank of the left $A$-module $\operatorname{ker} \varphi_{1}$ :

$>\operatorname{OreRank}(\mathrm{F}[3], \mathrm{A})$;

2

Since $\operatorname{rank}_{A}\left(\operatorname{ker} \varphi_{1}\right)=2$, we can apply Theorem 7 to the left $A$-module $\operatorname{ker} \varphi_{1}$.

$>$ G := FreeDirectSummand(F[3],A, "presentation");

$\left.G:=\left[\begin{array}{llllll}0 & 0 & 0 & 0 & 0 & -1\end{array}\right],\left[\begin{array}{c}0 \\ 0 \\ 0 \\ d y \\ -d x \\ -1\end{array}\right],\left[\begin{array}{cccccc}0 & 1 & 0 & 0 & 0 & 0 \\ 0 & 0 & 1 & 1 & 0 & 0 \\ 0 & 0 & 0 & 0 & 1 & 0 \\ d x & -1 & -d y & 0 & 0 & 0 \\ 0 & 0 & 0 & d x & -1 & -d y\end{array}\right],\left[\begin{array}{cccccc}1 & 0 & 0 & 0 & 0 & 0 \\ 0 & 1 & 0 & 0 & 0 & 0 \\ 0 & 0 & 1 & 0 & 0 & 0 \\ 0 & 0 & 0 & 1 & 0 & d y \\ 0 & 0 & 0 & d x & d y & 0 \\ 0 & 0 & 0 & 0 & -1 & d x\end{array}\right]\right]$

We have $\operatorname{ker} \varphi_{1}=A m_{2}^{\star} \oplus \operatorname{ker} \varphi_{2}$, where $m_{2}^{\star}=\bar{\pi}_{1}(G[1])$ is a unimodular element of $\operatorname{ker} \varphi_{1}$ and $\varphi_{2} \in \operatorname{hom}_{A}\left(\operatorname{ker} \varphi_{1}, A\right)$, defined by $\varphi_{2}\left(\bar{\pi}_{1}(\zeta)\right)=\zeta G[2]$ for all $\zeta \in A^{1 \times 6}$, satisfies $\varphi_{2}\left(m_{2}^{\star}\right)=$ $G[1] G[2]=1$. Moreover, we have $\operatorname{ker} \varphi_{2} \cong O_{2}=A^{1 \times 6} /\left(A^{1 \times 5} G[3]\right)$ and the embeeding $i_{2}: O_{2} \longrightarrow \operatorname{ker} \varphi_{1}$ is defined by $i_{2}\left(\kappa_{2}(\nu)\right)=\nu G[4]$ for all $\nu \in A^{1 \times 7}$, where $\kappa_{2}: A^{1 \times 6} \longrightarrow O_{2}$ is the canonical projection onto $\mathrm{O}_{2}$. Let us check again that $i_{2}$ is injective:

$>\operatorname{TestInj}(\mathrm{G}[3], \mathrm{F}[3], \mathrm{G}[4], \mathrm{A})$;

true

Now, the rank of $\mathrm{O}_{2}$ is equal to:

$>\operatorname{OreRank}(\mathrm{G}[3], \mathrm{A})$;

1

Hence, Theorem 7 cannot be applied again to the left $A$-module $\operatorname{ker} \varphi_{2}$.

The left $A$-homomorphism $\iota=i_{2} \circ i_{1}: O_{2} \longrightarrow M$ is then defined by $\iota\left(\kappa_{2}(\nu)\right)=\nu J$ for all $\nu \in A^{1 \times 7}$, where $J=G[4] F[4]$ is defined by:

$>\mathrm{J}:=\operatorname{Mult}(\mathrm{G}[4], \mathrm{F}[4], \mathrm{A})$;

$$
J:=\left[\begin{array}{cccccc}
1 & 0 & 0 & 0 & d y & 0 \\
d x & d y & 0 & 0 & 0 & 0 \\
0 & -1 & 0 & 0 & d x & 0 \\
0 & 0 & 1 & 0 & 0 & d y \\
0 & 0 & d x & d y & 0 & 0 \\
0 & 0 & 0 & -1 & 0 & d x
\end{array}\right]
$$

$\mathrm{RR} \mathrm{n}^{\circ} 8225$ 
Let us check again that $\iota$ is injective:

$>\operatorname{TestInj}(\mathrm{G}[3], \mathrm{R}, \mathrm{J}, \mathrm{A})$;

true

Elementary operations can be used to simplify the presentation $G[3]$ of $\mathrm{O}_{2}$.

$>$ with(PurityFiltration):

$>\mathrm{K}:=$ ReducedPresentation $(\mathrm{G}[3], \mathrm{A})$;

$\left.K:=\left[\begin{array}{cccccc}0 & 1 & 0 & 0 & 0 & 0 \\ 0 & 0 & 1 & 1 & 0 & 0 \\ 0 & 0 & 0 & 0 & 1 & 0 \\ d x & -1 & -d y & 0 & 0 & 0 \\ 0 & 0 & 0 & d x & -1 & -d y\end{array}\right],\left[\begin{array}{ccc}d x & 0 & -d y \\ d y & d x & 0\end{array}\right],\left[\begin{array}{ccc}0 & 1 & 0 \\ 0 & 0 & 0 \\ -1 & 0 & 0 \\ 1 & 0 & 0 \\ 0 & 0 & 0 \\ 0 & 0 & 1\end{array}\right],\left[\begin{array}{cccccc}0 & 0 & 0 & 1 & 0 & 0 \\ 1 & 0 & 0 & 0 & 0 & 0 \\ 0 & 0 & 0 & 0 & 0 & 1\end{array}\right]\right]$

We obtain that $S=K[2]$ defined by

$>\mathrm{S}:=\mathrm{K}[2]$

$$
S:=\left[\begin{array}{ccc}
d x & 0 & -d y \\
d y & d x & 0
\end{array}\right]
$$

is a presentation matrix of a left $A$-module $O_{2}^{\prime}=A^{1 \times 3} /\left(A^{1 \times 2} S\right)$ which is isomorphic to $O_{2}$. Moreover, if $\kappa_{2}^{\prime}: A^{1 \times 3} \longrightarrow O_{2}^{\prime}$ is the canonical projection onto $O_{2}^{\prime}$, then the left $A$ homomorphism $j_{2}: O_{2}^{\prime} \longrightarrow O_{2}$, defined by $j_{2}\left(\kappa_{2}^{\prime}(\xi)\right)=\kappa_{2}(\xi K[4])$ for all $\xi \in A^{1 \times 3}$, is a left $A$-isomorphism. Thus, we get $M \cong A^{1 \times 2} \oplus O_{2}^{\prime}$. The left $A$-homomorphism $\chi: O_{2}^{\prime} \longrightarrow M$ defined by $\chi\left(\kappa_{2}^{\prime}(\xi)\right)=\pi(\xi P)$ for all $\xi \in A^{1 \times 3}$, where $P=K[4] J$ is defined by

$>P:=\operatorname{Mult}(\mathrm{K}[4], \mathrm{J}, \mathrm{A})$;

$$
P:=\left[\begin{array}{cccccc}
0 & 0 & 1 & 0 & 0 & d y \\
1 & 0 & 0 & 0 & d y & 0 \\
0 & 0 & 0 & -1 & 0 & d x
\end{array}\right]
$$

is injective:

$>\operatorname{TestInj}(\mathrm{S}, \mathrm{R}, \mathrm{P}, \mathrm{A})$;

true

We note that the left $A$-module $O_{2}^{\prime}$ corresponds to the linear PD system defining the equilibrium of the symmetric stress tensor. Hence, if $\mathcal{F}$ is a left $A$-module, then

$$
\operatorname{ker}_{\mathcal{F}}(R .) \cong \mathcal{F}^{2} \oplus \operatorname{ker}_{\mathcal{F}}(S .)
$$

$\mathrm{RR} \mathrm{n}^{\circ} 8225$ 
which shows that the solution space of Cosserat's equations is isomorphic to the direct sum of $\mathcal{F}^{2}$ and the solutions of the classical linear PD system defining the equilibrium of the symmetric stress tensor. Since $x$ and $y$ do not appear in the coefficients of the unimodular elements and in their corresponding forms, the above results are also valid over the commutative polynomial ring $B=\mathbb{Q}\left[\partial_{x}, \partial_{y}\right]$ and for any $B$-module $\mathcal{F}$. For more details, see Example 6.

Using the command MaximalFreeDirectSummand, the decomposition $M \cong A^{1 \times 2} \oplus \mathrm{O}_{2}$ can be obtained in one step.

$>\mathrm{N}:=$ MaximalFreeDirectSummand(R,A, "presentation"):

The first entry $N[1]$ of $N$ returns two unimodular elements $m_{1}^{\star}$ and $m_{2}^{\star}$ of $M$ and the corresponding two forms.

$>\mathrm{N}[1]$;

$$
\left[\left[\left[\begin{array}{llllll}
0 & 0 & 0 & 0 & -1 & 0
\end{array}\right],\left[\begin{array}{c}
d y \\
-d x \\
0 \\
0 \\
-1 \\
0
\end{array}\right]\right],\left[\left[\begin{array}{llllll}
0 & 0 & 0 & 0 & 0 & -1
\end{array}\right],\left[\begin{array}{c}
0 \\
0 \\
d y \\
-d x \\
0 \\
-1
\end{array}\right]\right]\right]
$$

The second entry $N[2]$, namely,

$>\mathrm{N}[2]$;

$$
\left[\begin{array}{cccccc}
0 & 1 & 0 & 0 & 0 & 0 \\
0 & 0 & 1 & 1 & 0 & 0 \\
0 & 0 & 0 & 0 & 1 & 0 \\
d x & -1 & -d y & 0 & 0 & 0 \\
0 & 0 & 0 & d x & -1 & -d y
\end{array}\right]
$$

is a presentation matrix of the left $A$-module $O_{2}=A^{1 \times 6} /\left(A^{1 \times 5} N[2]\right) \cong \operatorname{ker} \varphi_{2}$. Moreover, the left $A$-homomorphism $i_{2}: O_{2} \longrightarrow M$ defined by $i_{2}\left(\kappa_{2}(\nu)\right)=\pi(\nu N[3])$ for all $\nu \in A^{1 \times 6}$, where the matrix $N[3]$ is given by

$>\mathrm{N}[3]$;

$\mathrm{RR} \mathrm{n}^{\circ} 8225$ 


$\left[\begin{array}{cccccc}1 & 0 & 0 & 0 & d y & 0 \\ d x & d y & 0 & 0 & 0 & 0 \\ 0 & -1 & 0 & 0 & d x & 0 \\ 0 & 0 & 1 & 0 & 0 & d y \\ 0 & 0 & d x & d y & 0 & 0 \\ 0 & 0 & 0 & -1 & 0 & d x\end{array}\right]$

is injective:

$>\operatorname{TestInj}(\mathrm{N}[2], \mathrm{R}, \mathrm{N}[3], \mathrm{A})$;

true

Since the rank of $\mathrm{O}_{2}$ is one

$>\operatorname{OreRank}(\mathrm{N}[2], \mathrm{A})$;

Theorem 7 cannot be applied again to decompose $\mathrm{O}_{2}$.

If we use the option "isomorphism" of the command MaximalFreeDirectSummand,

$>$ N2 := MaximalFreeDirectSummand(R,A,"isomorphism"):

then we first find again two unimodular elements $m_{1}^{\star}$ and $m_{2}^{\star}$ of $M$ with their corresponding forms $\varphi_{1}$ and $\varphi_{2}$,

$>\mathrm{N} 2[1]$;

$$
\left[\left[\left[\begin{array}{llllll}
0 & 0 & 0 & 0 & -1 & 0
\end{array}\right],\left[\begin{array}{c}
d y \\
-d x \\
0 \\
0 \\
-1 \\
0
\end{array}\right]\right],\left[\left[\begin{array}{llllll}
0 & 0 & 0 & 0 & 0 & -1
\end{array}\right],\left[\begin{array}{c}
0 \\
0 \\
d y \\
-d x \\
0 \\
-1
\end{array}\right]\right]\right]
$$

and the left $A$-module $\bar{M}$ finitely presented by the second entry $N 2[2]$ of $N 2$, namely,

$>\mathrm{N} 2[2]$;

$\mathrm{RR} \mathrm{n}^{\circ} 8225$ 


$$
\left[\begin{array}{cccccccc}
0 & 0 & 0 & 1 & 0 & 0 & 0 & 0 \\
0 & 0 & 0 & 0 & 1 & 1 & 0 & 0 \\
0 & 0 & 0 & 0 & 0 & 0 & 1 & 0 \\
0 & 0 & d x & -1 & -d y & 0 & 0 & 0 \\
0 & 0 & 0 & 0 & 0 & d x & -1 & -d y
\end{array}\right]
$$

is isomorphic to $A^{1 \times 2} \oplus O_{2} \cong M$, i.e., $\bar{M}=A^{1 \times 8} /\left(A^{1 \times 5} N 2[2]\right) \cong A^{1 \times 2} \oplus O_{2} \cong M$. The left $A$-isomorphism $g: \bar{M} \longrightarrow M$ is defined by $g(\bar{\pi}(\theta))=\pi(\theta N 2[3])$ for all $\theta \in A^{1 \times 8}$, where the third entry $N 2[3]$ of $N 2$ is defined by:

$>\mathrm{N} 2[3]$;

$$
\left[\begin{array}{cccccc}
0 & 0 & 0 & 0 & -1 & 0 \\
0 & 0 & 0 & 0 & 0 & -1 \\
1 & 0 & 0 & 0 & d y & 0 \\
d x & d y & 0 & 0 & 0 & 0 \\
0 & -1 & 0 & 0 & d x & 0 \\
0 & 0 & 1 & 0 & 0 & d y \\
0 & 0 & d x & d y & 0 & 0 \\
0 & 0 & 0 & -1 & 0 & d x
\end{array}\right]
$$

We can check again that $g$ is a left $A$-isomorphism:

$>$ TestIso(N2[2], R, N2[3], A);

$$
\text { true }
$$

Let us compute $f=g^{-1}: M \longrightarrow \bar{M}$. We have $f(\pi(\lambda))=\bar{\pi}(\lambda X[1])$ for all $\lambda \in A^{1 \times 6}$, where $X[1]$ is the first entry of $X$ defined by:

$$
\begin{aligned}
& >\mathrm{X}:=\text { InverseMorphism }(\mathrm{N} 2[2], \mathrm{R}, \mathrm{N} 2[3], \mathrm{A}) \\
& \left.X:=\left[\begin{array}{cccccccc}
d y & 0 & 1 & 0 & 0 & 0 & 0 & 0 \\
-d x & 0 & 0 & 0 & 0 & 1 & 0 & 0 \\
0 & d y & 0 & 0 & 0 & 1 & 0 & 0 \\
0 & -d x & 0 & 0 & 0 & 0 & 0 & -1 \\
-1 & 0 & 0 & 0 & 0 & 0 & 0 & 0 \\
0 & -1 & 0 & 0 & 0 & 0 & 0 & 0
\end{array}\right],\left[\begin{array}{ccccc}
1 & d y & 0 & 1 & 0 \\
0 & 0 & 0 & 0 & 0 \\
0 & 0 & 1 & 0 & 1
\end{array}\right]\right]
\end{aligned}
$$


$>$ TestIso(R,N2[2], X[1],A);

true

Example 22. Let us consider the first Weyl algebra $A=A_{1}(\mathbb{Q})$

$>A:=$ DefineOreAlgebra $(\operatorname{diff}=[\mathrm{d}, \mathrm{t}]$, polynom $=[\mathrm{t}])$ :

and the left $A$-module $M=A^{1 \times 3} /(A R)$ finitely presented by the matrix $R$ defined by

$>\mathrm{R}:=\operatorname{evalm}([[\mathrm{d}, 0,-\mathrm{t}]])$;

$$
R:=\left[\begin{array}{lll}
d & 0 & -t
\end{array}\right]
$$

and the left $A$-module $M^{\prime}=A^{1 \times 6} /\left(A^{1 \times 3} R^{\prime}\right)$ finitely presented by the matrix $R^{\prime}$ defined by:

$>\operatorname{Rp}:=\operatorname{evalm}([[\mathrm{d},-\mathrm{t}, 0,0,0,-1],[0, \mathrm{~d}, 0,-\mathrm{t}, 0,0],[0,0, \mathrm{~d}, 0,-\mathrm{t}, 0]])$;

$$
R p:=\left[\begin{array}{cccccc}
d & -t & 0 & 0 & 0 & -1 \\
0 & d & 0 & -t & 0 & 0 \\
0 & 0 & d & 0 & -t & 0
\end{array}\right]
$$

For more details, see Example 7. Let us also consider the matrix $P \in A^{3 \times 6}$ defined by

$>P:=\operatorname{evalm}([[0,0,1,0,0,0],[1,0,0,0,0,1],[0,0,0,0,1,0]])$;

$$
P:=\left[\begin{array}{llllll}
0 & 0 & 1 & 0 & 0 & 0 \\
1 & 0 & 0 & 0 & 0 & 1 \\
0 & 0 & 0 & 0 & 1 & 0
\end{array}\right]
$$

and the matrix $P^{\prime} \in A^{1 \times 3}$ defined by

$>P p:=\operatorname{evalm}([[0,0,1]])$;

$$
P p:=\left[\begin{array}{lll}
0 & 0 & 1
\end{array}\right]
$$

which are such that $R P=P^{\prime} R^{\prime}$. Thus, they define the left $A$-homomorphism $\iota: M \longrightarrow M^{\prime}$ given by $\iota(\pi(\lambda))=\pi^{\prime}(\lambda P)$ for all $\lambda \in A^{1 \times 3}$, where $\pi: A^{1 \times 3} \longrightarrow M$ (resp., $\pi^{\prime}: A^{1 \times 6} \longrightarrow M^{\prime}$ ) is the canonical projection onto $M$ (resp., $M^{\prime}$ ). We can check that $\iota$ is injective:

$>\operatorname{TestInj}(\mathrm{R}, \mathrm{Rp}, \mathrm{P}, \mathrm{A})$;

$$
\text { true }
$$

Hence, we get $M \cong \iota(M)=\left(A^{1 \times 3} P+A^{1 \times 3} R^{\prime}\right) /\left(A^{1 \times 3} R^{\prime}\right) \subseteq M^{\prime}$.

Let us now compute an element $m^{\star} \in M$ such that $\iota\left(m^{\star}\right)$ is a unimodular element of $M^{\prime}$.

$>\mathrm{U}:=$ UnimodularElementInSubmodule (R, Rp, P, A):

$\mathrm{RR} \mathrm{n}^{\circ} 8225$ 
The output $U$ of the command UnimodularElementInSubmodule contains two entries

$>\operatorname{nops}(U)$;

the first one $U[1]$, namely,

$>\mathrm{U}[1]$;

$$
\left[\begin{array}{lll}
0 & 1 & 0
\end{array}\right]
$$

defines an element $m^{\star}=\pi(U[1])$ of $M$ which is such that $\iota\left(m^{\star}\right)$ is a unimodular element of $M^{\prime}$. The second entry $U[2]$ of $U$, namely,

$>\operatorname{map}(\operatorname{collect}, \mathrm{U}[2],[\mathrm{d}, \mathrm{t}])$;

$$
\left[\begin{array}{c}
-2 / 9 t^{2}+t-1 / 3 d t^{2}+\frac{2}{27} t^{3} \\
-1 / 3 t d^{2}+\left(1 / 3-5 / 9 t+\frac{2}{27} t^{2}\right) d+5 / 9+\frac{2}{27} t^{2} \\
0 \\
\frac{4}{27}-1 / 3 d^{3}+\left(-5 / 9+\frac{2}{27} t\right) d^{2}+\left(\frac{2}{27} t+\frac{4}{27}\right) d \\
0 \\
1 / 3 d t^{2}-t+1+2 / 9 t^{2}-\frac{2}{27} t^{3}
\end{array}\right]
$$

defines $\varphi \in \operatorname{hom}_{A}\left(M^{\prime}, A\right)$ which is such that $\varphi\left(\iota\left(m^{\star}\right)\right)=\varphi\left(\pi^{\prime}(U[1] P)\right)=U[1] P U[2]=1$. Indeed, if $\lambda^{\star}=U[1] P$, i.e.,

$>$ lambda_star := Mult $(\mathrm{U}[1], \mathrm{P}, \mathrm{A})$;

$$
\left[\begin{array}{llllll}
1 & 0 & 0 & 0 & 0 & 1
\end{array}\right]
$$

then $\lambda^{\star} U[2]=U[1] P U[2]$ is equal to:

$>\operatorname{Mult}($ lambda_star, $\mathrm{U}[2], \mathrm{A})$;

$$
\text { [1] }
$$

Finally, let us check that $\varphi \in \operatorname{hom}_{A}\left(M^{\prime}, A\right)$, i.e., $R^{\prime} U[2]=0$ :

$>\operatorname{Mult}(\mathrm{Rp}, \mathrm{U}[2], \mathrm{A})$;

$$
\left[\begin{array}{l}
0 \\
0 \\
0
\end{array}\right]
$$

$\mathrm{RR} \mathrm{n}^{\circ} 8225$ 


\subsection{Stafford's reduction}

Example 23. Let us consider the third Weyl algebra $A=A_{3}(\mathbb{Q})$

$>A:=$ DefineOreAlgebra(diff $=[d[3], x[3]], \operatorname{diff}=[d[1], x[1]], \operatorname{diff}=[d[2], x[2]]$, $>$ polynom $=[\mathrm{x}[3], \mathrm{x}[1], \mathrm{x}[2]])$ :

and the left $A$-module $L=A^{1 \times 3} /\left(A^{1 \times 3} P\right)$ finitely presented by the matrix $P$ of $\mathrm{PD}$ operators defining the curl operator in $\mathbb{R}^{3}$, namely:

$>P:=\operatorname{evalm}([[0,-d[3], d[2]],[d[3], 0,-d[1]],[-d[2], d[1], 0]]) ;$

$$
P:=\left[\begin{array}{ccc}
0 & -d_{3} & d_{2} \\
d_{3} & 0 & -d_{1} \\
-d_{2} & d_{1} & 0
\end{array}\right]
$$

Let us compute the rank of $L$ :

$>\operatorname{OreRank}(\mathrm{P}, \mathrm{A})$;

Thus, we get $\operatorname{rank}_{A}(L)=1$. We can check that $L$ is not a free left $A$-module of rank 1 since $\operatorname{ext}_{A}^{3}(N, A) \cong A /\left(A d_{1}+A d_{2}+A d_{3}\right) \neq 0$, where $N=A^{3} /\left(P A^{3}\right)$ is the Auslander transpose of $L$ :

$>\operatorname{Exti}(\operatorname{Involution}(\mathrm{P}, \mathrm{A}), \mathrm{A}, 2)$;

$$
\left.\left[\begin{array}{l}
d_{2} \\
d_{1} \\
d_{3}
\end{array}\right],[1], S U R J(1)\right]
$$

For more details, see [11]. Equivalently, we can check that no generalized inverse of $P$ exists:

$>$ GeneralizedInverse $(\mathrm{P}, \mathrm{A})$;

Lemma 5 then shows that $L$ can be generated by two elements. Using Stafford's reduction, let us try to compute a presentation of $L$ with two generators:

$>\mathrm{S}:=\operatorname{map}(\operatorname{collect}, \operatorname{StaffordReduction}(\mathrm{P}, \mathrm{A}),[\mathrm{d}[1], \mathrm{d}[2], \mathrm{d}[3]])$ :

The output of the command StaffordReduction is a list with two entries.

$$
>\operatorname{nops}(\mathrm{S}) \text {; }
$$

The first entry $S[1]$ of $S$

$\mathrm{RR} \mathrm{n}^{\circ} 8225$ 
$>\mathrm{S}[1] ;$
$\left.\qquad \begin{array}{cc}0 & 0 \\ -d_{3}{ }^{2} & -d_{1} d_{3}+\left(2+d_{3}\left(x_{3}+1\right)\right) d_{2} \\ d_{1} d_{3}+\left(1+\left(-x_{3}-1\right) d_{3}\right) d_{2} & d_{1}{ }^{2}+\left(-2-2 x_{3}\right) d_{2} d_{1}+\left(2 x_{3}+x_{3}{ }^{2}+1\right) d_{2}{ }^{2}\end{array}\right]$

is a matrix presenting a left $A$-module $\bar{L}=A^{1 \times 2} /\left(A^{1 \times 3} S[1]\right)$ isomorphic to $L$, i.e., $\bar{L} \cong L$. The second entry $S[2]$ of $S$ defines this left $A$-isomorphism

$>\mathrm{S}[2]$;

$$
\left[\begin{array}{ccc}
-1 & x_{3}+1 & 0 \\
0 & 0 & 1
\end{array}\right]
$$

i.e., $\gamma: \bar{L} \longrightarrow L$ defined by $\gamma(\sigma(\theta))=\tau(\theta S[2])$ for all $\theta \in A^{1 \times 2}$, where $\sigma: A^{1 \times 2} \longrightarrow \bar{L}$ (resp., $\tau: A^{1 \times 3} \longrightarrow L$ ) is the canonical projection onto $\bar{L}$ (resp., $L$ ), is a left $A$-isomorphism:

$>$ TestIso(S[1],P,S[2],A);

true

A minimal set of generators of $L$ is defined by $\left\{z_{1}=\gamma\left(\sigma\left(\left(\begin{array}{ll}1 & 0\end{array}\right)\right)\right), z_{2}=\gamma\left(\sigma\left(\left(\begin{array}{ll}0 & 1\end{array}\right)\right)\right)\right\}$, i.e.,

$$
z_{1}=-y_{1}+\left(x_{3}+1\right) y_{2}, \quad z_{2}=y_{3},
$$

where $\left\{y_{j}=\tau\left(f_{j}\right)\right\}_{j=1,2,3}$ is a set of generators of $L$, where $\left\{f_{j}\right\}_{j=1,2,3}$ is the standard basis of $A^{1 \times 3}$. Moreover, computing $\gamma^{-1}$,

$$
\begin{aligned}
>\mathrm{U} & :=\operatorname{InverseMorphism}(\mathrm{S}[1], \mathrm{P}, \mathrm{S}[2], \mathrm{A}) ; \\
U & \left.:=\left[\begin{array}{cc}
d_{3} x_{3}+d_{3}-1 & -2 d_{2} x_{3}-d_{2} x_{3}^{2}-d_{2}+d_{1} x_{3}+d_{1} \\
d_{3} & -d_{2} x_{3}-d_{2}+d_{1} \\
0 & 1
\end{array}\right],\left[\begin{array}{ccc}
0 & 1 & 0 \\
0 & -x_{3}-1 & 0 \\
0 & 0 & 1
\end{array}\right]\right]
\end{aligned}
$$

we obtain that $\gamma^{-1}: L \longrightarrow \bar{L}$ is defined by $\gamma^{-1}(\tau(\lambda))=\sigma(\lambda U[1])$ for all $\lambda \in A^{1 \times 3}$. Thus, in terms of generators, we get $y=U[1] z$, where $y=\left(\begin{array}{lll}y_{1} & y_{2} & y_{3}\end{array}\right)^{T}$ and $z=\left(\begin{array}{ll}z_{1} & z_{2}\end{array}\right)^{T}$.

Let us try to reduce the number of relations of the above presentation of $\bar{L}$.

$>\mathrm{T}:=\operatorname{map}($ collect, StaffordReduction(P, A, "reduce_relations"=true),
$>\quad[\mathrm{d}[1], \mathrm{d}[2], \mathrm{d}[3]]):$

We obtain that $\bar{L}=A^{1 \times 2} /\left(A^{1 \times 2} T[1]\right)$, where $T[1]$ is defined by

$>\mathrm{T}[1]$;

$\mathrm{RR} \mathrm{n}^{\circ} 8225$ 


$$
\left[\begin{array}{cc}
-d_{1} d_{3}+\left(-1+d_{3}\left(x_{3}+1\right)\right) d_{2} & -d_{1}{ }^{2}+\left(2+2 x_{3}\right) d_{2} d_{1}+\left(-1-2 x_{3}-x_{3}{ }^{2}\right) d_{2}{ }^{2} \\
-d_{3}{ }^{2} & -d_{1} d_{3}+\left(2+d_{3}\left(x_{3}+1\right)\right) d_{2}
\end{array}\right]
$$

is isomorphic to $L$, i.e., $\gamma: \bar{L} \longrightarrow L$ is defined by $\gamma(\sigma(\theta))=\tau(\theta T[2])$ for all $\theta \in A^{1 \times 2}$, where $T[2]$ is defined by

$>\mathrm{T}[2]$;

$$
\left[\begin{array}{ccc}
-1 & x_{3}+1 & 0 \\
0 & 0 & 1
\end{array}\right]
$$

i.e., $T[2]=S[2]$. To conclude, the linear PD system $P y=0$ defined by the curl operator in $\mathbb{R}^{3}$ is equivalent to the linear PD system $T[1]\left(\begin{array}{ll}z_{1} & z_{2}\end{array}\right)^{T}=0$ in two unknowns $z_{1}, z_{2}$ and defined by two equations.

Example 24. Let us consider the second Weyl algebra $A=A_{2}(\mathbb{Q})$

$>A:=\operatorname{DefineOreAlgebra}(\operatorname{diff}=[\mathrm{dx}, \mathrm{x}], \operatorname{diff}=[\mathrm{dy}, \mathrm{y}]$, polynom $=[\mathrm{x}, \mathrm{y}], \mathrm{comm}=[\mathrm{u}, \mathrm{rho}, \mathrm{c}])$ : and the left $A$-module $L=A^{1 \times 3} /\left(A^{1 \times 3} P\right)$ finitely presented by the matrix $P$ defined by:

$>\mathrm{P}:=\operatorname{Matrix}\left(3,3,\left[\left[\mathrm{u} * \mathrm{rho} * \mathrm{dx}, \mathrm{c}^{\wedge} 2 * \mathrm{dx}, 0\right],\left[0, \mathrm{c}^{\wedge} 2 * \mathrm{dy}, \mathrm{u} * \mathrm{rho} * \mathrm{dx}\right]\right.\right.$,

$>\quad[\mathrm{rho} * \mathrm{dx}, \mathrm{u} * \mathrm{dx}, \mathrm{rho} * \mathrm{dy}]])$;

$$
P:=\left[\begin{array}{ccc}
u \rho d x & c^{2} d x & 0 \\
0 & c^{2} d y & u \rho d x \\
\rho d x & u d x & \rho d y
\end{array}\right]
$$

The left $A$-module $L$ corresponds to the steady two-dimensional rotational isentropic flow (see, R. Courant, D. Hilbert, Methods of Mathematical Physics, Volume II, Wiley Classics Library, Wiley, 1962, pp. 436-437). Let us compute $\operatorname{ker}_{A}(. P)$ :

$$
>\operatorname{SyzygyModule}(\mathrm{P}, \mathrm{A}) \text {; }
$$

Thus, $P$ has full row rank, which shows that $\operatorname{rank}_{A}\left(A^{1 \times 3} P\right)=3$. Theorem 9 shows that $L$ admits Stafford's reduction. Let us compute one. We obtain

$>\mathrm{S}:=\operatorname{map}(\operatorname{collect}$, StaffordReduction $(\mathrm{P}, \mathrm{A}),[\mathrm{dx}, \mathrm{dy}]):$

$>\operatorname{nops}(\mathrm{S})$;

that $L \cong \bar{L}=A^{1 \times 2} /\left(A^{1 \times 3} S[1]\right)$ where $S[1]$ is defined by

$>\mathrm{S}[1]$;

$\mathrm{RR} \mathrm{n}^{\circ} 8225$ 


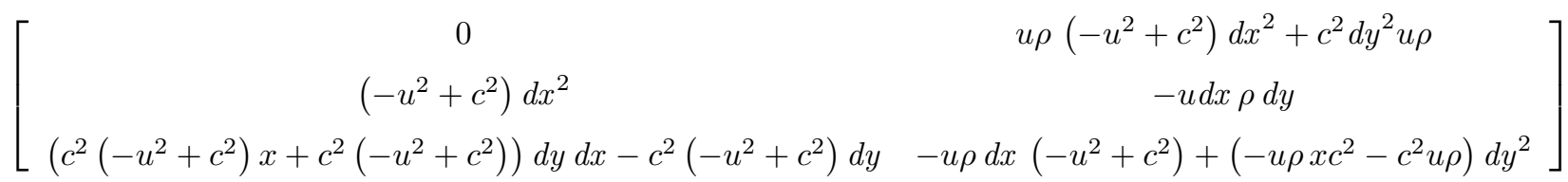

and the left $A$-isomorphism $\gamma: \bar{L} \longrightarrow L$ is defined $\gamma(\sigma(\theta))=\tau(\theta S[2])$ for all $\theta \in A^{1 \times 2}$, where $S[2]$ is defined by

$>\operatorname{map}(\operatorname{collect}, \mathrm{S}[2],[\mathrm{u}, \mathrm{rho}, \mathrm{c}]$, distributed);

$$
\left[\begin{array}{ccc}
(x+1) u \rho & 1+(x+1) c^{2} & 0 \\
0 & 0 & 1
\end{array}\right]
$$

and $\sigma: A^{1 \times 2} \longrightarrow \bar{L}$ (resp., $\tau: A^{1 \times 3} \longrightarrow L$ ) is the canonical projection onto $\bar{L}$ (resp., $L$ ). We can check again that $\gamma$ defines a left $A$-isomorphism:

$>$ TestIso(S[1],P,S[2],A):

true

If $\left\{f_{j}\right\}_{j=1,2,3}$ is the standard basis of $A^{1 \times 3}$, then $\left\{y_{j}=\tau\left(f_{j}\right)\right\}$ is a family of generators of $L$ (see Section 2). Then, $z=S[2] y$ is a set of generators $\left\{z_{1}, z_{2}\right\}$ of $L$, where $y=\left(\begin{array}{lll}y_{1} & y_{2} & y_{3}\end{array}\right)^{T}$ and $z=\left(\begin{array}{ll}z_{1} & z_{2}\end{array}\right)^{T}$. In particular, we have:

$$
z_{1}=u \rho(x+1) y_{1}+\left(c^{2}(x+1)+1\right) y_{2}, \quad z_{2}=y_{3} .
$$

The inverse $\gamma^{-1}: L \longrightarrow \bar{L}$ of $\gamma$ is defined by $\gamma^{-1}(\tau(\lambda))=\sigma(\lambda U[1])$ for all $\lambda \in A^{1 \times 3}$, where $U[1]$ is the first entry of $U$ defined by:

$$
\begin{aligned}
& >\quad \mathrm{U}:=\operatorname{map}(\text { collect, InverseMorphism }(\mathrm{S}[1], \mathrm{P}, \mathrm{S}[2], \mathrm{A}),[\mathrm{dx}, \mathrm{dy}, \mathrm{u}, \mathrm{rho}, \mathrm{c}], \\
& >\text { distributed); } \\
& \left.\left[\begin{array}{cc}
\frac{(x+1) c^{2} d x}{u \rho}-\frac{c^{2}}{u \rho}+\frac{d x}{u \rho} & -\frac{d y\left(1+(x+1) c^{2}\right)}{-u^{2}+c^{2}} \\
1+(-x-1) d x & \frac{u \rho d y(x+1)}{-u^{2}+c^{2}} \\
0 & 1
\end{array}\right],\left[\begin{array}{ccc}
0 & \left(-u^{2}+c^{2}\right)^{-1} & 0 \\
0 & 0 & -\left(-u^{2}+c^{2}\right)^{-1} \\
0 & \frac{1+(x+1) c^{2}+(-x-1) u^{2}}{u\left(-u^{2}+c^{2}\right)} & 0
\end{array}\right]\right]
\end{aligned}
$$

We can check again that $U[1]$ defines a left $A$-isomorphism from $L$ to $\bar{L}$.

$$
>\operatorname{TestIso}(\mathrm{P}, \mathrm{S}[1], \mathrm{U}[1], \mathrm{A}) \text {; }
$$

true

The above results show that $y=U[1] z$, and the linear $\mathrm{PD}$ system $P y=0$ is equivalent to the linear $\mathrm{PD}$ system $S[1] z=0$ defined by two unknowns and three equations.

Theorem 9 shows that one relation can be removed from $S[1]$, i.e., there exists $T \in A^{2 \times 2}$ such that $A^{1 \times 3} S[1]=A^{1 \times 2} T$, and thus $L$ is isomorphic to the left $A$-module $\bar{L}=A^{1 \times 2} /\left(A^{1 \times 2} T\right)$. Let us compute such a matrix $T$ :

$\mathrm{RR} \mathrm{n}^{\circ} 8225$ 


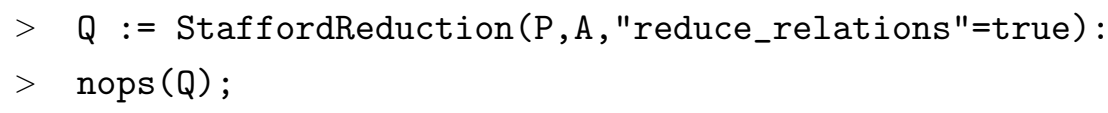

We can choose $T=Q[1]$, where:

$$
\begin{aligned}
& >\mathrm{Q}[1] ; \\
& {\left[\begin{array}{cc}
\left(c^{2}\left(-u^{2}+c^{2}\right) x+c^{2}\left(-u^{2}+c^{2}\right)\right) d y d x-c^{2}\left(-u^{2}+c^{2}\right) d y & -u \rho d x\left(-u^{2}+c^{2}\right)-u \rho c^{2}(x+1) d y^{2} \\
\left(u^{2}-c^{2}\right) d x^{2} & u d x \rho d y
\end{array}\right]}
\end{aligned}
$$

Moreover, we have $Q[2]=S[2]$

$>\operatorname{map}(\operatorname{collect}, \mathrm{Q}[2],[\mathrm{u}, \mathrm{c}, \mathrm{rho}])$;

$$
\left[\begin{array}{ccc}
(x+1) u \rho & 1+(x+1) c^{2} & 0 \\
0 & 0 & 1
\end{array}\right]
$$

which shows that the left $A$-isomorphism $\gamma: L \longrightarrow \bar{L}=A^{1 \times 2} /\left(A^{1 \times 2} T\right)$ is defined by $\gamma(\sigma(\theta))=\tau(\theta Q[2])$ for all $\theta \in A^{1 \times 2}$.

Example 25. Let us consider the third Weyl algebra $A=A_{3}(\mathbb{Q})$

$>A:=$ DefineOreAlgebra $(\operatorname{diff}=[\mathrm{d}[1], \mathrm{x}[1]], \operatorname{diff}=[\mathrm{d}[2], \mathrm{x}[2]], \operatorname{diff}=[\mathrm{d}[3], \mathrm{x}[3]]$, $>$ polynom $=[\mathrm{x}[1], \mathrm{x}[2], \mathrm{x}[3]]):$

and the left $A$-module $L=A^{1 \times 3} /\left(A^{1 \times 3} P\right)$ finitely presented by the matrix $P$ defined by:

$>\quad \mathrm{P}:=\operatorname{evalm}([[\mathrm{x}[2] * \mathrm{~d}[1] / 2, \mathrm{x}[2] * \mathrm{~d}[2]+1, \mathrm{x}[2] * \mathrm{~d}[3]+\mathrm{d}[1] / 2]$,

$>\quad[-\mathrm{x}[2] * \mathrm{~d}[2] / 2-3 / 2,0, \mathrm{~d}[2] / 2],[-\mathrm{d}[1]-\mathrm{x}[2] * \mathrm{~d}[3] / 2,-\mathrm{d}[2],-\mathrm{d}[3] / 2]])$;

$$
P:=\left[\begin{array}{ccc}
1 / 2 x_{2} d_{1} & x_{2} d_{2}+1 & x_{2} d_{3}+1 / 2 d_{1} \\
-1 / 2 x_{2} d_{2}-3 / 2 & 0 & 1 / 2 d_{2} \\
-d_{1}-1 / 2 x_{2} d_{3} & -d_{2} & -1 / 2 d_{3}
\end{array}\right]
$$

Let us compute its rank:

$>\operatorname{OreRank}(\mathrm{P}, \mathrm{A})$;

We obtain $\operatorname{rank}_{A}(L)=1$. Using 2 of Theorem $10, L$ is either a free left $A$-module of rank 1 or $L$ can be generated by two elements.

Let us compute Stafford's reduction of $L$ :

$>\mathrm{S}:=$ StaffordReduction(P,A, "reduce_generators"=true); 
$\left.S:=\left[\begin{array}{cc}-1 / 2 d_{1} x_{2} d_{2}-3 / 2 d_{1}-1 / 2 d_{3} x_{2}^{2} d_{2}-3 / 2 x_{2} d_{3} & 1 / 2 x_{2} d_{3} d_{2}+1 / 2 d_{2} d_{1} \\ -1 / 2 x_{2} d_{2}-3 / 2 & 1 / 2 d_{2}\end{array}\right],\left[\begin{array}{ccc}1 & 0 & 0 \\ 0 & 0 & 1\end{array}\right]\right]$

We obtain that the left $A$-homomorphism $\gamma: \bar{L}=A^{1 \times 2} /\left(A^{1 \times 2} S[1]\right) \longrightarrow L$ defined by $\gamma(\sigma(\theta))=\tau(\theta S[2])$ for all $\theta \in A^{1 \times 2}$, where $\sigma: A^{1 \times 2} \longrightarrow \bar{L}$ (resp., $\tau: A^{1 \times 3} \longrightarrow L$ ) is the canonical projection onto $\bar{L}$ (resp., $L$ ) is a left $A$-isomorphism. This result can be checked again:

$>$ TestIso(S[1],P,S[2],A);

true

Let us try to reduce the number of relations in the presentation matrix $S[1]$ of $\bar{L}$ :

$>\mathrm{T}:=$ StaffordReduction(S[1], A, "checkrank"=false, "reduce_generators"=true); $T:=\left[[0],\left[\begin{array}{ll}x_{2} & -1\end{array}\right]\right]$

We get $\bar{L} \cong A /(A T[1]) \cong A$. This last isomorphism can be checked again:

$>$ TestIso(T[1], S[1], T[2],A);

true

Thus, $L \cong \bar{L} \cong A$ is a free left $A$-module of rank 1 . If we define $U=T[2] S[2]$, i.e.,

$>\quad \mathrm{U}:=\operatorname{Mult}(\mathrm{T}[2], \mathrm{S}[2], \mathrm{A})$;

$$
U:=\left[\begin{array}{lll}
x_{2} & 0 & -1
\end{array}\right]
$$

then $\vartheta: A \longrightarrow L$ defined by $\vartheta(a)=\tau(a U)$ for all $a \in A$ is a left $A$-isomorphism:

$>$ TestIso(T[1],P,U,A);

true

In particular, since 1 is a basis of $A$, then $\vartheta(1)=\tau(U)$ is a basis of $L$.

Example 26. The next examples show that trivial reduction techniques can also be used to simplify the presentation of classical linear differential time-delay systems.

We first start with a wind tunnel model studied in A. Manitius, "Feedback controllers for a wind tunnel model involving a delay: analytical design and numerical simulations", IEEE Trans. Autom. Contr., 29 (1984), pp. 1058-1068.

Let us define the commutative polynomial ring $A=\mathbb{Q}(\zeta, k, a, \omega)[d, \delta]$

$>A:=$ DefineOreAlgebra (diff $=[d, t]$, dual_shift=[delta, $s]$, polynom= $[t, s]$, $>\quad c o m m=[z e t a, k, a, o m e g a]$, shift_action $=[$ delta, $t, h]):$

where $d y(t)=\dot{y}(t)$ is the differential operator and $\delta y(t)=y(t-h)$ is the time-delay operator. Let us also consider the matrix $P \in A^{3 \times 4}$ defining the wind tunnel model 
$>\mathrm{P}:=\operatorname{matrix}(3,4,[\mathrm{~d}+\mathrm{a}, \mathrm{k} * \mathrm{a} * \operatorname{delta}, 0,0,0, \mathrm{~d},-1,0,0$, omega`2, $\mathrm{d}+2 * z e t a *$ omega,
$>\quad$-omega`2]);

$$
P:=\left[\begin{array}{cccc}
d+a & k a \delta & 0 & 0 \\
0 & d & -1 & 0 \\
0 & \omega^{2} & d+2 \zeta \omega & -\omega^{2}
\end{array}\right]
$$

and the $A$-module $L=A^{1 \times 4} /\left(A^{1 \times 3} P\right)$. We can use Stafford's reduction to try to simplify the presentation of $L$ by removing trivial unimodular elements, namely, unimodular elements which are $A$-linear combinations of the generators of $L$ but not $A_{2}(\mathbb{Q}(\zeta, k, a, \omega))$-linear ones.

$>\mathrm{S}:=$ StaffordReduction (P,A,"reduce_relations"=true);

$$
\left.S:=\left[\begin{array}{ccc}
d+a & -\frac{k a \delta d}{\omega^{2}}-2 \frac{k a \zeta \delta}{\omega} & k a \delta \\
0 & \frac{d^{2}}{\omega^{2}}+2 \frac{\zeta d}{\omega}+1 & -d
\end{array}\right],\left[\begin{array}{cccc}
1 & 0 & 0 & 0 \\
0 & 0 & 1 & 0 \\
0 & 0 & 0 & 1
\end{array}\right]\right]
$$

We get that $L \cong \bar{L}=A^{1 \times 3} /\left(A^{1 \times 2} S[1]\right)$, where the $A$-isomorphism $\gamma: \bar{L} \longrightarrow L$ is defined by $\gamma(\sigma(\theta))=\tau(\theta S[2])$ for all $\theta \in A^{1 \times 3}$, where $\sigma: A^{1 \times 3} \longrightarrow \bar{L}$ (resp., $\tau: A^{1 \times 4} \longrightarrow L$ ) is the canonical projection onto $\bar{L}$ (resp., $L$ ).

$>$ TestIso(S[1],P,S[2], A);

true

We can try again to find a trivial reduction of the presentation of $\bar{L}$.

$$
\begin{aligned}
& >\mathrm{T}:=\text { StaffordReduction (S[1],A, "reduce_relations"=true); } \\
& T:=\left[\left[\begin{array}{ll}
d+a & -\frac{k a \delta}{\omega^{2}}
\end{array}\right],\left[\begin{array}{ccc}
1 & 0 & 0 \\
0 & d+2 \zeta \omega & -\omega^{2}
\end{array}\right]\right]
\end{aligned}
$$

Thus, $\bar{L} \cong \underline{L}=A^{1 \times 2} /(A T[1])$, where the $A$-isomorphism $\beta: \underline{L} \longrightarrow \bar{L}$ is defined by $\beta(\kappa(\xi))=$ $\sigma(\xi T[2])$ for all $\xi \in A^{1 \times 2}$, where $\kappa: A^{1 \times 2} \longrightarrow \underline{L}$ is the canonical projection onto $\underline{L}$.

$>$ TestIso(T[1],S[1], T[2], A);

true

If we define $U=T[2] S[2]$, namely,

$>\quad \mathrm{U}:=\operatorname{Mult}(\mathrm{T}[2], \mathrm{S}[2], \mathrm{A})$;

$$
U:=\left[\begin{array}{cccc}
1 & 0 & 0 & 0 \\
0 & 0 & d+2 \zeta \omega & -\omega^{2}
\end{array}\right]
$$

then $\vartheta=\gamma \circ \beta: \underline{L} \longrightarrow L$ defined by $\vartheta(\kappa(\xi))=\tau(\xi U)$ for all $\xi \in A^{1 \times 2}$ is an $A$-isomorphism: 


\section{$>$ TestIso(T[1], P,U, A);}

true

Hence, we get that the linear differential time-delay system $P y=0$ defining the wind tunnel model is equivalent to the single differential time-delay equation:

$$
\dot{x}(t)+a x(t)-\frac{k a}{\omega^{2}} u(t-h)=0 .
$$

Finally, $\vartheta^{-1}: L \longrightarrow \underline{L}$ is defined by $\vartheta^{-1}(\tau(\theta))=\kappa(\theta Q[2])$ for all $\theta \in A^{1 \times 4}$, where $Q[2]$ is the second entry of $Q$ defined by

$>\mathrm{Q}:=\operatorname{InverseMorphism}(\mathrm{T}[1], \mathrm{P}, \mathrm{U}, \mathrm{A})$;

$$
Q:=\left[\left[\begin{array}{cc}
1 & 0 \\
0 & -\omega^{-2} \\
0 & -\frac{d}{\omega^{2}} \\
0 & -\frac{\omega^{2}+d^{2}+2 d \zeta \omega}{\omega^{4}}
\end{array}\right],\left[\begin{array}{l}
1 \\
0 \\
0
\end{array}\right]\right]
$$

and we can finally check again that $\vartheta^{-1}$ is an $A$-isomorphism:

$>\operatorname{TestIso}(\mathrm{P}, \mathrm{T}[1], \mathrm{Q}[1], \mathrm{A})$;

true

Example 27. Let us consider the commutative polynomial $\operatorname{ring} A=\mathbb{Q}\left(\eta_{1}, \eta_{2}\right)\left[d, \sigma_{1}, \sigma_{2}\right]$ of differential time-delay operators

$>A:=$ DefineOreAlgebra(diff $=[\mathrm{d}, \mathrm{t}]$, dual_shift=[sigma[1],s1],

$>$ dual_shift=[sigma[2],s2],polynom=[t,s1,s2], comm=[eta[1], eta[2]],

$>$ shift_action=[sigma[1],t,h[1]], shift_action=[sigma[2],t,h[2]]):

where $d y(t)=\dot{y}(t)$ and $\sigma_{i} y(t)=y\left(t-h_{i}\right)$ for $i=1,2$, the matrix $P \in A^{4 \times 6}$ defined by

$>P:=\operatorname{evalm}([[1,1,-1,-1,0,0],[d+e t a[1], d-e t a[1],-e t a[2], \operatorname{eta}[2], 0,0]$,

$\left.\left.>\quad\left[\operatorname{sigma}[1]^{\wedge} 2,1,0,0,-\operatorname{sigma}[1], 0\right],[0,0,1, \operatorname{sigma}[2] ` 2,0,-\operatorname{sigma}[2]]\right]\right)$;

$$
P:=\left[\begin{array}{cccccc}
1 & 1 & -1 & -1 & 0 & 0 \\
d+\eta_{1} & d-\eta_{1} & -\eta_{2} & \eta_{2} & 0 & 0 \\
\sigma_{1}{ }^{2} & 1 & 0 & 0 & -\sigma_{1} & 0 \\
0 & 0 & 1 & \sigma_{2}^{2} & 0 & -\sigma_{2}
\end{array}\right]
$$

and the $A$-module $L=A^{1 \times 6} /\left(A^{1 \times 4} P\right)$ which defines a vibrating string with an interior mass considered in H. Mounier, J. Rudolph, M. Fliess, P. Rouchon, "Tracking control of a 
vibrating string with an interior mass viewed as delay system", ESAIM Control, Optimisation and Calculus of Variations, 3 (1998), pp. 315-321. Let us apply Stafford's reduction to L.

$$
\begin{aligned}
& >\mathrm{S}:=\text { StaffordReduction(P,A, "reduce_relations"=true); } \\
& \left.S:=\left[\begin{array}{ccccc}
-2 \eta_{1} & d+\eta_{1}-\eta_{2} & d+\eta_{1}+\eta_{2} & 0 & 0 \\
-1+\sigma_{1}{ }^{2} & -\sigma_{1}{ }^{2} & -\sigma_{1}{ }^{2} & \sigma_{1} & 0 \\
0 & 1 & \sigma_{2}{ }^{2} & 0 & -\sigma_{2}
\end{array}\right],\left[\begin{array}{cccccc}
0 & 1 & 0 & 0 & 0 & 0 \\
0 & 0 & 1 & 0 & 0 & 0 \\
0 & 0 & 0 & 1 & 0 & 0 \\
0 & 0 & 0 & 0 & 1 & 0 \\
0 & 0 & 0 & 0 & 0 & 1
\end{array}\right]\right]
\end{aligned}
$$

We find that $\bar{L}=A^{1 \times 5} /\left(A^{1 \times 3} S[1]\right) \cong L$, where $\gamma: \bar{L} \longrightarrow L$ defined by $\gamma(\sigma(\theta))=\tau(\theta S[2])$ for all $\theta \in A^{1 \times 5}$, where $\sigma: A^{1 \times 5} \longrightarrow \bar{L}$ (resp., $\tau: A^{1 \times 6} \longrightarrow L$ ) is the canonical projection onto $\bar{L}$ (resp., $L$ ), is an $A$-isomorphism:

$>$ TestIso(S[1], P, S[2],A);

\section{true}

Let us try to apply Stafford's reduction to the presentation of $\bar{L}$.

$>\mathrm{T}:=$ StaffordReduction(S[1], A, "reduce_relations"=true):

We obtain that $\bar{L} \cong \underline{L}=A^{1 \times 4} /\left(A^{1 \times 2} T[1]\right)$, where $T[1]$ is defined by

$$
\begin{aligned}
& >\mathrm{T}[1] ; \\
& {\left[\begin{array}{cccc}
-d+\left(-\eta_{1}-\eta_{2}\right) \sigma_{1}{ }^{2}+\sigma_{1}{ }^{2} d+\eta_{2}-\eta_{1} & \left(\eta_{2}-\eta_{1}\right) \sigma_{1}{ }^{2}+\sigma_{1}{ }^{2} d-\eta_{1}-\eta_{2}-d & 2 \eta_{1} \sigma_{1} & 0 \\
1 & \sigma_{2}{ }^{2} & 0 & -\sigma_{2}
\end{array}\right]}
\end{aligned}
$$

and $\beta: \underline{L} \longrightarrow \bar{L}$ defined by $\beta(\kappa(\xi))=\sigma(\xi T[2])$ for all $\xi \in A^{1 \times 4}$, where $T[2]$ is defined by

$$
>\mathrm{T}[2] \text {; }
$$

$$
\left[\begin{array}{lllll}
0 & 1 & 0 & 0 & 0 \\
0 & 0 & 1 & 0 & 0 \\
0 & 0 & 0 & 1 & 0 \\
0 & 0 & 0 & 0 & 1
\end{array}\right]
$$

and $\kappa: A^{1 \times 4} \longrightarrow \underline{L}$ is the canonical projection onto $\underline{L}$, is an $A$-isomorphism:

$>\operatorname{TestIso}(\mathrm{T}[1], \mathrm{S}[1], \mathrm{T}[2], \mathrm{A})$;

true

Let us try again to apply Stafford's reduction to $\underline{L}$ :

$\mathrm{RR} \mathrm{n}^{\circ} 8225$ 
$>\mathrm{U}:=\operatorname{map}(\operatorname{collect}$, StaffordReduction(T[1], A, "reduce_relations"=true),

$>$ [sigma [1], sigma [2],d], distributed):

$>\operatorname{rowdim}(\mathrm{U}[1]) ; \operatorname{coldim}(\mathrm{U}[1])$;

We obtain that $\underline{L} \cong L^{\prime}=A^{1 \times 3} /(A U[1])$, where $U[1]=(U[1][1,1] U[1][1,2] U[1][1,3])$ and

$>\mathrm{U}[1][1,1]$;

$$
\begin{array}{cc}
\left(-\eta_{1}-\eta_{2}\right) \sigma_{1}^{2} \sigma_{2}^{2}+\left(-\eta_{2}+\eta_{1}\right) \sigma_{1}^{2}+\left(\eta_{2}-\eta_{1}\right) \sigma_{2}^{2}+d \sigma_{1}^{2}{\sigma_{2}}^{2}+\eta_{2}+\eta_{1}+d-d \sigma_{2}{ }^{2}-\sigma_{1}{ }^{2} d \\
>\mathrm{U}[1][1,2] ; & -2 \eta_{1} \sigma_{1} \\
>\mathrm{U}[1][1,3] ; & \left(-\eta_{2}+\eta_{1}\right) \sigma_{2}-\sigma_{1}^{2} d \sigma_{2}+\left(\eta_{2}+\eta_{1}\right) \sigma_{1}^{2} \sigma_{2}+d \sigma_{2}
\end{array}
$$

and $\alpha: L^{\prime} \longrightarrow \underline{L}$ defined by $\alpha(v(\mu))=\kappa(\mu U[2])$ for all $\mu \in A^{1 \times 3}$, where $U[2]$ is defined by

$>\mathrm{U}[2]$;

$$
\left[\begin{array}{llll}
0 & 1 & 0 & 0 \\
0 & 0 & 1 & 0 \\
0 & 0 & 0 & 1
\end{array}\right]
$$

and $v: A^{1 \times 3} \longrightarrow L^{\prime}$ is the canonical projection onto $L^{\prime}$, is an $A$-isomorphism:

$$
>\text { TestIso(U[1],T[1],U[2],A); }
$$

$$
\text { true }
$$

If we define $W=U[2] T[2] S[2]$, namely,

$$
\begin{gathered}
>\mathrm{W}:=\operatorname{Mult}(\mathrm{U}[2], \mathrm{T}[2], \mathrm{S}[2], \mathrm{A}) ; \\
W:=\left[\begin{array}{cccccc}
0 & 0 & 0 & 1 & 0 & 0 \\
0 & 0 & 0 & 0 & 1 & 0 \\
0 & 0 & 0 & 0 & 0 & 1
\end{array}\right]
\end{gathered}
$$

then $\vartheta=\gamma \circ \beta \circ \alpha: L^{\prime} \longrightarrow L$ defined by $\vartheta(v(\mu))=\tau(\mu W)$ for all $\mu \in A^{1 \times 3}$ is an $A$ isomorphism:

$$
>\operatorname{TestIso}(\mathrm{U}[1], \mathrm{P}, \mathrm{W}, \mathrm{A}) \text {; }
$$

\section{true}

Performing algebraic simplification on $V[1]=U[1]^{T}$, namely, 


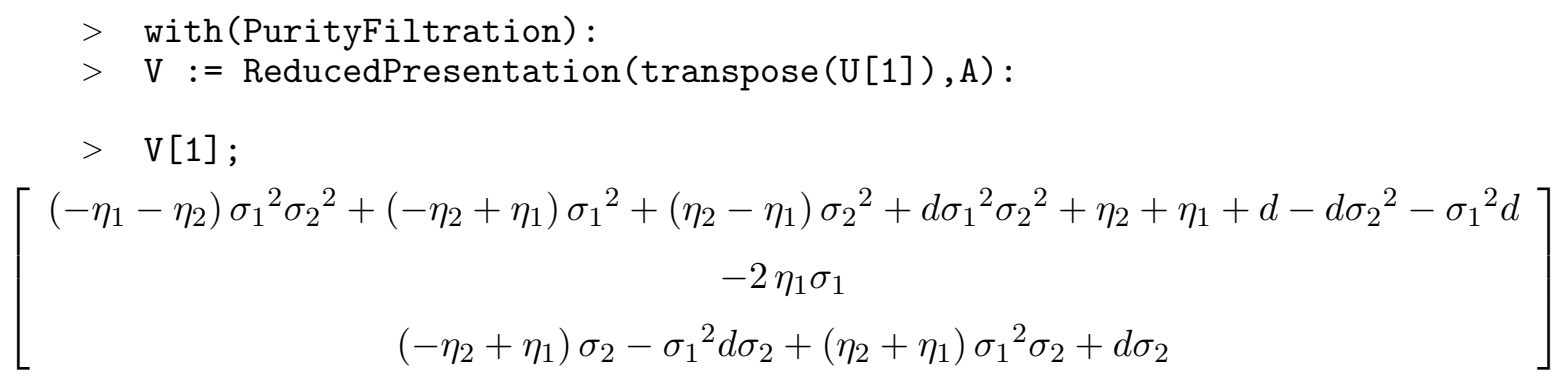

we get that $A /\left(A^{1 \times 3} V[1]\right) \cong A /\left(A^{1 \times 3} V[2]\right)$, where $V[2]$ is defined by

$>\mathrm{V}[2]$;

$$
\left[\begin{array}{c}
2 \eta_{1} \sigma_{1} \\
2 \eta_{1} \eta_{2} \sigma_{2} \\
2 \eta_{1} \eta_{2}+2 \eta_{1} d+2 \eta_{1}^{2}
\end{array}\right]
$$

the corresponding $A$-isomorphism being defined by the identity map, i.e.:

$>\mathrm{V}[3]$;

$$
[1]
$$

This result can be checked again:

$>$ TestIso(V[1],V[2],V[3],A);

true

If we denote by $Q$ a matrix satisfying $V[2]=Q^{T} V[1]$, namely,

$>\mathrm{Q}:=\operatorname{transpose}($ Factorize $(\mathrm{V}[2], \mathrm{V}[1], \mathrm{A}))$;

$$
Q:=\left[\begin{array}{ccc}
0 & \eta_{1} \sigma_{2} & 2 \eta_{1} \\
-1 & -\eta_{2} \sigma_{2} \sigma_{1} & \left(-\eta_{2}+\eta_{1}\right) \sigma_{1}-\sigma_{1} d \\
0 & -\eta_{1}+\eta_{1} \sigma_{2}^{2} & 2 \eta_{1} \sigma_{2}
\end{array}\right]
$$

then we have $V[2]^{T}=V[1]^{T} Q=U[1] Q$ and we get that $L^{\prime} \cong M=A^{1 \times 3} /\left(A V[2]^{T}\right)=$ $A^{1 \times 3} /(A R)$, where $R=V[2]^{T} /\left(2 \eta_{1}\right)$, namely,

$$
>\mathrm{R}:=\operatorname{simplify}(\operatorname{evalm}(\operatorname{transpose}(\mathrm{V}[2]) /(2 * \text { eta }[1]))) \text {; }
$$

$$
R:=\left[\begin{array}{lll}
\sigma_{1} & \eta_{2} \sigma_{2} & \eta_{2}+d+\eta_{1}
\end{array}\right]
$$

and the $A$-isomorphism $\omega: L^{\prime} \longrightarrow M$ is defined by $\omega(v(\mu))=\pi(v Q)$ for all $v \in A^{1 \times 3}$, where $\pi: A^{1 \times 3} \longrightarrow M$ is the canonical projection onto $M$. Let us check again that $\omega$ is an $A$-isomorphism:

$$
>\operatorname{TestIso}(\mathrm{U}[1], \mathrm{R}, \mathrm{Q}, \mathrm{A}) \text {; }
$$

$\operatorname{RR} n^{\circ} 8225$ 
true

Then, $\omega^{-1}: M \longrightarrow L^{\prime}$ is defined by $\omega^{-1}\left(\pi(\eta)=v(\eta X[1])\right.$ for all $\eta \in A^{1 \times 3}$, where $X[1]$ is the first entry of $X$ defined by:

$$
\begin{aligned}
> & X:=\operatorname{InverseMorphism}(\mathrm{U}[1], \mathrm{R}, \mathrm{Q}, \mathrm{A}) \\
X & \left.:=\left[\begin{array}{ccc}
1 / 2 \frac{\sigma_{1}\left(-d+d \sigma_{2}{ }^{2}-\eta_{2}-\eta_{1} \sigma_{2}{ }^{2}+\eta_{1}-\eta_{2} \sigma_{2}{ }^{2}\right)}{\eta_{1}} & -1 & -1 / 2 \frac{\sigma_{2} \sigma_{1}\left(-\eta_{2}-\eta_{1}+d\right)}{\eta_{1}} \\
\frac{\sigma_{2}}{\eta_{1}} & 0 & -\eta_{1}{ }^{-1} \\
-1 / 2 \frac{\sigma_{2}{ }^{2}-1}{\eta_{1}} & 0 & 1 / 2 \frac{\sigma_{2}}{\eta_{1}}
\end{array}\right],\left[1 / 2 \eta_{1}{ }^{-1}\right]\right]
\end{aligned}
$$

Now, if we define $Z=X[1] W$, namely,

$$
\begin{aligned}
>Z & :=\operatorname{Mult}(\mathrm{X}[1], \mathrm{W}, \mathrm{A}) ; \\
Z & :=\left[\begin{array}{cccccc}
0 & 0 & 0 & 1 / 2 \frac{\sigma_{1}\left(-d+d \sigma_{2}{ }^{2}-\eta_{2}-\eta_{1} \sigma_{2}{ }^{2}+\eta_{1}-\eta_{2} \sigma_{2}{ }^{2}\right)}{\eta_{1}} & -1 & -1 / 2 \frac{\sigma_{2} \sigma_{1}\left(-\eta_{2}-\eta_{1}+d\right)}{\eta_{1}} \\
0 & 0 & 0 & \frac{\sigma_{2}}{\eta_{1}} & 0 & -\eta_{1}{ }^{-1} \\
0 & 0 & 0 & -1 / 2 \frac{\sigma_{2}{ }^{2}-1}{\eta_{1}} & 0 & 1 / 2 \frac{\sigma_{2}}{\eta_{1}}
\end{array}\right]
\end{aligned}
$$

then $\varpi=\omega^{-1} \circ \vartheta: M \longrightarrow L$ is the $A$-isomorphism defined by $\varpi(\pi(\eta))=\tau(\eta Z)$ for all $\eta \in A^{1 \times 3}$

$>$ TestIso $(\mathrm{R}, \mathrm{P}, \mathrm{Z}, \mathrm{A})$;

true

which shows that the linear differential time-delay system defining the string model with an interior mass is equivalent to the single differential time-delay equation:

$$
\dot{z}_{3}(t)+\left(\eta_{1}+\eta_{2}\right) z_{3}(t)+z_{1}\left(t-h_{1}\right)+\eta_{2} z_{2}\left(t-h_{2}\right)=0 .
$$

Finally, $\varpi^{-1}: L \longrightarrow M$ is defined by $\varpi^{-1}(\tau(\lambda))=\pi(\lambda Y[1])$ for all $\lambda \in A^{1 \times 6}$, where $Y[1]$ is the first entry of $Y$ defined by

$$
\begin{aligned}
& >\mathrm{Y}:=\operatorname{Inverse} \operatorname{Morphism}(\mathrm{R}, \mathrm{P}, \mathrm{Z}, \mathrm{A}) \\
& {\left[\left[\begin{array}{ccc}
0 & -\eta_{2} \sigma_{2} & -\eta_{2}+\eta_{1}-d \\
0 & \eta_{2} \sigma_{2} & d+\eta_{1}+\eta_{2} \\
0 & -\eta_{1} \sigma_{2} & 0 \\
0 & \eta_{1} \sigma_{2} & 2 \eta_{1} \\
-1 & -\sigma_{2} \sigma_{1} \eta_{2} & -d \sigma_{1}-\sigma_{1} \eta_{2}+\eta_{1} \sigma_{1} \\
0 & \eta_{1} \sigma_{2}{ }^{2}-\eta_{1} & 2 \eta_{1} \sigma_{2}
\end{array}\right],\left[\begin{array}{l}
0 \\
0 \\
1 \\
0
\end{array}\right]\right]}
\end{aligned}
$$

$\operatorname{RR} n^{\circ} 8225$ 
a fact which can be checked again:

$>\operatorname{TestIso}(\mathrm{P}, \mathrm{R}, \mathrm{Y}[1], \mathrm{A})$;

true

\subsection{Computation of bases of finitely generated free modules}

Example 28. Let us consider the first Weyl algebra $A=A_{1}(\mathbb{Q})$

$>A:=$ DefineOreAlgebra $(\operatorname{diff}=[\mathrm{d}, \mathrm{t}]$, polynom $=[\mathrm{t}])$ :

and the left $A$-module $M=A^{1 \times 3} /(A R)$ finitely presented by the matrix $R$ defined by:

$>\mathrm{R}:=\operatorname{evalm}([[\mathrm{d}, \mathrm{t}, 0]])$;

$$
R:=\left[\begin{array}{lll}
d & t & 0
\end{array}\right]
$$

The rank of the left $A$-module $M$ is:

$>\operatorname{OreRank}(\mathrm{R}, \mathrm{A})$;

Since $R$ admits a right inverse $S$ defined by

$>\mathrm{S}:=$ RightInverse $(\mathrm{R}, \mathrm{A})$;

$$
S:=\left[\begin{array}{c}
t \\
-d \\
0
\end{array}\right]
$$

$M$ is a stably free left $A$-module of rank 2, i.e., a free left $A$-module of rank 2 by 4 of Theorem 1. Using $M \oplus A \cong A^{1 \times 3}$ and the Cancellation theorem (see Theorem 11), let us compute a basis of $M$. Following Section 6.2.1, let us first compute $X=\left(\begin{array}{ll}R^{T} & \left(I_{3}-S R\right)^{T}\end{array}\right)^{T}$

$>\quad X:=\operatorname{stackmatrix}(R, 1-\operatorname{Mult}(\mathrm{S}, \mathrm{R}, \mathrm{A}))$;

$$
X:=\left[\begin{array}{ccc}
d & t & 0 \\
-d t+1 & -t^{2} & 0 \\
d^{2} & 2+d t & 0 \\
0 & 0 & 1
\end{array}\right]
$$

which defines the left $A$-isomorphism $g: A \oplus M \longrightarrow A^{1 \times 3}$ (see Section 6.2.1). Moreover, we have $A \oplus M \cong L=A^{1 \times 4} /(A P)$, where $P=\left(\begin{array}{ll}0 & R\end{array}\right)$ is defined by:

$>P:=\operatorname{augment}(\operatorname{evalm}([[0]]), R)$;

$\mathrm{RR} \mathrm{n}^{\circ} 8225$ 


$$
P:=\left[\begin{array}{llll}
0 & d & t & 0
\end{array}\right]
$$

Similarly, $A^{1 \times 2} \cong A^{1 \times 2} /\left(A R^{\prime}\right)$, where $R^{\prime}$ is defined by

$>\operatorname{Rp}:=\operatorname{evalm}([0 \$ 2]])$;

$$
R p:=\left[\begin{array}{ll}
0 & 0
\end{array}\right]
$$

and $A^{1 \times 3} \cong L^{\prime}=A^{1 \times 3} /\left(A P^{\prime}\right)$, where $P^{\prime}=\left(\begin{array}{ll}0 & R^{\prime}\end{array}\right)$ is defined by:

$>\mathrm{Pp}:=\operatorname{augment}(\operatorname{evalm}([[0]]), \mathrm{Rp})$;

$$
P p:=\left[\begin{array}{lll}
0 & 0 & 0
\end{array}\right]
$$

Let us check again that $f: L \longrightarrow L^{\prime}$ defined by $\left.f\left(\begin{array}{ll}\theta & \lambda\end{array}\right)\right)=\left(\begin{array}{ll}\theta & \lambda\end{array}\right) X$ for all $\theta \in A$ and for all $\lambda \in A^{1 \times 3}$, where $\tau: A^{1 \times 4} \longrightarrow L$ is the canonical projection onto $L$, is a left $A$-isomorphism:

$>$ TestIso(P, Pp, X, A);

true

We can now apply Algorithm 6 to $P, P^{\prime}$, and $X$

$>\mathrm{Q}:=\operatorname{map}($ collect, Cancellation(Rp, $\mathrm{X}, \mathrm{A}$, "splithom"), $[\mathrm{d}, \mathrm{t}])$ :

to obtain a left $A$-isomorphism $h: M \longrightarrow A^{1 \times 2}$ defined by $h(\pi(\lambda))=\lambda Q$ for all $\lambda \in A^{1 \times 3}$, where $\pi: A^{1 \times 3} \longrightarrow M$ is the canonical projection onto $M$ and $Q$ is a $3 \times 2$ matrix

$>\operatorname{rowdim}(\mathrm{Q}) ; \operatorname{coldim}(\mathrm{Q})$;

\section{3,2}

whose first column is defined by

$>\operatorname{submatrix}(Q, 1 \ldots 3,1 \ldots 1)$;

$$
\left[\begin{array}{c}
\left(-t^{4}-t^{3}\right) d^{2}+\left(-3 t^{2}-4 t^{3}-t^{4}\right) d-3 t^{2}-2 t^{3} \\
\left(t^{2}+t^{3}\right) d^{3}+\left(t^{3}+6 t+8 t^{2}\right) d^{2}+\left(15 t+6 t^{2}+6\right) d+6+6 t \\
-d^{2} t^{3}+\left(-t^{3}-3 t^{2}\right) d-t-2 t^{2}
\end{array}\right]
$$

and whose second column is defined by:

$$
\begin{gathered}
>\operatorname{submatrix}(\mathrm{Q}, 1.3,2.2) ; \\
{\left[\begin{array}{c}
\left(-t^{4}-t^{2}-2 t^{3}\right) d^{3}+\left(-t-t^{4}-6 t^{2}-6 t^{3}\right) d^{2}+\left(-6 t^{2}-2 t+1-3 t^{3}\right) d-t^{2}+2 \\
2+\left(t+2 t^{2}+t^{3}\right) d^{4}+\left(3+10 t^{2}+12 t+t^{3}\right) d^{3}+\left(14+24 t+7 t^{2}\right) d^{2}+(10 t+12) d \\
\left(-t^{3}-t^{2}\right) d^{3}+\left(-t-5 t^{2}-t^{3}\right) d^{2}+\left(-3 t-3 t^{2}\right) d+1-t
\end{array}\right]}
\end{gathered}
$$

Let us check again that $h$ is a left $A$-isomorphism:

$$
>\operatorname{TestIso}(\mathrm{R}, \mathrm{Rp}, \mathrm{Q}, \mathrm{A}) \text {; }
$$

$\mathrm{RR} \mathrm{n}^{\circ} 8225$ 
true

Thus, the matrix $Q$ defines an injective parametrization of $M$, i.e., we have $\operatorname{ker}_{A}(. Q)=A R$

$>\operatorname{SyzygyModule}(\mathrm{Q}, \mathrm{A})$;

$$
\left[\begin{array}{lll}
d & t & 0
\end{array}\right]
$$

and $Q$ admits a left inverse $T \in A^{2 \times 3}$

$$
\begin{aligned}
& >\mathrm{T}:=\operatorname{map}(\operatorname{collect}, \operatorname{Left} \operatorname{Inverse}(\mathrm{Q}, \mathrm{A}),[\mathrm{d}, \mathrm{t}]): \\
& >\operatorname{rowdim}(\mathrm{T}) ; \operatorname{coldim}(\mathrm{T}) ;
\end{aligned}
$$

whose first two columns are defined by

$>\operatorname{submatrix}(\mathrm{T}, 1 \ldots 2,1 \ldots 2)$;

$$
\left[\begin{array}{cc}
-2 & \left(t^{2}+t^{3}\right) d^{2}+\left(5 t+7 t^{2}+t^{3}\right) d+4+10 t+5 t^{2} \\
t & -2 t^{2}-t^{3}-t^{3} d
\end{array}\right]
$$

and whose last column is defined by:

$>\quad \operatorname{submatrix}(\mathrm{T}, 1 \ldots 2,3 \ldots 3)$;

$$
\left[\begin{array}{c}
\left(t+2 t^{2}+t^{3}\right) d^{3}+\left(3+11 t+9 t^{2}+t^{3}\right) d^{2}+\left(11+19 t+6 t^{2}\right) d+7+6 t \\
\left(-t^{3}-t^{2}\right) d^{2}+\left(-t-4 t^{2}-t^{3}\right) d+1-2 t-2 t^{2}
\end{array}\right]
$$

Hence, we get the exact sequence $0 \longrightarrow A \stackrel{. R}{\longrightarrow} A^{1 \times 3} \stackrel{. Q}{\longrightarrow} A^{1 \times 2} \longrightarrow 0$, which shows that $M=\operatorname{coker}_{A}(. R) \cong \operatorname{im}_{A}(. Q)=A^{1 \times 3} Q=A^{1 \times 2}$. In particular, the residue classes of the rows of the left inverse $T$ of $Q$ define a basis of $M$, i.e., $\left\{\pi\left(T_{i} \bullet\right)\right\}_{i=1,2}$ is a basis of the free left $A$-module $M$ of rank 2.

Example 29. Let us consider the second Weyl algebra $A=A_{2}(\mathbb{Q})$

$$
\begin{aligned}
& >A:=\text { DefineOreAlgebra }(\operatorname{diff}=[\mathrm{d}[1], \mathrm{x}[1]], \operatorname{diff}=[\mathrm{d}[2], \mathrm{x}[2]], \\
& >\text { polynom }=[\mathrm{x}[1], \mathrm{x}[2]]):
\end{aligned}
$$

and the left $A$-module $M=A^{1 \times 3} /(A R)$ finitely presented by the matrix $R$ defined by:

$$
\begin{aligned}
>\mathrm{R}:=\operatorname{evalm}([[0, \mathrm{~d}[1], \mathrm{d}[2]+\mathrm{x}[1]]]) ; \\
R:=\left[\begin{array}{lll}
0 & d_{1} & d_{2}+x_{1}
\end{array}\right]
\end{aligned}
$$

The rank of left $A$-module $M$ is:

$>\operatorname{OreRank}(\mathrm{R}, \mathrm{A})$;

$\mathrm{RR} \mathrm{n}^{\circ} 8225$ 
Since $R$ admits a right inverse $S$ defined by

$>\mathrm{S}:=$ RightInverse $(\mathrm{R}, \mathrm{A})$;

$$
S:=\left[\begin{array}{c}
0 \\
d_{2}+x_{1} \\
-d_{1}
\end{array}\right]
$$

$M$ is a stably free left $A$-module of rank 2, i.e., a free left $A$-module of rank 2 by 4 of Theorem 1. Using $A \oplus M \cong A^{1 \times 3}$ and the Cancellation theorem (see Theorem 11), let us compute a basis of $M$. Following Section 6.2.1, let us first compute $X=\left(\begin{array}{ll}R^{T} & \left(I_{3}-S R\right)^{T}\end{array}\right)^{T}$

$>\quad X:=\operatorname{stackmatrix}(R, 1-\operatorname{Mult}(\mathrm{S}, \mathrm{R}, \mathrm{A}))$;

$$
X:=\left[\begin{array}{ccc}
0 & d_{1} & d_{2}+x_{1} \\
1 & 0 & 0 \\
0 & -\left(d_{2}+x_{1}\right) d_{1}+1 & -\left(d_{2}+x_{1}\right)^{2} \\
0 & d_{1}{ }^{2} & 2+d_{1} d_{2}+d_{1} x_{1}
\end{array}\right]
$$

which defines the left $A$-isomorphism $g: A \oplus M \longrightarrow A^{1 \times 3}$ (see Section 6.2.1). Moreover, we have $A \oplus M \cong L=A^{1 \times 4} /(A P)$, where $P=\left(\begin{array}{ll}0 & R\end{array}\right)$ is defined by:

$$
\begin{aligned}
>\mathrm{P}:=\operatorname{augment}(\operatorname{evalm}([0]]), \mathrm{R}) & ; \\
P & :=\left[\begin{array}{llll}
0 & 0 & d_{1} & d_{2}+x_{1}
\end{array}\right]
\end{aligned}
$$

Similarly, $A^{1 \times 2} \cong A^{1 \times 2} /\left(A R^{\prime}\right)$, where $R^{\prime}$ is defined by

$>\operatorname{Rp}:=\operatorname{evalm}([0 \$ 2]])$;

$$
R p:=\left[\begin{array}{ll}
0 & 0
\end{array}\right]
$$

and $A^{1 \times 3} \cong L^{\prime}=A^{1 \times 3} /\left(A P^{\prime}\right)$, where $P^{\prime}=\left(\begin{array}{ll}0 & R^{\prime}\end{array}\right)$ is defined by:

$>\mathrm{Pp}:=\operatorname{augment}(\operatorname{evalm}([[0]]), \mathrm{Rp})$;

$$
P p:=\left[\begin{array}{lll}
0 & 0 & 0
\end{array}\right]
$$

Let us check again that $f: L \longrightarrow L^{\prime}$ defined by $\left.f\left(\begin{array}{ll}\theta & \lambda\end{array}\right)\right)=\left(\begin{array}{ll}\theta & \lambda\end{array}\right) X$ for all $\theta \in A$ and for all $\lambda \in A^{1 \times 3}$, where $\tau: A^{1 \times 4} \longrightarrow L$ is the canonical projection onto $L$, is a left $A$-isomorphism:

$>$ TestIso(P, Pp, X, A);

true

We can apply Algorithm 6 to $P, P^{\prime}$, and $X$

$$
>\mathrm{Q}:=\text { Cancellation(Rp, } \mathrm{X}, \mathrm{A}, \text { "splithom"); }
$$




$$
Q:=\left[\begin{array}{cc}
d_{1} & d_{2}+x_{1} \\
-\left(d_{2}+x_{1}\right) d_{1}+1 & -\left(d_{2}+x_{1}\right)^{2} \\
d_{1}{ }^{2} & 2+d_{1} d_{2}+d_{1} x_{1}
\end{array}\right]
$$

to obtain a left $A$-isomorphism $h: M \longrightarrow A^{1 \times 2}$ defined by $h(\pi(\lambda))=\lambda Q$ for all $\lambda \in A^{1 \times 3}$, where $\pi: A^{1 \times 3} \longrightarrow M$ is the canonical projection onto $M$. Let us check again that $h$ is a left $A$-isomorphism:

$$
>\operatorname{TestIso}(\mathrm{R}, \mathrm{Rp}, \mathrm{Q}, \mathrm{A}) \text {; }
$$

true

Thus, the matrix $Q$ defines an injective parametrization of $M$, i.e., we have $\operatorname{ker}_{A}(. Q)=A R$

$>\operatorname{SyzygyModule}(\mathrm{Q}, \mathrm{A})$;

$$
\left[\begin{array}{lll}
0 & d_{1} & d_{2}+x_{1}
\end{array}\right]
$$

and $Q$ admits a left inverse $T \in A^{2 \times 3}$

$>\mathrm{T}:=$ LeftInverse $(\mathrm{Q}, \mathrm{A})$;

$$
T:=\left[\begin{array}{ccc}
d_{2}+x_{1} & 1 & 0 \\
-d_{1} & 0 & 1
\end{array}\right]
$$

Hence, we get the exact sequence $0 \longrightarrow A \stackrel{. R}{\longrightarrow} A^{1 \times 3} \stackrel{. Q}{\longrightarrow} A^{1 \times 2} \longrightarrow 0$, which shows that $M=\operatorname{coker}_{A}(. R) \cong \operatorname{im}_{A}(. Q)=A^{1 \times 3} Q=A^{1 \times 2}$. In particular, the residue classes of the rows of the left inverse $T$ of $Q$ define a basis of $M$, i.e., $\left\{\pi\left(T_{i} \bullet\right)\right\}_{i=1,2}$ is a basis of the free left $A$-module $M$ of rank 2 .

Let us compare this approach with the ones developed in $[11,36]$. Let us first check if we can compute a basis of $M$ by means of a minimal parametrization [11].

$>\mathrm{K}:=$ MinimalParametrizations $(\mathrm{R}, \mathrm{A})$;

$$
K:=\left[\left[\begin{array}{cc}
1 & 0 \\
0 & -d_{2}{ }^{2}-2 d_{2} x_{1}-x_{1}{ }^{2} \\
0 & 2+d_{1} d_{2}+d_{1} x_{1}
\end{array}\right],\left[\begin{array}{cc}
1 & 0 \\
0 & -d_{1} d_{2}-d_{1} x_{1}+1 \\
0 & d_{1}{ }^{2}
\end{array}\right]\right]
$$

We obtain that $\operatorname{ker}_{A}(. K[i])=A R$ for $i=1,2$, and thus, $M \cong A^{1 \times 3} K[i] \subseteq A^{1 \times 2}$. But none of the $K[i]$ 's admits a left inverse:

$>\operatorname{map}($ LeftInverse, $\mathrm{K}, \mathrm{A})$;

\section{[[], []]}

Therefore, we get $A^{1 \times 3} K[i] \subsetneq A^{1 \times 2}$, and thus these two minimal parametrizations do not define an injective parametrization of $M$.

$\mathrm{RR} \mathrm{n}^{\circ} 8225$ 
Let us now use the algorithm developed in [36] and implemented in the STAFFORD package ([36]) for the computation of bases of free left $A$-modules of rank greater than or equal to 2 :

$>\mathrm{Qp}:=$ InjectiveParametrization $(\mathrm{R}, \mathrm{A})$;

$Q p:=\left[\begin{array}{cc}d_{2}+x_{1} & d_{1} \\ d_{1} d_{2}{ }^{2}+2 d_{2} d_{1} x_{1}-d_{2}{ }^{2}-2 d_{2} x_{1}+d_{1} x_{1}{ }^{2}-x_{1}{ }^{2} & -d_{1}+d_{1}{ }^{2} d_{2}+d_{1}{ }^{2} x_{1}+1-d_{1} d_{2}-d_{1} x_{1} \\ 2-d_{1}{ }^{2} d_{2}+d_{1} d_{2}-d_{1}{ }^{2} x_{1}-2 d_{1}+d_{1} x_{1} & -d_{1}{ }^{3}+d_{1}{ }^{2}\end{array}\right]$

We obtain that $\operatorname{ker}_{A}\left(. Q^{\prime}\right)=A R$

$>\operatorname{SyzygyModule}(\mathrm{Qp}, \mathrm{A})$;

$$
\left[\begin{array}{lll}
0 & d_{1} & d_{2}+x_{1}
\end{array}\right]
$$

and $Q^{\prime}$ admits a left inverse $T^{\prime}$ defined by

$>\operatorname{Tp}:=\operatorname{LeftInverse}(\mathrm{Qp}, \mathrm{A})$;

$$
T p:=\left[\begin{array}{ccc}
d_{1}^{2}-d_{1} & 0 & 1 \\
-d_{1} d_{2}-d_{1} x_{1}+d_{2}+x_{1}+1 & 1 & 0
\end{array}\right]
$$

which yields $M \cong A^{1 \times 2} Q^{\prime}=A^{1 \times 2}$. Finally, $\left\{\pi\left(T_{i \bullet}^{\prime}\right)\right\}_{i=1,2}$ is also a basis of $M$.

Example 30. Let us consider the first Weyl algebra $A=A_{1}(\mathbb{Q})$

$$
>A:=\text { DefineOreAlgebra }(\operatorname{diff}=[\mathrm{d}, \mathrm{t}] \text {, polynom=[t]): }
$$

and the left $A$-module $M=A^{1 \times 4} /\left(A^{1 \times 2} R\right)$ finitely presented by the matrix $R$ defined by:

$>R:=\operatorname{evalm}([[0, \mathrm{~d}, 0,-1],[\mathrm{d}, 0,-\mathrm{t}, 0]])$;

$$
R:=\left[\begin{array}{cccc}
0 & d & 0 & -1 \\
d & 0 & -t & 0
\end{array}\right]
$$

The rank of the finitely generated left $A$-module $M$ is:

$>\operatorname{OreRank}(\mathrm{R}, \mathrm{A})$;

Since $R$ admits a right inverse $S$ defined by

$>\mathrm{S}:=$ RightInverse $(\mathrm{R}, \mathrm{A})$;

$\mathrm{RR} \mathrm{n}^{\circ} 8225$

$$
S:=\left[\begin{array}{cc}
0 & t \\
0 & 0 \\
0 & d \\
-1 & 0
\end{array}\right]
$$


$M$ is a stably free left $A$-module of rank 2, i.e., a free left $A$-module of rank 2 by 4 of Theorem 1. Using $A^{1 \times 2} \oplus M \cong A^{1 \times 4}$ and the Cancellation theorem (see Theorem 11), let us compute a basis of $M$. Following Section 6.2.1, let us first compute $X=\left(\begin{array}{ll}R^{T} & \left(I_{4}-S R\right)^{T}\end{array}\right)^{T}$

$>\quad X:=\operatorname{stackmatrix}(R, 1-\operatorname{Mult}(\mathrm{S}, \mathrm{R}, \mathrm{A}))$;

$$
X:=\left[\begin{array}{cccc}
0 & d & 0 & -1 \\
d & 0 & -t & 0 \\
-d t+1 & 0 & t^{2} & 0 \\
0 & 1 & 0 & 0 \\
-d^{2} & 0 & 2+d t & 0 \\
0 & d & 0 & 0
\end{array}\right]
$$

which defines the left $A$-isomorphism $g: A^{1 \times 2} \oplus M \longrightarrow A^{1 \times 4}$ (see Section 6.2.1). Moreover, we have $A^{1 \times 2} \oplus M \cong L=A^{1 \times 6} /\left(A^{1 \times 2} P\right)$, where $P=\left(\begin{array}{ll}0 & R\end{array}\right) \in A^{2 \times 6}$ is defined by:

$>P:=\operatorname{augment}(\operatorname{evalm}([[0,0],[0,0]]), R)$;

$$
P:=\left[\begin{array}{cccccc}
0 & 0 & 0 & d & 0 & -1 \\
0 & 0 & d & 0 & -t & 0
\end{array}\right]
$$

Similarly, $A^{1 \times 3} \cong A^{1 \times 2} /\left(A R^{\prime}\right)$, where $R^{\prime}$ is defined by

$>\operatorname{Rp}:=\operatorname{evalm}([0 \$ 3]])$;

$$
R p:=\left[\begin{array}{lll}
0 & 0 & 0
\end{array}\right]
$$

and $A^{1 \times 4} \cong L^{\prime}=A^{1 \times 4} /\left(A P^{\prime}\right)$, where $P^{\prime}=\left(\begin{array}{ll}0 & R^{\prime}\end{array}\right) \in A^{1 \times 4}$ is defined by:

$$
\begin{aligned}
>P \mathrm{P}:=\operatorname{augment}(\operatorname{evalm}([[0]]), \mathrm{Rp}) & ; \\
P p & :=\left[\begin{array}{llll}
0 & 0 & 0 & 0
\end{array}\right]
\end{aligned}
$$

Let us check again that $f: L \longrightarrow L^{\prime}$ defined by $\left.f\left(\begin{array}{ll}\tau & \lambda\end{array}\right)\right)=\left(\begin{array}{ll}\theta & \lambda\end{array}\right) X$ for all $\theta \in A^{1 \times 2}$ and for all $\lambda \in A^{1 \times 4}$, where $\tau: A^{1 \times 6} \longrightarrow L$ is the canonical projection onto $L$, is a left $A$-isomorphism:

\section{$>\operatorname{TestIso}(\mathrm{P}, \mathrm{Pp}, \mathrm{X}, \mathrm{A})$;}

true

Applying Theorem 6 to $f$, we obtain a left $A$-isomorphism $h_{1}: M_{1} \longrightarrow A^{1 \times 3}$ defined by $h_{1}\left(\pi_{1}(\mu)\right)=\mu Q_{1}$ for all $\mu \in A^{1 \times 5}$, where $M_{1}=A^{1 \times 5} /\left(A^{1 \times 2} P_{1}\right) \cong A \oplus M, \pi_{1}: A^{1 \times 5} \longrightarrow M_{1}$ is the canonical projection onto $M_{1}$, and the matrix $P_{1}=\left(\begin{array}{ll}0 & R\end{array}\right) \in A^{2 \times 5}$ is defined by

$>P 1:=\operatorname{augment}(\operatorname{evalm}([[0],[0]]), R)$; 


$$
P 1:=\left[\begin{array}{ccccc}
0 & 0 & d & 0 & -1 \\
0 & d & 0 & -t & 0
\end{array}\right]
$$

and $Q_{1} \in A^{5 \times 3}$ is defined by:

$>$ Q1 := Cancellation(Rp, X, A, "splithom");

$$
Q 1:=\left[\begin{array}{ccc}
d^{2} & -t & -d \\
-(d t-1) d & t^{2} & d t-1 \\
1 & 0 & 0 \\
-d^{3} & 2+d t & d^{2} \\
d & 0 & 0
\end{array}\right]
$$

Let us check again that $h_{1}$ is a left $A$-isomorphism:

$>$ TestIso(P1,Rp,Q1,A);

true

Let $R^{\prime \prime}=\left(\begin{array}{ll}0 & 0\end{array}\right) \in A^{1 \times 2}$, namely,

$>\operatorname{Rpp}:=\operatorname{evalm}([[0 \$ 2]])$;

$$
R p p:=\left[\begin{array}{ll}
0 & 0
\end{array}\right]
$$

and $L^{\prime \prime}=A^{1 \times 2} /\left(A R^{\prime \prime}\right) \cong A^{1 \times 2}$. Applying Theorem 6 to $h_{1}$, we obtain a left $A$-isomorphism $h_{2}: M \longrightarrow A^{1 \times 2}$ defined by $h_{2}(\pi(\lambda))=\lambda Q_{2}$ for all $\lambda \in A^{1 \times 4}$, where $\pi: A^{1 \times 4} \longrightarrow M$ is the canonical projection onto $M$ and $Q_{2}$ is the matrix defined by:

$>$ Q2 := Cancellation(Rpp,Q1,A,"splithom");

$$
Q 2:=\left[\begin{array}{cc}
t^{2}+t^{2} d^{2}+d t-1 & d t-1+(d t-1) d^{2} \\
-t & -d \\
2+d t+d^{3} t+3 d^{2} & d^{2}+d^{4} \\
-1-d t & -d^{2}
\end{array}\right]
$$

Thus, $Q_{2}$ is an injective parametrization of $M$, namely, $\operatorname{ker}_{A}\left(. Q_{2}\right)=A^{1 \times 2} R$,

$>$ SyzygyModule $(\mathrm{Q} 2, \mathrm{~A})$;

$$
\left[\begin{array}{cccc}
d & 0 & -t & 0 \\
0 & d & 0 & -1
\end{array}\right]
$$

or equivalently, $M \cong A^{1 \times 4} Q_{2}$, and $Q_{2}$ admits a left inverse $T_{2}$ defined by

$$
>\mathrm{T} 2:=\text { LeftInverse }(\mathrm{Q} 2, \mathrm{~A}) \text {; }
$$

RR n ${ }^{\circ} 8225$ 


$$
\left[\begin{array}{cccc}
0 & 0 & 1 & 1+d^{2} \\
-1 & -t & 0 & -d t+1
\end{array}\right]
$$

i.e., $A^{1 \times 4} Q_{2}=A^{1 \times 2}$, which shows again that $M \cong A^{1 \times 2}$, i.e., $M$ is a free left $A$-module of rank 2 , and the residue classes of the rows of $T_{2}$ define a basis of $M$.

\subsection{Cancellation theorem}

Example 31. Let us consider the third Weyl algebra $A=A_{3}(\mathbb{Q})$

$>A:=$ DefineOreAlgebra(diff $=[\mathrm{d} 1, \mathrm{x} 1]$, $\operatorname{diff}=[\mathrm{d} 2, \mathrm{x} 2]$, $\operatorname{diff}=[\mathrm{d} 3, \mathrm{x} 3]$, $>$ polynom $=[\mathrm{x} 1, \mathrm{x} 2, \mathrm{x} 3])$ :

and the left $A$-module $M=A^{1 \times 3} /(A R)$ finitely presented by the matrix $R$ defining the divergence operator in $\mathbb{R}^{3}$, namely:

$>\mathrm{R}:=\operatorname{evalm}([[\mathrm{d} 1, \mathrm{~d} 2, \mathrm{~d} 3]])$;

$$
R:=\left[\begin{array}{lll}
d 1 & d 2 & d 3
\end{array}\right]
$$

See Example 16. Moreover, let us consider $d=d 1$

$$
>\mathrm{d}:=\mathrm{d} 1
$$

and the element $m=\pi(\lambda) \in M$ defined by

$$
\begin{aligned}
&>\operatorname{lambda}:=\operatorname{evalm}([0,0,-\mathrm{x} 1]]) ; \\
& \lambda:=\left[\begin{array}{lll}
0 & 0 & -x 1
\end{array}\right]
\end{aligned}
$$

where $\pi: A^{1 \times 3} \longrightarrow M$ is the canonical projection onto $M$. Let $Q \in A^{3 \times k}$ be a matrix such that $\operatorname{ker}_{A}(R)=.Q A^{k}$, for instance:

$>\mathrm{Q}:=\operatorname{Involution}(\operatorname{SyzygyModule}(\operatorname{Involution}(\mathrm{R}, \mathrm{A}), \mathrm{A}), \mathrm{A})$;

$$
Q:=\left[\begin{array}{ccc}
d 3 & d 2 & 0 \\
0 & -d 1 & d 3 \\
-d 1 & 0 & -d 2
\end{array}\right]
$$

Thus, we have $\operatorname{hom}_{A}(M, A) \cong \operatorname{ker}_{A}(R)=.Q A^{3}$.

Let us check that $(d, m)$ is a unimodular element of $A \oplus M$, i.e., $d e+\lambda Q \xi=1$ for certain $e \in A$ and $\xi \in A^{3}$, or equivalently that $S=\left(\begin{array}{ll}d & \lambda Q\end{array}\right)$ admits a right inverse $\left(e \xi^{T}\right)^{T}$. We first have

$$
>\quad \mathrm{L}:=\operatorname{Mult}(\text { lambda, } \mathrm{Q}, \mathrm{A}) \text {; }
$$

$\mathrm{RR} \mathrm{n}^{\circ} 8225$ 


$$
L:=\left[\begin{array}{lllll}
x 1 & d 1 & 0 & x 1 & d 2
\end{array}\right]
$$

which yields that $S=\left(\begin{array}{ll}d & \lambda Q\end{array}\right)$ is defined by:

$$
\begin{aligned}
& >\mathrm{S}:=\operatorname{augment}(\operatorname{evalm}([[\mathrm{d}]]), \mathrm{L}) \text {; } \\
& S:=\left[\begin{array}{llllll}
d 1 & x 1 & d 1 & 0 & x 1 & d 2
\end{array}\right]
\end{aligned}
$$

The matrix $S$ admits a right inverse $T$ defined by:

$>\mathrm{T}:=$ RightInverse $(\mathrm{S}, \mathrm{A})$;

$$
T:=\left[\begin{array}{c}
x 1 \\
-1 \\
0 \\
0
\end{array}\right]
$$

Hence, we get that $e$ is defined by

$$
>\mathrm{e}:=\mathrm{T}[1,1]
$$

$$
x 1
$$

and $\xi$ is defined by:

$>$ xi := submatrix $(\mathrm{T}, 2 \ldots 4,1 \ldots 1)$;

$$
\xi:=\left[\begin{array}{c}
-1 \\
0 \\
0
\end{array}\right]
$$

Let us check that $\mu=Q \xi$, namely,

$>\mathrm{mu}:=\operatorname{Mult}(\mathrm{Q}, \mathrm{xi}, \mathrm{A})$;

$$
\mu:=\left[\begin{array}{c}
-d 3 \\
0 \\
d 1
\end{array}\right]
$$

is such that $\mu \in \operatorname{ker}_{A}(R$.), i.e., $\mu$ defines a left $A$-homomorphism from $M$ to $A$ :

$>\operatorname{Mult}(\mathrm{R}, \mathrm{mu}, \mathrm{A})$;

$$
[0]
$$

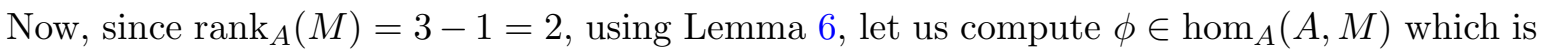
such that $\phi(d)+m$ is a unimodular element of $M$. 
$>\mathrm{U}:=$ ReductionOfUnimodularElement(R, d, lambda, e,mu, A, "splithom"):

The output of the command ReductionOfUnimodularElement contains two entries

$>\operatorname{nops}(\mathrm{U})$;

the first one $U[1]$, namely,

$>\mathrm{U}[1]$;

$$
\left[\begin{array}{lll}
0 & x 1+1 & 0
\end{array}\right]
$$

defines an element $\bar{m}=\pi(U[1]) \in M$ which is such that $\phi(a)=a \bar{m}$ for all $a \in A$. Moreover, the second entry $U[2]$ of $U$, namely,

$>\mathrm{U}[2]$;

$$
\left[\begin{array}{c}
-d 3+d 3 d 1^{2} x 1^{2}+d 3 d 1^{2} x 1-x 1 d 2+d 2 x 1^{2} d 1+2 d 3 d 1 x 1+2 d 1 d 3 \\
-x 1 d 1-x 1^{2} d 1^{2}+1 \\
-d 1-d 1^{3} x 1-x 1^{2} d 1^{3}-4 x 1 d 1^{2}-3 d 1^{2}
\end{array}\right]
$$

defines $\varphi \in \operatorname{hom}_{A}(M, A)$ satisfying:

$$
\varphi(\phi(d)+m)=\varphi(\pi(\lambda+d U[1]))=(\lambda+d U[1]) U[2]=1 .
$$

Finally, let us check again this result. We first have $u=\lambda+d U[1]$

$>\quad u:=\operatorname{simplify}(\operatorname{evalm}(\operatorname{lambda}+\operatorname{Mult}(\mathrm{d}, \mathrm{U}[1], \mathrm{A})))$;

$$
u:=\left[\begin{array}{lll}
0 & d 1+x 1 d 1+1 & -x 1
\end{array}\right]
$$

then we have $u U[2]=1$ since

$>\operatorname{Mult}(\mathrm{u}, \mathrm{U}[2], \mathrm{A})$;

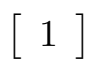

and $R U[2]=0$

$>\operatorname{Mult}(\mathrm{R}, \mathrm{U}[2], \mathrm{A})$;

$$
[0]
$$

which shows that $\varphi(\pi(\nu))=\nu U[2]$ for all $\nu \in A^{1 \times 3}$ defines a left $A$-homomorphism from $M$ to $A$, i.e., $\varphi \in \operatorname{hom}_{A}(M, A)$, which satisfies $\varphi(\phi(d)+m)=1$. 
Example 32. Let us consider the first Weyl algebra $A=A_{1}(\mathbb{Q})$

$>A:=$ DefineOreAlgebra $(\operatorname{diff}=[\mathrm{d}, \mathrm{t}]$, polynom $=[\mathrm{t}])$ :

the left $A$-module $M=A^{1 \times 2} /\left(A^{1 \times 2} R\right)$ finitely presented by the matrix $R$ defined by

$>R:=\operatorname{evalm}\left(\left[\left[\mathrm{t}^{\wedge} 2, \mathrm{t}\right],[\mathrm{t} * \mathrm{~d}+2, \mathrm{~d}]\right]\right)$;

$$
R:=\left[\begin{array}{cc}
t^{2} & t \\
t d+2 & d
\end{array}\right]
$$

and the left $A$-module $M^{\prime}=A /\left(A^{1 \times 2} R^{\prime}\right)$ finitely presented by the matrix $R^{\prime}$ defined by:

$>\operatorname{Rp}:=\operatorname{evalm}\left(\left[\left[\mathrm{t}^{\wedge} 2\right],[\mathrm{t} * \mathrm{~d}+2]\right]\right)$;

$$
R p:=\left[\begin{array}{c}
t^{2} \\
t d+2
\end{array}\right]
$$

Let us also consider the left $A$-homomorphism $\chi: M^{\prime} \longrightarrow M$ defined by $\chi\left(\pi^{\prime}(a)\right)=\pi(a W)$ for all $a \in A$, where $\pi: A \longrightarrow M$ (resp., $\pi: A^{1 \times 2} \longrightarrow M^{\prime}$ ) is the canonical projection onto $M$ (resp., $M^{\prime}$ ), and the matrix $W$ is defined by:

$>\mathrm{W}:=\operatorname{evalm}([[1,0]])$;

$$
W:=\left[\begin{array}{ll}
1 & 0
\end{array}\right]
$$

For more details, see Example 18. We can check that $\chi$ is a left $A$-isomorphism:

$>$ TestIso(Rp, R, W, A);

true

Since we have $M \cong M^{\prime}$, Theorem 12 and (60) then show that $A^{1 \times 4} \oplus \widetilde{N} \cong A^{1 \times 3} \oplus \widetilde{N^{\prime}}$, where $\widetilde{N}=A^{1 \times 2} /(A \widetilde{R})$ and $\widetilde{N^{\prime}}=A^{1 \times 2} /\left(A^{1 \times 2} \widetilde{R^{\prime}}\right)$, and $\widetilde{R}$ (resp., $\widetilde{R^{\prime}}$ ) is the formal adjoint of $R$ (resp., $R^{\prime}$ ). The corresponding left $A$-isomorphism can be computed by the command AuslanderEquivalence:

$>0:=$ AuslanderEquivalence $(\mathrm{Rp}, \mathrm{R}, \mathrm{W}, \mathrm{A}, \mathrm{opt})$ :

We obtain that the left $A$-module $L=A^{1 \times 7} /\left(A^{1 \times 3} P\right)$ finitely presented by the first entry $P=L[1]$ of $O$, i.e.,

$$
>\mathrm{P}:=0[1] \text {; }
$$

$$
P:=\left[\begin{array}{ccccccc}
0 & 0 & 0 & 0 & 1 & 0 & 0 \\
0 & 0 & 0 & 0 & 0 & t^{2} & 1-t d \\
0 & 0 & 0 & 0 & 0 & t & -d
\end{array}\right]
$$

$\mathrm{RR} \mathrm{n}^{\circ} 8225$ 
is isomorphic to $A^{1 \times 4} \oplus \tilde{N}$, and the left $A$-module $L^{\prime}=A^{1 \times 7} /\left(A^{1 \times 3} P^{\prime}\right)$ finitely presented by the second entry $P^{\prime}=O[2]$ of $O$, namely,

$>\operatorname{Pp}:=0[2]$

$$
P p:=\left[\begin{array}{ccccccc}
0 & 0 & 0 & t^{2} & 1-t d & 0 & 0 \\
0 & 0 & 0 & 0 & 0 & 1 & 0 \\
0 & 0 & 0 & 0 & 0 & 0 & 1
\end{array}\right]
$$

is isomorphic to $A^{1 \times 3} \oplus \widetilde{N^{\prime}}$. Moreover, if $X=O[3]$, namely,

$>\mathrm{X}:=0[3]$

$$
X:=\left[\begin{array}{ccccccc}
0 & -1 & 0 & 1 & 0 & 0 & 0 \\
0 & 0 & -1 & 0 & 1 & 0 & 0 \\
1 & -t^{2} & -1+t d & 0 & 0 & 1 & 0 \\
0 & -t & d & 0 & 0 & 0 & 1 \\
0 & 0 & 0 & t^{2} & 1-t d & -1 & 0 \\
0 & 0 & 0 & t d+2 & -d^{2} & 0 & -d \\
0 & 0 & 0 & t^{2} & 1-t d & 0 & -t
\end{array}\right]
$$

then the left $A$-homomorphism $f: L \longrightarrow L^{\prime}$ defined by $f(\tau(\zeta))=\tau^{\prime}(\zeta X)$ for all $\zeta \in A^{1 \times 7}$, where $\tau: A^{1 \times 7} \longrightarrow L$ (resp., $\tau^{\prime}: A^{1 \times 7} \longrightarrow L^{\prime}$ ) is a left $A$-isomorphism:

$$
>\operatorname{TestIso}(\mathrm{P}, \mathrm{Pp}, \mathrm{X}, \mathrm{A}) \text {; }
$$

true

The inverse $f^{-1}: L^{\prime} \longrightarrow L$ of $f$ is defined by $f^{-1}\left(\tau^{\prime}(\alpha)\right)=\tau\left(\alpha X^{\prime}\right)$ for all $\alpha \in A^{1 \times 7}$, where $X^{\prime}$ is defined by:

$$
>\mathrm{Xp}:=0[5] ;
$$


We can check again that the above left $A$-homomorphism is an isomorphism:

$>$ TestIso(Pp, P,Xp, A);

true

If $P=\left(\begin{array}{ll}0 & P_{2}\end{array}\right)$, where $P_{2}$ is defined by

$>\mathrm{P} 2:=\operatorname{submatrix}(\mathrm{P}, 1 \ldots 3,2 \ldots 7)$;

$$
P 2:=\left[\begin{array}{cccccc}
0 & 0 & 0 & 1 & 0 & 0 \\
0 & 0 & 0 & 0 & t^{2} & 1-t d \\
0 & 0 & 0 & 0 & t & -d
\end{array}\right]
$$

and $P^{\prime}=\left(\begin{array}{ll}0 & P_{2}^{\prime}\end{array}\right)$, where $P_{2}^{\prime}$ is defined by

$>\mathrm{P} 2 \mathrm{p}:=\operatorname{submatrix}(\mathrm{Pp}, 1 \ldots 3,2.7)$;

$$
P 2 p:=\left[\begin{array}{cccccc}
0 & 0 & t^{2} & 1-t d & 0 & 0 \\
0 & 0 & 0 & 0 & 1 & 0 \\
0 & 0 & 0 & 0 & 0 & 1
\end{array}\right]
$$

$L_{2}=A^{1 \times 6} /\left(A^{1 \times 3} P_{2}\right)$, and $L_{2}^{\prime}=A^{1 \times 6} /\left(A^{1 \times 3} P_{2}^{\prime}\right)$, then Corollary 4 shows that $L_{2} \cong L_{2}^{\prime}$. Let us compute the corresponding left $A$-isomorphism $f_{2}$.

$>\mathrm{Y}:=$ Cancellation $(\mathrm{P} 2 \mathrm{p}, \mathrm{X}, \mathrm{A})$;

$$
Y:=\left[\begin{array}{cccccc}
0 & -1 & 0 & 1 & 0 & 0 \\
-1 & -1+t d & 1-t^{2} & 0 & 1 & 0 \\
0 & d & -t & 0 & 0 & 1 \\
0 & 0 & t^{2} & 1-t d & -1 & 0 \\
0 & 0 & t d+2 & -d^{2} & 0 & -d \\
0 & 0 & t^{2} & 1-t d & 0 & -t
\end{array}\right]
$$

We obtain that $f_{2}: L_{2} \longrightarrow L_{2}^{\prime}$ defined by $f_{2}\left(\tau_{2}(\xi)\right)=\tau_{2}^{\prime}(\xi Y)$ for all $\xi \in A^{1 \times 6}$ is a left $A$-isomorphism, where $\tau_{2}: A^{1 \times 6} \longrightarrow L_{2}$ (resp., $\tau_{2}^{\prime}: A^{1 \times 6} \longrightarrow L_{2}^{\prime}$ ) is the canonical projection onto $L_{2}$ (resp., $\left.L_{2}^{\prime}\right)$.

$>$ TestIso(P2,P2p, Y,A);

true

If $P_{2}=\left(\begin{array}{ll}0 & P_{3}\end{array}\right)$ and $P_{2}^{\prime}=\left(\begin{array}{ll}0 & P_{3}^{\prime}\end{array}\right)$, where

$>\mathrm{P} 3:=\operatorname{submatrix}(\mathrm{P} 2,1 \ldots 3,2.6)$;

$\mathrm{RR} \mathrm{n}^{\circ} 8225$ 


$$
\begin{aligned}
& P 3:=\left[\begin{array}{ccccc}
0 & 0 & 1 & 0 & 0 \\
0 & 0 & 0 & t^{2} & 1-t d \\
0 & 0 & 0 & t & -d
\end{array}\right] \\
& >\text { P3p }:=\text { submatrix }(\mathrm{P} 2 \mathrm{p}, 1 . .3,2.6) \text {; } \\
& P 3 p:=\left[\begin{array}{ccccc}
0 & t^{2} & 1-t d & 0 & 0 \\
0 & 0 & 0 & 1 & 0 \\
0 & 0 & 0 & 0 & 1
\end{array}\right]
\end{aligned}
$$

then applying again Corollary 4 to $L_{2} \cong L_{2}^{\prime}$, we get $L_{3} \cong L_{3}^{\prime}$, where $L_{3}=A^{1 \times 5} /\left(A^{1 \times 3} P_{3}\right)$ and $L_{3}^{\prime}=A^{1 \times 5} /\left(A^{1 \times 3} P_{3}^{\prime}\right)$. Let us compute the corresponding left $A$-isomorphism $f_{3}$.

$>\mathrm{Z}:=$ Cancellation $(\mathrm{P} 3 \mathrm{p}, \mathrm{Y}, \mathrm{A})$;

$$
Z:=\left[\begin{array}{ccccc}
1 & 1-t^{2} & -2+t d & 1 & 0 \\
0 & -t & d & 0 & 1 \\
0 & t^{2} & 1-t d & -1 & 0 \\
0 & t d+2 & -d^{2} & 0 & -d \\
0 & t^{2} & 1-t d & 0 & -t
\end{array}\right]
$$

We obtain that $f_{3}: L_{3} \longrightarrow L_{3}^{\prime}$ defined by $f_{3}\left(\tau_{3}(\nu)\right)=\tau_{3}^{\prime}(\nu Z)$ for all $\nu \in A^{1 \times 5}$ is an isomorphism, where $\tau_{3}: A^{1 \times 5} \longrightarrow L_{3}$ (resp., $\tau_{3}^{\prime}: A^{1 \times 5} \longrightarrow L_{3}^{\prime}$ ) is the canonical projection onto $L_{3}$ (resp., $L_{3}^{\prime}$ ). Let us check that $f_{3}$ is a left $A$-isomorphism.

$>$ TestIso(P3,P3p, Z, A);

true

Finally, if $P_{3}=\left(\begin{array}{ll}0 & P_{4}\end{array}\right)$, where

$>\mathrm{P} 4:=$ submatrix $(\mathrm{P} 3 \mathrm{p}, 1 \ldots 3,2 . .5)$;

$$
P 4:=\left[\begin{array}{cccc}
0 & 1 & 0 & 0 \\
0 & 0 & t^{2} & 1-t d \\
0 & 0 & t & -d
\end{array}\right]
$$

$P_{3}^{\prime}=\left(\begin{array}{ll}0 & P_{4}^{\prime}\end{array}\right)$, where

$>\mathrm{P} 4 \mathrm{p}:=$ submatrix $(\mathrm{P} 3,1 \ldots 3,2 . .5)$;

$$
P 4 p:=\left[\begin{array}{cccc}
t^{2} & 1-t d & 0 & 0 \\
0 & 0 & 1 & 0 \\
0 & 0 & 0 & 1
\end{array}\right]
$$

$\mathrm{RR} \mathrm{n}^{\circ} 8225$ 
$L_{4}=A^{1 \times 4} /\left(A^{1 \times 3} P_{4}\right)$, and $L_{4}^{\prime}=A^{1 \times 4} /\left(A^{1 \times 3} P_{4}^{\prime}\right)$, then we cannot deduce a left $A$-isomorphism

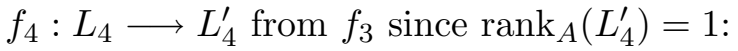

$>\mathrm{U}:=$ Cancellation $(\mathrm{P} 4 \mathrm{p}, \mathrm{Z}, \mathrm{A})$;

Error, (in Stafford/ReductionOfUnimodularElement) expecting that the rank of the

left module presented by the first matrix is at least 2.

Example 33. Let us consider the second Weyl algebra $A=A_{2}(\mathbb{Q})$

$>A:=\operatorname{DefineOreAlgebra}(\operatorname{diff}=[\mathrm{dx}, \mathrm{x}]$, diff $=[\mathrm{dy}, \mathrm{y}]$, polynom $=[\mathrm{x}, \mathrm{y}])$ :

the left $A$-module $M=A^{1 \times 3} /\left(A^{1 \times 6} R\right)$ finitely presented by the matrix $R$ defined by

$>\mathrm{R}:=\operatorname{transpose}(\operatorname{evalm}([[\mathrm{dx}, \mathrm{dy}, 0,0,0,0],[0,1,-1,0, \mathrm{dx}, \mathrm{dy}],[0,0, \mathrm{dx}, \mathrm{dy}, 0,0]]))$;

$$
R:=\left[\begin{array}{ccc}
d x & 0 & 0 \\
d y & 1 & 0 \\
0 & -1 & d x \\
0 & 0 & d y \\
0 & d x & 0 \\
0 & d y & 0
\end{array}\right]
$$

and the left $A$-module $M^{\prime}=A^{1 \times 2} /\left(A^{1 \times 3} R^{\prime}\right)$ finitely presented by $R^{\prime}$ defined by:

$>\operatorname{Rp}:=\operatorname{evalm}([[\mathrm{dx}, 0],[\mathrm{dy} / 2, \mathrm{dx} / 2],[0, \mathrm{dy}]])$;

$$
R p:=\left[\begin{array}{cc}
d x & 0 \\
1 / 2 d y & 1 / 2 d x \\
0 & d y
\end{array}\right]
$$

Let $\pi: A^{1 \times 3} \longrightarrow M$ (resp., $\pi^{\prime}: A^{1 \times 2} \longrightarrow M^{\prime}$ ) be the canonial projection onto $M$ (resp., $M^{\prime}$ ) and $f \in \operatorname{hom}_{A}\left(M^{\prime}, M\right)$ defined by $f\left(\pi^{\prime}(\lambda)\right)=\pi(\lambda S)$ for all $\lambda \in A^{1 \times 2}$, where $S$ is given by:

$>\mathrm{S}:=\operatorname{evalm}([[1,0,0],[0,0,1]])$;

$$
S:=\left[\begin{array}{lll}
1 & 0 & 0 \\
0 & 0 & 1
\end{array}\right]
$$

We can check that $f$ is a left $A$-isomorphism:

$>$ TestIso(Rp, R, S, A) ;

true

$\mathrm{RR} \mathrm{n}^{\circ} 8225$ 
Theorem 12 and (60) show that $A^{1 \times 6} \oplus \widetilde{N} \cong A^{1 \times 8} \oplus \widetilde{N^{\prime}}$, where $\widetilde{N}=A^{1 \times 3} /\left(A^{1 \times 2} \widetilde{R}\right)$ (resp. $\left.\widetilde{N^{\prime}}=A^{1 \times 6} /\left(A^{1 \times 3} \widetilde{R^{\prime}}\right)\right)$ is the left $A$-module finitely presented by the formal adjoint $\widetilde{R}$ (resp., $\widetilde{R^{\prime}}$ ) of $R$ (resp., $R^{\prime}$ ), namely:

$>\operatorname{Involution}(\mathrm{R}, \mathrm{A})$;

$$
\begin{aligned}
&>\text { Involution(Rp, } \mathrm{A}) ; {\left[\begin{array}{ccccc}
-d x & -1 / 2 d y & 0 \\
0 & -1 / 2 d x & -d y
\end{array}\right] } \\
& {\left[\begin{array}{ccccccc}
-d x & -d y & 0 & 0 & 0 & 0 \\
0 & 1 & -1 & 0 & -d x & -d y \\
0 & 0 & -d x & -d y & 0 & 0
\end{array}\right] }
\end{aligned}
$$

The corresponding isomorphism can be computed by the command AuslanderEquivalence:

$>0:=$ AuslanderEquivalence $(\mathrm{Rp}, \mathrm{R}, \mathrm{S}, \mathrm{A}, \mathrm{opt})$ :

The left $A$-module $L=A^{1 \times 14} /\left(A^{1 \times 5} P\right)$ finitely presented by the first entry $P=O[1]$ of $O$

$>P:=0[1]$

$$
P:=\left[\begin{array}{cccccccccccccc}
0 & 0 & 0 & 0 & 0 & 0 & 1 & 0 & 0 & 0 & 0 & 0 & 0 & 0 \\
0 & 0 & 0 & 0 & 0 & 0 & 0 & 1 & 0 & 0 & 0 & 0 & 0 & 0 \\
0 & 0 & 0 & 0 & 0 & 0 & 0 & 0 & -d x & -d y & 0 & 0 & 0 & 0 \\
0 & 0 & 0 & 0 & 0 & 0 & 0 & 0 & 0 & 1 & -1 & 0 & -d x & -d y \\
0 & 0 & 0 & 0 & 0 & 0 & 0 & 0 & 0 & 0 & -d x & -d y & 0 & 0
\end{array}\right]
$$

is isomorphic to $A^{1 \times 6} \oplus \tilde{N}$, and the left $A$-module $L^{\prime}=A^{1 \times 14} /\left(A^{1 \times 5} P^{\prime}\right)$ finitely presented by the second entry $P^{\prime}=O[2]$ of $O$, namely,

$$
\begin{gathered}
>P p:=0[2] ; \\
P p:=\left[\begin{array}{cccccccccccccc}
0 & 0 & 0 & 0 & 0 & 0 & 0 & 0 & -d x & -1 / 2 d y & 0 & 0 & 0 & 0 \\
0 & 0 & 0 & 0 & 0 & 0 & 0 & 0 & 0 & -1 / 2 d x & -d y & 0 & 0 & 0 \\
0 & 0 & 0 & 0 & 0 & 0 & 0 & 0 & 0 & 0 & 0 & 1 & 0 & 0 \\
0 & 0 & 0 & 0 & 0 & 0 & 0 & 0 & 0 & 0 & 0 & 0 & 1 & 0 \\
0 & 0 & 0 & 0 & 0 & 0 & 0 & 0 & 0 & 0 & 0 & 0 & 0 & 1
\end{array}\right]
\end{gathered}
$$

is isomorphic to $A^{1 \times 8} \oplus \widetilde{N^{\prime}}$.

The rank of the left $A$-module $L$ is:

$\mathrm{RR} \mathrm{n}^{\circ} 8225$ 


\section{$>\operatorname{OreRank}(\mathrm{P}, \mathrm{A})$;}

The rank of the left $A$-module $L^{\prime}$ is:

$>\operatorname{OreRank}(\mathrm{Pp}, \mathrm{A})$;

The left $A$-homomorphism $f_{1}: L \longrightarrow L^{\prime}$ defined by $f_{1}(\tau(\zeta))=\tau^{\prime}(\zeta O[3])$ for all $\zeta \in A^{1 \times 14}$, where $\tau: A^{1 \times 14} \longrightarrow L\left(\right.$ resp., $\left.\tau^{\prime}: A^{1 \times 14} \longrightarrow L^{\prime}\right)$ and the matrix $O[3]$ is defined by

$$
\begin{aligned}
& \text { O [3] ; } \\
& {\left[\begin{array}{cccccccccccccc}
0 & 0 & -1 & 0 & 0 & 0 & -d y & 0 & 1 & 0 & 0 & 0 & 0 & 0 \\
0 & 0 & 0 & 0 & -2 & 0 & 0 & -2 d y & 0 & 1 & 0 & 0 & 0 & 0 \\
0 & 0 & 0 & 0 & 0 & -1 & 0 & d x & 0 & 0 & 1 & 0 & 0 & 0 \\
1 & 0 & d x & d y & 0 & 0 & 0 & 0 & 0 & 0 & 0 & 1 & 0 & 0 \\
0 & 0 & 0 & -1 & 1 & 0 & d x & d y & 0 & 0 & 0 & 0 & 1 & 0 \\
0 & 1 & 0 & 0 & d x & d y & 0 & 0 & 0 & 0 & 0 & 0 & 0 & 1 \\
0 & 0 & 0 & 0 & 0 & 0 & 0 & 0 & -d x & -1 / 2 d y & 0 & -1 & -d y & 0 \\
0 & 0 & 0 & 0 & 0 & 0 & 0 & 0 & 0 & -1 / 2 d x & -d y & 0 & 0 & -1 \\
0 & 0 & 0 & 0 & 0 & 0 & -d y & 0 & 1 & 0 & 0 & 0 & 0 & 0 \\
0 & 0 & 0 & 0 & 0 & 0 & d x & 0 & 0 & 1 / 2 & 0 & 0 & 1 & 0 \\
0 & 0 & 0 & 0 & 0 & 0 & 0 & -d y & 0 & 1 / 2 & 0 & 0 & 0 & 0 \\
0 & 0 & 0 & 0 & 0 & 0 & 0 & d x & 0 & 0 & 1 & 0 & 0 & 0 \\
0 & 0 & 0 & 0 & 0 & 0 & 1 & 0 & 0 & 0 & 0 & 0 & 0 & 0 \\
0 & 0 & 0 & 0 & 0 & 0 & 0 & 1 & 0 & 0 & 0 & 0 & 0 & 0
\end{array}\right]}
\end{aligned}
$$

is a left $A$-isomorphism:

$>\operatorname{TestIso}(\mathrm{P}, \mathrm{Pp}, \mathrm{O}[3], \mathrm{A})$;

true

If $P=\left(\begin{array}{ll}0 & P_{2}\end{array}\right)$, where $P_{2}$ is defined by

$>\mathrm{P} 2:=\operatorname{submatrix}(\mathrm{P}, 1 \ldots 5,2 \ldots 14)$; 


$$
P 2:=\left[\begin{array}{ccccccccccccc}
0 & 0 & 0 & 0 & 0 & 1 & 0 & 0 & 0 & 0 & 0 & 0 & 0 \\
0 & 0 & 0 & 0 & 0 & 0 & 1 & 0 & 0 & 0 & 0 & 0 & 0 \\
0 & 0 & 0 & 0 & 0 & 0 & 0 & -d x & -d y & 0 & 0 & 0 & 0 \\
0 & 0 & 0 & 0 & 0 & 0 & 0 & 0 & 1 & -1 & 0 & -d x & -d y \\
0 & 0 & 0 & 0 & 0 & 0 & 0 & 0 & 0 & -d x & -d y & 0 & 0
\end{array}\right]
$$

then $L \cong A \oplus L_{2}$, where $L_{2}=A^{1 \times 13} /\left(A^{1 \times 5} P_{2}\right)$. The rank of $L_{2}$ is:

$>\operatorname{OreRank}(\mathrm{P} 2, \mathrm{~A})$;

Similarly, if $P^{\prime}=\left(\begin{array}{ll}0 & P_{2}^{\prime}\end{array}\right)$, where $P_{2}^{\prime}$ is defined by

$>\mathrm{P} 2 \mathrm{p}:=$ submatrix $(\mathrm{Pp}, 1 \ldots 5,2 \ldots 14)$

$$
P 2 p:=\left[\begin{array}{ccccccccccccc}
0 & 0 & 0 & 0 & 0 & 0 & 0 & -d x & -1 / 2 d y & 0 & 0 & 0 & 0 \\
0 & 0 & 0 & 0 & 0 & 0 & 0 & 0 & -1 / 2 d x & -d y & 0 & 0 & 0 \\
0 & 0 & 0 & 0 & 0 & 0 & 0 & 0 & 0 & 0 & 1 & 0 & 0 \\
0 & 0 & 0 & 0 & 0 & 0 & 0 & 0 & 0 & 0 & 0 & 1 & 0 \\
0 & 0 & 0 & 0 & 0 & 0 & 0 & 0 & 0 & 0 & 0 & 0 & 1
\end{array}\right]
$$

then $L^{\prime} \cong A \oplus L_{2}^{\prime}$, where $L_{2}^{\prime}=A^{1 \times 13} /\left(A^{1 \times 5} P_{2}^{\prime}\right)$. The rank of $L_{2}^{\prime}$ is:

$>\operatorname{OreRank}(\mathrm{P} 2 \mathrm{p}, \mathrm{A})$;

Corollary 4 then shows that $L_{2} \cong L_{2}^{\prime}$. Let us compute the corresponding left $A$-isomorphism $f_{2} \in \operatorname{hom}_{A}\left(L_{2}, L_{2}^{\prime}\right)$.

$>\mathrm{X}:=$ Cancellation $(\mathrm{P} 2 \mathrm{p}, \mathrm{O}[3], \mathrm{A})$; 


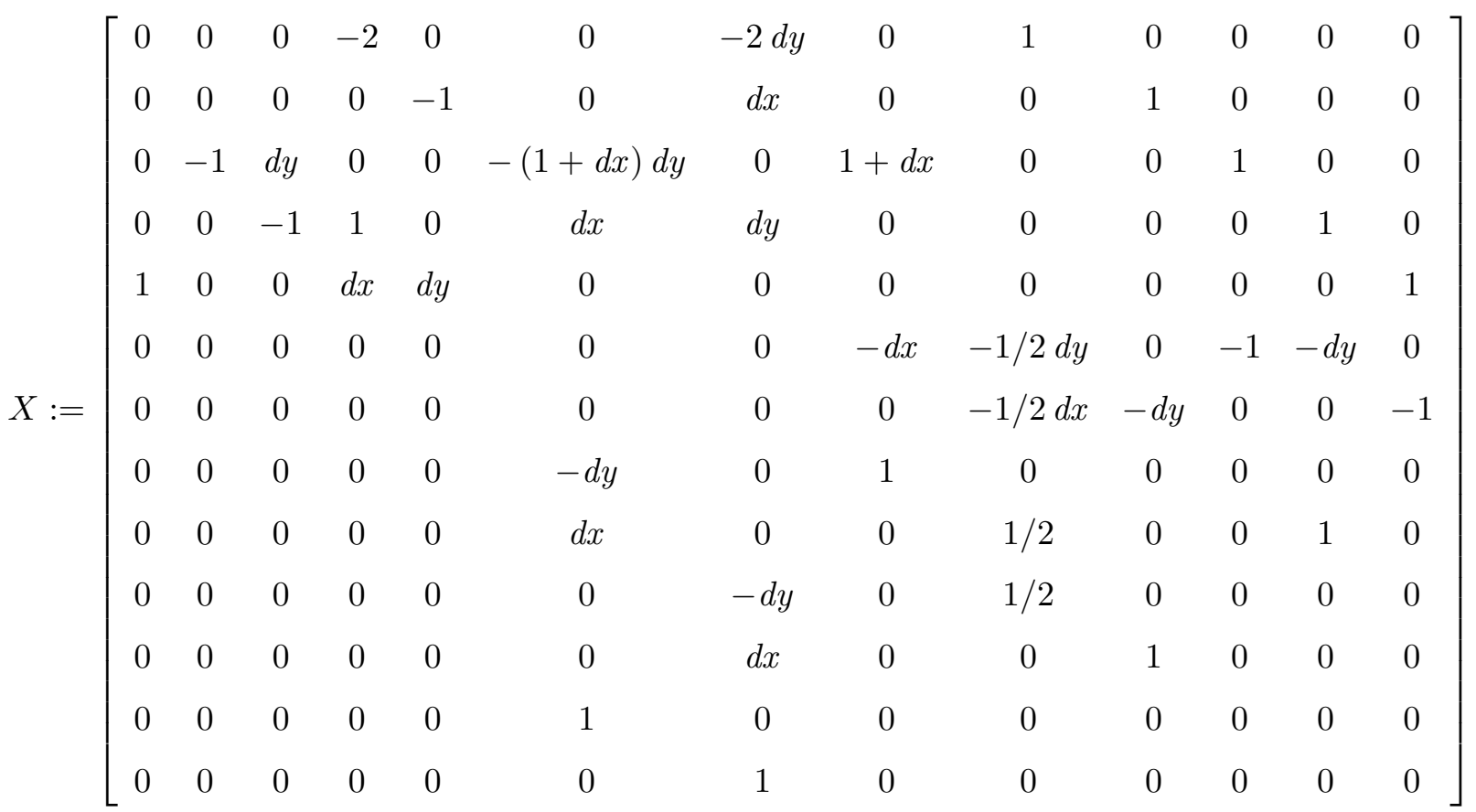

We obtain that $f_{2}: L_{2} \longrightarrow L_{2}^{\prime}$ defined by $f_{2}\left(\tau_{2}(\xi)\right)=\tau_{2}^{\prime}(\xi X)$ for all $\xi \in A^{1 \times 13}$ is left $A$ isomorphism, where $\tau_{2}: A^{1 \times 13} \longrightarrow L_{2}$ (resp., $\tau_{2}^{\prime}: A^{1 \times 13} \longrightarrow L_{2}^{\prime}$ ) is the canonical projection onto $L_{2}$ (resp., $L_{2}^{\prime}$ ). We can check again that $f_{2}$ is a left $A$-isomorphism:

$>$ TestIso(P2,P2p, X, A);

true

If $P_{2}=\left(\begin{array}{ll}0 & P_{3}\end{array}\right)$, where $P_{3}$ is defined by

$>\mathrm{P} 3:=$ submatrix $(\mathrm{P} 2,1 \ldots 5,2 \ldots 13)$;

$$
P 3:=\left[\begin{array}{cccccccccccc}
0 & 0 & 0 & 0 & 1 & 0 & 0 & 0 & 0 & 0 & 0 & 0 \\
0 & 0 & 0 & 0 & 0 & 1 & 0 & 0 & 0 & 0 & 0 & 0 \\
0 & 0 & 0 & 0 & 0 & 0 & -d x & -d y & 0 & 0 & 0 & 0 \\
0 & 0 & 0 & 0 & 0 & 0 & 0 & 1 & -1 & 0 & -d x & -d y \\
0 & 0 & 0 & 0 & 0 & 0 & 0 & 0 & -d x & -d y & 0 & 0
\end{array}\right]
$$

then $L_{2} \cong A \oplus L_{3}$, where $L_{3}=A^{1 \times 12} /\left(A^{1 \times 5} P_{3}\right)$. The rank of $L_{3}$ is:

$$
>\operatorname{OreRank}(\mathrm{P} 3, \mathrm{~A}) \text {; }
$$

If $P_{2}^{\prime}=\left(\begin{array}{ll}0 & P_{3}^{\prime}\end{array}\right)$, where $P_{3}^{\prime}$ is defined by

$\mathrm{RR} \mathrm{n}^{\circ} 8225$ 


$>$ P3p : $=$ submatrix $(\mathrm{P} 2 \mathrm{p}, 1 \ldots 5,2 \ldots 13)$
\[ 3 p:=\left[\begin{array}{cccccccccccc}0 & 0 & 0 & 0 & 0 & 0 & -d x & -1 / 2 d y & 0 & 0 & 0 & 0 \\ 0 & 0 & 0 & 0 & 0 & 0 & 0 & -1 / 2 d x & -d y & 0 & 0 & 0 \\ 0 & 0 & 0 & 0 & 0 & 0 & 0 & 0 & 0 & 1 & 0 & 0 \\ 0 & 0 & 0 & 0 & 0 & 0 & 0 & 0 & 0 & 0 & 1 & 0 \\ 0 & 0 & 0 & 0 & 0 & 0 & 0 & 0 & 0 & 0 & 0 & 1\end{array}\right] \]

then $L_{2}^{\prime} \cong A \oplus L_{3}^{\prime}$, where $L_{3}^{\prime}=A^{1 \times 12} /\left(A^{1 \times 5} P_{3}^{\prime}\right)$. The rank of $L_{3}^{\prime}$ is:

$>\operatorname{OreRank}(\mathrm{P} 3 \mathrm{p}, \mathrm{A})$;

\section{7}

Corollary 4 then shows that $L_{3} \cong L_{3}^{\prime}$. Let us compute the corresponding left $A$-isomorphism $f_{3} \in \operatorname{hom}_{A}\left(L_{3}, L_{3}^{\prime}\right)$.

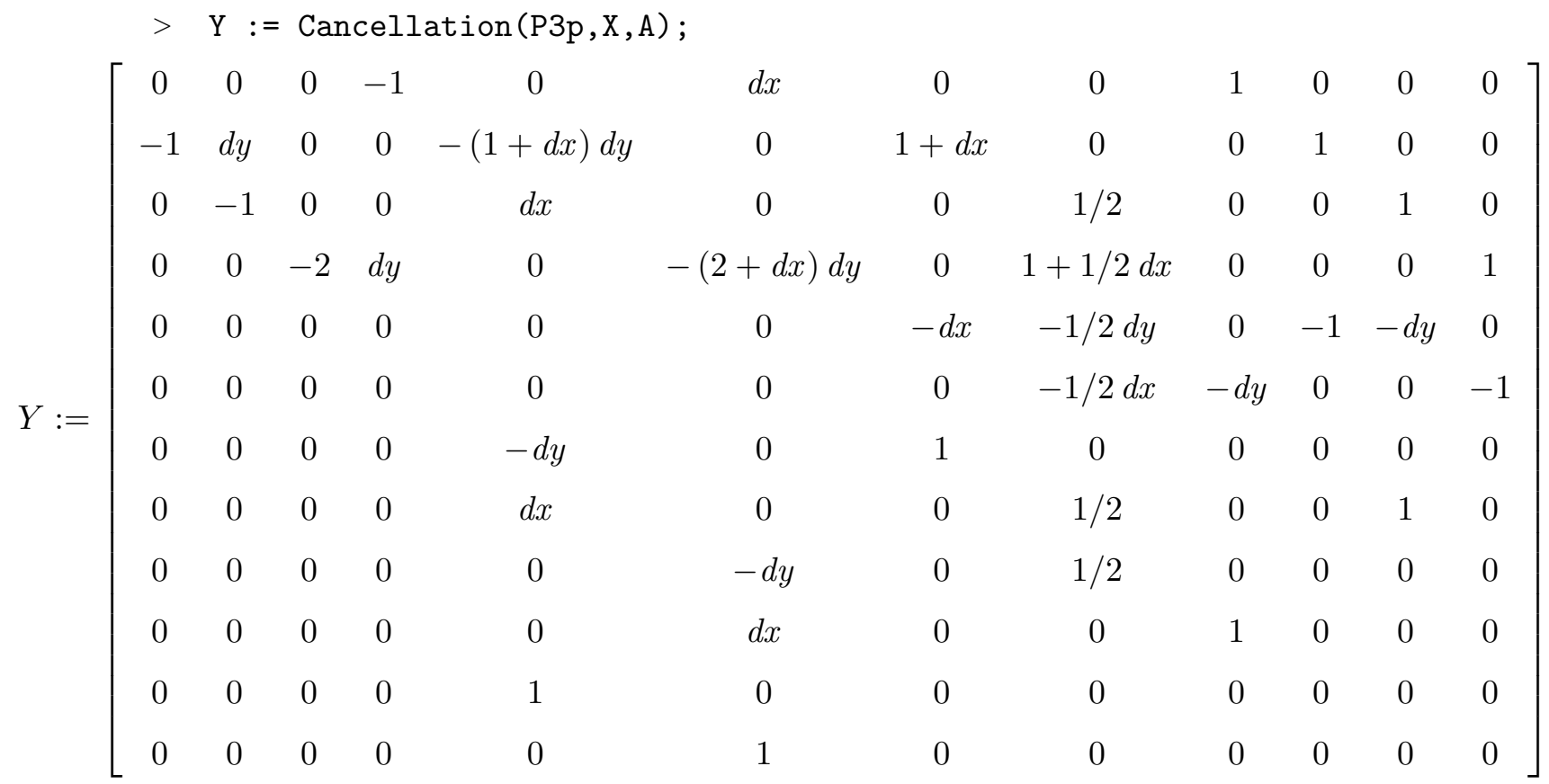

We obtain that $f_{3}: L_{3} \longrightarrow L_{3}^{\prime}$ defined by $f_{3}\left(\tau_{3}(\nu)\right)=\tau_{3}^{\prime}(\nu Y)$ for all $\nu \in A^{1 \times 12}$ is a left $A$ isomorphism, where $\tau_{3}: A^{1 \times 12} \longrightarrow L_{3}$ (resp., $\tau_{3}^{\prime}: A^{1 \times 12} \longrightarrow L_{3}^{\prime}$ ) is the canonical projection onto $L_{3}$ (resp., $L_{3}^{\prime}$ ). We can check again that $f_{3}$ is a left $A$-isomorphism:

$>$ TestIso(P3, P3p, Y, A);

true

$\mathrm{RR} \mathrm{n}^{\circ} 8225$ 
If $P_{3}=\left(\begin{array}{ll}0 & P_{4}\end{array}\right)$, where $P_{4}$ is defined by

$>\mathrm{P} 4:=$ submatrix $(\mathrm{P} 3,1 \ldots 5,2 \ldots 12)$;

$$
P 4:=\left[\begin{array}{ccccccccccc}
0 & 0 & 0 & 1 & 0 & 0 & 0 & 0 & 0 & 0 & 0 \\
0 & 0 & 0 & 0 & 1 & 0 & 0 & 0 & 0 & 0 & 0 \\
0 & 0 & 0 & 0 & 0 & -d x & -d y & 0 & 0 & 0 & 0 \\
0 & 0 & 0 & 0 & 0 & 0 & 1 & -1 & 0 & -d x & -d y \\
0 & 0 & 0 & 0 & 0 & 0 & 0 & -d x & -d y & 0 & 0
\end{array}\right]
$$

then $L_{3} \cong A \oplus L_{4}$, where $L_{4}=A^{1 \times 11} /\left(A^{1 \times 5} P_{4}\right)$. The rank of $L_{4}$ is:

$>\operatorname{OreRank}(\mathrm{P} 4, \mathrm{~A})$;

If $P_{3}^{\prime}=\left(\begin{array}{ll}0 & P_{4}^{\prime}\end{array}\right)$, where $P_{4}^{\prime}$ is defined by

$>\mathrm{P} 4 \mathrm{p}:=$ submatrix $(\mathrm{P} 3 \mathrm{p}, 1 \ldots 5,2 \ldots 12) ;$

$$
P 4 p:=\left[\begin{array}{ccccccccccc}
0 & 0 & 0 & 0 & 0 & -d x & -1 / 2 d y & 0 & 0 & 0 & 0 \\
0 & 0 & 0 & 0 & 0 & 0 & -1 / 2 d x & -d y & 0 & 0 & 0 \\
0 & 0 & 0 & 0 & 0 & 0 & 0 & 0 & 1 & 0 & 0 \\
0 & 0 & 0 & 0 & 0 & 0 & 0 & 0 & 0 & 1 & 0 \\
0 & 0 & 0 & 0 & 0 & 0 & 0 & 0 & 0 & 0 & 1
\end{array}\right]
$$

then $L_{3}^{\prime} \cong A \oplus L_{4}^{\prime}$, where $L_{4}^{\prime}=A^{1 \times 11} /\left(A^{1 \times 5} P_{4}^{\prime}\right)$. The rank of $L_{4}^{\prime}$ is:

$>\operatorname{OreRank}(\mathrm{P} 4 \mathrm{p}, \mathrm{A})$;

Corollary 4 then shows that $L_{4} \cong L_{4}^{\prime}$. Let us compute the corresponding left $A$-isomorphism $f_{4} \in \operatorname{hom}_{A}\left(L_{4}, L_{4}^{\prime}\right)$.

$>\mathrm{Z}:=$ Cancellation $(\mathrm{P} 4 \mathrm{p}, \mathrm{Y}, \mathrm{A})$; 


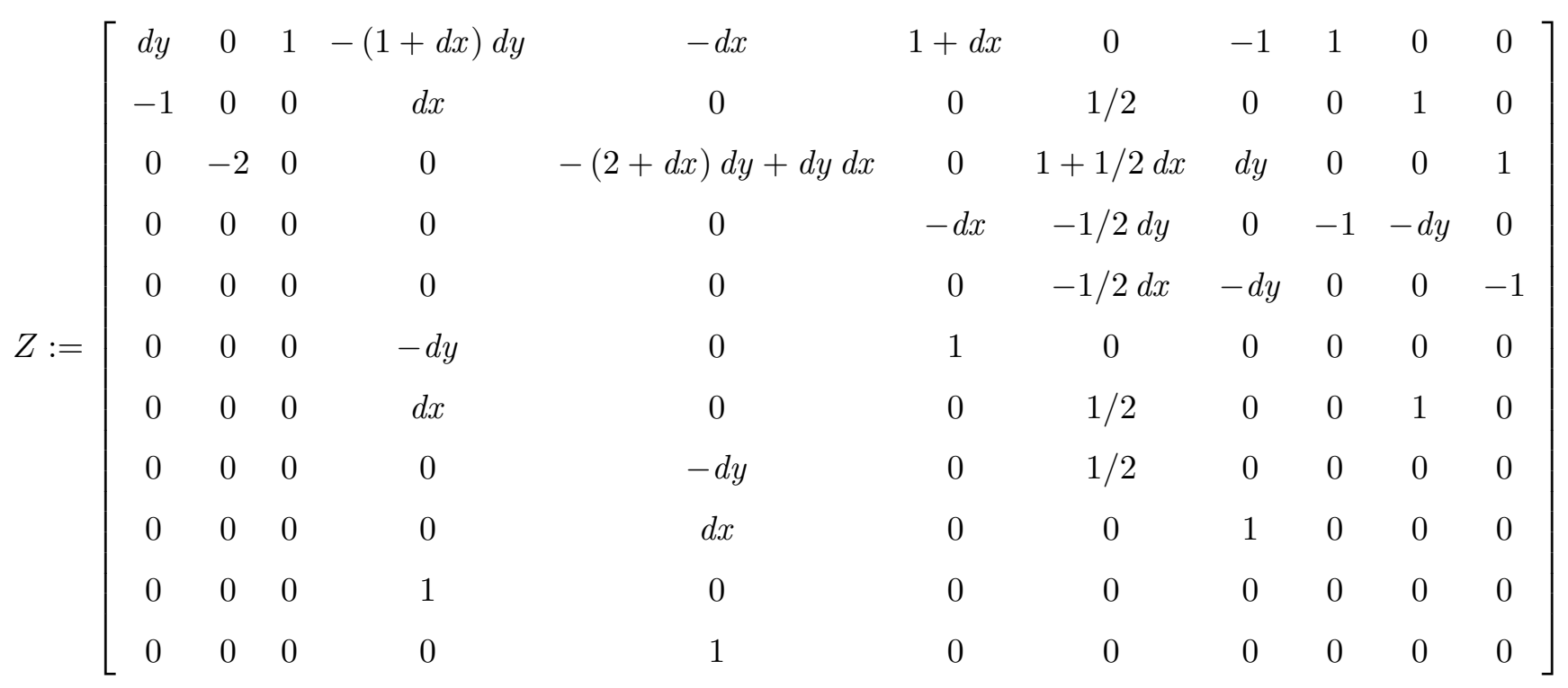

We obtain that $f_{4}: L_{4} \longrightarrow L_{4}^{\prime}$ defined by $f_{4}\left(\tau_{4}(\alpha)\right)=\tau_{4}^{\prime}(\alpha Z)$ for all $\alpha \in A^{1 \times 11}$ is a left $A$ isomorphism, where $\tau_{4}: A^{1 \times 11} \longrightarrow L_{4}$ (resp., $\tau_{4}^{\prime}: A^{1 \times 11} \longrightarrow L_{4}^{\prime}$ ) is the canonical projection onto $L_{4}$ (resp., $L_{4}^{\prime}$ ). We can check again that $f_{4}$ is a left $A$-isomorphism:

$>$ TestIso(P4,P4p,Z,A);

true

If $P_{4}=\left(\begin{array}{ll}0 & P_{5}\end{array}\right)$, where $P_{5}$ is defined by

$>\mathrm{P} 5:=\operatorname{submatrix}(\mathrm{P} 4,1 \ldots 5,2 \ldots 11)$;

$$
P 5:=\left[\begin{array}{cccccccccc}
0 & 0 & 1 & 0 & 0 & 0 & 0 & 0 & 0 & 0 \\
0 & 0 & 0 & 1 & 0 & 0 & 0 & 0 & 0 & 0 \\
0 & 0 & 0 & 0 & -d x & -d y & 0 & 0 & 0 & 0 \\
0 & 0 & 0 & 0 & 0 & 1 & -1 & 0 & -d x & -d y \\
0 & 0 & 0 & 0 & 0 & 0 & -d x & -d y & 0 & 0
\end{array}\right]
$$

then $L_{4} \cong A \oplus L_{5}$, where $L_{5}=A^{1 \times 10} /\left(A^{1 \times 5} P_{5}\right)$. The rank of $L_{5}$ is:

$>\operatorname{OreRank}(\mathrm{P} 5, \mathrm{~A})$;

If $P_{4}^{\prime}=\left(\begin{array}{ll}0 & P_{5}^{\prime}\end{array}\right)$, where $P_{5}^{\prime}$ is defined by

$>$ P5p := submatrix $(\mathrm{P} 4 \mathrm{p}, 1 \ldots 5,2 \ldots 11)$; 


$$
P 5 p:=\left[\begin{array}{cccccccccc}
0 & 0 & 0 & 0 & -d x & -1 / 2 d y & 0 & 0 & 0 & 0 \\
0 & 0 & 0 & 0 & 0 & -1 / 2 d x & -d y & 0 & 0 & 0 \\
0 & 0 & 0 & 0 & 0 & 0 & 0 & 1 & 0 & 0 \\
0 & 0 & 0 & 0 & 0 & 0 & 0 & 0 & 1 & 0 \\
0 & 0 & 0 & 0 & 0 & 0 & 0 & 0 & 0 & 1
\end{array}\right]
$$

then $L_{4}^{\prime} \cong A \oplus L_{5}^{\prime}$, where $L_{5}^{\prime}=A^{1 \times 10} /\left(A^{1 \times 5} P_{5}^{\prime}\right)$. The rank of $L_{5}^{\prime}$ is:

$>\operatorname{OreRank}(\mathrm{P} 5 \mathrm{p}, \mathrm{A})$;

Corollary 4 then shows that $L_{5} \cong L_{5}^{\prime}$. Let us compute the corresponding left $A$-isomorphism $f_{5} \in \operatorname{hom}_{A}\left(L_{5}, L_{5}^{\prime}\right)$.

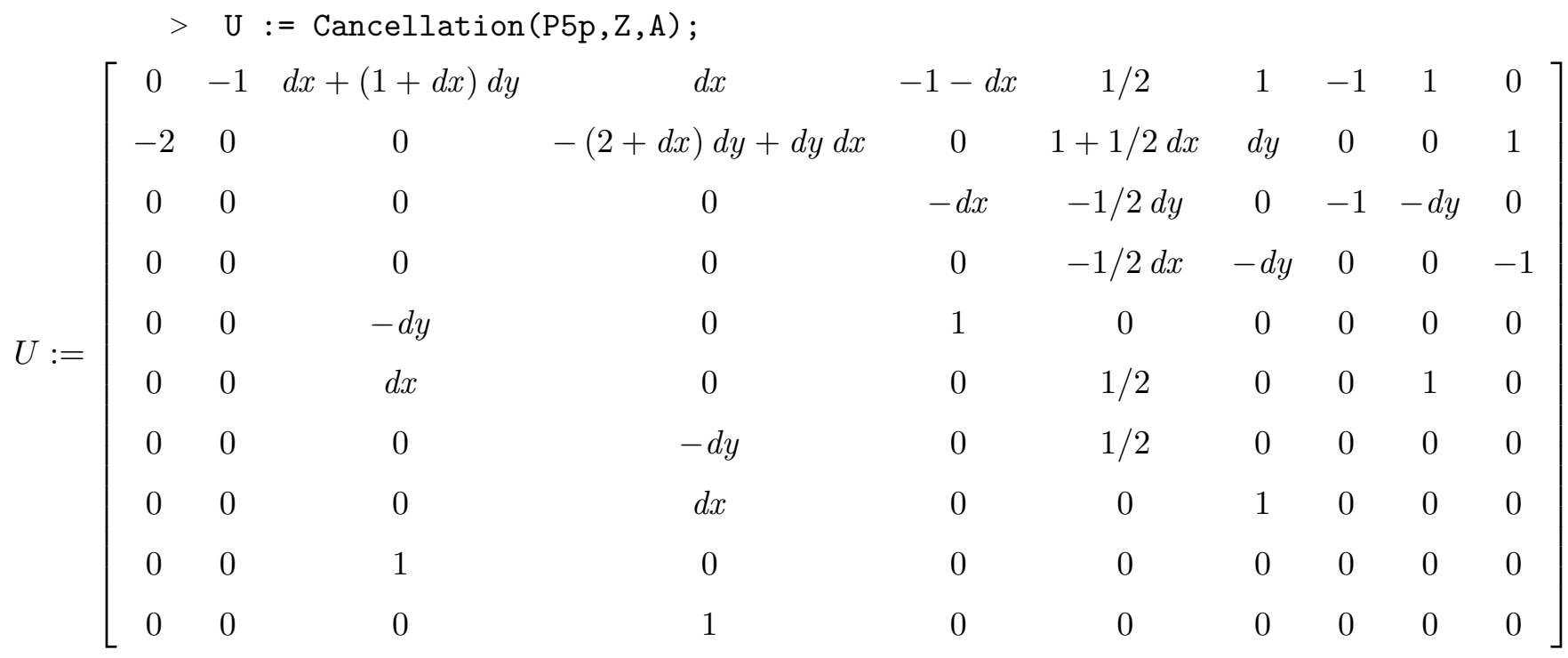

We obtain that $f_{5}: L_{5} \longrightarrow L_{5}^{\prime}$ defined by $f_{5}\left(\tau_{5}(\beta)\right)=\tau_{5}^{\prime}(\beta U)$ for all $\beta \in A^{1 \times 10}$ is a left $A$ isomorphism, where $\tau_{5}: A^{1 \times 10} \longrightarrow L_{5}$ (resp., $\tau_{5}^{\prime}: A^{1 \times 10} \longrightarrow L_{5}^{\prime}$ ) is the canonical projection onto $L_{5}$ (resp., $L_{5}^{\prime}$ ). We can check again that $f_{5}$ is a left $A$-isomorphism:

$>$ TestIso(P5,P5p,U,A);

true

If $P_{5}=\left(\begin{array}{ll}0 & P_{6}\end{array}\right)$, where $P_{6}$ is defined by

$>\mathrm{P} 6:=$ submatrix $(\mathrm{P} 5,1 \ldots 5,2 \ldots 10)$;

$\mathrm{RR} \mathrm{n}^{\circ} 8225$ 


$$
P 6:=\left[\begin{array}{ccccccccc}
0 & 1 & 0 & 0 & 0 & 0 & 0 & 0 & 0 \\
0 & 0 & 1 & 0 & 0 & 0 & 0 & 0 & 0 \\
0 & 0 & 0 & -d x & -d y & 0 & 0 & 0 & 0 \\
0 & 0 & 0 & 0 & 1 & -1 & 0 & -d x & -d y \\
0 & 0 & 0 & 0 & 0 & -d x & -d y & 0 & 0
\end{array}\right]
$$

then $L_{5} \cong A \oplus L_{6}$, where $L_{6}=A^{1 \times 9} /\left(A^{1 \times 5} P_{6}\right)$. The rank of $L_{6}$ is:

$>\operatorname{OreRank}(\mathrm{P} 6, \mathrm{~A})$;

If $P_{5}^{\prime}=\left(\begin{array}{ll}0 & P_{6}^{\prime}\end{array}\right)$, where $P_{6}^{\prime}$ is defined by

$>\quad \mathrm{P} 6 \mathrm{p}:=$ submatrix $(\mathrm{P} 5 \mathrm{p}, 1 . .5,2.10)$;

$$
P 6 p:=\left[\begin{array}{ccccccccc}
0 & 0 & 0 & -d x & -1 / 2 d y & 0 & 0 & 0 & 0 \\
0 & 0 & 0 & 0 & -1 / 2 d x & -d y & 0 & 0 & 0 \\
0 & 0 & 0 & 0 & 0 & 0 & 1 & 0 & 0 \\
0 & 0 & 0 & 0 & 0 & 0 & 0 & 1 & 0 \\
0 & 0 & 0 & 0 & 0 & 0 & 0 & 0 & 1
\end{array}\right]
$$

then $L_{5}^{\prime} \cong A \oplus L_{6}^{\prime}$, where $L_{6}^{\prime}=A^{1 \times 9} /\left(A^{1 \times 5} P_{6}^{\prime}\right)$. The rank of $L_{6}^{\prime}$ is:

$>\operatorname{OreRank}(\mathrm{P} 6 \mathrm{p}, \mathrm{A})$;

Corollary 4 then shows that $L_{6} \cong L_{6}^{\prime}$. Let us compute the corresponding left $A$-isomorphism $f_{6} \in \operatorname{hom}_{A}\left(L_{6}, L_{6}^{\prime}\right)$.

$>\mathrm{V}:=$ Cancellation $(\mathrm{P} 6 \mathrm{p}, \mathrm{U}, \mathrm{A})$; 


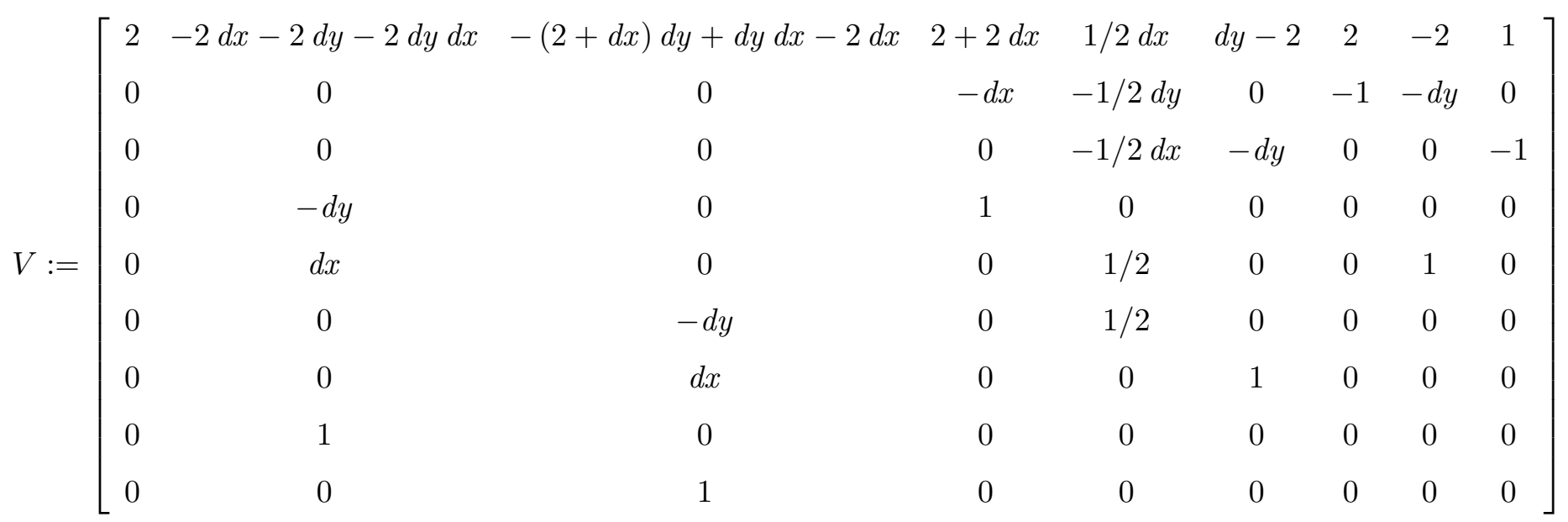

We obtain that $f_{6}: L_{6} \longrightarrow L_{6}^{\prime}$ defined by $f_{6}\left(\tau_{6}(\gamma)\right)=\tau_{6}^{\prime}(\gamma V)$ for all $\gamma \in A^{1 \times 9}$ is a left $A$-isomorphism, where $\tau_{6}: A^{1 \times 9} \longrightarrow L_{6}$ (resp., $\tau_{6}^{\prime}: A^{1 \times 9} \longrightarrow L_{6}^{\prime}$ ) is the canonical projection onto $L_{6}$ (resp., $L_{6}^{\prime}$ ). We can check again that $f_{6}$ is a left $A$-isomorphism:

$>$ TestIso(P6,P6p, V, A);

true

If $P_{6}=\left(\begin{array}{ll}0 & P_{7}\end{array}\right)$, where $P_{7}$ is defined by

$>\mathrm{P} 7:=\operatorname{submatrix}(\mathrm{P} 6,1 \ldots 5,2 \ldots 9)$;

$$
P 7:=\left[\begin{array}{cccccccc}
1 & 0 & 0 & 0 & 0 & 0 & 0 & 0 \\
0 & 1 & 0 & 0 & 0 & 0 & 0 & 0 \\
0 & 0 & -d x & -d y & 0 & 0 & 0 & 0 \\
0 & 0 & 0 & 1 & -1 & 0 & -d x & -d y \\
0 & 0 & 0 & 0 & -d x & -d y & 0 & 0
\end{array}\right]
$$

then $L_{6} \cong A \oplus L_{7}$, where $L_{7}=A^{1 \times 8} /\left(A^{1 \times 5} P_{7}\right)$. The rank of $L_{7}$ is:

$>\operatorname{OreRank}(\mathrm{P} 7, \mathrm{~A})$;

If $P_{6}^{\prime}=\left(\begin{array}{ll}0 & P_{7}^{\prime}\end{array}\right)$, where $P_{7}^{\prime}$ is defined by

$>\mathrm{P} 7 \mathrm{p}:=$ submatrix $(\mathrm{P} 6 \mathrm{p}, 1 . .5,2 . .9)$; 


$$
P 7 p:=\left[\begin{array}{cccccccc}
0 & 0 & -d x & -1 / 2 d y & 0 & 0 & 0 & 0 \\
0 & 0 & 0 & -1 / 2 d x & -d y & 0 & 0 & 0 \\
0 & 0 & 0 & 0 & 0 & 1 & 0 & 0 \\
0 & 0 & 0 & 0 & 0 & 0 & 1 & 0 \\
0 & 0 & 0 & 0 & 0 & 0 & 0 & 1
\end{array}\right]
$$

then $L_{6}^{\prime} \cong A \oplus L_{7}^{\prime}$, where $L_{7}^{\prime}=A^{1 \times 8} /\left(A^{1 \times 5} P_{7}^{\prime}\right)$. The rank of $L_{7}^{\prime}$ is:

$>\operatorname{OreRank}(\mathrm{P} 7 \mathrm{p}, \mathrm{A})$;

Corollary 4 then shows that $L_{7} \cong L_{7}^{\prime}$. Let us compute the corresponding left $A$-isomorphism $f_{7} \in \operatorname{hom}_{A}\left(L_{7}, L_{7}^{\prime}\right)$.

$>\mathrm{W}:=\operatorname{map}(\operatorname{collect}$, Cancellation $(\mathrm{P} 7 \mathrm{p}, \mathrm{V}, \mathrm{A}),[\mathrm{y}, \mathrm{dx}, \mathrm{dy}])$ :

We obtain that $f_{7}: L_{7} \longrightarrow L_{7}^{\prime}$ defined by $f_{7}\left(\tau_{7}(\theta)\right)=\tau_{7}^{\prime}(\theta W)$ for all $\theta \in A^{1 \times 8}$ is a left $A$-isomorphism, where $\tau_{7}: A^{1 \times 8} \longrightarrow L_{7}$ (resp., $\tau_{7}^{\prime}: A^{1 \times 8} \longrightarrow L_{7}^{\prime}$ ) is the canonical projection onto $L_{7}$ (resp., $L_{7}^{\prime}$ ). We can check again that $f_{7}$ is a left $A$-isomorphism:

$>$ TestIso(P7,P7p,W,A);

true

Let us compute a matrix $Q$ satisfying $P_{7} W=Q P_{7}^{\prime}$ :

$>\mathrm{Q}:=$ Factorize $(\operatorname{Mult}(\mathrm{P} 7, \mathrm{~W}, \mathrm{~A}), \mathrm{P} 7 \mathrm{p}, \mathrm{A})$;

$$
Q:=\left[\begin{array}{ccccc}
1 & 0 & -1 & -d y & 0 \\
0 & 1 & 0 & 0 & -1 \\
1 & 0 & 0 & -d y & 0 \\
0 & 0 & 0 & 1 & 0 \\
0 & 1 & 0 & 0 & 0
\end{array}\right]
$$

If $P_{7}=\left(\begin{array}{ll}0 & P_{8}\end{array}\right)$, where $P_{8}$ is defined by

$>\mathrm{P} 8:=\operatorname{submatrix}(\mathrm{P} 7,3 . .5,3.8)$;

$$
P 8:=\left[\begin{array}{cccccc}
-d x & -d y & 0 & 0 & 0 & 0 \\
0 & 1 & -1 & 0 & -d x & -d y \\
0 & 0 & -d x & -d y & 0 & 0
\end{array}\right]
$$

then $L_{7} \cong A \oplus L_{8}$, where $L_{8}=A^{1 \times 7} /\left(A^{1 \times 5} P_{8}\right)$. The rank of $L_{8}$ is: 
$>\operatorname{OreRank}(\mathrm{P} 8, \mathrm{~A})$;

If $P_{7}^{\prime}=\left(\begin{array}{ll}0 & P_{8}^{\prime}\end{array}\right)$, where $P_{8}^{\prime}$ is defined by

$>$ P8p := submatrix $(\mathrm{P} 7 \mathrm{p}, 1 \ldots 2,1 \ldots 5)$;

$$
P 8 p:=\left[\begin{array}{ccccc}
0 & 0 & -d x & -1 / 2 d y & 0 \\
0 & 0 & 0 & -1 / 2 d x & -d y
\end{array}\right]
$$

then $L_{7}^{\prime} \cong A \oplus L_{8}^{\prime}$, where $L_{8}^{\prime}=A^{1 \times 7} /\left(A^{1 \times 5} P_{8}^{\prime}\right)$. The rank of $L_{8}^{\prime}$ is:

$>\operatorname{OreRank}(\mathrm{P} 8 \mathrm{p}, \mathrm{A})$;

Let us consider the matrix $W^{\prime}$ obtained by taking the last five rows of $W$

$>\operatorname{Wp}:=\operatorname{submatrix}(\mathrm{W}, 3 . .8,1 \ldots 5)$ :

i.e., the rows of $W^{\prime}$ are respectively defined by:

$>$ for $i$ from 1 to 5 do submatrix(Wp,1..2, i..i); od;

$\left[\begin{array}{c}\left(-6 d y^{2}-3 d y^{3}-3 d y\right) d x^{2}+\left(3-3 d y^{4}+(-9+3 y) d y^{3}+(6 y-6) d y^{2}+(3+3 y) d y\right) d x-3 d y^{4}+(-3+3 y) d y^{3}+3 y d y^{2}+2 d y \\ \left(6 d y+3 d y^{2}+3\right) d x^{3}+\left(3 d y^{3}+(9-3 y) d y^{2}+(9-6 y) d y+3-3 y\right) d x^{2}+\left(1+3 d y^{3}+(3-3 y) d y^{2}+(3-3 y) d y\right) d x\end{array}\right]$ $\left[\begin{array}{c}\left(-3 d y^{2}-3 d y\right) d x^{2}+\left(6-6 d y^{3}+(6 y-6) d y^{2}+(6 y+6) d y\right) d x-3 d y^{4}+(-3+6 y) d y^{3}+\left(9+6 y-3 y^{2}\right) d y^{2}+\left(9-6 y-3 y^{2}\right) d y-6 y \\ (3 d y+3) d x^{3}+\left(6 d y^{2}+(-6 y+6) d y-6 y\right) d x^{2}+\left(3 d y^{3}+(3-6 y) d y^{2}+\left(-6 y+3 y^{2}-3\right) d y-3+3 y^{2}\right) d x\end{array}\right]$

$$
\begin{gathered}
{\left[\begin{array}{c}
\left(3 d y^{2}+3 d y\right) d x^{2}+\left(-3+3 d y^{3}+(6-3 y) d y^{2}+(3-3 y) d y\right) d x-2+3 d y^{3}+(3-3 y) d y^{2}-3 y d y \\
(-3 d y-3) d x^{3}+\left(-3 d y^{2}+(-6+3 y) d y-6+3 y\right) d x^{2}+\left(-3 d y^{2}+(-3+3 y) d y-3+3 y\right) d x
\end{array}\right]} \\
{\left[\begin{array}{c}
\left(3 / 4 d y^{2}+3 / 4 d y\right) d x^{2}+\left(-3 / 4+3 / 4 d y^{3}+(-3 / 4 y+3 / 4) d y^{2}-3 / 4 y d y\right) d x \\
1 / 2+(-3 / 4 d y-3 / 4) d x^{3}+\left(-3 / 4 d y^{2}+(-3 / 4+3 / 4 y) d y-3 / 4+3 / 4 y\right) d x^{2}
\end{array}\right]} \\
{\left[\begin{array}{c}
\left(3 / 2 d y^{3}-3 / 2 d y^{2}-3 d y\right) d x+3+3 / 2 d y^{4}+(-3 / 2-3 / 2 y) d y^{3}+(3 / 2 y-3) d y^{2}+(-3 / 2+3 y) d y \\
\left(3-3 / 2 d y^{2}+3 / 2 d y\right) d x^{2}+\left(-3 / 2 d y^{3}+(3 / 2+3 / 2 y) d y^{2}+(3 / 2-3 / 2 y) d y+3-3 y\right) d x
\end{array}\right]}
\end{gathered}
$$

Let us consider the matrix $Q^{\prime}$ obtained by taking the last three rows of $Q$ :

$>\mathrm{Qp}:=\operatorname{submatrix}(\mathrm{Q}, 3 \ldots 5,1 \ldots 2)$;

$$
Q p:=\left[\begin{array}{ll}
1 & 0 \\
0 & 0 \\
0 & 1
\end{array}\right]
$$

We can easily check that $P_{8} W^{\prime}=Q^{\prime} P_{8}^{\prime}$ :

$\mathrm{RR} \mathrm{n}^{\circ} 8225$ 
$>\operatorname{simplify}(\operatorname{evalm}(\operatorname{Mult}(\mathrm{P} 8, W p, A)-\operatorname{Mult}(\mathrm{Qp}, \mathrm{P} 8 \mathrm{p}, \mathrm{A})))$;
\[ \left[\begin{array}{ccccc}0 & 0 & 0 & 0 & 0 \\ 0 & 0 & 0 & 0 & 0 \\ 0 & 0 & 0 & 0 & 0\end{array}\right] \]

We can also check that $f_{8}: L_{8} \longrightarrow L_{8}^{\prime}$ defined by $f_{8}\left(\tau_{8}(v)\right)=\tau_{8}^{\prime}\left(v W^{\prime}\right)$ for all $v \in A^{1 \times 7}$ is a left $A$-isomorphism, where $\tau_{8}: A^{1 \times 7} \longrightarrow L_{8}$ (resp., $\tau_{8}^{\prime}: A^{1 \times 7} \longrightarrow L_{8}^{\prime}$ ) is the canonical projection onto $L_{8}$ (resp., $\left.L_{8}^{\prime}\right)$ :

$>$ TestIso(P8,P8p,Wp,A);

true

We note that the left $A$-module $L_{8}$ corresponds to Cosserat's equations (see Examples 6 and 21). Moreover, from the structure of the matrix $P_{8}^{\prime}$, it is clear that $L_{8}^{\prime} \cong A^{1 \times 2} \oplus L_{9}^{\prime}$, where $L_{9}^{\prime}=A^{1 \times 3} /\left(A^{1 \times 2} P_{9}^{\prime}\right)$ and $P_{9}^{\prime}$ is defined by:

$>\mathrm{P} 9 \mathrm{p}:=$ submatrix $(\mathrm{P} 8 \mathrm{p}, 1 \ldots 2,3 . .5)$;

$$
P 9 p:=\left[\begin{array}{ccc}
-d x & -1 / 2 d y & 0 \\
0 & -1 / 2 d x & -d y
\end{array}\right]
$$

We also note that $L_{9}^{\prime}$ corresponds to the linear PD system defined by the equilibrium of the symmetric stress tensor (see Examples 6 and 21). Hence, we find again that $L_{8} \cong A^{1 \times 2} \oplus L_{9}^{\prime}$.

\section{References}

[1] M. Barakat, D. Robertz, "homalg: A meta-package for homological algebra", J. Algebra Appl., 7 (2008), 299-317. 6

[2] M. Barakat, M. Lange-Hegermann, "An axiomatic setup for algorithmic homological algebra and an alternative approach to localization", J. Algebra Appl., 10 (2011), 269293. 6

[3] M. Barakat, B. Bremer, "Higher extension modules and the Yoneda product", preprint arXiv:math.KT/0802.3179 (http://arxiv.org/abs/0802.3179), submitted for publication, 2008. 6

[4] M. Barakat, "Spectral filtrations via generalized morphisms", preprint arXiv:0904.0240 (http://arxiv.org/abs/0904.0240), submitted for publication, 2009. 6

[5] H. Bass, Algebraic K-Theory, Benjamin, 1968. 6

[6] J. E. Björk, Rings of Differential Operators, North Holland, 1979. 7, 8, 16, 44

$\mathrm{RR} \mathrm{n}^{\circ} 8225$ 
[7] M. S. Boudellioua, A. Quadrat, "Serre's reduction of linear functional systems", Math. Comput. Sci., 4 (2010), 289-312. 6, 7, 40, 44

[8] L. H. Byun, "A note on the module structure of Weyl algebras and simple noetherian rings", Comm. Algebra, 21 (1993), 991-998. 61

[9] N. Caro, D. Levcovitz, "On a Theorem of Stafford", Cadernos de Mathemática, 11 (2010), 63-70. 5, 6, 17

[10] R. C. Churchill, J. J. Kovacic, "Cyclic vectors", Proceedings of Algebra and Related Topics, Rutgers University, Newark, November 2-3, 2002. 7, 42

[11] F. Chyzak, A. Quadrat, D. Robertz, "Effective algorithms for parametrizing linear control systems over Ore algebras", Appl. Algebra Engrg. Comm. Comput., 16 (2005), 319 376. $6,7,8,9,10,12,13,14,19,24,27,39,43,46,54,56,81,97$

[12] F. Chyzak, A. Quadrat, D. Robertz, "OreModules: A symbolic package for the study of multidimensional linear systems", in Applications of Time-Delay Systems, J. Chiasson and J.-J. Loiseau (eds.), Lecture Notes in Control and Inform. 352, Springer, 2007, 233264, OreModules project: http://wwwb.math.rwth-aachen.de/OreModules. 6, 13, 14,61

[13] T. Cluzeau, A. Quadrat, "Factoring and decomposing a class of linear functional systems", Linear Algebra Appl., 428 (2008), 324-381. 5, 6, 7, 8, 9, 11, 18, 27

[14] T. Cluzeau, A. Quadrat, "OreMorphisms: A homological algebraic package for factoring and decomposing linear functional systems", in Topics in Time-Delay Systems: Analysis, Algorithms and Control, J.-J. Loiseau, W. Michiels, S.-I. Niculescu and R. Sipahi (eds.), Lecture Notes in Control and Inform. 388, Springer, 2009, 179-196, OrEMORPHISMS project: http://pages.saclay.inria.fr/alban.quadrat/ /OreMorphisms/index.html. 6, 58, 61, 69, 70

[15] T. Cluzeau, A. Quadrat, "Serre's reduction of linear partial differential systems with holonomic adjoints", J. Symbolic Comput., 47 (2012), 1192-1213. 5, 6, 7, 40, 44

[16] T. Cluzeau, A. Quadrat, "A constructive version of Fitting's theorem on isomorphisms and equivalences of linear systems", Proceedings of $n D S^{\prime} 11$, Poitiers, France, 2011. 6, 33, $56,57,58$

[17] T. Coquand, H. Lombardi, C. Quitté, "Generating non-Noetherian modules constructively", Manuscripta Math., 115 (2004), 513-520. 6

[18] S. C. Coutinho, M. P. Holland, "Module structure of rings of differential operators", Proc. London Math. Soc., 57 (1988), 417-432. 5, 6, 14, 15, 16, 17, 61

$\mathrm{RR} \mathrm{n}^{\circ} 8225$ 
[19] D. Eisenbud, Commutative Algebra with a View Toward Algebraic Geometry, Graduate Texts in Mathematics 150, Springer, 1995. 46

[20] J. Gago-Vargas, "Bases for projective modules in $A_{n}(k)$ ", J. Symbolic Comput., 36 (2003), 845-853. 5, 11, 53, 60

[21] A. Hillebrand, W. Schmale, "Towards an effective version of a theorem of Stafford", J. Symbolic Comput., 32 (2001), 699-716. 5, 16, 60

[22] R. E. Kalman, P. L. Falb, M. A. Arbib, Topics in Mathematical System Theory, McGrawHill, 1969. 40, 41

[23] M. Kashiwara, Algebraic Study of Systems of Partial Differential Equations, Master Thesis, Tokyo Univ., 1970, Mémoires de la Société Mathématiques de France 63 (1995) (English translation). 7, 8

[24] T. Y. Lam, Lectures on Modules and Rings, Graduate Texts in Mathematics 189, Springer, 1999. 12, 15, 53

[25] A. Leykin, "Algorithmic proofs of two theorems of Stafford", J. Symbolic Comput., 38 (2004), 1535-1550. 5, 16, 60

[26] A. Leykin, H. Tsai, "Dmodules - Algorithms for D-modules", Macaulay2 package, http: //www.math.uiuc.edu/Macaulay2/. 5

[27] H. Lombardi, C. Quitté, Algèbre commutative, Calvage \& Mounet, 2011. 6

[28] P. Maisonobe, C. Sabbah, D-modules cohérents and holonomes, Hermann, 1993. 7, 8, 16,44

[29] B. Malgrange, "Systèmes différentiels à coefficients constants", Séminaire Bourbaki 1962/63, 1-11. 7, 8

[30] J. C. McConnell, J. C. Robson, Noncommutative Noetherian Rings, American Mathematical Society, 2000. 10, 15, 16, 17, 18, 42

[31] J.-F. Pommaret, Partial Differential Control Theory, Mathematics and Its Applications, Kluwer Academic Publishers, 2001. 6, 13, 57

[32] J.-F. Pommaret, A. Quadrat, "Algebraic analysis of linear multidimensional control systems", IMA J. Math. Control Inform., 16 (1999), 275-297. 6

[33] A. Quadrat, "An introduction to constructive algebraic analysis and its applications", Les cours du CIRM, 1 no. 2: Journées Nationales de Calcul Formel (2010), 281-471 (http://hal.archives-ouvertes.fr/inria-00506104/fr/). 6, 9, 10, 14, 42

$\mathrm{RR} \mathrm{n}^{\circ} 8225$ 
[34] A. Quadrat, "Grade filtration of linear functional systems", to appear in Acta Appl. Math., 2013, DOI: 10.1007/s10440-012-9791-2. 6, 9, 56

[35] A. Quadrat, D. Robertz, "On the blowing-up of stably free behaviours", Proceedings of CDC-ECC05, Seville, Spain, 2005. 54

[36] A. Quadrat, D. Robertz, "Computation of bases of free modules over the Weyl algebras", J. Symbolic Comput., 42 (2007), 1113-1141, STAFFord project (http://wwwb.math. rwth-aachen.de/OreModules). 5, 6, 7, 8, 9, 10, 11, 14, 16, 17, 42, 44, 46, 49, 54, 60, 97, 98

[37] A. Quadrat, D. Robertz, "Controllability and differential flatness of linear analytic ordinary differential systems", Proceedings of MTNS 2010, Budapest, Hungary, 2010. Also in: E. Zerz (ed.), Algebraic Systems Theory, Behaviors, and Codes, Shaker, 2010, 23-30. 5,17

[38] J. J. Rotman, An Introduction to Homological Algebra, Springer, $2^{\text {nd }}$ edition, 2009. 9, $10,12,15,16,18,19,27,33,43,45,54$

[39] J.-P. Serre, "Modules projectifs et espaces fibrés à fibre vectorielle", Séminaire P. Dubreil, année 1957/1958. 6, 17

[40] J. T. Stafford, "Stable structure of noncommutative noetherian rings", J. Algebra, 47 (1977), 244-267. 6

[41] J. T. Stafford, "Stable structure of noncommutative noetherian rings, II", J. Algebra, 52 (1978), 218-235. 6

[42] J. T. Stafford, "Module structure of Weyl algebras", J. London Math. Soc., 18 (1978), 429-442. 5, 6, 10, 16, 17, 18, 27, 34, 44, 46, 47, 50, 51, 60, 61

[43] R. G. Swan, Algebraic K-theory, Lecture Notes in Mathematics 76, Springer, 1968. 46, 50

$\mathrm{RR} \mathrm{n}^{\circ} 8225$ 


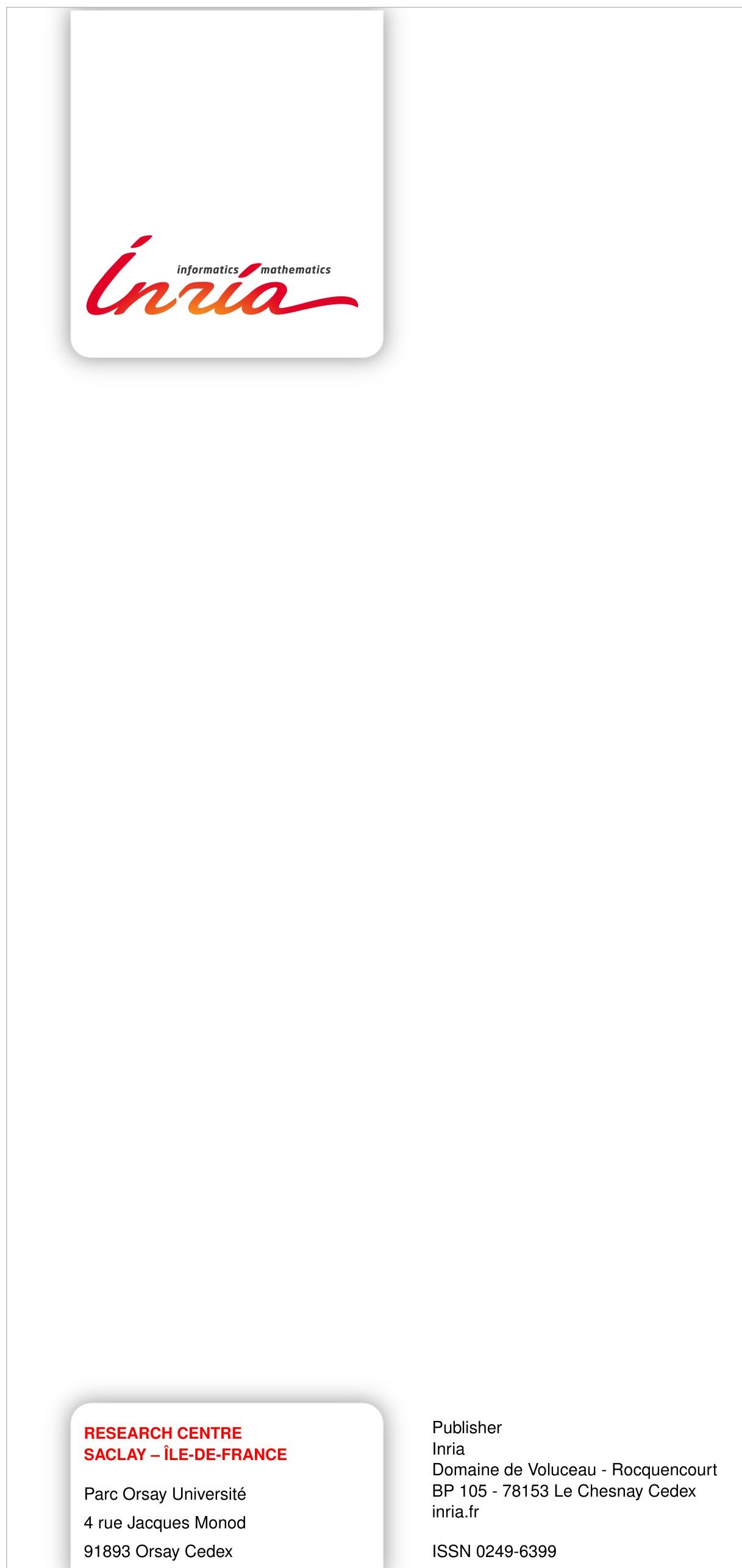

